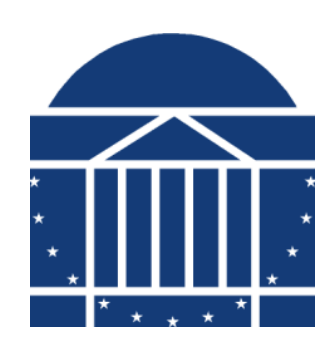

\title{
Simulation of Spray-Enhanced Compressed Air Energy Storage for Wind Turbines
}

\begin{abstract}
A Dissertation Presented to the Faculty of the School of Engineering and Applied Science University of Virginia
\end{abstract}

\author{
In Partial Fulfillment \\ of the Requirements for the Degree of \\ Doctor of Philosophy in Mechanical and Aerospace Engineering
}

by

\section{CHAO QIN}

B.S., Tsinghua University, Beijing, China - 2008

August 2014 
Approval Sheet

The dissertation

is submitted in partial fulfillment of the requirements

for the degree of

Doctor of Philosophy

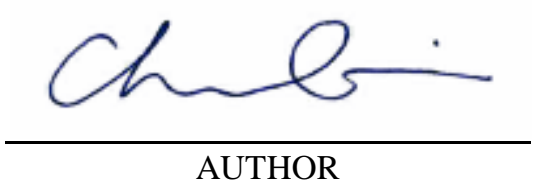

This dissertation has been read and approved by the examining committee:

\author{
Prof. Eric Loth \\ Advisor / Dissertation director

\section{Prof. Harsha Chelliah} \\ Committee member \\ $\underline{\text { Prof. Joanna Curran }}$ \\ Outside committee member \\ Prof. Haibo Dong \\ Committee chairperson

\section{$\underline{\text { Prof. Robert Ribando }}$}

Committee member

Accepted for the School of Engineering and Applied Science:

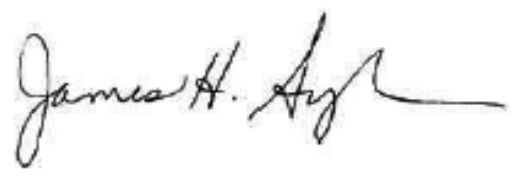

Dean, School of Engineering and Applied Science 


\section{Abstract}

Growing size of wind turbines, especially off-shore wind turbines, has strong advantages of increased wind speed with less turbulent at higher altitudes and the larger rotor sizes that can capture more energy. A hybrid hydraulic-electric wind turbine concept is proposed by introducing a hydraulic motor in the nacelle to convert rotor shaft work into hydraulic power that can be transmitted to the electric generator at ground/sea level. This hybrid design can greatly reduce the nacelle/drive components, help simplify or even eliminate the gearbox to reduce maintenance, and greatly reduce the head weight as much as $50 \%$. To quantify the benefits of a hybrid hydraulic-electric power generation and of possible energy storage, the National Renewable Energy Laboratory (NREL) off-shore 5MW baseline wind turbine was employed as a baseline. Based on analysis, the tower mass can be reduced by $33 \%$ to $50 \%$, maintaining the tower natural frequencies. So the capital costs of wind turbine manufacture and assembly can be reduced.

Integrating Compressed Air Energy Storage (CAES) to a power supply system with a variable and unsteady energy source can also bring a number of advantages. The hydraulic-electric generator concept is compatible with a novel approach to compressed air energy storage that allows high storage efficiency. This concept involves a liquid piston utilized as a compressor and expander with water spray to promote heat transfer to allow near isothermal processes. For the 5MW reference off-shore wind turbine which integrates a spray-based compressed air energy storage with a $35 \mathrm{MPa}$ accumulator, the overall compression is proposed in three stages with pressure ratios of 10:1, 7:1, and 5:1, all operated at $1 \mathrm{~Hz}$ to maintain moderate liquid surface acceleration. Based on a simple 
and fundamental description of the system, compression efficiency is found to be strongly dependent on droplet surface area, which can be achieved through either high mass loading or small drop sizes. The one-dimensional simulations show that direct injection spray can increase overall three-stage compression efficiency to as high as $89 \%$, substantially better than the $27 \%$ associated with a conventional adiabatic compression at the same pressure ratio. In addition, this liquid piston concept is examined with detailed two-dimensional flow. Multi-phase computational fluid dynamics is implemented in an axisymmetric domain to investigate compression in a cylinder for first-stage and secondstage compression, using a spray discharge within the cylinder at various mass loadings. The spray was generally achieved by operating a single pressure-swirl nozzle directed along the centerline and operating at the maximum liquid mass flux capable that still permitted a small average droplet diameter. The results indicating that a single pressureswirl nozzle injection resulted in an injected mass loading of 1.6 and yielded efficiency as high as $93 \%$ for a first-stage compression cycle. To satisfy both high mass loading and uniform distribution for second-stage operation, a multi-nozzle configuration should be considered. Pre-mixed approach may serve to increase the degree of mixing and the mass of drops than can be injected. 


\section{Acknowledgments}

I would never be able to finishing this dissertation without the meticulous guidance of my committee members, generous help from my colleagues and friends, as well as solid support from my family and girlfriend.

I would like to express my deepest gratitude to my dissertation advisor Dr. Eric Loth, who has been a nice mentor. His excellent guidance, practical advice, and enduring encouragement have been an inexhaustible source of support for me during my research.

I would like to thank Dr. Harsha Chelliah, Dr. Joanna Curran, Dr. Haibo Dong, and Dr. Robert Ribando for their advice and feedback that helped me to strengthen my work.

I would also like to thank Dr. Perry Li, Dr. Terrence Simon, and Dr. James Van de Ven, who are from University of Minnesota, for helping me to develop my background of the compressed air energy storage system.

Thank the National Science Foundation (NSF) and Dominion Virginia Power, based in Richmond, Virginia, to support my research.

最后, 感谢我的父母及家人。在离家近十年的求学过程中, 谢谢他们对我的关心、 支持和理解。他们也是我完成博士论文的动力。 


\section{Contents}

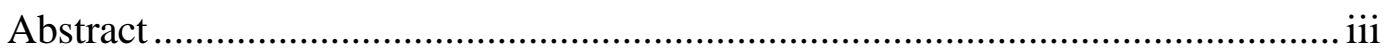

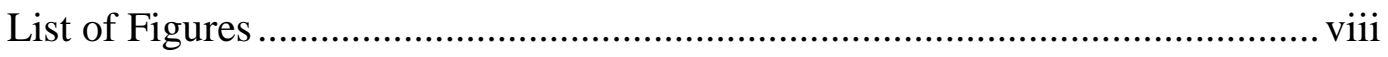

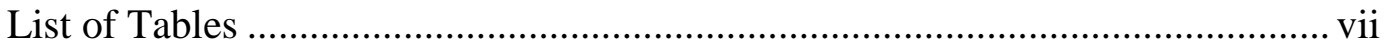

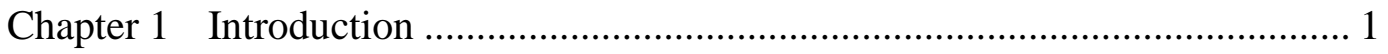

Chapter 2 Liquid Piston Compression with Droplet Heat Transfer ..................... 7

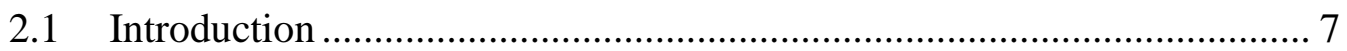

2.2 Overall Concept and Spray Injection Options.................................. 11

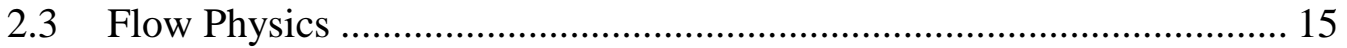

2.4 Numerical Treatment.................................................................... 22

2.5 Test Conditions for Off-Shore Wind Turbines ................................. 25

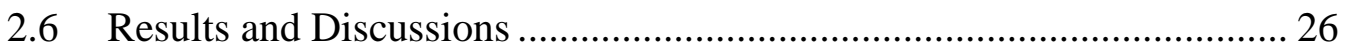

Appendix A. Validation of Spray Cooling .................................................... 36

Appendix B. Thermodynamic and Psychrometric Properties of Humid Air.... 40

Chapter 3 Spray-Cooling Concept for Wind-Based CAES ............................ 46

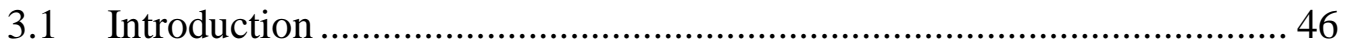

3.2 Storage Concept, Design, and Analysis ........................................ 48

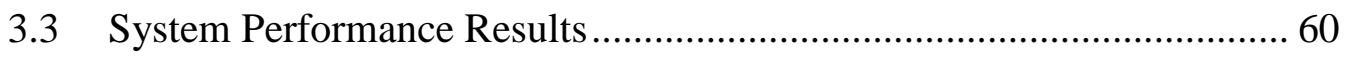

Chapter 4 Numerical Description of a Pressure-Swirl Nozzle Spray ............... 76 


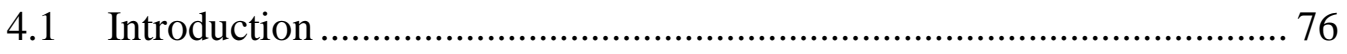

4.2 Flow Physics and Numerical Methodology ….................................... 78

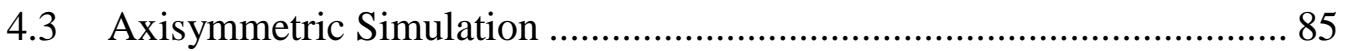

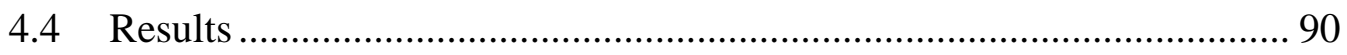

Chapter 5 Potential Benefits of Hydraulic-Electric Generators ..................... 100

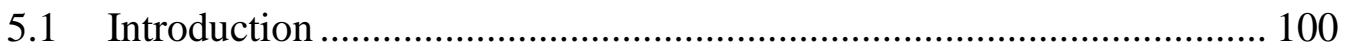

5.2 Tower Structural Model ........................................................... 103

5.3 Impact of Hydraulics on Head and Tower Mass.............................. 107

5.4 Impact of Tower Design on Tower Storage ................................... 111

Chapter 6 Simulation of Spray Direct Injection Compression ....................... 118

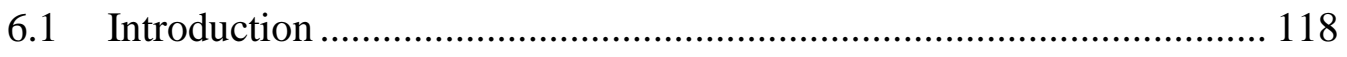

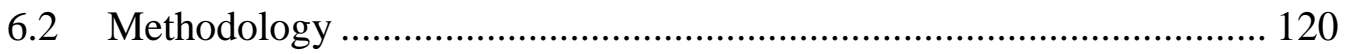

6.3 Test Conditions and Computational Domain ................................. 128

6.4 Results and Discussions ........................................................ 132

Chapter 7 Simulation of Spray in a Premixed Feeder Tube .......................... 147

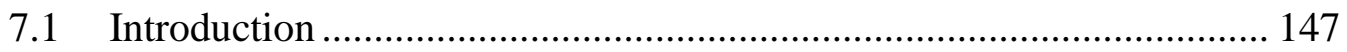

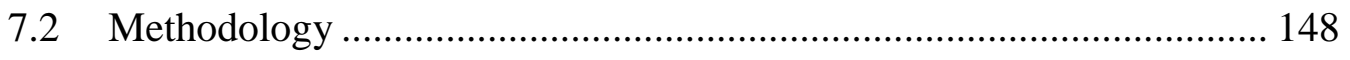

7.3 Feeder Tube Test Conditions ..................................................... 154

7.4 Results and Discussions ........................................................... 157

Chapter 8 Summary and Recommendations .......................................... 164 


\section{List of Figures}

Fig. 2.1 Concept for direct injection of water droplets with a liquid piston .................... 13 Fig. 2.2 Concept for pre-mixed injection of water droplets in an upstream passage........ 14 Fig. 2.3 Vertical coordinate system for positions of droplets and liquid piston interface 16 Fig. 2.4 Temperature, Pressure, and Compression Work vs. Piston Travel for Pre-mixed Injection $(\mathrm{d}=20 \mu \mathrm{m}, \mathrm{CR}=10)$. 30

Fig. 2.5 Temperature, Pressure, and Compression Work vs. Piston Travel for Direct Injection $(\mathrm{d}=20 \mu \mathrm{m}, \mathrm{CR}=10)$.

Fig. 2.6 Temperature vs. Piston Travel for $\mathrm{CR}=10, \mathrm{ML}=1$, with droplet diameters of 10 ,

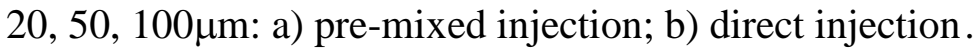
32

Fig. 2.7 Normalized Droplet Surface Area vs. Piston Travel $(C R=10, d=20 \mu m, M L=1) 34$

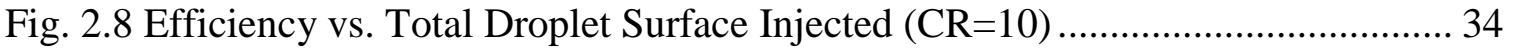

Fig. 2.9 Efficiency a) $\mathrm{CR}=10$ and variable $\mathrm{ML}$; b) $\mathrm{ML}=1$ and variable $\mathrm{CR}(\mathrm{d}=20 \mu \mathrm{m}) \ldots 35$

Fig. 3.1 Sketch of conventional offshore wind energy transmission ............................. 46

Fig. 3.2 Schematic indicating use of energy storage that provides a constant electrical generator output despite unsteady available wind power .... 48

Fig. 3.3 Open accumulator storage volume for one, two and four days worth of energy storage (solid lines) compared the tower inner volume (dashed line) whereby tower height and diameter are assumed to scale linearly with rotor diameter, which is assumed to be proportional to the square root of rated power.

Fig. 3.4 Open accumulator concept operation when: a) energy is both transmitted and stored due to high wind power availability, and b) energy is regenerated due to no wind power availability

Fig. 3.5 Air pressure as a function of its specific volume comparing an isothermal compression and expansion process (both shown by blue line) and an adiabatic compression and expansion process coupled with non-adiabatic heat rejection to atmosphere while in storage (shown by red line circuit whereby net integral results in energy loss)

Fig. 3.6 Arrangement of three-stage air compression for offshore wind energy storage assuming constant volume and frequency for the all compression cylinders .................. 53 Figure 3.7 Concept of direction injection as a function of stroke angle

Fig. 3.8 Compression characteristics as a function of piston travel for all three stages for a fixed final compression ratio: a) compression work; and b) temperature increase .......... 62 Fig. 3.9 Experimental performance of various single nozzles in terms of liquid flow rate and SMD, where air injection nozzles are denoted with circle symbols (from data available in Ref. 16 and 17), whereas liquid-flow only nozzles are denoted with triangular symbols (from data available in Ref. 15)....

Fig. 3.10 Gas liquid ratio (GLR) impact on liquid piston efficiency for various stages for a spray droplet size of $30 \mu \mathrm{m}$ and a spray mass loading of unity 66 Fig. 3.11 Compressive efficiency as function of liquid flow rate and spray drop size for first stage and a reasonably achievable nozzle design point that results in $95 \%$ efficiency

Fig. 4.1 Comparison of numerical results to the experiment of Snyder and Lumley ${ }^{[19]}$ for

a) turbulent kinetic energy and b) dissipation rate 82 
Fig. 4.2 Numerical results of dispersion of four kinds of particles (with increasing particle response time): a) hollow glass $(0.00174 \mathrm{sec})$, b) corn pollen $(0.0223 \mathrm{sec})$, c) solid glass

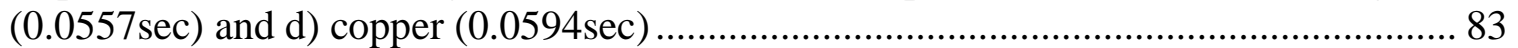

Fig. 4.3 Diffusion comparison of numerical results to the experiment of Snyder and Lumley ${ }^{[19]}$

Fig. 4.4 Sketch of simulation domain based on figure from Santolaya et al. ${ }^{[8]}$ 84

Fig 4.5 Axial velocity input at $x=9 \mathrm{~mm}$ : a) mean and fluctuating velocities for large particles 88

Fig. 4.6 Definition of droplet mass flux inflow boundary conditions as a function of drop size .

Fig. 4.7 Simulation result (left: without particle; right: with particles): a) contours of gas velocity magnitude $(\mathrm{m} / \mathrm{s})$, and b) contours of turbulent kinetic energy $\left(\mathrm{m}^{2} / \mathrm{s}^{2}\right)$....

Fig. 4.8 Comparison between CFD and experiment at $\mathrm{x}=18 \mathrm{~mm}$ for: a) droplet and gas axial velocity, b) droplet mass flux rate. 92 Fig. 4.9 Same caption as Fig. 4.8, except for $x=36 \mathrm{~mm}$........................................... 94

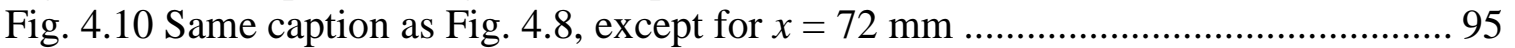

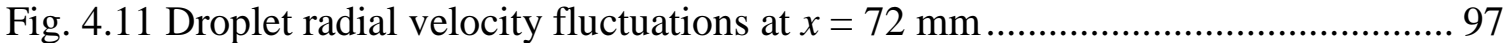
Fig. 5.1 Sketch of off-shore wind turbine system: a) conventional with electrical generator in nacelle on top, b) hydraulic-electric with electrical generator at base, and c) hydraulic-electric generator system with energy storage in tower ............................ 101 Fig. 5.2 Tower shapes and dimensions: height $H$, diameter $d$, and wall thickness $t \ldots . .104$ Fig. 5.3 The impact of tower mass savings (by reducing tower thickness and/or diameter) on tower frequency for three different head weights: i) baseline head weight described by thick solid lines, ii) head weight with $33 \%$ reduction described by thin solid lines and iii) head weight with $50 \%$ reduction described by dashed lines. The solid and hollow symbols indicate the tower mass savings when maintaining the baseline tower natural frequency coupled with a 1.4 safety factor $\left(1.4 f_{1 \mathrm{p}}\right)$ for respective head weight reductions of $33 \%$ and $50 \%$. Note that the rotor rated frequency $\left(f_{R}\right)$ is given by black solid line. 109 Fig. 5.4 Tower safety requirement if compressed air is stored inside a $5 \mathrm{MW}$ system .. 112 Fig. 5.5 Storage time for different rating power 113

Fig. 6.1 Sketch of a) axisymmetric simulation domain, and b) grid resolution and

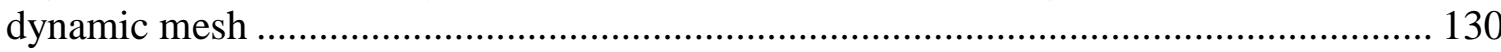

Fig. 6.2 Droplet position and size ................................................................... 133

Fig. 6.3 Contours of gas velocity magnitude with streamlines $[\mathrm{m} / \mathrm{s}]$ for equal total injected mass but different initial gas pressure 134

Fig. 6.4 Contours of droplet transient mass loading..... 135 Fig. 6.5 Transient mass loading normalized by total injected mass loading as a function of the piston travel (normalized $\%$ by chamber length) during compression (where $0 \%$

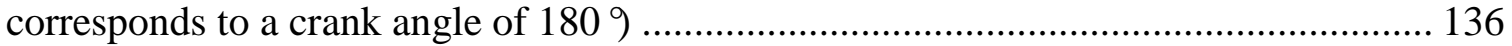
Fig. 6.6 Mean droplet size in the compression process ............................................ 138

Fig. 6.7 Total droplet surface area aloft at a given time ............................................. 140

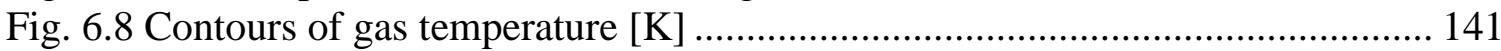

Fig. 6.9 Air temperature increase caused by compression.......................................... 142

Fig. 6.10 Pressure ratio as a function of piston volume during compression ................ 143

Fig. 6.11 Polytrophic index of compression process .................................................. 144

Fig. 6.12 Compression efficiency for different injected mass loading ........................ 144 
Fig. 7.1 Sketch of pre-mixed injection............................................................. 148 Fig. 7.2 Axisymmetric computational domain shown by red dashed box with inflow, wall and outflow boundary conditions for both validation case (based on Santolaya et al. geometry) and feeder tube cases .................................................................... 152 Fig. 7.3 Mean radial velocity comparison between CFD and experiment: a) at $x=18 \mathrm{~mm}$,

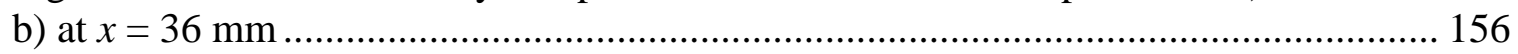

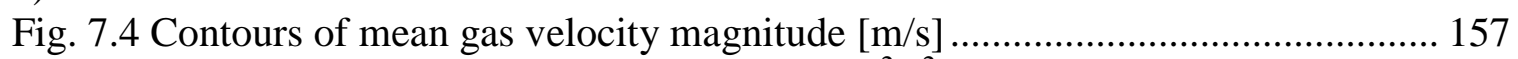

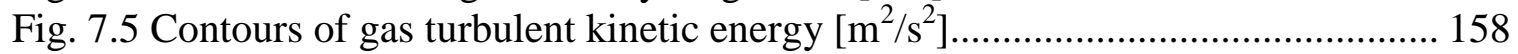

Fig. 7.6 Particle positions colored by velocity magnitude $[\mathrm{m} / \mathrm{s}]$ : a) $5-\mathrm{cm} \mathrm{b)} 8-\mathrm{cm}$ diameter

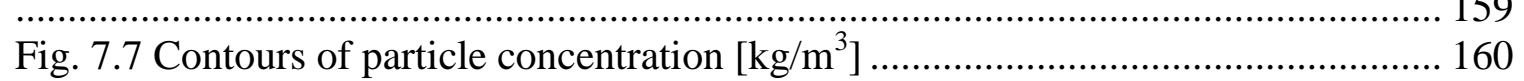

Fig. 7.8 Normalized droplet flow rate as a function of feeder tube axial position ......... 161 


\section{List of Tables}

Tab. 3.1 Characteristics of three-stage compression arrangement for a $5 \mathrm{MW}$ offshore wind energy storage system (independent of the degree of spray heat transfer) 54

Tab. 3.2 Performance characteristics of the proposed spray heat transfer three-stage compression design as described by Fig. 3.6 and Tab. 3.1....

Tab. 3.3 Comparison of traditional $5 \mathrm{MW}$ offshore wind turbine to a system that adds spray-based compressed air energy storage.....

Tab. 5.1 Characteristics and properties of the NREL 5 MW off-shore wind turbine..... 106

Tab. 5.2 Primary mode tower frequencies (in $\mathrm{Hz}$ ) as computed by computational methods and by theoretical method for $1^{\text {st }}$ mode $\left(f_{1}\right)$ and $2^{\text {nd }}$ mode $\left(f_{2}\right)$ 107 Tab. 5.3 Head weight comparison between conventional (electrical generator) and hybrid (hydraulic-electrical generators) systems 108 


\section{Chapter 1 Introduction}

Wind turbines and other unsteady power producing systems can benefit substantially by integrating inexpensive and easily sited Compressed Air Energy Storage (CAES) to provide a more continuous energy supply. However, traditionally CAES has not been combined with off-shore wind turbines. In addition, typical CAES systems are inefficient since the compression work also generates thermal energy, which is generally lost to the ambient when pressurized air is stored over a long period of time. To enable off-shore wind energy storage, a droplet spray heat transfer concept is investigated to establish a near-isothermal high-efficiency compression process. In particular, the use of small water droplets and high mass loading can allow for a large interfacial surface area for heat transfer. Issues associated with this liquid introduction can be mitigated with a liquid piston, which also allows variable piston cross-sections to further improve efficiency. To investigate the droplet spray heat transfer concept, a detailed multiphase thermodynamic model was developed and validated with experimental data. Based on this approach, one-dimensional simulations were performed using a sinusoidally driven piston in a 5kW first-stage cylinder with various compression ratios, as well as both premixed and direct injection scenarios. The results show that the total surface of aloft droplets is critical to achieve high performance in a liquid piston. This is best achieved with small droplets and high mass loadings combined with direct injection. For example, the compression efficiency (defined as a ratio of isothermal stored energy to real compression work) increased from $71 \%$ for adiabatic compression to as much as $98 \%$ with spray injection, for a ten-fold pressure. However, the effects of droplet collision (with other droplets and the chamber walls), three-dimensionality, injector dynamics, and 
the wall heat transfer should next be considered to help improve design and understanding of such systems.

Wind turbine output energy varies over time with local wind speed and is typically inconsistent with grid power demand. Without energy storage, the resulting difference between rated (peak) power and average power output leads to over-sizing of electrical generator and transmission lines. This conventional arrangement can be avoided if wind turbines can be coupled with energy storage to eliminate the output variations and instead produce their average power on a continuous basis. This would allow a smaller, lower-cost, constant-speed generator and a reduced capacity transmission system sized only for average power output. To accomplish this goal, it discusses a concept in Chapter 3 for a storage system for a 5MW off-shore wind turbine which integrates a spray-based compressed air energy storage with a $35 \mathrm{MPa}$ accumulator. The compressor employs a liquid piston for air sealing and employs water spray to augment heat transfer for high-efficiency. The overall compression is proposed in three stages with pressure ratios of $10: 1,7: 1$, and $5: 1$, all operated at $1 \mathrm{~Hz}$ to maintain moderate liquid surface acceleration. Based on a simple and fundamental description of the system, compression efficiency was found to be strongly dependent on droplet surface area, which can be achieved through either high mass loading or small drop sizes. The simulations also show that direct injection spray can increase overall three-stage compression efficiency to as high as $89 \%$, substantially better than the $27 \%$ associated with a conventional adiabatic compression at the same pressure ratio. In addition, this study introduces a key performance parameter, termed the Levelization Factor, which can be used to quantify the impact of storage on wind energy systems. However, experiments 
and simulations based on 3-D geometries with design details are needed to determine the potential of this concept.

Pressure-swirl nozzles are widely used in both industrial and experimental processes. However, the spray flow fields are difficult to predict due to the complexities of the dense break-up region. In Chapter 4, an inflow boundary condition approach is employed whereby droplets are computationally injected at $9 \mathrm{~mm}$ downstream of the orifice based on experimentally derived descriptions of the mean droplet and gas velocities, combined with empirical relations for turbulent kinetic energy and dissipation. The gas flow is then predicted with a Reynolds-Averaged Navier-Stokes solution while the particle trajectories are computed with a discrete random walk model. This approach was found to reasonably describe downstream spatial distribution of gas and droplet velocity, with moderate mesh resolution and CPU time. In addition, the evolution of the droplet velocity fluctuations is found to be consistent with turbulent diffusion theory based on the local Stokes numbers and turbulent kinetic energy.

While the growing size of wind turbines has strong advantages of increased wind speeds at higher altitudes and the larger rotor sizes that can capture more energy, the capital costs of wind turbine manufacture and assembly are substantial and increase as rotor diameter increases. A particular issue is the cost and placement of the electric generator in the nacelle at these large scales, which can be particularly problematic for off-shore wind turbines where access for maintenance and repair becomes more complex. In addition, increased rotor size translates to reduced rotor $\mathrm{rpm}$ and thus more complicated gearbox requirements. These issues may be addressed by introducing a hydraulic motor in the nacelle to convert rotor shaft work into hydraulic power that can 
be transmitted to the electric generator at ground/sea level. This combination of hydraulic and electric power generation can greatly reduce the nacelle/drive components, help simplify or even eliminate the gearbox to reduce maintenance costs, and greatly reduce the head weight mass that the tower needs to support. In addition, this hybrid concept allows the use of energy storage in the tower itself which can reduce the size of the electric generator for a given average power generation. To quantify the benefits of a hybrid hydraulic-electric power generation and of possible energy storage, the National Renewable Energy Laboratory (NREL) off-shore 5-MW baseline wind turbine was employed as a baseline in terms of tower mass and design in Chapter 5. Tower flexural natural frequencies based on both theory and industrial software packages were found to be within $3 \%$ of NREL design values. Based on an estimated head weight reductions of $33 \%$ and $50 \%$ and maintaining the tower natural frequencies and diameter, the tower mass can be reduced by a similar amount through decreased tower thickness. Even higher tower mass savings can be realized for larger turbines based on theoretical scaling. In addition, the hydraulic-electric generator concept is compatible with compressed air/liquid energy storage in the tower. Analysis based on cross-over pressure for the design limit indicates that this energy storage concept provides only a small benefit for steel towers, but can store as more than 24 hours of energy if one considers S-glass towers of $8 \mathrm{MW}$ or more.

Integrating Compressed Air Energy Storage (CAES) to a power supply system with a variable and unsteady energy source can bring a number of advantages. For offshore wind turbines, a novel approach to compressed air energy storage was recently proposed that allows high storage efficiency. This concept involves a liquid piston 
utilized as a compressor and expander with water spray to promote heat transfer to allow near isothermal processes. This concept has been previously investigated with onedimensional simulations that indicated spray cooling with droplet heat transfer over a large total surface area allows high-efficiency compression. However, the actual application is more complicated, and therefore the present study examines this concept with detailed two-dimensional flow. In Chapter 6, multi-phase computational fluid dynamics is implemented in an axisymmetric domain to investigate compression in a cylinder for first-stage and second-stage compression, using a spray discharge within the cylinder at various mass loadings. The spray was generally achieved by operating a single pressure-swirl nozzle directed along the centerline and operating at the maximum liquid mass flux capable that still permitted a small average droplet diameter (of about 30 microns). The two-dimensional simulations captured novel flow characteristics such as vortex formation for the air flow near the cylinder head and strong spatial variations in droplet size and concentration. Despite these effects, the overall two-dimensional efficiency was similar to that of one-dimensional predictions. The results indicating that a single pressure-swirl nozzle injection resulted in an injected mass loading of 1.6 and yielded an efficiency as high as $93 \%$ for a first-stage compression cycle. However, this same nozzle only resulted in injected mass loading of only 0.185 for a second-stage compression cycle (with an intake pressure of 10 bar), which resulted in reduced overall work efficiency. To satisfy both high mass loading and uniform distribution for secondstage operation, a multi-nozzle configuration should be considered.

Studies have shown that spray cooling can enhance liquid piston compression performance based on a large number of small droplets so as to provide a large surface 
area for heat transfer. Chapter 6 focuses on direct injection approaches to inject droplets into the pressure chamber, but a pre-mixed approach may serve to increase the degree of mixing and the mass of drops that can be injected. To examine a pre-mixed approach quantitatively, it is important to consider the spray interaction in the pre-mixed feeder tube. In Chapter 7, multi-phase flow simulation is implemented in an axisymmetric domain to investigate spray injection in a feeder tube to examine effects of droplet concentration uniformity and wall loss rate as a function of feeder tube diameter and length. The present computational method includes a Reynolds-Averaged Navier-Stokes approach for the turbulent gas phase coupled with a random walk model approach for turbulent diffusion of the droplet phase. This computational method was first validated with a single pressure-swirl nozzle with a weak gas co-flow and the results demonstrated reasonable agreement. This approach was then applied to two different feeder tube geometries (based on two different diameters) for an injected mass loading of unity. It was found that a feeder tube with a diameter of $8 \mathrm{~cm}$ and length of $30 \mathrm{~cm}$ performs and provides a reasonably uniform droplet radial distribution with less than $5 \%$ droplet loss to the walls. 


\section{Chapter 2 Liquid Piston Compression with Droplet Heat Transfer}

\subsection{Introduction}

\subsubsection{Energy Storage}

Compressed Air Energy Storage (CAES) emerged during the 1970s for peak shaving usage ${ }^{[1]}$ and was proven as a successful technology at the Huntorf ${ }^{[2]}$ and McIntosh ${ }^{[3]}$ plants. When power generation and power demand have different temporal profiles, in order to maintain grid stability and to user's demand, a CAES system can help provide a flexible balancing power supply. Due to its large-scale, long-duration storage, and fast output response time ${ }^{[4]}$, CAES can be particularly helpful for sources with large variations in power generation, such as wind turbines ${ }^{[5]}$. In a typical hybrid CAES and wind energy system ${ }^{[6]}$, a traditional gas turbine cycle is altered and separated into compressor portion and expander/generator portion. In the compression portion, off-peak or excess electricity generated by wind turbines is used to drive air compressors to compress ambient air, which is then stored in a reservoir, such as an underground natural cavern. Subsequently, when electricity demands are high but the wind is not strong, the compressed air is released and heated through a recuperator. The stored energy is extracted by burning with combustible fuel and expanding in a turbine.

As shown in this study, CAES technology for wind turbines can also help eliminate over-capacity design that is required to accommodate fluctuations in the delivered power due changes in wind speed. With CAES, the transmission lines and equipment with energy storage can be sized for the average power, rather than the maximum power level (which is often three times greater for wind turbines). However, 
the above hybrid CAES is difficult for off-shore wind turbines because of two reasons: 1) there is typically not an underground cavern off-shore; 2) operation of an off-shore gas turbine is difficult and expensive. Thus, CAES design requires special adaption for offshore wind energy systems. The proposed design employs an open accumulator and a compressor/expansor to increase energy density. The use of a hydraulic motor at the hub combined with the storage system and a downstream electric generator can further reduce the overall cost of energy since steady power at fixed RPM can be delivered to the mechanical-electric generator. This allows the generator to operate at a fixed speed and torque that increases its efficiency while reducing its weight, complexity, and cost. Furthermore, shifting the generator from the turbine nacelle to the turbine base platform eliminate the need for a complex gear box or variable drive system ${ }^{[7,8]}$. However, a net cost-of-energy advantage is only realized if the energy storage and extraction have very high efficiency. This study focuses on a novel method to increase compressed gas energy storage in order to help realize all the above advantages.

\subsubsection{Enhanced Heat Transfer by Droplets}

Conventional pneumatic compression using large-scale piston components typically incurs a large increase in gas temperature. This is because the rapid compression, which typically is less than a second, yields a nearly adiabatic process due to minimal heat transfer. This resulting increase in thermal energy consumes more compression work than an isothermal process. This is a problem because the thermal energy will generally be lost through external heat transfer when the gas is stored in a high-pressure accumulator over long periods, e.g., several hours. As a result, the overall compression efficiency is reduced especially at high pressure ratios. For example, a 
compression ratio of 256 results in $500 \mathrm{MJ} / \mathrm{m}^{3}$ of thermal energy lost for an adiabatic process as compared to an isothermal process ${ }^{[9]}$. To circumvent the associated reduction in efficiency, one can instead employ a high enough heat transfer to achieve nearly isothermal compression. A droplet spray can be an effective solution since the large number of small size droplets can provide a high total surface area for heat transfer ${ }^{[10-12]}$. However, the presence of significant liquid volume in the piston chamber must also be accommodated. One solution is to use a liquid piston, which has other benefits as described in next section.

Compared to the traditional approaches of enhancing heat transfer during compression or expansion such as water jackets, droplet heat transfer can be applied in the interior of the gas chamber and hence can be much more direct and effective. Therefore, spray cooling may be an excellent option for CAES since conventional nearadiabatic compression can be inefficient. This is particularly true for off-shore windturbine concepts which have an abundant source of water (to be used for the spray) at ambient temperature and where on-site storage can be used to dampen and even eliminate wind power fluctuations can be reasonably integrated into the overall platform.

\subsubsection{Liquid Piston}

In Ref. 13, Van de Ven and Li proposed a liquid piston concept aimed to improve the behavior and increase the efficiency of a gas compression and expansion. This concept replaces the traditional solid piston with a liquid column resting over the piston surface to directly compress gas in a fixed-volume vertically-oriented compression chamber. This has considerable advantages. Firstly, as proposed herein, liquid pistons can readily accommodate water spray cooling during compression or expansion since the 
droplets can simply fall into the liquid interface. Secondly, a liquid piston can allow for a variable cross-sectional geometry at the liquid-gas interface while keeping a fixed crosssectional geometry along the solid piston stroke. This variability of geometry at the compression surface can be used to incorporate higher-efficiency non-sinusoidal volumetric changes while maintaining a simple sinusoidal stroke for the solid piston interface $^{[13]}$. Thirdly, it can also be used to increase surface area and thus heat transfer by introducing a matrix of longitudinal tubes (along the stroke direction) within the compression cylinder, but above the maximum height achieved by solid piston. In addition, Li et al. ${ }^{[14]}$ showed that such a liquid piston concept integrated into a compact fluid energy storage system can have significant advantages for offshore wind turbine systems by making use of the surrounding water as a heat sink. Fourthly, the liquid column can minimize air leakage.

\subsubsection{Objectives and Motivation}

Herein, one-dimensional simulations of liquid piston compression with droplet heat transfer are completed to determine the conditions required for high efficiency and the potential improvement in overall performance. If such storage can be done efficiently, this can help solve issues associated with temporal mismatch of wind turbine power generation (which typically peaks at night) and electrical energy demand (which typically peaks in the day time).

To understand the influence and importance of the spray cooling, effects of droplet size, total mass injected, and water spray injection method are considered in this study. The test conditions used for the compression cycle are based on the first-stage of an off-shore wind-turbine CAES concept. To the author's knowledge, this is the first 
study to investigate in detail the spray cooling concept (e.g., in terms of mean droplet size, mass loading, injection mechanism) for compressed air energy storage in terms of overall efficiency. It is also the first to consider spray cooling in the context of off-shore windenergy storage.

\subsection{Overall Concept and Spray Injection Options}

For internal combustion engines, the two primary methods for fuel spray are: premixed injection and direct injection. Figures 2.1 and 2.2 are schematics of these two injection methods as proposed herein for a spray-cooled liquid piston concept. These figures show distinct cycle segments based on the crank angle $(\theta)$. The first is an intake portion where the ambient pressure air $\left(p_{\mathrm{atm}}\right)$ is pulled in over as theta changes from $-\pi \leq$ $\theta \leq 0$. The second is a compression portion for $0 \leq \theta \leq \theta_{\mathrm{CR}}$, where $\theta_{\mathrm{CR}}$ occurs when the chamber pressure reaches the maximum desired pressure, $p_{\mathrm{CR}}$. If the pressure losses due to inflow and outflow are ignored, the maximum pressure is based on the compression ratio, $\mathrm{CR}=p_{\mathrm{CR}} / p_{\mathrm{atm}}$. The last portion of the cycle is the exhaust $\left(\theta_{\mathrm{CR}} \leq \theta \leq \pi\right)$ where the high pressure air is displaced into the accumulator tank for energy storage. Note that the liquid piston is assumed to be vertical with droplet injection from above so that the gravity keeps the liquid column approximately horizontal and also pulls the drops downward.

For direct injection (Fig. 2.1), the spray can be continuously discharged within the chamber for both the intake and compression portions $\left(\theta_{\mathrm{CR}} \leq \theta \leq \pi\right)$ to maximize the droplet surface area available for heat transfer. As ambient-temperature droplets continuously enter the chamber, any increase in gas temperature causes a relative temperature difference. With direct injection, this difference can be maintained 
throughout compression and new droplets can be added after the intake valve is closed. However, direct injection requires that the spray supply pressure exceed the maximum gas pressure $\left(p_{\mathrm{CR}}\right)$, and that the nozzles be incorporated within the chamber head.

For pre-mixed injection (Fig. 2.2), water spray can be mixed with inlet gas upstream of the cylinder during the intake portion. This allows a more modest spray supply pressure and helps fill the entire chamber with droplets at the start of the compression process. However, droplets can no longer be injected when the compression portion begins and thus the droplet surface area will continually decrease as the droplets fall into the liquid piston interface.

In both configurations, the total amount of water injected can be expressed in terms of the mass loading, defined as the ratio of water spray mass injected in a cycle $\left(m_{\text {spray }}\right)$ to the mass of dry air drawn into the piston chamber $\left(m_{a}\right)$, i.e.

$$
M L=\frac{m_{\text {spray }}}{m_{a}}
$$

The heat transfer will be proportional to the instantaneous mass of water available and its surface area, which is a function of the droplet diameter $(d)$. To understand how the droplet diameter and mass loading impact the efficiency of the concept, one must model the droplet heat transfer, which is a function of the particle relative velocity and its mass transfer rate. 

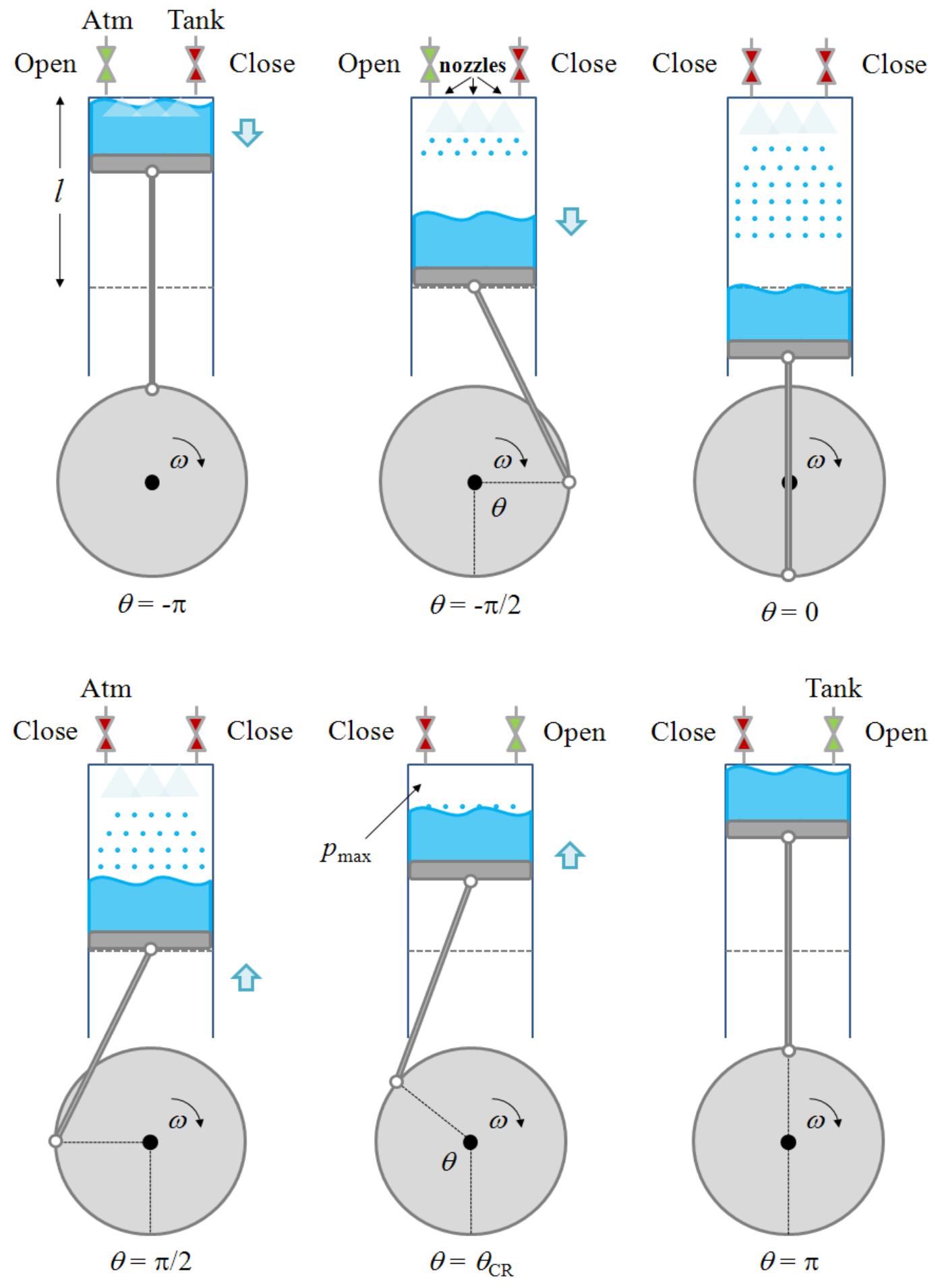

Fig. 2.1 Concept for direct injection of water droplets with a liquid piston 

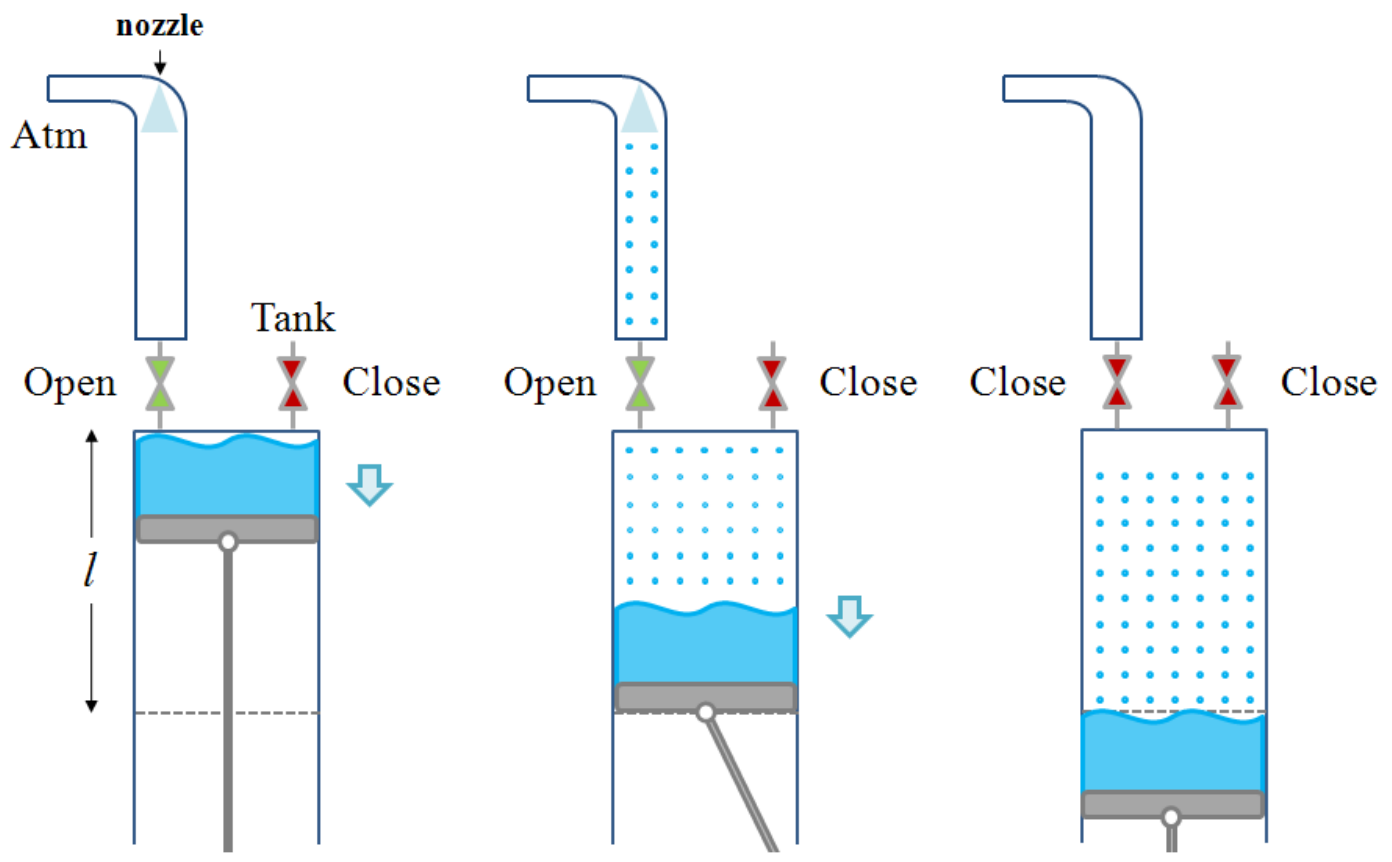

$$
\theta=-\pi
$$

$\theta=-\pi / 2$
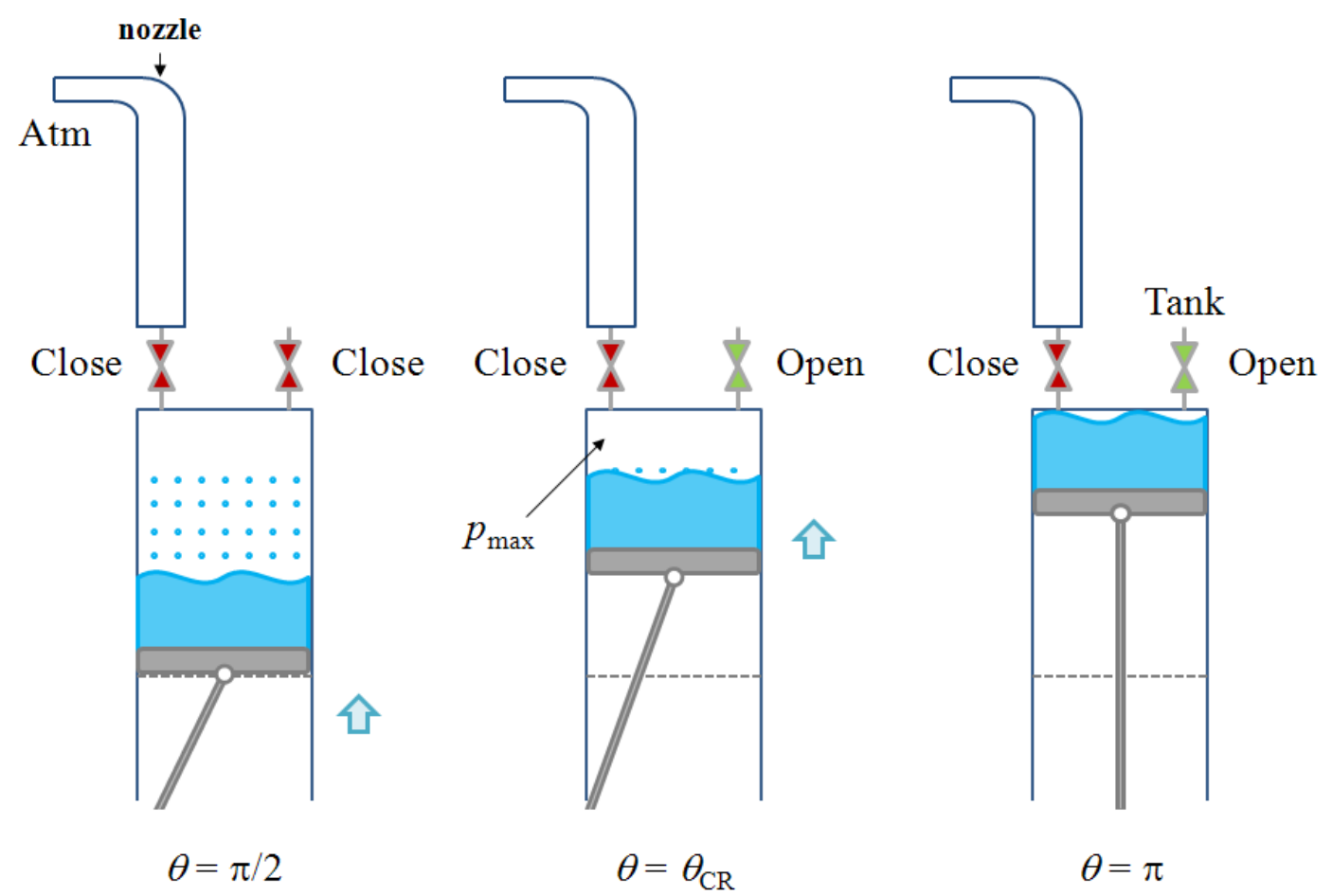

Fig. 2.2 Concept for pre-mixed injection of water droplets in an upstream passage 


\subsection{Flow Physics}

\subsubsection{Piston and Gas Velocity Field}

In the following, a one-dimensional analysis is employed to assess the basic concepts of the spray-cooled liquid piston system. The movement of the liquid piston interface is determined by assuming that the solid piston below is driven by a simple rocker which creates a sinusoidal displacement (as shown in Figs. 2.1 and 2.2). The onedimensional position and velocity of the liquid piston interface and the droplet trajectories can be shown in Fig. 2.3. The coordinate system in this figure defines positive $\mathrm{z}$ as downwards where $\mathrm{z}=0$ corresponds to the top of the compression chamber, and $z_{P}$ corresponds to the piston interface. For a given piston of the stroke length $(l)$ and the cycle frequency $(f)$, the position $\left(z_{P}\right)$, velocity $\left(u_{P}\right)$, and acceleration $\left(a_{P}\right)$ of the liquid piston interface are given as a function of time $(t)$ as

$$
\begin{aligned}
& z_{P}=\frac{l}{2}[1+\cos (2 \pi f t)] \\
& u_{P}=-l \pi f \sin (2 \pi f t) \\
& a_{P}=-2 l \pi^{2} f^{2} \cos (2 \pi f t)
\end{aligned}
$$

Higher piston speeds and accelerations allow a more compact system for a given power level. However, for a vertical liquid piston, the acceleration should be limited relative to gravitational acceleration $\left(g=9.8 \mathrm{~m} / \mathrm{s}^{2}\right)$ to avoid the liquid interface

instabilities ${ }^{[13,14]}$. As such, an approximate limit is that the maximum value of $a_{P}$ is bounded by $1 / 2 \mathrm{~g}$. This translates to a maximum piston frequency of 


$$
f_{\max } \sim \frac{1}{2 \pi} \sqrt{\frac{g}{l}}
$$

For this condition, the cycle frequency is inversely limited by the stroke length. Assuming a constant ratio of stroke length to piston diameter and a fixed compression ratio, this limit therefore also specifies the energy storage for a given piston size.

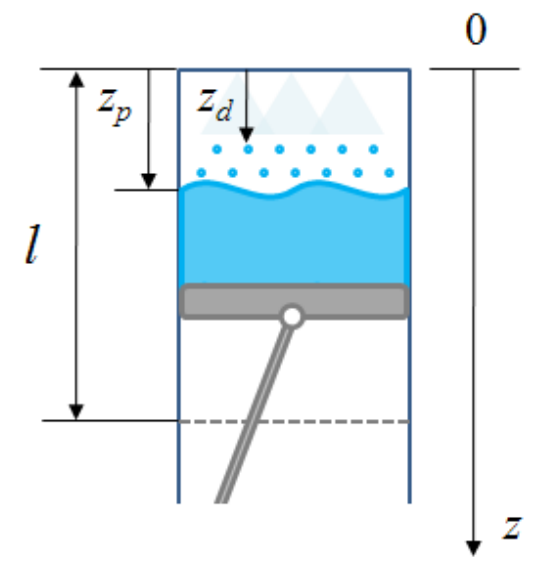

Fig. 2.3 Vertical coordinate system for positions of droplets and liquid piston interface

To determine the one-dimensional gas velocity as a function of time, one may reasonably assume a linear distribution from the top of chamber $(\mathrm{z}=0)$ where the gas velocity is zero, to the liquid interface $\left(z_{P}\right)$ where the gas velocity is as same as the piston velocity $u_{P}$, i.e.

$$
u=\frac{z}{z_{p}} u_{p}
$$

Aside from asymmetries due to boundary layer development and intake geometry, Eqs. 2-3 and 2-4 were found to be reasonable for cylindrical pistons using detailed computational fluid dynamic studies. 


\subsubsection{Droplet Velocity Field}

Spray cooling is a two-phase phenomenon complicated by mass, momentum and thermal dynamics of both the drop and the gas (including its level of humidity), which typically require empirical analyses ${ }^{[15,16,17]}$.

For the droplet momentum equations, the drag and gravity forces are expected to dominate given the high density ratio between the liquid and the gas ${ }^{[15]}$. Thus, the equation of motion for the droplet with centroid velocity $(v)$ and mass $\left(m_{d}\right)$ can be expressed in terms of the drop position $\left(z_{d}\right.$, based on Fig. 2.3), drop relative velocity $(\boldsymbol{w})$ and drop diameter $(d)$ as

$$
\begin{aligned}
& \frac{d\left(m_{d} \boldsymbol{v}\right)}{d t}=-3 \pi \mu_{g} F(\boldsymbol{v}-\boldsymbol{u}) d+m_{d} \boldsymbol{g} \\
& m_{d}=\frac{\pi}{6} \rho_{d} d^{3} \\
& v \equiv \frac{d z_{d}}{d t} \\
& \boldsymbol{w} \equiv \boldsymbol{v}-\boldsymbol{u}
\end{aligned}
$$

In the first expression, $F$ is the Stokes correction factor, $\mu \mathrm{g}$ is the gas dynamic viscosity, $\rho$ is the air density, and the subscripts $g$ and $d$ represent gas and droplet respectively.

For a one-dimensional motion, the droplet acceleration becomes

$$
\frac{d v}{d t}=-\frac{18 \mu_{g} F}{\rho_{d} d^{2}} w+g
$$


Generally, $F$ is a function of droplet shape and Reynolds number $\left(\operatorname{Re}_{\mathrm{d}}\right)$, defined as

$$
\operatorname{Re}_{d}=\frac{\rho_{g}|w| d}{\mu_{g}}
$$

The shape can be assumed to be approximately spherical for diameters of 400 microns or less ${ }^{[15]}$ as is considered herein, so the shape effects can be reasonably ignored. However, Reynolds number effects can be significant. A common empirical description of this effect for a fixed-diameter sphere is given by ${ }^{[18]}$ as

$$
F_{0}=1+\frac{\operatorname{Re}_{d}}{4\left(1+\sqrt{\operatorname{Re}_{d}}\right)}+\frac{\operatorname{Re}_{d}}{60} \text { for } \operatorname{Re}_{d} \leq 2 \times 10^{5}
$$

If the gas is not saturated, then evaporation effects can reduce drag force due to the presence of is a vapor mass flux from the droplet surface and the boundary layer near the droplet surface has been changed by this effect, a semi-empirical correlation ${ }^{[12,19]}$

$$
F=\frac{F_{0}}{1+B}
$$

where $B$ is called the Spalding mass number,

$$
B=\frac{\omega_{v, \text { surf }}-\omega_{v}}{1-\omega_{v}}
$$

where $\omega_{v}$ is the vapor mass fraction in the gas, and $\omega_{v, \text { surf }}$ is the vapor mass fraction near the droplet surface based on the droplet surface temperature and assuming saturated conditions. The correction associated with Eq. 2-9 in the present conditions is generally limited to $5 \%$ or less. 
The above drag corrections and momentum equation can be employed to describe the droplet motion for a given set of initial conditions. However, drops with diameters of 50 microns or less will have a velocity response time of 0.03 seconds or less which is much smaller than the cycle period of about 1 second (for intake and compression strokes). As such, it is reasonable to assume that such drops with low injection velocities (as in the direct injection case) will come to relative velocity equilibrium relative to the gas $^{[18]}$. This difference will be equal to the terminal velocity which can be obtained from Eq. 2-6 as

$$
\begin{aligned}
& w_{\text {term }}=\frac{\left(\rho_{d}-\rho_{g}\right) g d^{2}}{18 \mu_{g} F_{\text {term }}} \\
& F_{\text {term }}=\left[1+0.76 \operatorname{Re}_{d}\left(1+0.025 \operatorname{Re}_{d}\right)\right]^{0.18} /(1+B)
\end{aligned}
$$

The terminal Stokes correction factor $\left(F_{\text {term }}\right)$ is based Ferry and Balachandar ${ }^{[20]}$, modified herein to include the mass transfer effect.

\subsubsection{Interphase Mass Transfer}

The droplet mass transfer rate (defined as positive from the gas to the drop) can be expressed as a function of Spalding mass number

$$
\frac{d m_{d}}{d t}=-\operatorname{Sh} \rho_{g} \mathcal{D} \pi d \ln (1+B)
$$

where Sh is Sherwood number and $D$ is diffusion coefficient. Referring the RanzMarshall equation, the Sherwood number for a sphere is given by

$$
\mathrm{Sh}=2+0.6 \mathrm{Re}^{1 / 2} \mathrm{Sc}^{1 / 3}
$$


with Schmidt number $(\mathrm{Sc})$ defined as

$$
\mathrm{Sc}=\frac{\mu_{g}}{\rho_{g} \mathcal{D}}
$$

Combining the above equations (2-12 to 2-14) with Eq. 2-5b, the diameter variation in time can be expressed in the form of an Ordinary Differential Equation (ODE) as

$$
\frac{d(d)}{d t}=-\frac{2 \operatorname{Sh} \rho_{g} \mathcal{D}}{\rho_{d} d} \ln (1+B)
$$

If the gas humidity is high, the vapor mass fraction difference between the neardrop-surface and the surrounding is small (i.e. $B \approx 0$ ), which indicates that the rate of mass transfer (and of diameter change) will also tend to zero.

\subsubsection{Interphase Heat Transfer}

The rate of heat transfer (defined as positive from the gas to the droplet) for a single drop is

$$
\dot{q}=\mathrm{Nu} k_{g} \pi d\left(T_{g}-T_{d}\right)
$$

In this expression, $k_{g}$ is the thermal conductivity of gas, $T_{g}$ is the uniform gas temperature, and $\mathrm{Nu}$ is the Nusselt number. Based on the assumption that the surface temperature of moving droplets is fixed, the Nusselt number can be obtained from the well validated Ranz-Marshall relationship

$$
\mathrm{Nu}=2+0.6 \operatorname{Re}^{1 / 2} \operatorname{Pr}^{1 / 3} \text { for } \operatorname{Re} \leq 200, \operatorname{Pr} \leq 250
$$


where the Prandtl number (Pr) is a function of the specific heat capacity of the gas at constant pressure $\left(c_{p}\right)$ as

$$
\operatorname{Pr}=\frac{c_{p} \mu_{g}}{k_{g}}
$$

The heat balance of the droplet can then be expressed as,

$$
m_{d} c_{d} \frac{d\left(T_{d}\right)}{d t}=\dot{q}+L \frac{d m_{d}}{d t}
$$

where $\mathrm{c}_{\mathrm{d}}$ is the specific heat capacity of droplet and $L$ is the latent heat of vaporization. At the Reynolds numbers associated with the present application, this droplet-surface-temperature-fixed model is quite accurate ${ }^{[21]}$. In Appendix A, a validation is given which shows the deviation between this model and the experiment is small.

Based on Eq. 2-19, we can define droplet thermal response time $\left(\tau_{\mathrm{q}}\right)$ and reference temperature $\left(\mathrm{T}_{\mathrm{q}}\right)$ as

$$
\begin{aligned}
\tau_{q} & =\frac{\rho_{d} d^{2} c_{d}}{6 \mathrm{Nu} k_{g}} \\
T_{q} & =\frac{L \operatorname{Sh} \rho_{g} \mathcal{D} \ln (1+B)}{\operatorname{Nu} k_{g}}
\end{aligned}
$$

The above equations $(2-19,2-20)$ can be combined with Eq. 2-5b to give an ODE for the droplet temperature as 


$$
\frac{d T_{d}}{d t}=\frac{T_{g}-T_{d}}{\tau_{q}}-\frac{T_{q}}{\tau_{q}}
$$

The corresponding heat balance for the gas phase can then be expressed as

$$
m_{g} c_{p} \frac{d T_{g}}{d t}=\sum_{\mathrm{j}}^{N_{d}}\left[-\dot{q}+h_{d}\left(1-\omega_{v}\right) \frac{d m_{d}}{d t}\right]_{\mathrm{j}} \equiv-\dot{Q}
$$

where $N_{d}$ is the instantaneous number of droplets aloft in the gas and $h_{d}$ is droplet enthalpy. Thus, the gas temperature change can be written as

$$
\frac{d T_{g}}{d t}=-\frac{\dot{Q}}{m_{g} c_{p}}
$$

Assuming that the cylinder walls are adiabatic and the drops are distributed uniformly, the gas temperature can be assumed to be only a function of time (and not space).

\subsection{Numerical Treatment}

For steady case, gas and liquid phase ordinary differential equations are integrated in time with a finite difference approach, similar to the approach used by Hiestand et al. ${ }^{[22]}$ for the exhaust flue gas and water droplets in a humidification process and based on the particle-source-in-cell model of Crowe et al. ${ }^{[23]}$. While there are limited studies related to that droplet heat transfer in a closed chamber during compression, the study by Sazhin ${ }^{[24]}$ and Prommersberger ${ }^{[25]}$ includes Lagrangian ODE integrations for fuel droplet evaporation in a constant pressure combustion application. These previous studies were used to consider the droplets in an environment with variable humidity, pressure and temperature. 
For pre-mixed injection, the droplets can be reasonably assumed to fall at the terminal velocity relative to the local gas velocity. For the higher droplet speeds associated with direct injection, the droplets are injected from the top of the chamber with a velocity corresponding to the mass loading and flow rate and then evolve based on the equation of motion. This equation can be expressed in terms of the particle velocity response time, which can be obtained from Eq. 2-6 as

$$
\tau_{D}=\frac{\rho_{d} d^{2}}{18 \mu_{g} F}
$$

If one assumes that the particle response time $\left(\tau_{D}\right)$ is constant over a timestep $(\Delta t)$ one may integrate Eq. 2-6 to give a discrete change in relative velocity as

$$
w_{\mathrm{i}+1}=w_{\mathrm{i}} \exp \left(-\frac{\Delta t}{\tau_{D}}\right)+g \tau_{D}\left[1-\exp \left(-\frac{\Delta t}{\tau_{D}}\right)\right]
$$

Given this relative velocity, and the sinusoidal gas velocity from Eq. 2-4, the droplet position can be obtained using a second-order central-difference scheme as

$$
z_{d, \mathrm{i}+1}=z_{d, \mathrm{i}}+\left(v_{\mathrm{i}+1}+v_{\mathrm{i}}\right) \frac{\Delta t}{2}
$$

A similar discrete form of the drop size change due to mass transfer can be expressed as

$$
d_{\mathrm{i}+1}=d_{\mathrm{i}}-\frac{2 \mathrm{Sh}_{\mathrm{i}} \rho_{g, \mathrm{i}} \mathcal{D}_{\mathrm{i}}}{\rho_{d} d_{\mathrm{i}}} \ln \left(1+B_{\mathrm{i}}\right) \Delta t
$$


Assuming constant $\tau_{\mathrm{q}}$ over the timestep (again reasonable for modest $\operatorname{Re}_{\mathrm{p}}$ values), the discrete analytical expression for the

$$
T_{d, \mathrm{i}+1}=T_{g, \mathrm{i}}-\left(T_{g, \mathrm{i}}-T_{d, \mathrm{i}}\right) \exp \left(\frac{-\Delta t}{\tau_{q, \mathrm{i}}}\right)-T_{r, \mathrm{i}}\left[1-\exp \left(\frac{-\Delta t}{\tau_{q, \mathrm{i}}}\right)\right]
$$

For an accurate time integration of these equations for the drop properties, the timestep should be small compared to the time-scale for momentum and thermal changes, i.e. $\Delta \mathrm{t}<<\tau_{\mathrm{D}}$ and $\Delta \mathrm{t} \ll \tau_{q}$. Since $\tau_{\mathrm{D}}<\tau_{q}$, the former criterion is most important. Herein, it was found that a $\Delta \mathrm{t}=0.02 \tau_{\mathrm{D}}$ was sufficient to yield drop positions and velocities converged to within $99 \%$ accuracy.

For the gas in the chamber, a two-step approach is used to update the temperature and pressure for each timestep. The gas is first assumed to be compressed adiabatically due to the piston movement; then the heat and mass transfer are imparted between droplets and the gas, to determine the temperature at the next timestep. For the first step, adiabatic compression with the ideal gas law yields the change in temperature due to the change in piston volume $(V)$

$$
T_{g, \mathrm{i}+1}^{\prime}=\left(\frac{V_{\mathrm{i}}}{V_{\mathrm{i}+1}}\right)^{\gamma-1} T_{g, \mathrm{i}}
$$

where $\gamma$ is the ratio of specific heats. The prime superscript indicates that it is an intermediate temperature in the computational scheme. Next, the heat transfer between the gas and the droplet is incorporated based on Eq. 2-23 as

$$
T_{g, \mathrm{i}+1}=T_{g, \mathrm{i}+1}^{\prime}-\frac{\dot{Q} \Delta t}{m_{g} c_{p}}
$$


Employing the ideal gas law, the new gas pressure in the chamber can be obtained. The timestep integration is repeated until the pressure reaches the desired maximum value, after which the exhaust valve is opened and the pressure is assumed constant while the piston pushes the compressed gas into the accumulator. The methodology described above was validated with experimental data for spray-cooling in a channel flow as described in the Appendices.

\subsection{Test Conditions for Off-Shore Wind Turbines}

The test conditions investigated are consistent with one chamber of the first stage in a matrix of liquid pistons. In particular, a cylinder compression chamber with an inner diameter of $0.3 \mathrm{~m}$, a length of $0.3 \mathrm{~m}$, and a compression ratio is 10 are employed. Based on Eq. 2-3, this yields a maximum cycle frequency of $1 \mathrm{~Hz}$ corresponding to a storage power of $5 \mathrm{~kW}$. A $5 \mathrm{MW}$ turbine would employ hundreds of these chambers for a firststage compression, and similar chambers for second- and third-stage compression ${ }^{[13,14]}$. A range of compression ratios (from 6 to 12) is also considered, which are reasonable for the first stage. The intake air was assumed to have an air temperature of $20^{\circ} \mathrm{C}(293.15 \mathrm{~K})$ a pressure of $101 \mathrm{kPa}$ and a water vapor volume fraction of $1.3 \%$, which represents a relative humidity of $8.9 \%$.

For the spray, mean droplet diameters of 10, 20, 50, and 100 microns were considered since these are reasonably achievable by most water spray nozzles. Mass loadings considered include $0.5,1,2$, and 5 , though it is noted that mass loadings greater than one would generally require multiple injectors. The initial water temperature is the same as that of the intake air, i.e. $20^{\circ} \mathrm{C}$ or $293.15 \mathrm{~K}$. 


\subsection{Results and Discussions}

\subsubsection{Heat Transfer Characteristics}

The evolution of the gas temperature, pressure, and time-integrated work during the compression process reflects the droplet effects on enhancing internal heat transfer. Figures 2.4 and 2.5 show the impact of the total water spray discharged into the chamber on the evolution of these three compression characteristics for various mass loading with a droplet diameter of 20 microns. Temperature and pressure increase as the liquid piston travels towards the desired maximum pressure value. Once this pressure and crank angle is reached (at $p=p_{C R}$ and $\theta=\theta_{C R}$ ), the pressure thereafter stays constant and further piston work is used to push the compressed air out of the chamber. For each case, the evolution of the characteristics with a spray is compared to two limit boundaries: adiabatic conditions (where no heat transfer occurs) and isothermal conditions (the ideal limit of maximum heat transfer).

As can also be observed from the line " $\theta=\theta_{\mathrm{CR}}$ " in Figs. 2.4a and 2.5a, it takes longer to reach the desired pressure as the flow tend to the isothermal condition. This is because for the ideal gases at the same pressure, the lower temperature gas has smaller volume. The change in temperature between isothermal and adiabatic conditions for the compression ratio of ten is quite substantial (nearly $300 \mathrm{~K}$ ), indicating that heat transfer effects can play a major role in this system. In general, the results tend towards the isothermal condition as the mass loading (net mass of drops) increases indicating improved overall performance. This is especially true for the direct injection case (Fig. 2.5) with a mass loading of 5, where the characteristics curves closely approach the 
isothermal case. However, there is still substantial heat transfer even when for a ML=0.5 with pre-mixed injection, i.e. the final exhaust temperature is reduced by more than $100 \mathrm{~K}$.

To investigate the effects of droplet size, Fig. 2.6 shows the results for a fixed and intermediate mass loading $(\mathrm{ML}=1)$ for varying droplet diameters from 10 to 100 microns. Because of the fixed mass loading, the total mass of water droplet is constant, so the total number of injected droplets must increase as droplet diameter decreases. The result is a higher injected surface area as diameter decreases which is consistent with an increased tendency to the ideal isothermal condition. Conversely, the performance of large droplets, such as 100 microns, is not encouraging at this mass loading. However, the differences between 10 and 20-micron droplet cases are quite small compared to the differences between 20 and 50-micron in both injection methods. This is because the droplets are nearly in thermal equilibrium with the gas for drop diameters of 20 microns or less (since $\tau_{q}$ of Eq. 2-20a becomes negligibly small compare to the compression time-scale), so that additional surface area does not improve performance. Thus, the complexities and additional energy required to create the smaller 10 micron droplets will generally not be compensated by a performance increase. Thus, a 20 micron droplet is as a reasonable choice for the current application (and thus was used for Figs. 2.4 and 2.5).

Figure 2.7 shows that effective surface area of droplets aloft at any given time in the cylinder $(A)$ normalized by the total surface area injected $\left(A_{\text {inj }}\right)$. This ratio would reach $100 \%$ by the end of the injection if no droplets were lost. However, gravitational forces will induce a downward vertical velocity which will cause the droplets to fall into the liquid piston surface, after which their heat transfer influence will be negligible. For pre-mixed injection, all the droplets are injected at the beginning of the compression $(\theta=$ 
0) and the total droplet surface area decreases almost linearly as the terminal velocity causes them to continually fall into the liquid piston surface. For direct injection, the heat transfer area can be controlled throughout the compression since droplets can be continuously injected into the chamber. As shown in Fig. 2.7, direct injection with a uniform flux rate results in a net increase over time of the surface area, but it never reaches $100 \%$ because some droplets are still lost. However, it can be seen that direct injection allows a higher fraction of the injected droplets to be aloft during the critical portion of the compression cycle. For example, Fig. 2.6 shows that most of the temperature increase occurs for piston travel of $60 \%$ or more, and Fig. 2.7 indicates that direct injection allows more than twice the surface area (and three-fold the droplet mass) as compared to the pre-mixed condition. This increase in surface area and droplet mass at such times explains the increased tendency towards the isothermal conditions shown in Figs. 2.4-2.6 for the direct injection case. The next section quantifies this effect in terms of overall cycle efficiency.

\subsubsection{Efficiency Characteristics}

To quantify the performance of a spray-cooled liquid piston compression, the definition of stored energy, available work, and compression efficiency are introduced. In liquid piston compression, the air is compressed to a desired pressure and stored in an accumulator. While there is some viscous loss during the compression, the current conditions lead to laminar boundary layers such that the viscous work is less than $2 \%$ of the inviscid compression work, and can be reasonably neglected. The inviscid compression is based only on pressure work and can be written in integral form or approximately in discrete summation form as 


$$
W=\int\left(p-p_{a t m}\right) d V \approx \sum\left(p-p_{a t m}\right) \Delta V
$$

The right-hand-side expression assuming a constant gas pressure between each interval (taken here as the average) with $\Delta \mathrm{V}$ is the volume change in a time interval. If the time-step is sufficiently small, this form will converge to the integral definition.

To determine the energy stored by this process, one may assume that the compressed air will tend to the ambient temperature while in storage and then later will be expanded to atmospheric pressure isothermally. The maximum regenerated energy can thus be expressed as

$$
\mathcal{E}=\int\left(p_{C R}-p_{a t m}\right) d V=\rho_{a t m} V_{g 0} T_{a t m} \ln C R
$$

where $V_{g 0}$ is the gas volume after expansion, which is also equal to the gas chamber volume at $\theta=0$ by the ideal gas law (since mass is conserved).

The ratio of the ideal energy available to the compression work can then be defined as the compression efficiency

$$
\eta=\frac{E}{W} \times 100 \%
$$

For a viable CAES system with a fixed energy output, it is important that this efficiency is close to $100 \%$ since the compression chambers and system cost will scale with the total work input. Therefore, the compression efficiency is a primary performance parameter with which spray properties can be considered. 

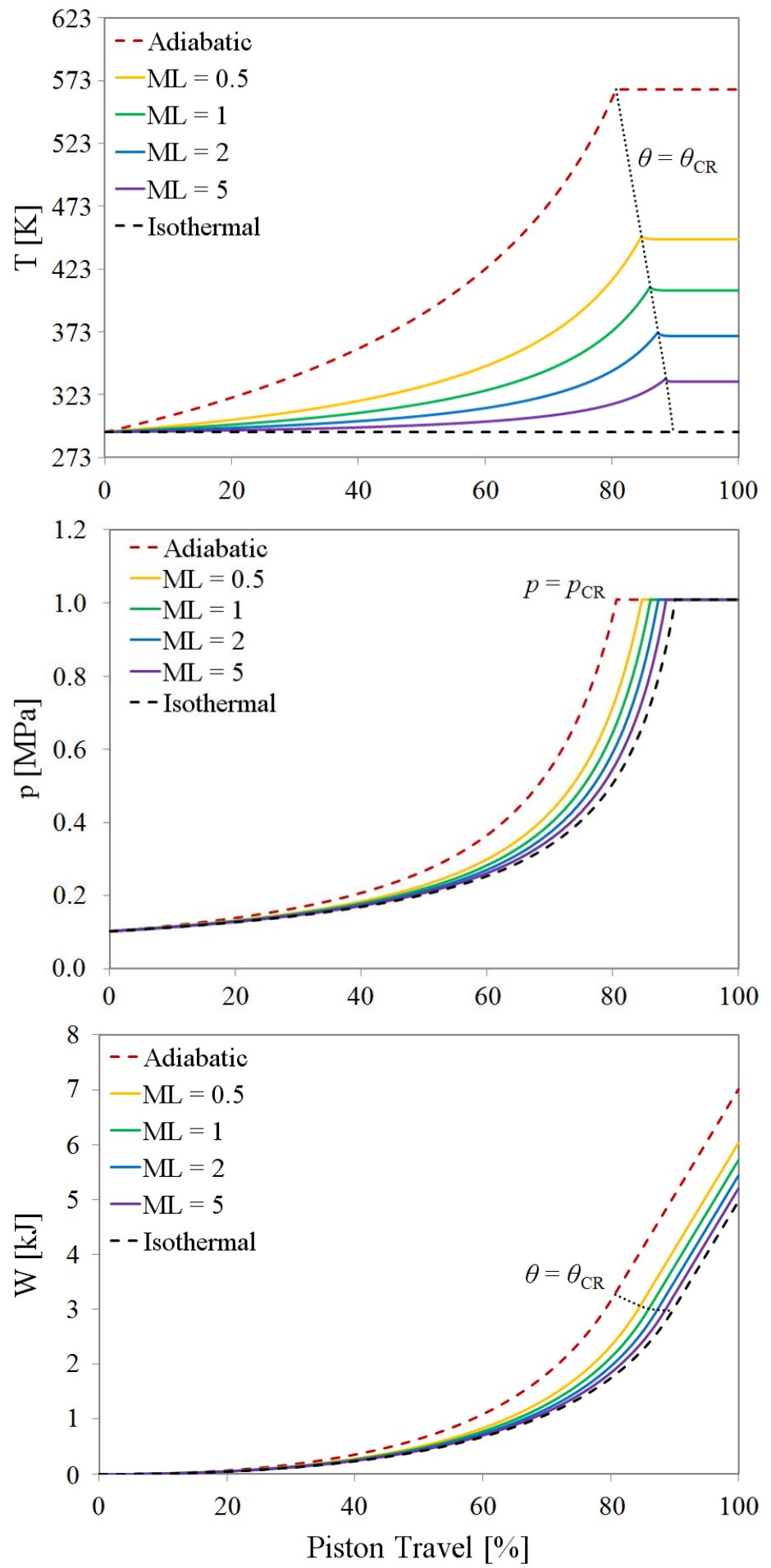

Fig. 2.4 Temperature, Pressure, and Compression Work vs. Piston Travel for Pre-mixed Injection $(\mathrm{d}=20 \mu \mathrm{m}, \mathrm{CR}=10)$ 

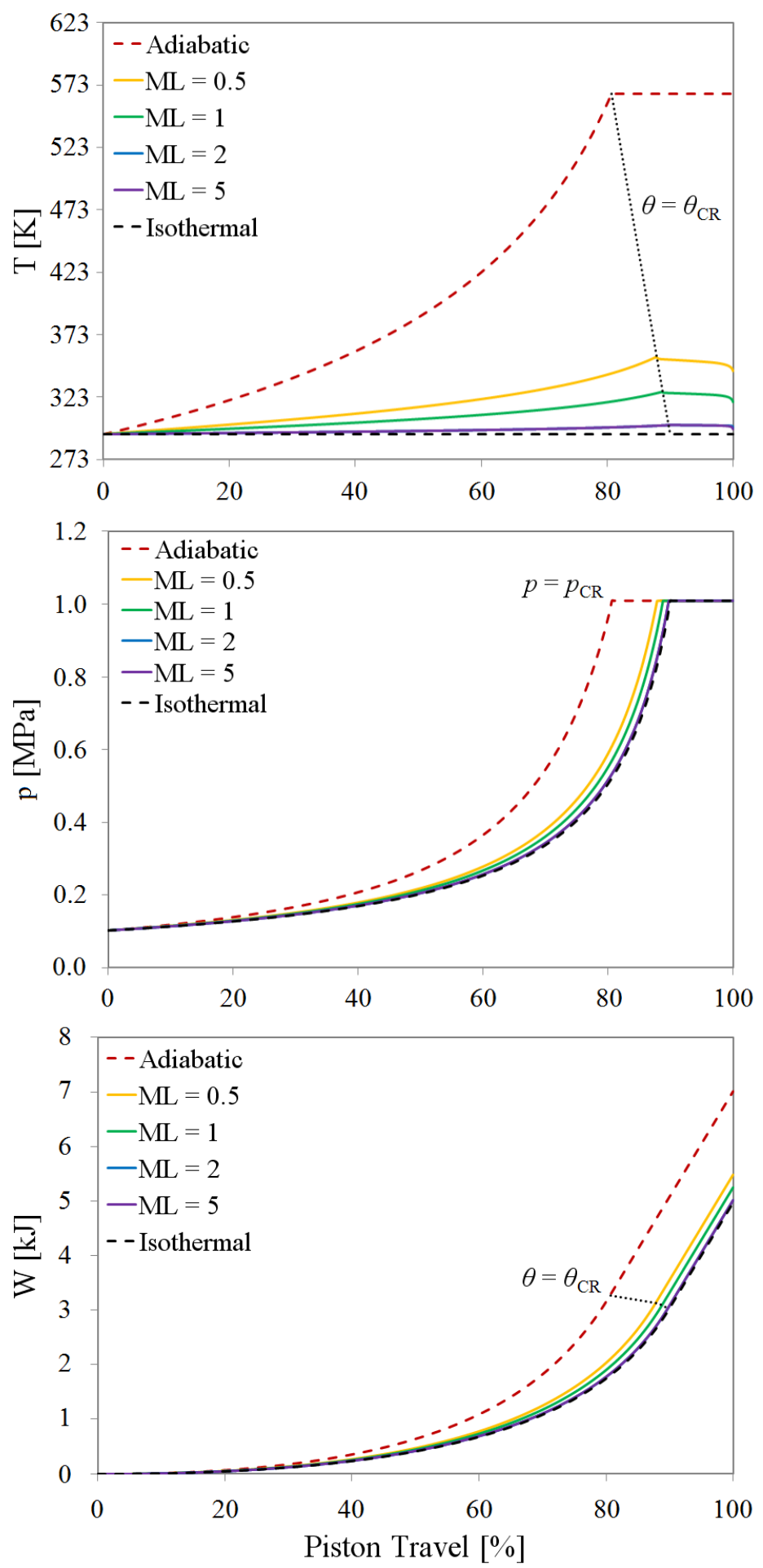

Fig. 2.5 Temperature, Pressure, and Compression Work vs. Piston Travel for Direct Injection $(\mathrm{d}=20 \mu \mathrm{m}, \mathrm{CR}=10)$ 

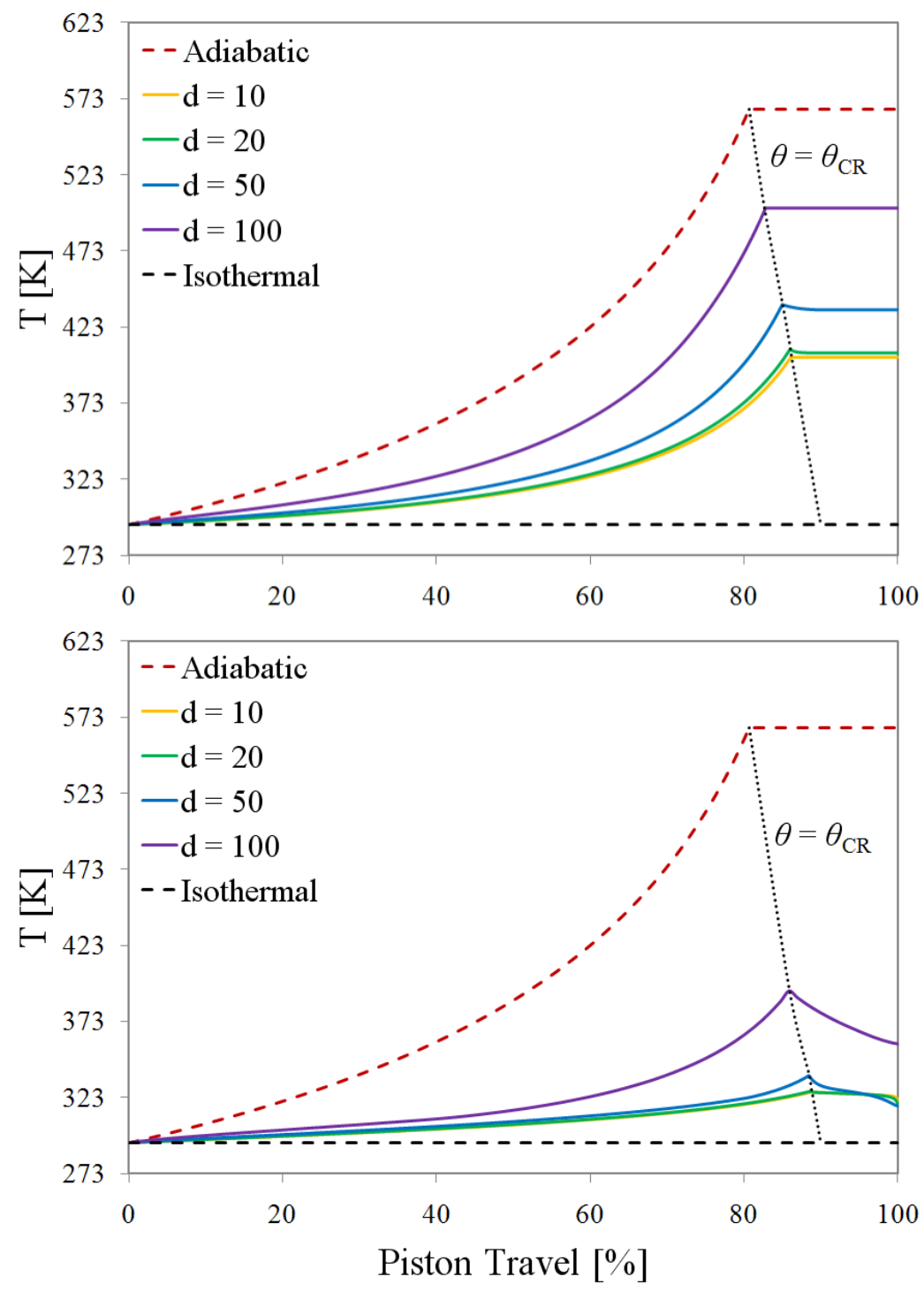

Fig. 2.6 Temperature vs. Piston Travel for $\mathrm{CR}=10, \mathrm{ML}=1$, with droplet diameters of 10,

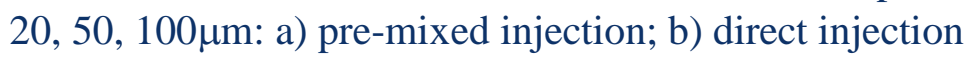

The relationship between the efficiency of liquid piston compression and the total injected surface of droplets is shown in Fig. 2.8 for a pressure compression ratio (CR) of 10. Four curves are shown which describe the direct-injection and pre-mixed cases, where each are also considered for varying mass loading and varying drop diameter. In all cases, increasing the surface area (for fixed injection type, mass loading or droplet diameter) leads to an increase in efficiency. This is especially true when the drop 
diameter is fixed (20 microns) and the mass loading is increased. However, the potential of improving efficiency by increasing heat transfer area is limited in terms of drop size, where it can be seen that an area increase caused by reducing the drop diameter from 20 to 10 microns, does not substantially increase the efficiency, which is consistent with the discussion associated with Fig. 2.6. If one compares the crossing point of the fixed ML curves with the fixed d curves, this similarly shows that increasing mass loading is more effective than decreasing droplet size in terms of improving efficiency. Finally, it can be observed that the direct injection cases demonstrate consistently higher efficiency values than the pre-mixed cases, which is consistent with the discussion associated with Fig. 2.7. These latter two trends are shown more explicitly in Fig. 2.9a, where it can be seen that direct injection with a mass loading of 5 and a drop diameter of 20 microns can lead to efficiencies as high as $98 \%$, which is a dramatic improvement from the adiabatic value of $71 \%$. To examine the effect of pressure compression ratio, the efficiencies for a mass loading of unity (achievable with a single spray nozzle) and a droplet diameter of 20 microns are shown as a function of CR in Fig. 2.9b. The results indicate a nearly linear relationship between the compression ratio and the liquid piston efficiency, whereby an increase compression ratio leads to efficiency decease.

Continued investigations are needed to increase the fidelity of these results. Firstly, the flow dimensionality effects should be addressed with axisymmetric and threedimensional simulations focusing on the key conditions. Secondly, effects of droplet collisions with the wall and each other should be incorporated as this can reduce the surface area of the droplets that are aloft at any given time. Thirdly, practical issues of detailed spray nozzle characteristics, wall heat transfer, liquid piston surface dynamics 
and stability, as well as flow intake and exhaust valving should be considered to determine their impact on both flow and heat transfer physics as well as overall performance. Finally, a multi-stage concept should be analyzed to investigate the suitability and cost-of-energy benefits that the spray-based liquid piston concept can allow for MW-scale energy storage.

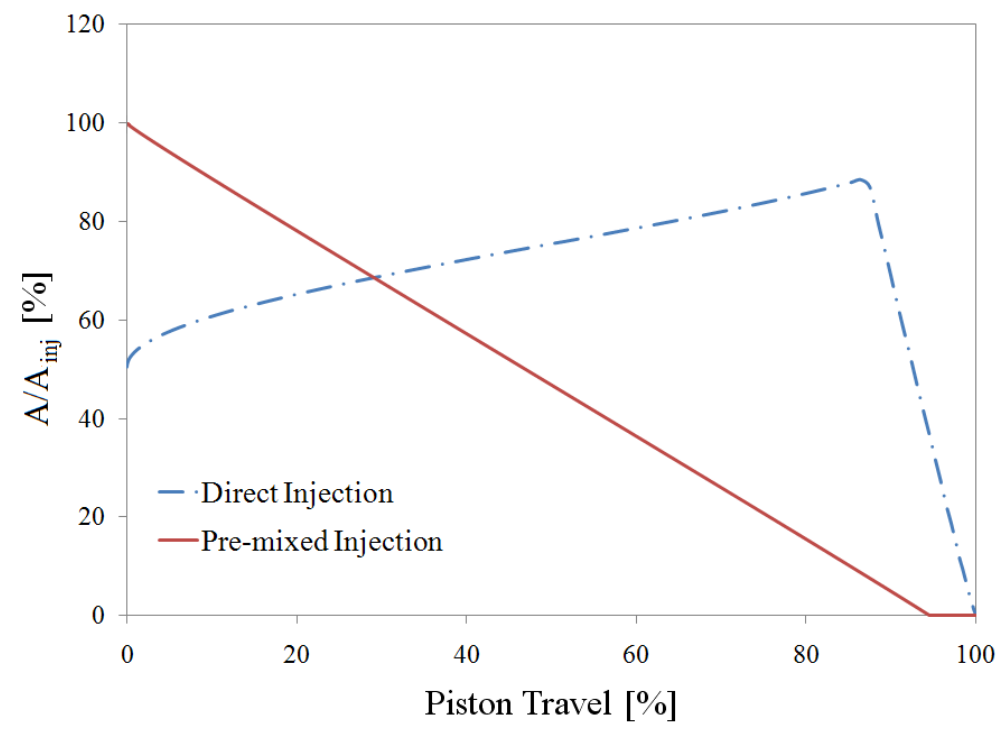

Fig. 2.7 Normalized Droplet Surface Area vs. Piston Travel $(C R=10, d=20 \mu m, M L=1)$

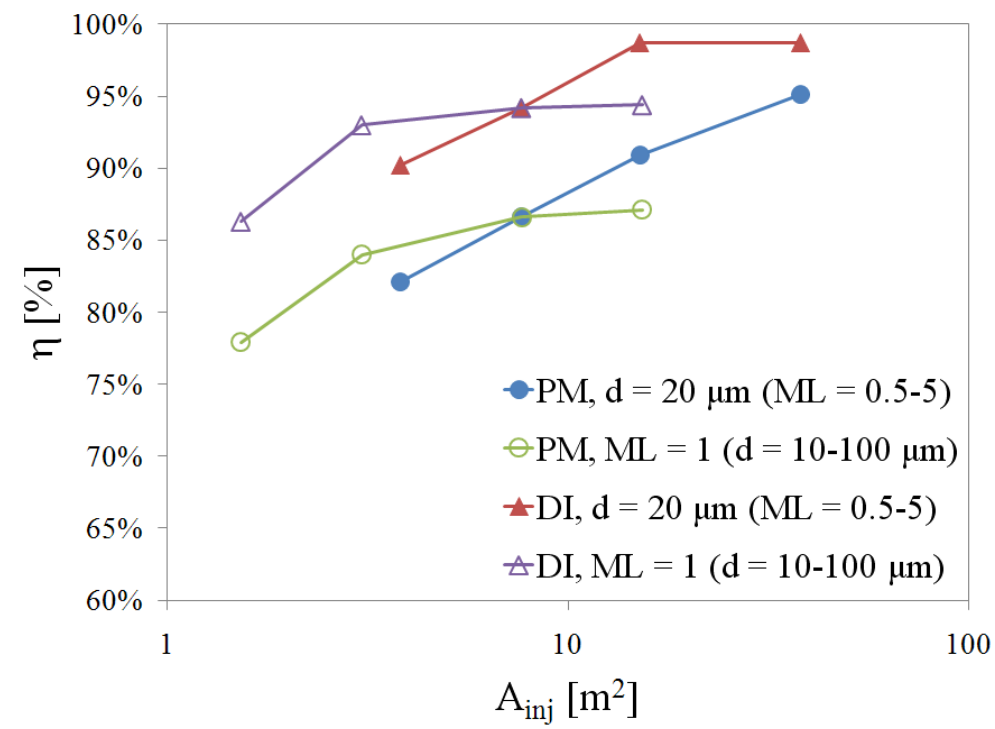

Fig. 2.8 Efficiency vs. Total Droplet Surface Injected $(\mathrm{CR}=10)$ 

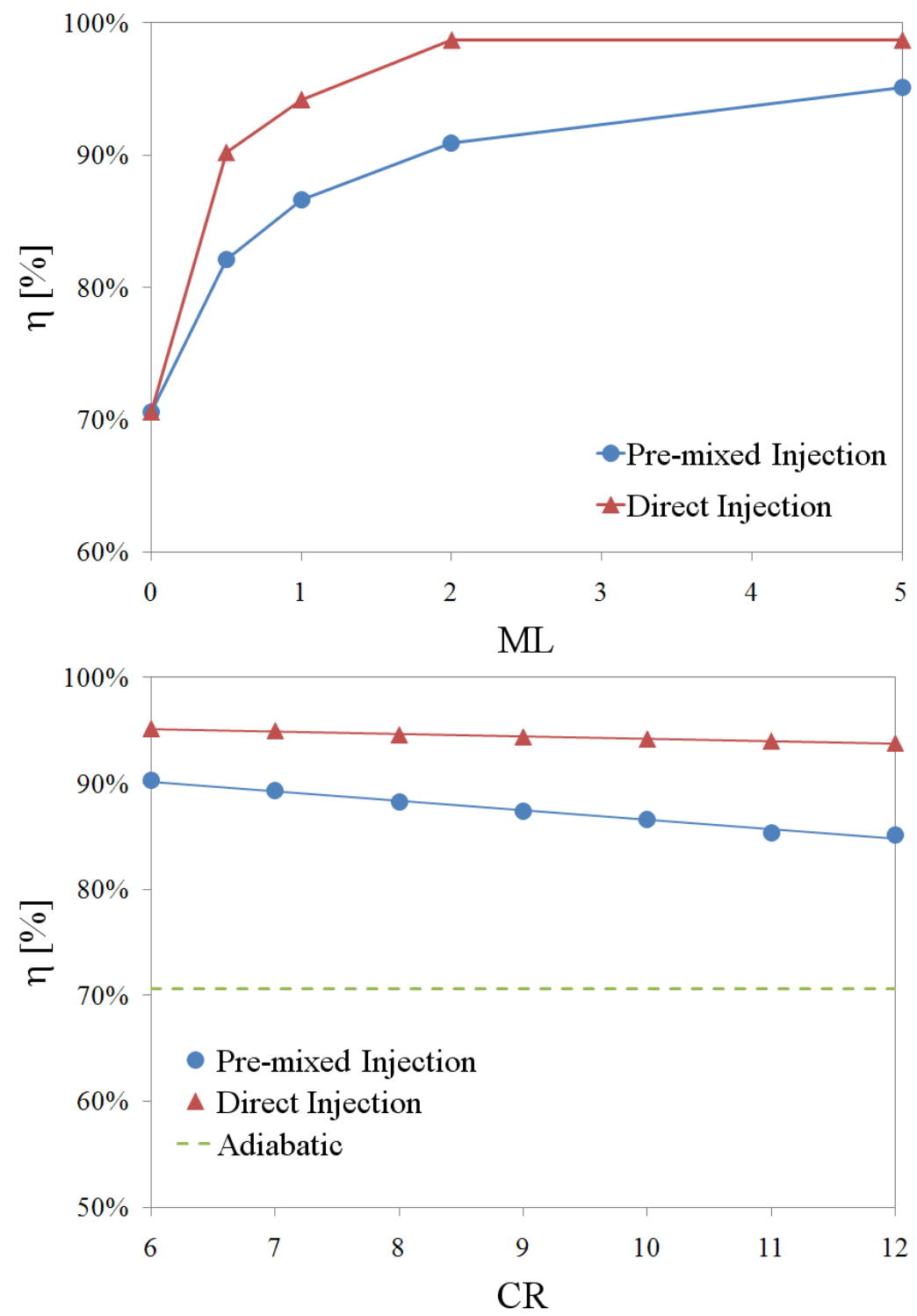

Fig. 2.9 Efficiency a) $\mathrm{CR}=10$ and variable $\mathrm{ML}$; b) $\mathrm{ML}=1$ and variable $\mathrm{CR}(\mathrm{d}=20 \mu \mathrm{m})$ 


\section{Appendix A. Validation of Spray Cooling}

The inter-phase momentum transfer to droplets prescribed by Eqs. 2-6, 2-15 and 2-22 (stemming from Eqs. 2-4a, 2-4d and 2-4f) were used in Ref. 15 and demonstrated excellent agreement with the experimental data in terms of droplet velocity, droplet temperature and droplet evaporation. However, additional validation was desired for the present study to quantify and understand the predictive level of performance with respect to heat transfer on the airflow itself.

For the droplet mass transfer and heat transfer coupling on the gas, a validation was conducted herein based on measurements of water spray evaporative cooling of air ${ }^{[10,11]}$. In the experiments, shown in Fig. A1, water spray is discharged from a nozzle into a horizontal parallel air flow in a duct. As the heat and mass transferred between the air and the droplets, the air is cooled and humidified. Assuming no loss of droplets to the walls, the mass decrease of droplets along the flow is equal to the mass increase or the water vapor fraction in the gas.

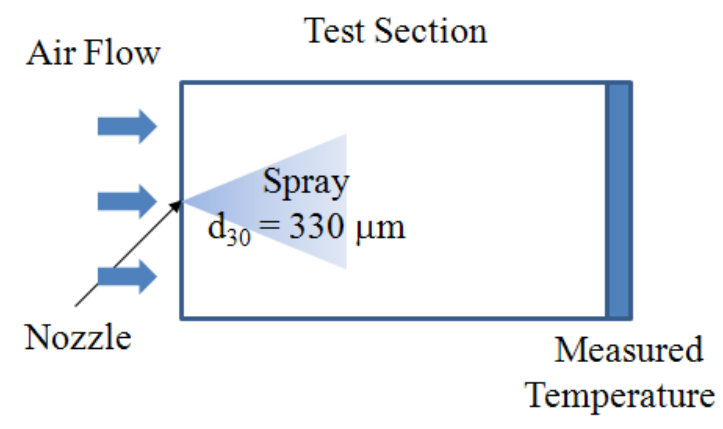

Fig. A1 Schematic of test channel and experimental setting in ITT air-spray system 
To simulate this flow, the Lagrangian formulation of Eqs. 2-5, 2-12, 2-15, 2-19, and 2-22 are converted into a one-dimensional steady Eulerian formulation by re-writing the time derivatives as

$$
\frac{d}{d t}=\frac{d}{d x} \cdot \frac{d x}{d t}=\left\{\begin{array}{lc}
U \frac{d}{d x} & \text { for air } \\
v \frac{d}{d x} & \text { for droplet }
\end{array}\right.
$$

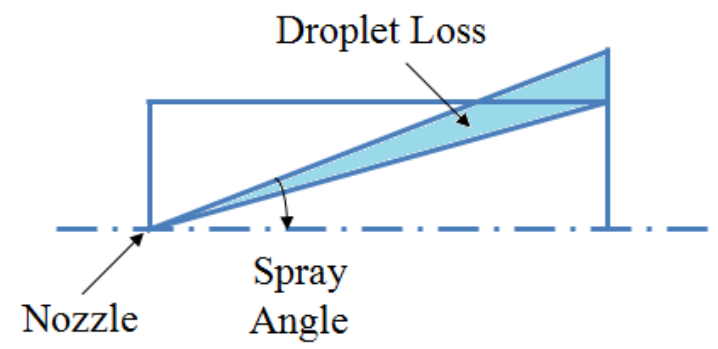

Fig. A2 Schematic of modeling droplet loss

Defining $\mathrm{N}$ as the number of droplets passing across a unit duct cross section area (with constant horizontal gas velocity throughout), six spatial ODEs can be used for the six dependent variables: air density, water vapor mass fraction, droplet diameter, droplet velocity, air temperature, and droplet temperature.

$$
\begin{aligned}
& \frac{d \rho_{g}}{d x}=\frac{N \operatorname{Sh} \rho_{g} \mathcal{D} \pi d\left(\omega_{v, \text { surf }}-\omega_{v}\right)}{U v} \\
& \frac{d \omega_{v}}{d x}=\frac{N \operatorname{Sh} \mathcal{D} \pi d\left(\omega_{v, \text { surf }}-\omega_{v}\right)}{U v}-\frac{\omega_{v}}{\rho_{g}} \frac{d \rho_{g}}{d x}
\end{aligned}
$$




$$
\begin{aligned}
& \frac{d(d)}{d x}=-\frac{2 \operatorname{Sh} \rho_{g} \mathcal{D}\left(\omega_{v, \text { surf }}-\omega_{v}\right)}{\rho_{d} v d\left(1-\omega_{v, \text { surf }}\right)} \\
& \frac{d v}{d x}=-\frac{18 \mu_{g} F(v-U)}{\rho_{d} v d}-\frac{3 v}{d} \frac{d(d)}{d x} \\
& \frac{d T_{g}}{d x}=\frac{h_{v, s} N \operatorname{Sh} \mathcal{D} \pi d\left(\omega_{v, \text { surf }}-\omega_{v}\right)}{c_{p} U v}-\frac{N \mathrm{Nu} k_{g} \pi d\left(T_{g}-T_{d}\right)}{c_{p} U v \rho_{g}}-\frac{h_{g}}{c_{p} \rho_{g}} \frac{d \rho_{g}}{d x} \\
& \frac{d T_{d}}{d x}=\frac{6 \mathrm{Nu} k_{g}\left(T_{g}-T_{d}\right)}{c_{d} \rho_{d} v d^{2}}-\frac{6 L \operatorname{Sh} \rho_{g} \mathcal{D}\left(\omega_{v, \text { surf }}-\omega_{v}\right)}{c_{d} \rho_{d} v d^{2}\left(1-\omega_{v, \text { surf }}\right)}-\frac{3 h_{d}}{c_{d} d} \frac{d(d)}{d x}
\end{aligned}
$$

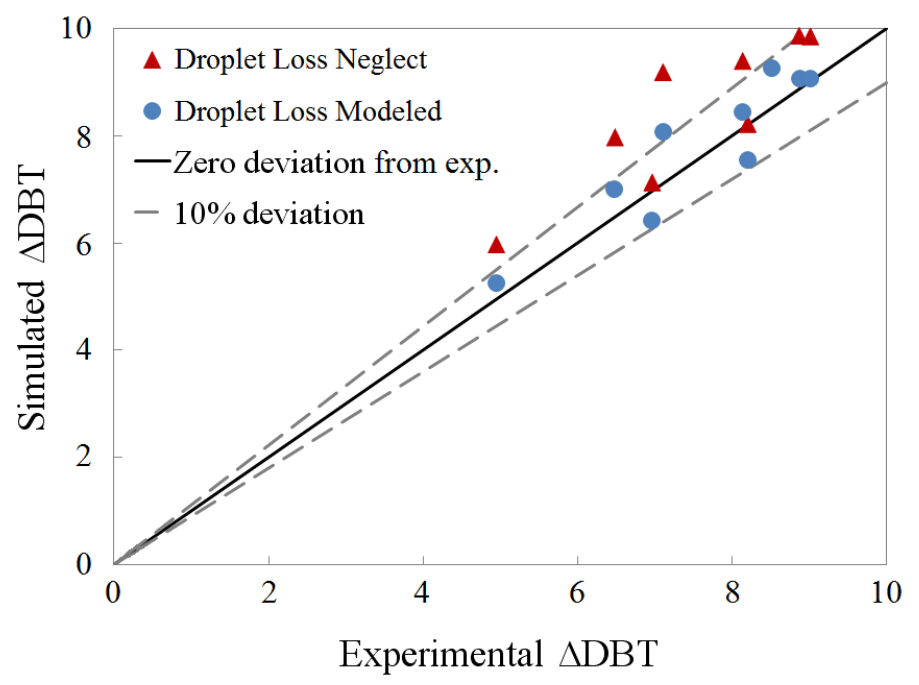

Figure A3 Validation result shown as present simulation deviation from experimental data

This group of six differential equations can be solved using the Runge-Kutta method. The results are shown in Fig. A2 in terms of the dry bulb temperature (DBT) differences between inlet air and outlet air. It can be seen that the simulation results for 
no droplet loss (shown in triangles) show a consistent trend of increasing temperature difference consistent with the experimental values. However, the predicted differences are approximately $20 \%$ larger than experiment data. This discrepancy can be attributed to the loss of some of the droplets lost. As shown by the shaded portion in Fig. A3, a portion of the spray may be expected to intercept the wall within the domain. An estimate for the fraction of droplets lost due to wall impact can be obtained by considering the droplet terminal, the mean velocity vector and the spray cone angle. Assuming further a linear droplet loss in flow direction, the prediction which included this modeled droplet loss is also show in Fig. A2 (as circles) and are within $\pm 10 \%$ of the experimental results, consistent with experimental uncertainty. This indicates that the 1D approach is reasonable for describing heat and mass transfer in a spray-cooling flow. 


\section{Appendix B. Thermodynamic and Psychrometric Properties of}

\section{Humid Air}

Gas in the compression chamber is a mixture of dry air and water vapor, defined herein as "humid air". Since the water vapor and air both act as a perfect gas, an ideal gas equation of state is reasonable. Thus, the pressure in the chamber can be simply obtained in terms of the gas chamber volume $\left(V_{g}\right)$, the gas constant $\left(R_{g}\right)$, the gas mass $\left(m_{g}\right.$, which is constant), and the gas temperature $\left(T_{g}\right)$ as

$$
p V=m_{g} R_{g} T_{g}
$$

The gas density can simply be obtained as the ratio of gas mass to gas volume.

However, the presence of the water vapor can change other properties of the gas that are critical to heat transfer, including viscosity, thermal conductivity, heat of vaporization (so they are not only a function of temperature and pressure, but also of vapor fraction). Previous studies of spray cooling in flowing systems (e.g. as discussed in Appendix A) have reasonably ignored these variations and treated those properties as constant. However, these variations are herein considered because the gas in this study is a closed system subjected to large changes in pressure. The following empirical relations can be used to obtain these properties.

Firstly, the mole and mass fractions are defined. From Dalton's law of partial pressures, the pressure of the humid air is the sum of partial pressures of the dry air and the water vapor

$$
p=p_{a}+p_{v}
$$


The subscripts $a$ and $v$ denote dry air and water vapor components respectively. The mass and mole fraction of water vapor in the humid air can be described in terms of the vapor pressure, specific humidity $(S H)$ and molecular weights of the components $(M)$ as

$$
\begin{aligned}
& x_{v}=\frac{p_{v}}{p} \\
& \omega_{v}=\frac{S H}{1+S H} \quad \text { where } S H=\frac{m_{v}}{m_{a}}=\frac{M_{v}}{M_{a}} \frac{p_{v}}{p-p_{v}}=0.622 \frac{p_{v}}{p-p_{v}}
\end{aligned}
$$

Near the droplet surface, vapor pressure can be approximated by the saturated value, which can be given by Goff-Gratch ${ }^{[26]}$ as

$$
\begin{aligned}
\log p_{v, s a t}= & -7.90298\left(T_{b p} / T-1\right)+5.02808 \log \left(T_{b p} / T\right) \\
& -1.3816 \times 10^{-7}\left[10^{11.344\left(1-T_{b p} / T\right)}-1\right] \\
& +8.1328 \times 10^{-3}\left[10^{-3.49149\left(T_{b p} / T-1\right)}-1\right]+\log p_{b p}^{*}
\end{aligned}
$$

where pressure is given in Pascals and the subscript $b p$ indicates the boiling point at standard atmosphere pressure, i.e. $T_{b p}$ is $373.15 \mathrm{~K}$ and $p_{b p}$ is $101.325 \mathrm{kPa}$. Note that Ref. 26 has a sign error in the above expression that is corrected by Ref. 27.

When the fractions from Eq. B4 are known, the molecular weight and gas constant of humid air can be determined by

$$
\begin{aligned}
& M_{g}=x_{a} M_{a}+x_{v} M_{v}=\left(1-x_{v}\right) M_{a}+x_{v} M_{v} \\
& R_{g}=\frac{R_{m}}{M_{g}}
\end{aligned}
$$


where $R_{\text {univ }}$ is universal gas constant that equals to $8.3145 \mathrm{~J} /(\mathrm{K} \cdot \mathrm{kg}-\mathrm{mol})$.

The mixture (humid air) thermal properties can be calculated based on Wilke and Wassiljewa ${ }^{[28]}$ from the known properties of two individual components (dry gas and water vapor) and their fractions. For specific heat capacity at constant pressure, one may use the definition for ideal gas,

$$
c_{p}=\frac{d h}{d T}
$$

By specifying the enthalpy as a function of temperature, one can use enthalpy changes to obtain an average value of the humid air specific heat capacity. Defining a reference enthalpy of dry air as zero at $0^{\circ} \mathrm{C}$, the humid air enthalpy is given by

$$
h=h_{a}+S H \cdot h_{v}=(1.005 T-274.5)+S H \cdot(1997.4+1.84 T)
$$

where $T$ is the temperature in Celsius, and the units of $h$ are $\mathrm{kJ} /(\mathrm{kg}$ of dry air).

To complete the flow properties, the thermal conductivity, gas viscosity, mass diffusivity and heat of vaporization are needed. For dry air, the viscosity and thermal conductivity of dry air are given by the conventional Sutherland equation and a correlation from Ref. 29. The viscosity and thermal conductivity of water vapor are given by Sengers et al. ${ }^{[30]}$ in terms of temperature and density. The water latent heat of vaporization $L$ can be described using the empirical formulation of Ref. 31. Finally, for mass transfer, the mass diffusivity of water vapor in air is estimated by Bird et al. ${ }^{[32]}$ as

$$
\mathcal{D}=2.495 \times 10^{-5}\left(\frac{T}{292.88}\right)^{2.334} \mathrm{~m}^{2} / \mathrm{s}
$$




\section{Chapter 2 References}

[1] Katz D, Lady E. Compressed Air Storage for Electric Power Generation. Ulrich, Ann Arbor, MI; 1976

[2] Crotogino F, Mohmeyer K, Scharf R. Huntorf CAES: More Than Twenty Years of Successful Operation. Solution Mining Research Institute Meeting Orlando FL; 2002

[3] Davis L, Schainker R. Compressed Air Energy Storage (CAES): Alabama Electric Cooperative McIntosh Plant Overview and Operational History. Electricity Storage Association Meeting: Energy Storage in Action, Knoxville, TN; 2006

[4] Barnes F, Levine J. Large Energy Storage System Handbook. Boca Raton, FL: CRC Press; 2011

[5] Sullivan P, Short W, Blair N. Modeling the benefits of storage technologies to wind power. Houston, TX: American Wind Energy Association (AWEA) Wind Power Conference (NREL/CP-670-43510); Jun. 2008

[6] Greenblatt J, Succar S, Denkenberger D et al. Baseload Wind Energy: Modeling the Competition between Gas Turbines and Compressed Air Energy Storage for Supplemental Generation. Energy Policy 2007; 35: 1474

[7] Salgi G, Lund H. System behavior of compressed-air energy-storage in Denmark with a high penetration of renewable energy sources. Applied Energy 2008; 85: 182-9

[8] Kaldellis JK, Kapsali M, Kavadias KA. Energy balance analysis of wind-based pumped hydro storage systems in remote island electrical networks. Applied Energy 2010; 87: $2427-37$

[9] Pickard W, Hansing N, Shen A. Can Large-Scale Advanced-Adiabatic Compressed Air Energy Storage Be Justified Economically in an Age of Sustainable Energy? J. Renewable Sustainable Energy 2009; 1

[10] Kachhwaha S, Dhar P, Kale S. Experimental studies and numerical simulation of evaporative cooling of air with a water spray - I. horizontal parallel flow. International Journal of Heat Transfer 1998; 41: 447-64

[11] Sureshkumar S, Kale S, Dhar P. Heat and mass transfer processes between a water spray and ambient air - I. experimental data. Applied Thermal Engineering 2008; 28: 349-60 
[12] Sirignano W. Fluid dynamics and transport of droplets and sprays. Cambridge University Press; 1999

[13] Van de Ven J, Li P. Liquid piston gas compression. Applied Energy 2009; 86: 218391

[14] Li P, Van de Ven J, Sancken C. Open accumulator concept for compact fluid power energy storage. In: ASME 2007 International Mechanical Engineering Congress and R\&D Exposition (IMECE2007-42580); 2007

[15] Loth E. Quasi-steady shape and drag of deformable bubbles and drops. International Journal of Multiphase Flow 2008; 34(6): 523-546.

[16] Masters K. Spray drying handbook. 4th ed. New York: Wiley; 1991

[17] Barrow H, Pope CW. Droplet evaporation with reference to the effectiveness of water-mist cooling. Applied Energy 2007; 84: 404-12

[18] Kersey J, Loth E, Lankford D. Effects of evaporating droplet on shock waves. AIAA Journal 2010; 48: 1975-85

[19] Bailey G, Slater I, Eisenklam P. Dynamic equations and solutions for particles undergoing mass transfer. Britain Chemistry Engineering 1970; 15: 912

[20] Ferry J. and Balachandar S. A fast Eulerian method for disperse two-phase flow. Intl. J. of Multiphase Flow 2001; 27: 1199-226

[21] Sazhin S. Advanced Models of Fuel Droplet Heating and Evaporation. Progress in Energy and Combustion Science 2006; 32: 162-214

[22] Hiestand J, Henry J, Farajian S. A simple numerical model of a water spray humifier. In: ISA-paper \#90-11580198-0092/90/1601-1607, 1990

[23] Crowe C, Sharma M, Stock D. The particle-source-in-cell (PSI-CELL) model for gas-droplet flows. ASME Journal of Fluids Energy 1977; 99: 325-52

[24] Sazhin S. Modeling of heating, evaporation and ignition of fuel droplets: combined analytical, asymptotic and numerical analysis. Journal of Physics: Conference Series 22, 2005; 174-193 
[25] Prommersberger K, Maier G, Wittig S. Validation and application of a droplet evaporation model for real aviation fuel. RTO AVT Symposium on "Gas turbine engine combustion, emissions and alternative fuels" in Lisbon 12-16 October 1998

[26] Smithsonian Meteorological Tables. 5th ed. Washington DC: Smithsonian Institution Press; 1984, p. 350

[27] Vomel H. Saturation vapor pressure formulations. CIRES, University of Colorado. See online http://cires.colorado.edu/ voemel/vp.html

[28] Irvine T Jr., Liley P. Steam and gas tables with computer equation. Orlando: Academic Press; 1984

[29] Millat J, Dymond JH, Nieto de Castro CA. Transport properties of fluids: their correlation, prediction and estimation. Cambridge, UK: Cambridge University Press; 1996

[30] Sengers J, Watson J. Improved international formulations for the viscosity and thermal conductivity of water substance. Journal of Physical Chemistry Reference Data 1986; 15: 1291

[31] Torquato S, Stell G. An equation for latent heat of vaporization. Industrial Engineering Chemistry Fundamentals 1982; 21: 202

[32] Bird B, Stewart W, Lightfoot E. Transport phenomena. New York: Wiley; 1960 


\section{Chapter 3 Spray-Cooling Concept for Wind-Based CAES}

\subsection{Introduction}

Current wind turbine systems are conventionally designed based on rated wind speed and peak output power to match the maximum capacity of the electrical generator. However, the wind turbine electrical output is based on the wind conditions rather than the grid energy consumption as shown conceptually in Fig. 3.1. As a result, the peak generated power often does not occur at the same time as peak demand as shown quantitatively in Fig. 3.2. For example, if there is little or no wind, the wind turbine will generally not provide enough electricity supply relative to average or expected consumption. However, the ratio of the average power produced over long times (e.g. one year) to the rated power (maximum possible under rated conditions) is defined as the capacity factor, and is typically $20-40 \%$ for conventional wind turbines. An average capacity factor of $33 \%$ indicates that the generator and transmission lines are over-sized by about three-fold relative to the average power that is generated.

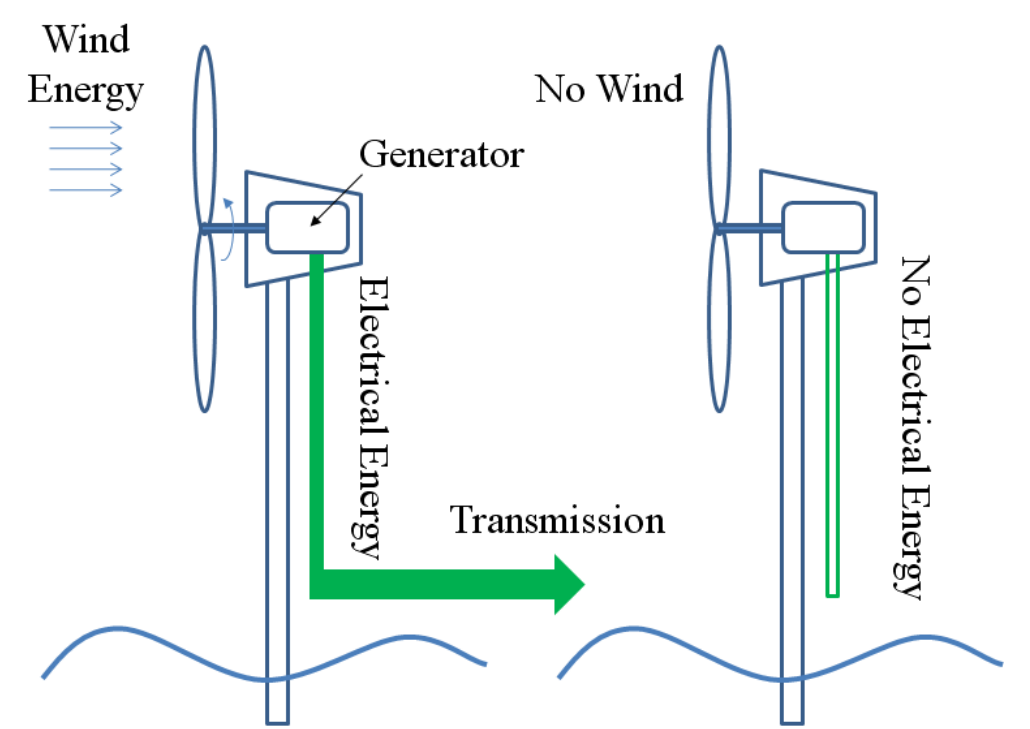

Fig. 3.1 Sketch of conventional offshore wind energy transmission 
This problem of over-sizing generator and transmission lines can be alleviated if efficient energy storage and regeneration technologies are employed so that the net energy from the wind turbine and the storage/regeneration system is "levelized". This storage principle is shown in Fig. 3.2 whereby energy is stored when wind power is larger than the average output, and stored energy is regenerated when the electricity demand is high or available wind energy is low. Current energy storage systems for wind turbines: 1) pumped-hydroelectric storage (PHS) ${ }^{[1,2], 2)}$ batteries ${ }^{[1,2]}$, and 3) compressed-air energy storage $(\mathrm{CAES})^{[1,2,3,4]}$. However, all three of these concepts suffer from shortcomings since: 1) off-shore turbines generally do not have access to elevated reservoirs needed for PHS, 2) batteries are impractically expensive for large-offshore systems, and 3) conventional CAES requires use with natural gas turbines and extremely large volume caverns which are problematic for off-shore turbines. To overcome these barriers, a new CAES concept for wind energy is proposed that is predicted to be both highly efficient and economical while avoiding need for gas turbines or extreme volume reservoirs. This new concept employs a three-stage spray-based open-accumulator compressed air energy system.

To the author's knowledge, no previous published studies have discussed the detailed concept of a spray-based multi-stage compression for energy storage, nor simulated the performance over a range of mass loadings and droplet sizes. In addition, no previous published studies have considered the system characteristics and quantified the benefits of such compressed air energy storage for large-scale off-shore wind turbines in terms of a Levelization Factor. Since these benefits can be significant, the spray-based 
CAES developed and investigated herein is both novel and relevant to future trends in wind energy.

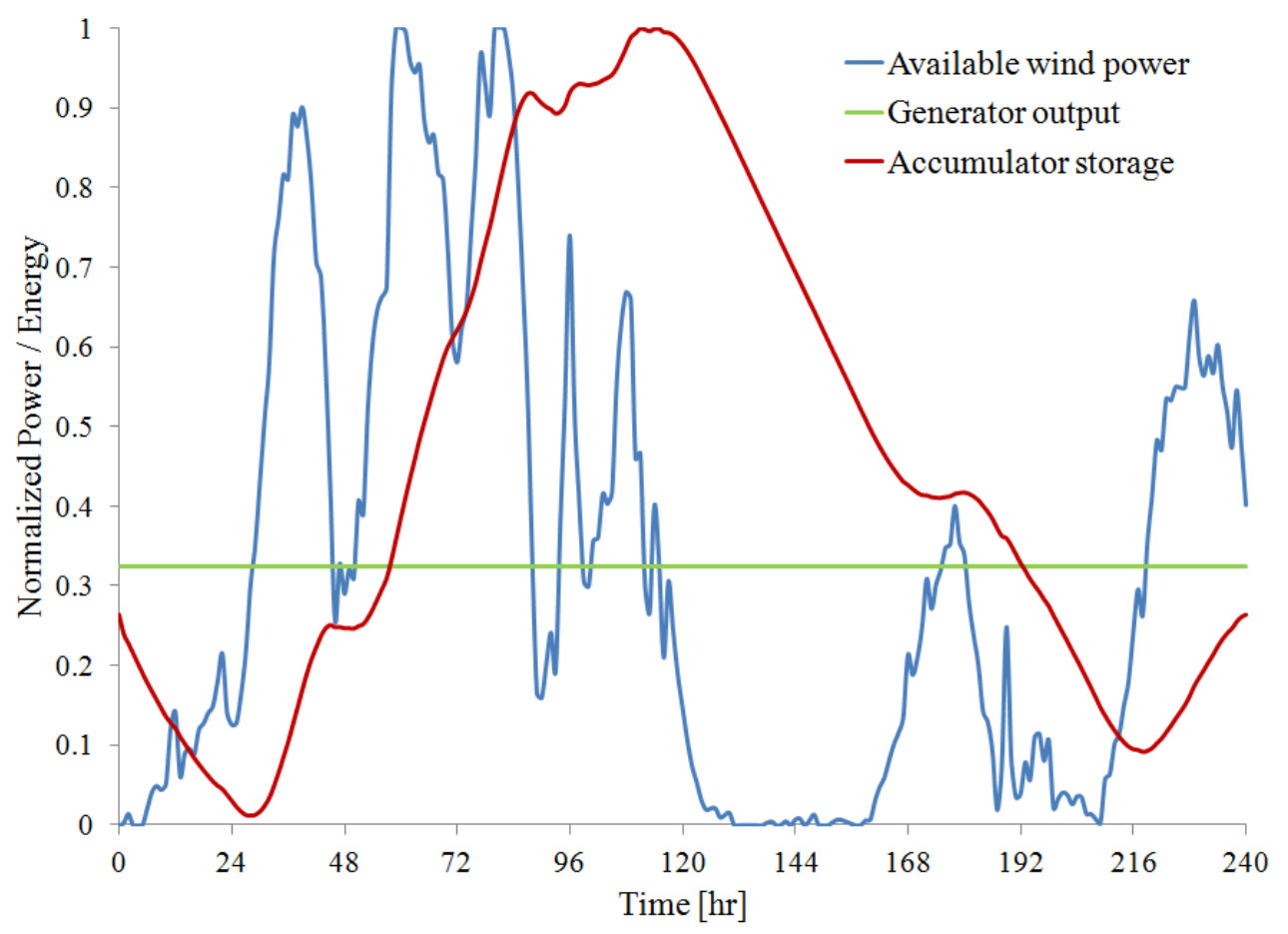

Fig. 3.2 Schematic indicating use of energy storage that provides a constant electrical generator output despite unsteady available wind power

\subsection{Storage Concept, Design, and Analysis}

\subsubsection{Open Accumulator Concept Applied to a Wind Turbine}

A wind energy storage and regeneration system for a multi-MW offshore wind turbine requires consideration of the accumulator design and fluid. Energy storage in a traditional accumulator uses either compressed air or hydraulic energy. In contrast, an “open accumulator" incorporates both compressed gas and liquid, which allows the air pressure to remain high and constant even while energy is extracted ${ }^{[5]}$. This allows high 
storage energy density to be maintained at all time and, importantly, saves both the volume and weight taken by the displaced oil in the traditional closed accumulator. The open accumulator system also does not require site-specific or expensive elevated water reservoirs (as needed for pumped-hydroelectric storage). Furthermore, an open accumulator is much more inexpensive and environmentally friendly as compared to battery storage options.

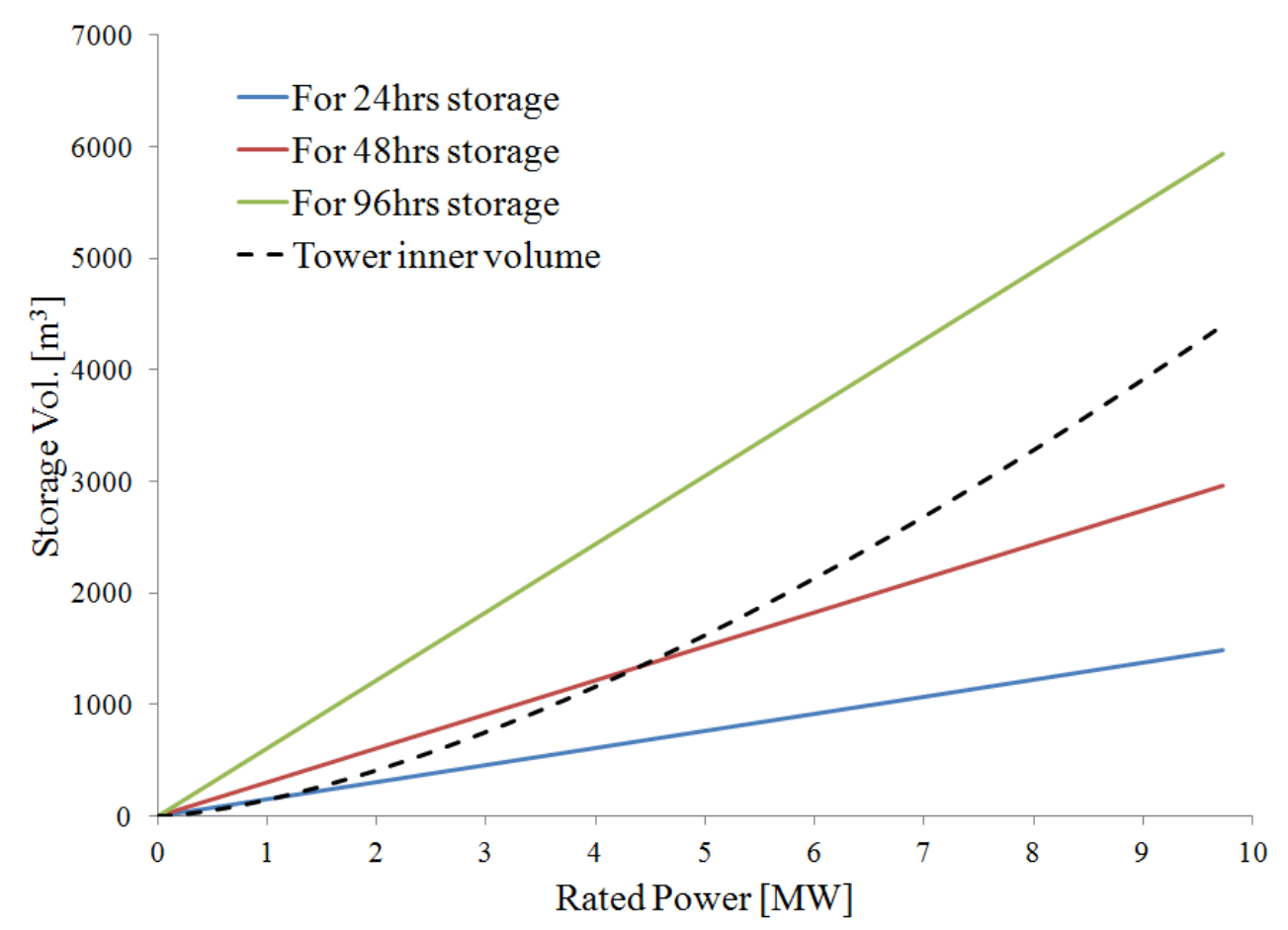

Fig. 3.3 Open accumulator storage volume for one, two and four days worth of energy storage (solid lines) compared the tower inner volume (dashed line) whereby tower height and diameter are assumed to scale linearly with rotor diameter, which is assumed to be proportional to the square root of rated power

The pressure and volume of an open accumulator determines the capacity for storage and regeneration. A typical design may employ a $35 \mathrm{MPa}$ storage pressure vessel, with the design based on a balance between the cost, the container size and safety 
requirements. For this pressure, the storage volume can be computed as a function of wind turbine rated power and days of storage capacity as shown in Fig. 3.3. While the storage volumes are large, they are comparable to the wind turbine tower inner volume for a $5 \mathrm{MW}$ system with 48 hours of storage. This volume equivalence indicates that the storage vessel can be the inner volume of the tower or of an associated floating platform where the storage liquid could serve as ballast to stabilize the system. Thus, large wind turbines are more amenable since the ratio of open accumulator volume to inner tower volume (required for two days of storage) decreases as the rated power increases.

Fig. 3.4 shows the proposed operation of a wind energy storage and regeneration system for a multi-MW offshore wind turbine with an external open accumulator system which was first proposed in Li et al.'s work ${ }^{[6]}$. During storage times (when wind power generation is too high), wind energy is transferred to shaft work by hydraulic pump and motor. Part of the work is used to generate electricity to satisfy the user's demand while the surplus work is stored in the open accumulator through air compression. During regeneration times (when wind power generation is too low), compressed air is allowed to exit the open accumulator and expand near-isothermally to provide power through an air motor that drives the generator to produce electricity. In general, the energy storage and regeneration depend on profile of user's demand and real-time electricity price. Wind energy is stored when it goes higher than the demand, and the stored energy is regenerated and sold at high electricity price when electricity demand is increasing.

Such a concept can be used for a wind turbine to potentially allow a cost-effective and compact means of energy levelization that helps reduce problems associated with supply-demand mismatch that leads to over-sizing of the generator and the transmission 
lines. Another advantage of this concept is that the traditional wind turbine generator on top of the wind turbine tower (Fig. 3.1) is replaced with a hydraulic pump in the nacelle and a fixed-speed electrical generator the base platform (Fig. 3.4). As discussed further in Section 3.3.4, this can help reduce tower costs as well as nacelle-related maintenance costs.
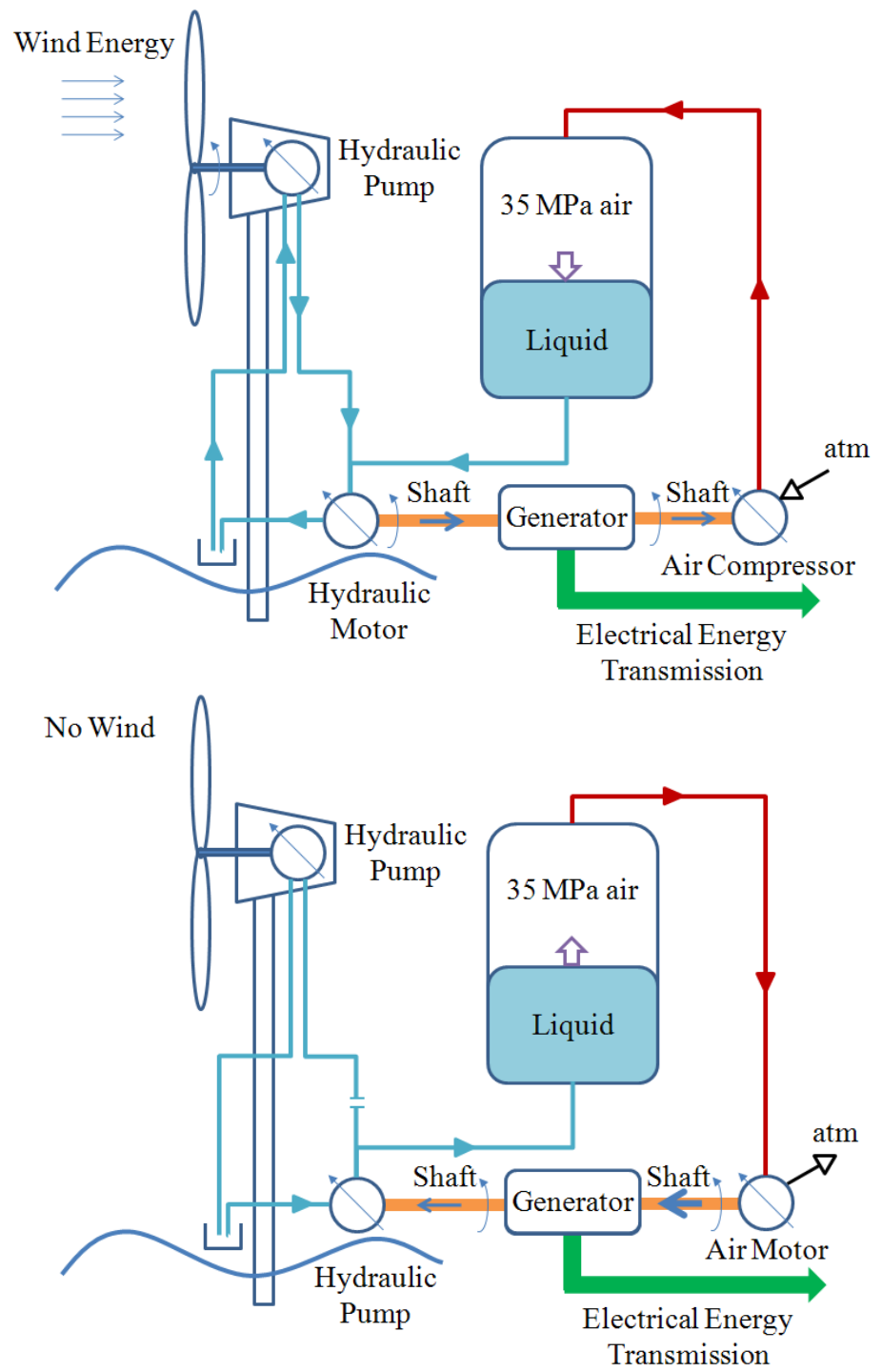

Fig. 3.4 Open accumulator concept operation when: a) energy is both transmitted and stored due to high wind power availability, and b) energy is regenerated due to no wind power availability 


\subsubsection{Spray-Cooling and Multi-Stage Compression}

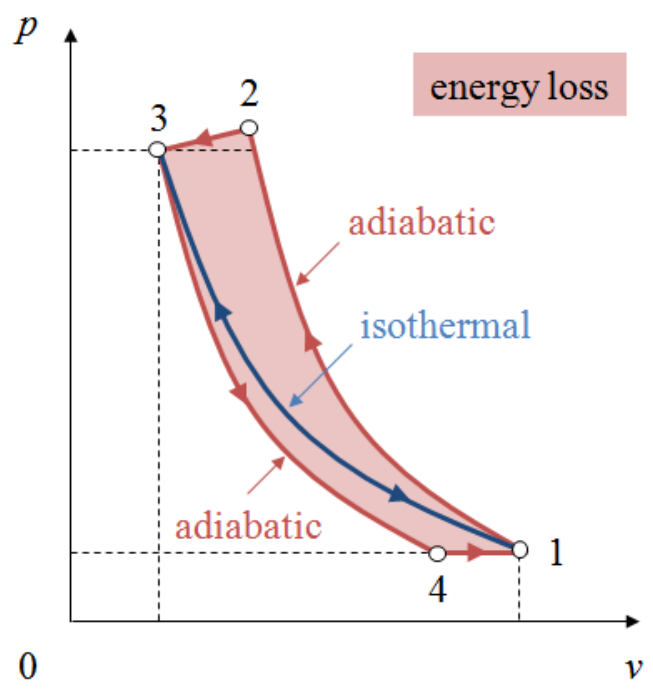

Fig. 3.5 Air pressure as a function of its specific volume comparing an isothermal compression and expansion process (both shown by blue line) and an adiabatic compression and expansion process coupled with non-adiabatic heat rejection to atmosphere while in storage (shown by red line circuit whereby net integral results in energy loss)

For open accumulator application, a high-efficient air compressor/motor is necessary to avoid substantial thermal energy lost after the compression and expansion procedures. As shown in Fig. 3.5, the adiabatic compression process leads to a thermal energy build-up from 1 to 2 . This later dissipates energy from 2 to 3 through heat rejection (without power recovery) to the surrounding atmosphere due to long-time storage in the accumulator. In contrast, an isothermal compressed air energy storage cycle avoids the transfer of compression work to thermal energy (and the associated air temperature increases) so the energy loss is ideally zero. Hence, isothermal compression is more efficient than traditional near-adiabatic compression when considering long-term storage and thermal energy dissipation. Thus, isothermal compression requires less 
mechanical work to compress a given mass of air to a given pressure as compared to near-adiabatic compression since additional work is required to maintain the pressure when the air is cooling down. Note that for a droplet mass loading of unity in the gas, the volume fraction of the spray ranges from $0.1 \%$ to $1 \%$ during compression, so that the droplet volume can be reasonably ignored in $p$-v curve of Figure 3.5.

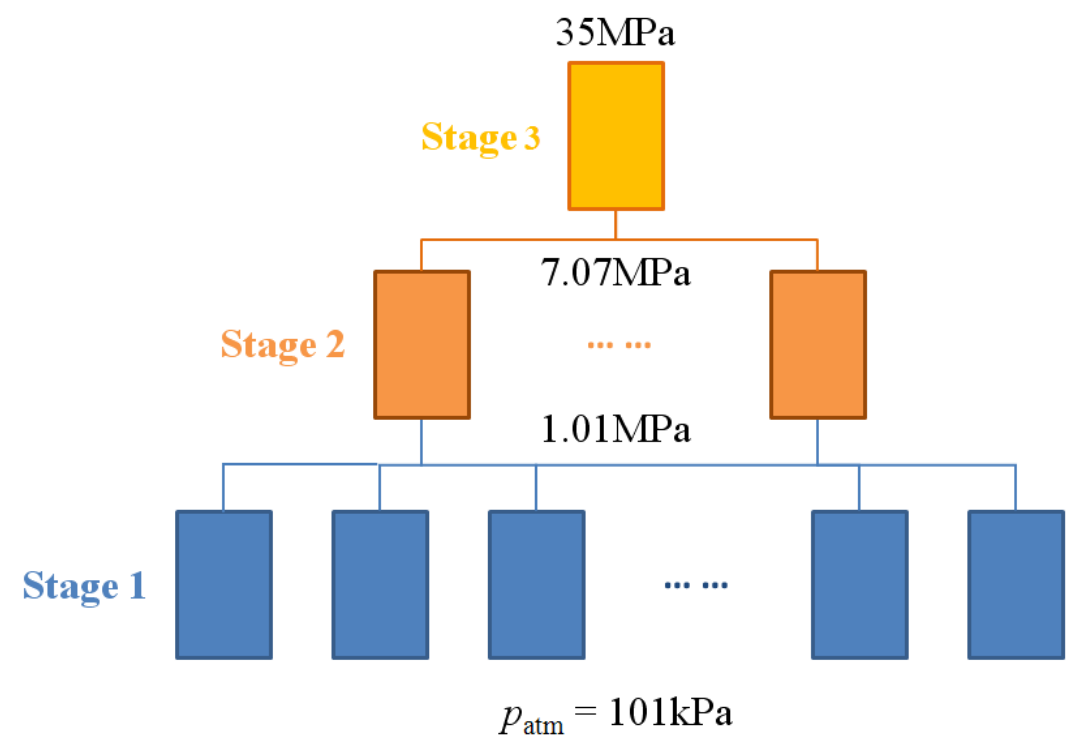

Fig. 3.6 Arrangement of three-stage air compression for offshore wind energy storage assuming constant volume and frequency for the all compression cylinders

To help achieve near-isothermal compression, heat transfer enhancement through water spray-cooling can be used ${ }^{[8,12]}$. Other approaches being investigated include the use of liquid piston through a porous media ${ }^{[13,14]}$ and optimized compression trajectories ${ }^{[10]}$. In this study, the spray-cooling concept is investigated to achieve nearly-isothermal compression for a three-stage compression system, as shown in Fig. 3.6. The compression ratios for the three stages are 10:1, 7:1, and 5:1, based on ratios that are reasonable to achieve in terms of sealing and mechanical operation, and leading to a final 
pressure of $35 \mathrm{MPa}$. For fabrication simplicity, it can be desirable to set the dimensions of the chambers in all three stages to be equal. For each piston cylinder, both the inner diameter and the internal length were set herein as $30 \mathrm{~cm}$ to be consistent with conventional piston compression systems. Since all the piston chambers are of equal size (regardless of stage) and since the air mass for all the cylinders in a single stage is conserved between stages (for steady state operation), the air density entering a given stage is inversely proportional to the number of cylinders in a stage. If the compression is nearly isothermal so that density is nearly linearly proportional to pressure, then the number of piston chambers is reduced from Stage 1 to Stage 3 by a factor of approximately 35. In general, it is expected that spray would employ fresh water (rather than salt water) to mitigate bio-fouling and corrosion effects.

Tab. 3.1 Characteristics of three-stage compression arrangement for a $5 \mathrm{MW}$ offshore wind energy storage system (independent of the degree of spray heat transfer)

\begin{tabular}{cccc}
\hline \hline & Stage 1 & Stage 2 & Stage 3 \\
\hline $\begin{array}{c}\text { Number of cylinders per } \\
\text { stage }\end{array}$ & 210 & 21 & 3 \\
Stage compression ratio & 10 & 7 & 5 \\
Air mass per cylinder [kg] & 0.025 & 0.254 & 1.781 \\
$\begin{array}{c}\text { Energy stored per cylinder } \\
{[\mathrm{kJ}]}\end{array}$ & 4.9 & 41.7 & 241.3 \\
Energy stored per stage $[\mathrm{kJ}]$ & 1029 & 875.7 & 723.9 \\
\hline \hline
\end{tabular}

To determine the stored energy for each cylinder, one may describe the energy density $\left(E_{\eta}\right)$ for an open accumulator system ${ }^{[1]}$ in terms of the compressed air pressure $\left(p_{\text {comp }}\right)$ and surrounding atmosphere pressure $\left(p_{\text {atm }}\right)$ as 


$$
E_{\eta}=p_{c o m p} \ln \frac{p_{c o m p}}{p_{a t m}}-\left(p_{\text {comp }}-p_{\text {atm }}\right)
$$

The stored energy is then the product of energy density and storage volume as listed in Tab. 3.1. Thus, a $3^{\text {rd }}$ (highest pressure) stage cylinder stores much more compressed air energy per cylinder volume than the a $1^{\text {st }}$ (lowest pressure) stage. However, the variation in the number of cylinders per stage and the pressure ratios of Fig. 3.6 allow the overall energy storage per stage to be approximately balanced among all three stages. This balance is a good operational condition for work distribution.

\subsubsection{Liquid Piston Compression with Droplet Heat Transfer}

To help achieve near-isothermal compression, a liquid piston ${ }^{[11]}$ represents a possible solution because it can allow heat transfer enhancement through water spraycooling ${ }^{[8]}$, porous media ${ }^{[9]}$, and optimized compression/expansion trajectories ${ }^{[10]}$. To achieve near isothermal compression, small water droplets can be injected to provide a large surface area for heat transfer between the air and the drops. Both direct and premixed injection concepts were considered by Qin and Loth in prior work ${ }^{[8]}$ for a singlestage system; the results indicated that the direct injection system provided higher overall efficiency for a given amount of water mass injected. This was due to the propensity of direct injection concept to allow more droplets to be aloft, especially during the latter portion of the compression cycle.

As shown in Fig. 3.7, the compression chamber is oriented vertically to keep the entrance/exit ports above the liquid piston. As the liquid piston descends, direct injection initiates water spray with air suction simultaneously so water droplets fill the chamber 
when the suction stroke finishes. As the liquid piston ascends, water spray is also injected during the compression stroke to keep the chamber full of droplets, thereby maximizing heat transfer. When the air pressure reaches the prescribed value of compression, the valve is open to the next stage or the accumulator tank, and the compressed air is pushed out of the compression chamber during the rest of the compression stroke. Given the 30 $\mathrm{cm}$ stroke length, the cycle frequency is set at $1 \mathrm{~Hz}$ so that the maximum acceleration of the liquid interface is limited to be less than half of the gravity acceleration. This generally allows the liquid-gas interface to be stable as the piston is rising and falling.
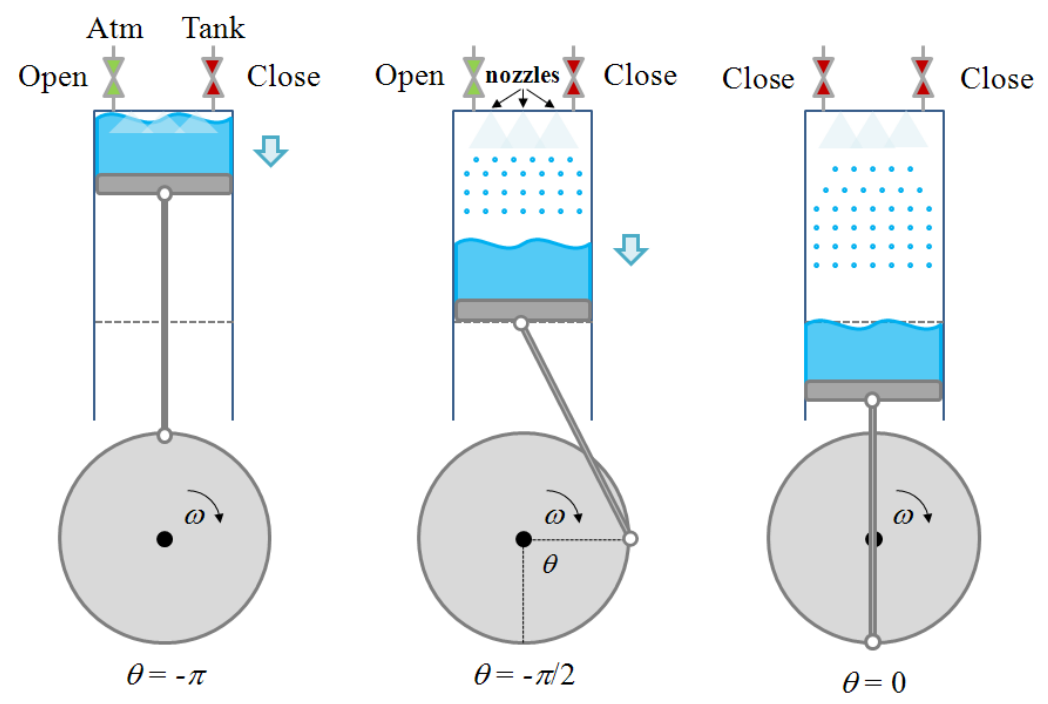

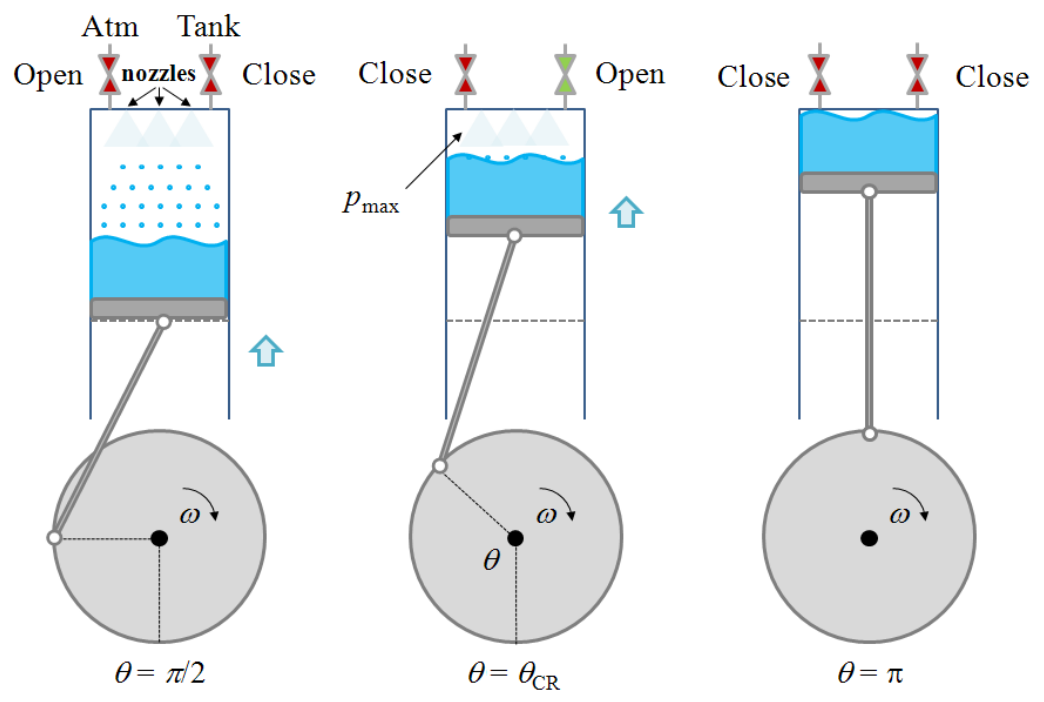

Figure 3.7 Concept of direction injection as a function of stroke angle

\subsubsection{Performance Parameters}

Based on the above concept, there are several parameters that can quantitatively describe the system performance. Foremost among these is the mechanical efficiency that converts the generated power into stored energy through air compression. For this process, the air temperature is ideally held constant due to droplet heat transfer so that the compression is isothermal. However, the overall compression will always be less than ideal due to many practical limitations. The performance under these limitations can be quantified by the compression efficiency for a single cylinder (which is the same as that for the stage) as:

$$
\eta=\frac{\text { Energy stored }}{\text { Compression work + Injection work }}=\frac{E_{\eta} \cdot V_{c o m p}}{\int p d V+\int 2 R T\left[1+\ln \left(\frac{p+\Delta p_{i n j}}{p}\right)\right] d m_{i n j}}
$$


This expression assumes an ideal gas (with gas constant $R$ ) where $p, V$ and $T$ are the cylinder gas pressure, volume and temperature during compression, while $m_{i n j}$ is the water spray mass injected during compression cycle which supplied at an over-pressure of $\Delta p_{i n j}$ (relative to the cylinder pressure). In addition, $V_{\text {comp }}$ is the cylinder volume at the end of the compression cycle.

The three most important spray factors influencing this efficiency are: spray injection work, liquid flow rate, and mean drop size. Spray injection work, which is the work applied to inject droplets into the compression chamber through the nozzles, affects the efficiency since most of this mechanical energy cannot be recovered. The second two factors determine the quantity of spray heat transfer, because large liquid flow rate and small drop size can help to enhance heat transfer and reduce the compression work ${ }^{[8]}$. As such, the spray systems should take into account all three factors to maximize compression efficiency.

Two other parameters that can be used to quantify the system performance are the capacity factor (as conventionally defined) and the levelization factor (a new parameter defined in this study). The capacity factor of a wind turbine is generally defined as

$$
\mathrm{CF} \equiv \frac{\text { Average electrical energy output }}{\text { Maximum electrical energy output at rated rotor power }}
$$

As an example, the present simulations are based on a $5 \mathrm{MW}$ offshore wind turbine whose average annual power is assumed to be $1.75 \mathrm{MW}$ based on a capacity factor of $35 \%$. This is a reasonable assumption since wind turbine capacity factors tend to range from $20 \%$ to $40 \%$ and larger off-shore turbines tend to be higher, on average. If 
a storage system is integrated with such a wind turbine, the degree of generator size reduction possible can be quantified by a Levelization Factor defined herein as

$$
\mathrm{LF} \equiv \frac{\text { Average electrical energy output }}{\text { Peak electrical energy output of generator }}
$$

For conventional wind turbine system, the levelization factor simply equals the capacity factor since its rated generator power is the same as its rated rotor power. As such, the generator is typically over-sized by three-fold to account for wind variations in time. However, a compressed air energy storage system can dramatically increase the levelization factor (to approach unity) by introducing a sufficiently large accumulator. Such a storage concept results in a much smaller generator (working under a constant rpm at a continuous energy output) and smaller associated transmission. However, the overall storage and regeneration efficiency will tend to result in a lower capacity factor and this aspect should be carefully considered.

In the following section, the above performance parameters will be investigated with respect to direct injection spray-cooling for a compressed air energy system coupled to a $5 \mathrm{MW}$ wind turbine. The physics equations and the numerical method for this multiphase unsteady analysis are described in prior work ${ }^{[8]}$ including detailed validation with experimental data of spray-cooled air flow. For the present chapter, the droplets are injected from the top of the chamber with a velocity corresponding to the liquid injection flow rate. The droplet positions and velocities are then updated based on the equation of motion using drag and gravity forces. Since the humidity levels are very high (near $100 \%$ ) and the droplets are cold relative to the gas around, the droplets perform as condensers. Thus, mass transfer effect is minimal and it can be assumed to be negligible. 
To quantify the heat transfer, the liquid and gas thermal properties are also characterized. In particular, the droplets are injected at ambient air temperature and are then updated based on heat transfer relations using the relative temperature difference between the drops and surrounding air. The numerical integration of the temperature evolution for both the air and the droplets is based on a two-step approach. In the first step, the gas is compressed adiabatically based on the volume change associated with piston movement. In the second step, heat transfer between the droplets and the gas is computed based on the temperature difference, to determine the temperature of the air and the drops. After these two steps, the ideal gas law is employed to obtain the new gas pressure. The timestep integration is repeated until the pressure reaches the desired maximum value, after which the exhaust valve is opened. Once this valve is opened, the pressure is assumed to be constant while the piston pushes the compressed gas into the

next stage of the accumulator. As described in previous study ${ }^{[8]}$, this allowed high temporal accuracy and numerical stability despite the small thermal response times of the droplets.

\subsection{System Performance Results}

\subsubsection{Liquid Piston}

Based on the three-stage, $35 \mathrm{MPa}$ storage system of Fig. 3.6 and the direct injection spray-cooling process of Fig. 3.7, the energy storage was analyzed for both adiabatic compression (no spray-cooling) and spray-enhanced compression. The spraycooling was initially investigated at a mass loading (mass ratio of water injected to air in the chamber) of unity, assuming the spray is characterized by 30 micron drops from pressure swirl nozzles. For these conditions, the predicted overall work and temperature 
increases are described in Fig. 3.8 for both adiabatic compression (no spray-cooling) and spray-enhanced compression. In both cases, the same amount of mass is compressed to the same overall final pressure, i.e. the storage energy after all three stages is the same regardless of whether spray is injected. As such, the increased work requirements demonstrated in Fig. 3.8a for the adiabatic compression reflects a reduced efficiency as compared to the spray-based results. This increased work is associated with the higher temperature obtained for the adiabatic case as shown in Fig. 3.8b. This increased thermal energy built up in a few seconds will be generally lost when the air is placed in the open accumulator for several hours or days as the heat is rejected to the atmosphere. 

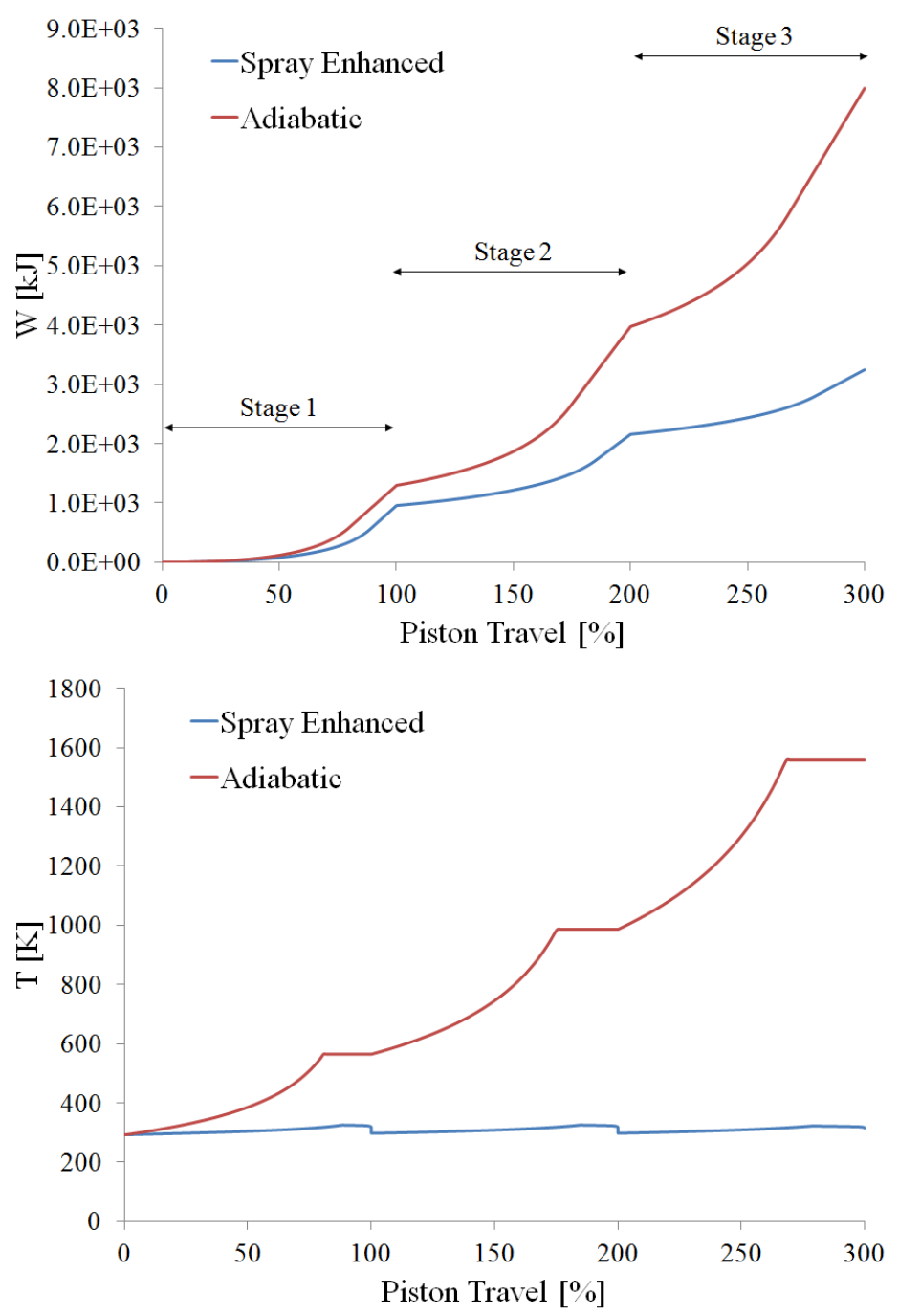

Fig. 3.8 Compression characteristics as a function of piston travel for all three stages for a fixed final compression ratio: a) compression work; and b) temperature increase

Based on the above results and Eq. 3-2, the total compressive efficiency of this three-stage liquid piston with spray-cooling is $89.0 \%$ while the adiabatic version is $26.7 \%$. Table 3.2 shows the individual efficiency for each of the three stages for the spray-based case. The overall efficiency decreases as the stage pressure increases because the compression work per stage increases yielding higher power densities which thus require 
higher heat transfer rates. In addition, the total spray injection work increases since the nozzles must operate in higher gas pressure systems. A further complication of the $3^{\text {rd }}$ stage is that high air density combined with a mass loading of unity yields 15 nozzles per cylinder (Tab. 3.2). This large number will require specialized arrangement if they are all to be located near the valve ports. As such, heat transfer by porous media (instead of spray-cooling) may be more practical for the third stage. This result indicates that a more detailed assessment of the spray characteristics is important for design, since droplets impinging on walls may accumulate and be lost with respect to proving heat transfer. However, it is worth noting that if the nozzles had to be doubled because half of droplets were lost due to wall interactions, the reduction in compression efficiency would be less than $1.5 \%$ based on the above analysis.

Tab. 3.2 Performance characteristics of the proposed spray heat transfer three-stage compression design as described by Fig. 3.6 and Tab. 3.1

\begin{tabular}{cccc}
\hline & Stage 1 & Stage 2 & Stage 3 \\
\hline Num. of nozzles & 1 & 5 & 15 \\
Injection work per cylinder & 0.0714 & 0.713 & 4.97 \\
{$[\mathrm{~kJ}]$} & & & \\
Injection work per stage [kJ] & 15.0 & 15.0 & 14.9 \\
Compression work [kJ] & 1090 & 961.8 & 832.1 \\
Efficiency [\%] & 93.1 & 89.6 & 85.5 \\
\hline \hline
\end{tabular}

\subsubsection{Influence of Spray Characteristics}

To maximize droplet surface area for a spray injector, small drop size and large injection flow rates during the compression period are required. The drop size for a polydisperse spray is most commonly quantified by the Sauter mean diameter (SMD) 
defined as the diameter of a sphere that has the same volume/surface area ratio as that when averaged over the entire drop distribution. This ratio is important as it is proportional to the ratio of droplet thermal inertia to heat transfer rate. The experimental relationship between water flow rate and droplet SMD for several spray nozzles ${ }^{[15-17]}$ at atmospheric pressure are shown in Fig. 3.9. These measurements indicate that it is not easy to simultaneously achieve large liquid flow rate and small drop size by a single nozzle. For example, the Mod-1 and Standard NASA nozzles ${ }^{[16]}$ can produce tiny droplets $(\mathrm{SMD}<20 \mu \mathrm{m})$ but the water flow rate is relatively small. In contrast, the nozzles of Inamura \& Nagai have large flow rate $(>1 \mathrm{~L} / \mathrm{min})$ but the size of the drops are significantly larger. Another issue that must be considered is that both of these nozzles rely on air injection for atomization. This aspect can be quantified in terms of the volumetric gas liquid ratio (GLR), which is defined as the cubic meters of gas injected per liter of water injected. Figure 10 quantifies the gas atomization injection effects on the liquid piston performance for all three stages. It can be seen that increased gas injection rapidly reduces the efficiency of liquid piston especially for the high-pressure $3^{\text {rd }}$ stage. This is because of the work required to inject the high-pressure air (the second term in the denominator of Eq. 2-2). Even for the $1^{\text {st }}$ stage, injection with GLR = 1 causes about a $10 \%$ decrease in efficiency as compared to GLR $=0$. Thus, nozzles with no gas injection like the Wang and Lefebvre swirl atomizer nozzles ${ }^{[15]}$ will generally ensure the highest efficiency. Based on Fig. 3.9, a single such nozzle (shown by green triangles) operating at the most favorable conditions for high droplet surface area can produce $30-\mu \mathrm{m}$ droplets at a relatively large flow rate of $0.72 \mathrm{~L} / \mathrm{min}$. Based on a mass 
loading of unity for all three stages, the number of swirl atomizer nozzles per piston must increase for the higher stages as shown in Tab. 3.2.

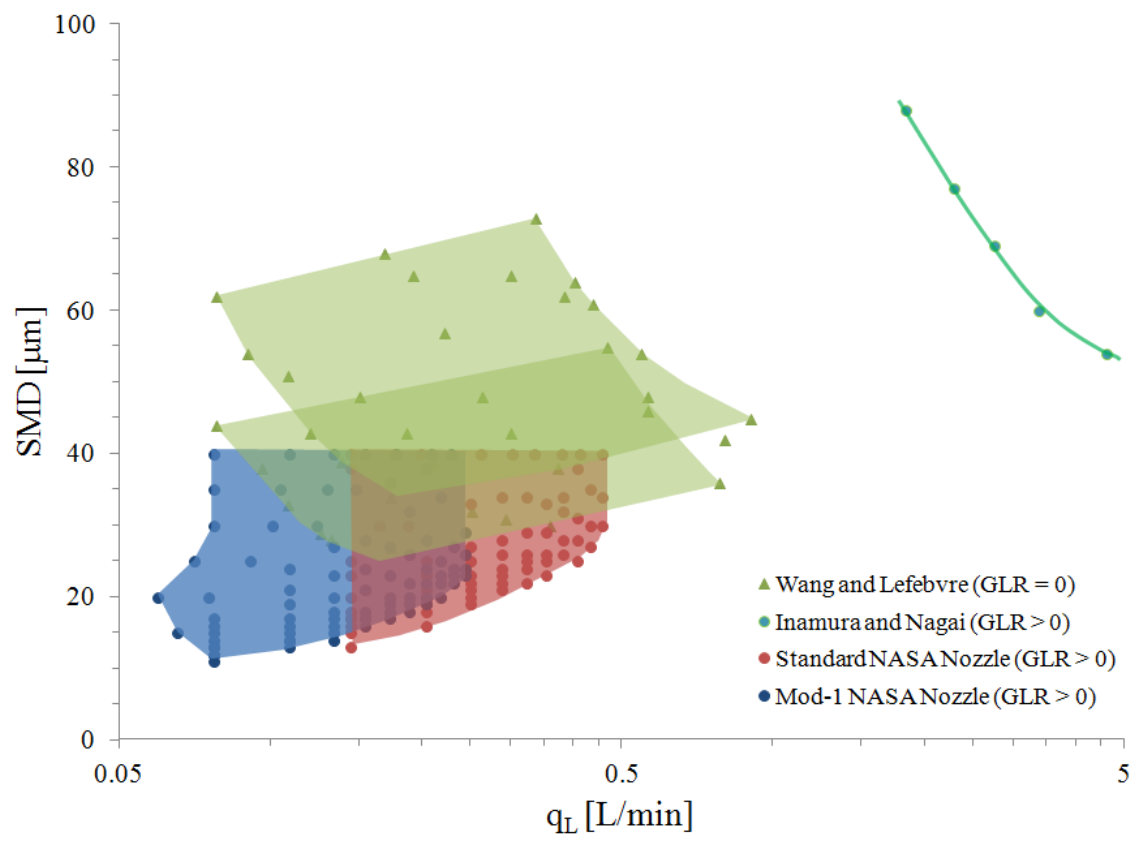

Fig. 3.9 Experimental performance of various single nozzles in terms of liquid flow rate and SMD, where air injection nozzles are denoted with circle symbols (from data available in Ref. 16 and 17), whereas liquid-flow only nozzles are denoted with triangular symbols (from data available in Ref. 15) 


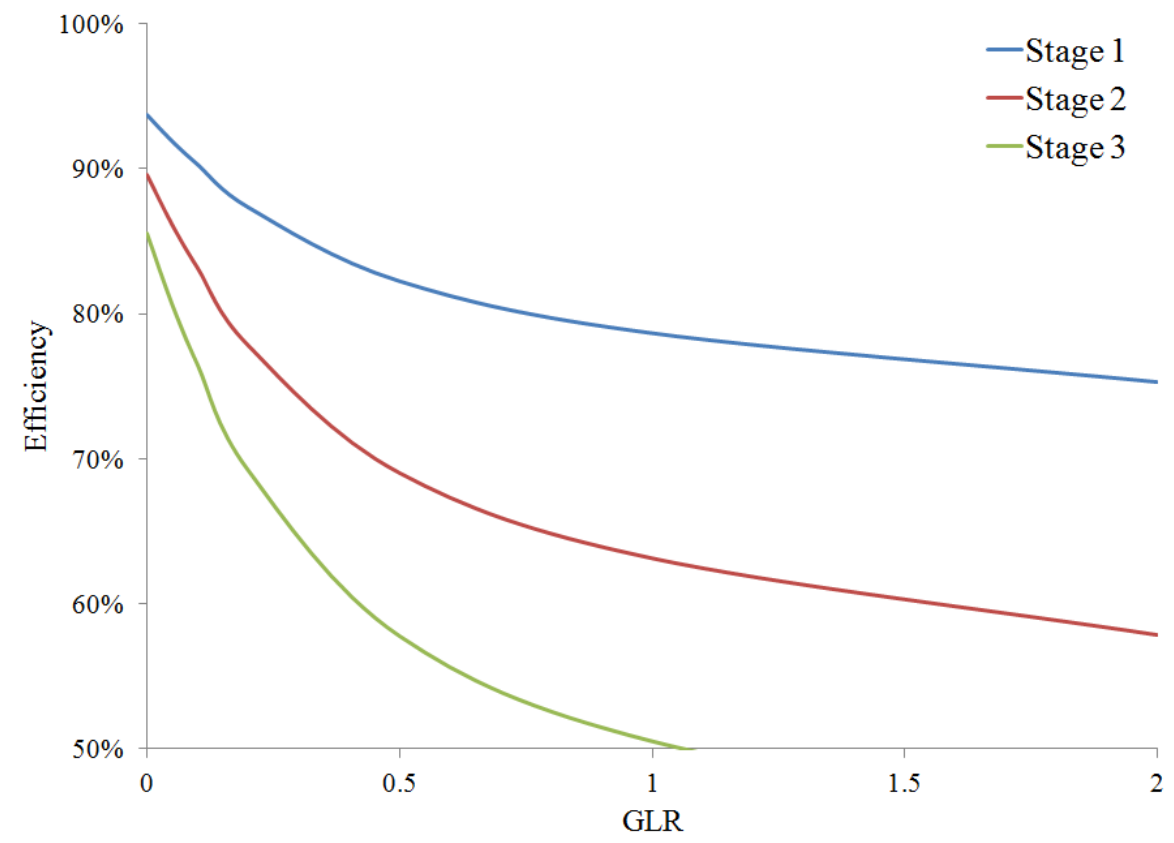

Fig. 3.10 Gas liquid ratio (GLR) impact on liquid piston efficiency for various stages for a spray droplet size of $30 \mu \mathrm{m}$ and a spray mass loading of unity

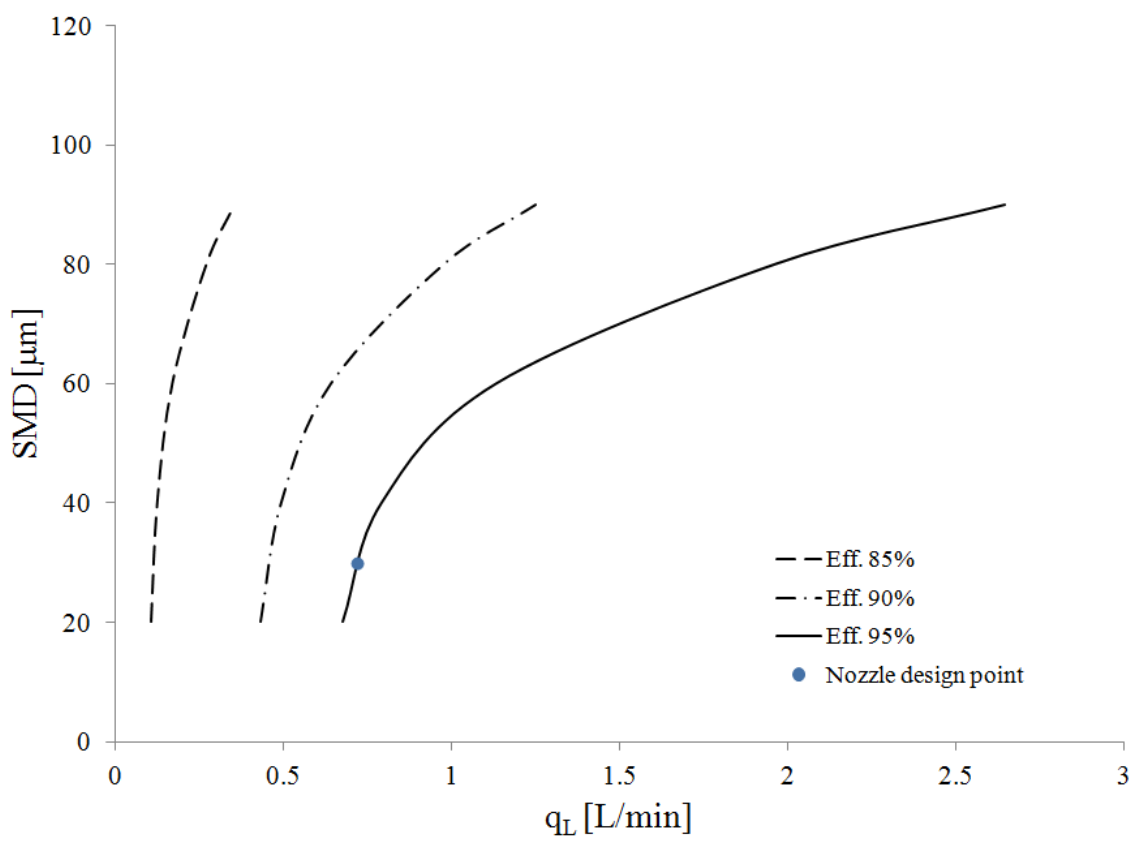

Fig. 3.11 Compressive efficiency as function of liquid flow rate and spray drop size for first stage and a reasonably achievable nozzle design point that results in $95 \%$ efficiency 
Figure 3.11 quantifies the joint impact of droplet size and liquid flow rate for the cooling spray on the first stage compression efficiency for direct injection. In general, the combination of a high spray discharge flow rate and a small droplet size provide high efficiency. However, for the cycle conditions considered herein the benefits are small for droplet sizes less than $20 \mu \mathrm{m}$ and that at least $0.6 \mathrm{~L} / \mathrm{min}$ is needed for $95 \%$ efficiency. Importantly, this figure also shows that a 95\% compressive efficiency can be achieved with a single Lefebvre pressure swirl nozzle producing $30 \mu \mathrm{m}$ droplets at a flow rate of $0.72 \mathrm{~L} / \mathrm{min}$. This nozzle design point in Fig. 3.11 is noted to be experimentally realizable based on the right-most edge of the experimental data for this nozzle in Fig. 3.9. Thus, high efficiency can be obtained with a single nozzle per piston cylinder for the first stage, which corresponds to a mass loading of near unity. However, the increased air density for the high-pressure stages will result in more air mass per piston; thus, more water injection is required per piston to maintain similar mass loadings. As such, more nozzles per piston are required for Stage 2 and 3, as shown by the first row of Tab. 3.2.

\subsubsection{Impact on Generator Size and Levelization Factor}

For transient system performance, CAES system for off-shore wind energy has been recently investigated in terms of the control system ${ }^{[22]}$. However, it is important to quantify the overall impact of energy storage on the system performance parameters as this most directly affects the cost of energy. It is important to note that there are also efficiency losses due to the addition of the hydraulic system (even when there is no storage occurring) and due to the air compressor and expander (during storage and then regeneration). To quantify the capacity and levelization factors as defined in Section 3.2.4, the statistical wind power distribution over time must be estimated. To make the 
analysis straightforward and fundamental, this continuous probability distribution is simplified into three discrete bins corresponding to wind speeds at: full, partial, and zero loads. These three bins can be quantified in terms of probability of occurrence by $x_{1}, x_{2}$ and $x_{3}$ where $x$ is the load frequency distribution and 1,2,3 indicates the condition of full loads, partial loads, and zero loads. In the conventional system (without storage or a hydraulic pump/motor), the overall output simply becomes $P_{\text {out }}=\eta_{\mathrm{G} 1} P_{1} x_{1}+\eta_{\mathrm{G} 2} P_{2} x_{2}$ where $\eta_{\mathrm{G} 1}$ corresponds to generator efficiency at rated conditions (with constant rpm) and $\eta_{\mathrm{G} 2}$ corresponds to generator efficiency at partial load conditions (with variable rpm, leading to lower efficiency). In the CAES application, the power output can then be modeled as a function of the efficiencies for all three loads as

$$
P_{o u t}=\frac{\eta_{G}\left(P_{1} \eta_{H P 1} x_{1}+P_{2} \eta_{H P 2} x_{2}\right)}{\frac{x_{3}}{\eta_{L P}^{2}}+\left(x_{1}+x_{2}\right)}
$$

In this expression, the efficiency subscripts G, HP, and LP indicate generator, hydraulic pump, and liquid piston, respectively. Note that the generator efficiency is taken as $\eta_{G 1}$ in Eq. 5 since the storage concept allows the generator to always operate in fixed rpm conditions.

To estimate available power in each of the above three bins, data from 1993-1999 along the German coastline and islands ${ }^{[18]}$ is used, with an average wind power of 176 $\mathrm{W} / \mathrm{m}^{2}$ and a $15 \%$ deviation. This information is translated to a prototypical wind turbine that has a rotor diameter of $124 \mathrm{~m}$ (consistent with the UK $5 \mathrm{MW}$ wind turbines ${ }^{[19]}$ ) so that the annual average available wind energy is $2.125 \mathrm{MW}$ (for each $5 \mathrm{MW}$ rated turbine). Based on data from Germany and California coastal locations ${ }^{[18,20]}$, the average 
annual times spent in full load $\left(x_{1}\right)$ is $26 \%$, and in partial load $\left(x_{2}\right)$ is $59 \%$, and in no load $\left(x_{3}\right)$ is $15 \%$. However, converting this wind energy is into rotor shaft mechanical energy and then into delivered electrical energy is associated with losses (based on the rotor aerodynamic efficiency and the power efficiency). Herein, the combination of losses for the conventional system is estimated to yield a net overall efficiency of $90 \%$ at full load $\left(\eta_{\mathrm{G} 1}\right)$ and $70 \%$ at the partial load $\left(\eta_{\mathrm{G} 2}\right)$. Combining the above three-bin wind speed distribution with these efficiencies for a conventional wind turbine (without storage as in Fig. 3.1) yields a capacity factor of $35 \%$. This is consistent with the average power of 1.75 MW and the basic characteristics given in Tab. 3.3.

For a wind turbine with energy storage in the form of an open accumulator and spray-cooled compressed air storage, there will also be losses. The conversion from mechanical shaft energy of delivered hydraulic energy may be estimated at $90 \%$ at full load condition $\left(\eta_{\mathrm{HP} 1}\right)$ and $80 \%$ at partial load conditions $\left(\eta_{\mathrm{HP} 2}\right)$, based on Wilson's model [26] for hydraulic pump and motor. However, there will be additional losses due to compressive efficiency (based on Tab. 3.2 results) to store this energy and due to regeneration efficiency to convert the compressed air energy into electrical energy. Based on all these losses, the system annual average energy is estimated to be $1.58 \mathrm{MW}$, consistent with a $30.1 \%$ capacity factor. This is lower compared to the conventional system because of the additional losses for storage and regeneration. Therefore, the net power output is reduced (for a given size wind turbine) when spray-cooled CAES is employed.

Such a net power reduction may be worthwhile, if the overall system costs can be reduced by an even greater amount. This is possible because, the storage aspects 
importantly can allow the electrical generator size to be reduced from $5 \mathrm{MW}$ to $1.8 \mathrm{MW}$, which increases the levelization factor to 0.88. These results are shown in Tab. 3.3 along with the key aspects of both conventional and energy storage systems, where it can be seen that the generator design and placement is also simplified which can lead to further costs. The benefit of a smaller electrical generator can be the key attribute for this spraycooled CAES concept as the generator is generally the most expensive component of an on-shore wind turbine. However, the storage benefits can be enhanced for an off-shore sitting through reduced transmission line capacity (allowed by the increased levelization factor). This transmission line reduction can be critical for wind turbines that are several miles off-shore since grid connection is often the most expensive component for grouping and utilizing an off-shore wind turbine farm.

Tab. 3.3 Comparison of traditional $5 \mathrm{MW}$ offshore wind turbine to a system that adds spray-based compressed air energy storage

\begin{tabular}{|c|c|c|}
\hline & Traditional & Spray-based CAES \\
\hline Rotor diameter & $126 \mathrm{~m}$ & $126 \mathrm{~m}$ \\
\hline Hydraulic pump & Not needed & $5 \mathrm{MW}$ \\
\hline Liquid piston & Not needed & $2.2 \mathrm{MW}$ \\
\hline Open accumulator & Not needed & $35 \mathrm{MPa}, 500 \mathrm{~m}^{3}$ \\
\hline Capacity factor & 0.35 & 0.31 \\
\hline Average output & $1.75 \mathrm{MW}$ & $1.55 \mathrm{MW}$ \\
\hline Generator/Transmission capacity & $5 \mathrm{MW}$ & $1.8 \mathrm{MW}$ \\
\hline Levelization factor & 0.35 & 0.88 \\
\hline Generator rpm & Variable rpm & Constant rpm \\
\hline Gear box design & Complicated & Simple and efficient \\
\hline Gearbox weight & $120,000 \mathrm{~kg}$ & $60,000 \mathrm{~kg}$ \\
\hline Generator/hydraulic motor weight & $60,000 \mathrm{~kg}$ & $6,000 \mathrm{~kg}$ \\
\hline Other Nacelle/Drive components & $60,000 \mathrm{~kg}$ & $60,000 \mathrm{~kg}$ \\
\hline
\end{tabular}


Total Nacelle/Drive weight ${ }^{[23-25]}$

$240,000 \mathrm{~kg}$

$126,000 \mathrm{~kg}$

*bold type indicates an advantage relative to the traditional system without storage

\subsubsection{Impact on Generator Size and Nacelle Weight}

In addition to the above key benefits, this energy storage concept has several other significant benefits by replacing the electrical generator in the nacelle of Fig. 3.1 (which must be variable-speed generator owing to changing wind conditions) to an electrical generator at a sea-level platform. In particular, the platform generator can be fixed-speed that is more efficient and inexpensive (from both capital costs and maintenance costs) than a variable-speed generator. In addition, this allows elimination of the gearbox or other mechanisms used to transfer the low angular speed of the rotor (typically about 1 $\mathrm{Hz}$ for rated conditions) to a higher shaft rpm needed for efficient conversion to electricity. This can eliminate the capital costs and, more importantly, the high maintenance costs associated with gearboxes.

Another advantage to moving the generator is that it reduces nacelle mass since the generator is a large component of this mass and since hydraulic motors has relatively much lower mass for the same amount of power generation. The reduction in nacelle mass by this concept was estimated using current hydraulic motor characteristics and is shown in Tab. 3.3. A reduced nacelle-mass simplifies the structural design of the tower, which can further reduce tower mass and, thus, tower cost can be reduced.

In addition, one must consider the scenario where the energy storage system fails. In this case, the smaller generator of Tab. 3.2 (1.8 MW instead of $5 \mathrm{MW})$ would render the system inefficient and thus would generally require the whole system being taken off 
line until the storage system is working properly. However, it should be known CAES has been shown to have high reliability ${ }^{[2]}$ as has hydraulic pump and rotor ${ }^{[26]}$ technology.

In summary, open accumulator energy storage represents a viable concept for large off-shore wind turbines based on the balance of positive impact as shown by the bolded values in Tab. 3.3. However, while there are current commercial efforts to develop hydraulic driven rotors in the nacelle and CAES technology, implementation of both technologies represents a substantial change in wind turbine design and manufacturing. As such, more detailed system analysis (including capital and maintenance costs) as well as hardware validation is needed to demonstrate and quantify the potential of such energy storage to determine the overall viability of this concept. 


\section{Chapter 3 References}

[1] Sullivan P, Short W, Blair N, "Modeling the Benefits of Storage Technologies to Wind Power", American Wind Energy Association (AWEA) Wind Power 2008 Conference, Houston, Texas, June 1-4, 2008, NREL/CP-670-43510, 2008

[2] Barnes FS, Levine JG, "Large Energy Storage System Handbook", CRC Press, Boca Raton, FL, 2011

[3] Pickard WF, Hansing NJ, Shen AQ, "Can large-scale advanced-adiabatic compressed air energy storage be justified economically in an age of sustainable energy?", Journal of Renewable Sustainable Energy, Vol. 1, 2009

[4] Garrison JB, Webber ME, "An integrated energy storage scheme for a dispatchable solar and wind powered energy system", Journal of Renewable Sustainable Energy, Vol. 3, 2011.

[5] Li PY, Van de Ven JD, Sancken C, “Open Accumulator Concept for Compact Fluid Power Energy Storage”, ASME 2007 International Mechanical Engineering Congress and R\&D Exposition, Seattle, USA, IMECE2007-42580, 2007

[6] Li PY, Loth E, Simon TW, Van de Ven JD, Crane SE, "Compressed Air Energy Storage for Offshore Wind Turbines", 2011 International Fluid Power Exhibition (IFPE), Las Vegas, NV, March, 2011

[7] Van de Ven J, Li PY, "Liquid Piston Gas Compression”, Applied Energy, Vol. 86, 2009, pp. 2183-2191

[8] Qin C, Loth E, "Liquid Piston Compression with Droplet Heat Transfer", Applied Energy, Vol. 114, 2014, pp. 539-550

[9] Rice AT, "Heat Transfer Enhancement in a Cylindrical Compression Chamber By Way of Porous Inserts and the Optimization of Compression and Expansion Trajectories for Varying Heat Transfer Capabilities", M.S. Thesis, Department of Mechanical Engineering, University of Minnesota., December, 2012

[10] Saadat M, Li PY, Simon TW, "Optimal Trajectories for a Liquid Piston Compressor/Expander in a Compressed Air Energy Storage System with Consideration of Heat Transfer and Friction", 2012 American Control Conference, Montreal, Canada, June 2012

[11] United States Patent: Coney M, "Gas Compressor", Patent No. 5,771,693, June, 30, 199 
[12] Gerstmann J, Hill WS, "Isothermalization of Stirling Heat-Actuated Heat Pumps Using Liquid Pistons", $21^{\text {st }}$ Intersociety Energy Conversion Engineering Conference, Vol. 1 of 3, August, 1986

[13] Zhang C, Saadat M, Li PY, Simon TW, "Heat Transfer in a Long, Thin Tube Section of an Air Compressor: An Empirical Correlation from CFD and a Thermodynamic Modeling", ASME IMECE, Paper \#86673, Houston TX, November, 2012

[14] Zhang C, Shirazi F, Yan B, Simon TW, Li PY, Van de Ven JD, "Design of an Interrupted Plate Heat Exchanger Used in a Liquid Piston Compression Chamber for Compressed Air Energy Storage", ASME 2013 Summer Heat Transfer Conference, Minneapolis, MN, 2013

[15] Wang XF, Lefebvre AH, "Mean Drop Sizes from Pressure-Swirl Nozzles", Journal of Propulsion, Vol. 3, 1987

[16] Ide RF, "Liquid Water Content and Droplet Size Calibration of the NASA Lewis Icing Research Tunnel", NASA Technical Memorandum 102447, AIAA-90-0669, 1990

[17] Inamura T, Nagai N, "Spray Characteristics of Liquid Jet Traversing Subsonic Airstreams", Journal of Propulsion and Power, Vol. 13, 1997

[18] Ensslin C, Hoppe-Kilpper M, Rohrig K, "Wind Power Integration in Power Plant Scheduling Schemes", [online database] URL: http://www.iset.uni-kassel.de/abt/FBI/publication/00-09-25_wind_power_plant.pdf [cited May 2011]

[19] Holttinen H, "Hourly Wind Power Variations and Their Impacts on the Nordic Power System Operation", Thesis, Department of Engineering Physics and Mathematics, Helsinki University of Technology, Finland, December, 2003

[20] Stoutenburg ED, Jenkins N, Jacobson MZ, "Power Output Variations of Co-located Offshore Wind Turbines and Wave Energy Converters in California", Renewable Energy, Vol. 35, 2010, pp. 2781-2791

[21] Holttinen H et al, "Design and Operation of Power Systems with Large Amounts of Wind Power”, The IEA WIND Task 25, VTT Working Papers 82, 2007

[22] Saadat M, Li PY, "Modeling and Control of a Novel Compressed Air Energy Storage System for Offshore Wind Turbine", 2012 American Control Conference, Montreal, Canada, June 2012

[23] Jonkman J, Butterfield S, Musial W, Scott G, "Definition of a 5-MW Reference Wind Turbine for Offshore System Development", National Renewable Energy Laboratory (NREL) Technical Report, February, 2009, NREL/TP-500-38060, 2009 
[24] Loth E, Steele A, Ichter B, Selig M, Moriarty P, "Segmented Ultralight Pre-Aligned Rotor for Extreme-Scale Wind Turbines", 50th AIAA Aerospace Sciences Meeting, Nashville, TN, 2012

[25] Wikipedia, Power-to-weight ratio, [online database] URL: http://en.wikipedia.org/ wiki/Power-to-weight_ratio [cited August 2013]

[26] Wilson WE, "Rotary-Pump Theory", ASME Transactions, Vol. 68, 1946, pp. 371384 


\section{Chapter 4 Numerical Description of a Pressure-Swirl Nozzle Spray}

\subsection{Introduction}

Pressure-swirl atomizers (simplex nozzles) have many applications in both industrial and experimental processes, such as combustion, spray cooling, and tracer seeding. The downstream mass distribution of the droplets is generally critical for such applications and this distribution is dependent on the droplet sizes and the interaction of the spray with the surrounding turbulent gas.

In order to investigate the detailed structure of a spray generated by a pressureswirl atomizer, velocities of continuous and disperse phases, and sizes of spray droplets can be acquired theoretically, experimentally, or numerically. Theoretical and experimental studies have been completed by Lefebvre's research group ${ }^{[2-5]}$ in terms of spray angle, mean drop size, and radial liquid distribution, all of which are a function of fluid properties and nozzle dimensions. Similarly, Chin's group ${ }^{[6,7]}$ experimentally and analytically investigated general spray characteristics downstream of a pressure-swirl atomizer for a larger range of conditions. These studies allowed general structure of the flow but more details have been obtained recently, using Phase Doppler Particle Analyzer (PDPA) by Santolaya et al. ${ }^{[8]}$ for a pressure swirl spray nozzle. These studies provided detailed spatial information about gas flow velocities, droplet velocities for different size classes, and spatial distribution of liquid mass flow rates.

However, numerical predictions of these sprays are less common. This is because, of the complicated phenomena near the orifice which involves dense multiphase flow with high aerodynamic coupling. In particular, physics such as liquid sheet break-up, 
droplet formation, two-way coupling between turbulent flow and disperse phase, and contact interaction between droplets require intensive computationally resources to fully resolve all the spatial details. Difficulties especially come from capturing fluid instabilities and the interphase physics that cause liquid sheet to break-up into different sizes of droplets, while being subjected to anisotropic non-homogeneous turbulence. Many numerical studies have focused on tracking of the liquid/gas interface inside the atomizer in order to provide a prediction of air core and liquid sheet ${ }^{[9-11]}$. Similarly, Yeh [12] conducted turbulent flow simulations of liquid jet emanating compared different turbulent models and provided turbulent intensity at the atomizer exit for the pressureswirl atomizer. Belhadef et al. ${ }^{[13]}$ studied pressure-swirl atomization using an Eulerian method that treated the turbulent mixing as a single phase of liquid-gas mixture. Due to limits of this method, only mean droplet size and liquid axial mean velocity were predicted. In general, none of the above methods allowed a fully validated description of the two-phase flow ranging from the orifice to hundreds of orifice diameters downstream.

In this study, a two-phase computational fluid dynamic (CFD) simulation is implemented just downstream of the liquid sheet break-up region of a pressure-swirl nozzle to allow the simulation to focus on the more computationally practical spray structure evolution. Herein, previous PDPA experimental data after the break-up region are employed as input boundary condition data. Turbulent characteristics of continuous phase are modeled at the same boundary based on empirical correlations of jet flow. Thus, spray structure and droplet spatial distribution resulting from coupling of disperse and continuous phases can be simulated in the far downstream domain. This novel approach to simulating a pressure-swirl nozzle spray allows moderate mesh resolution 
and CPU time while avoiding the complicated break-up region details. Importantly, by adapting inflow boundary conditions, this methodology may be applied to other pressureswirl nozzle spray cases to predict downstream spray structure.

\subsection{Flow Physics and Numerical Methodology}

In the present study, the Eulerian numerical approach is chosen for the gas phase and the Lagrangian approach for the liquid phase. To ensure both numerical stability and low computational cost, the continuous phase (ambient gas) is solved using the Reynoldsaveraged Navier-Stokes (RANS) equations with a standard k- $\varepsilon$ model, while the dispersed phase (droplet) is solved by trajectory discretization with a discontinuous random walk (DRW) model to incorporate turbulent fluctuations.

In the standard $\mathrm{k}-\varepsilon$ model ${ }^{[14]}$, the turbulent kinetic energy $k$ and its rate of dissipation $\varepsilon$ are solved in two transport equations:

$$
\begin{aligned}
& \frac{\partial}{\partial t}\left(\rho_{g} k\right)+\frac{\partial}{\partial x_{i}}\left(\rho_{g} k u_{i}\right)=\frac{\partial}{\partial x_{j}}\left[\left(\mu_{g}+\frac{\mu_{t}}{\sigma_{k}}\right) \frac{\partial k}{\partial x_{j}}\right]+G_{k}+G_{b}-\rho_{g} \varepsilon-Y_{M} \\
& \frac{\partial}{\partial t}\left(\rho_{g} \varepsilon\right)+\frac{\partial}{\partial x_{i}}\left(\rho_{g} \varepsilon u_{i}\right)=\frac{\partial}{\partial x_{j}}\left[\left(\mu_{g}+\frac{\mu_{t}}{\sigma_{k}}\right) \frac{\partial \varepsilon}{\partial x_{j}}\right]+C_{1 \varepsilon} \frac{\varepsilon}{k}\left(G_{k}+C_{3 \varepsilon} G_{b}\right)-C_{2 \varepsilon} \rho_{g} \frac{\varepsilon^{2}}{k}
\end{aligned}
$$

The turbulent viscosity $\mu_{t}$ appearing on the right-hand side expressions is calculated by:

$$
\mu_{t}=C_{\mu} \rho_{g} \frac{k^{2}}{\varepsilon}
$$


In equation 4-1 and 4-2, $\rho$ is density, $u$ refers to gas velocity, and the subscript $g$ indicates a gas-phase property while the turbulent kinetic energy generation and distraction terms $\left(G_{k}, G_{b}\right.$, and $\left.Y_{M}\right)$ are described in Ref. 14. Herein, conventional values are used for the constants and non-dimensional numbers: $C_{1 \varepsilon}=1.44, C_{2 \varepsilon}=1.92, C_{\mu}=$ $0.09, \sigma_{k}=1.0, \sigma_{\varepsilon}=1.3$. Note that the spray flow field is assumed to not impact the gasphase turbulence, i.e. there are no inter-phase coupling terms for Eq. 4-1 and Eq. 4-2 consistent with recent work by Huang et al. ${ }^{[15]}$. However, the impacts between mean continuous phase and discrete phase velocities are coupled by momentum exchange, where the latter is described below.

To describe trajectory of a discrete phase particle dominated by drag and gravity forces, the equation of motion for droplets with centroid velocity $v_{i}$ is expressed in a Lagrangian reference frame ${ }^{[16]}$ as:

$$
\frac{d v_{i}}{d t}=F_{D}\left(u_{i}-v_{i}\right)+\frac{\left(\rho_{d}-\rho_{g}\right)}{\rho_{d}} g_{i}
$$

In this expression, the subscript $d$ indicates a droplet property and $F_{D}$ is the drag force per particle mass per relative velocity defined as

$$
F_{D}=\frac{3 \mu_{g} C_{D} \operatorname{Re}}{4 \rho_{d} d^{2}}
$$

The value in the numerator Re is the relative Reynolds number, which is defined in terms of the magnitude of relative velocity $w$ as

$$
\operatorname{Re} \equiv \frac{\rho_{g} d w}{\mu_{g}}
$$




$$
\begin{aligned}
& w=\sqrt{w_{i} w_{i}} \\
& w_{i}=v_{i}-u_{i}
\end{aligned}
$$

The last expression for $w_{i}$ is the component of relative velocity in the $i$ direction. A spherical droplet assumption is reasonable herein since the Weber numbers are less than unity, since a unity Weber number corresponds to droplet diameters of 400 microns [16]. Thus, the drag coefficient $C_{D}$ for spheres is used and taken from Morsi and Alexander ${ }^{[17]}$.

The instantaneous gas velocity in Eqs. 4-4 and 4-6 can be obtained in terms of mean and fluctuation values:

$$
u_{i}=\bar{u}_{i}+u_{i}^{\prime}
$$

The mean value (first term on right-hand side) is obtained from RANS solution. For the fluctuation velocity (second term on right-hand side), synthetic turbulence must be used to properly capture turbulent diffusion. Herein, the discrete random walk (DRW) model of Ref. 18 is employed, which requires a random number $(\sigma)$, an eddy lifetime $\left(\tau_{\Lambda}\right)$, and a velocity perturbation strength $\left(u_{r m s}\right)$. For small droplets with small relative velocities, the characteristic lifetime of eddies can be based on the integral time in DRW model. Thus, in DRW model, the gas velocity fluctuations can be computed in each integral time as

$$
u_{i}^{\prime}=u_{r m s} \sigma_{i}
$$




$$
\begin{aligned}
& u_{r m s}=\left(\frac{2}{3} k\right)^{1 / 2} \\
& \tau_{\Lambda}=C_{\tau} \frac{k}{\varepsilon}
\end{aligned}
$$

In Eq. 4-8a, $\sigma$ represents three independent Gaussian random numbers and $C_{\tau}$ of Eq. $4-8 \mathrm{c}$ is the eddy timescale coefficient, whose calibrated value is $0.21^{[18]}$. A new random number is generated when the droplet path duration exceeds $\tau_{\Lambda}$ (eddy life time) or $\tau_{\text {tra }}$ (eddy traversal time), where the latter is obtained as

$$
\tau_{t r a, i}=\frac{\Lambda_{i}}{w}
$$

where $\Lambda$ is turbulence integral length scale, which is based on the local turbulent kinetic energy and dissipation values ${ }^{[18]}$.

To validate this approach in terms of predicting turbulent flow decay and particle lateral diffusion, Snyder and Lumley's classical experiment ${ }^{[19]}$ was utilized. In this experiment, nearly homogenous turbulent flow was generated by grids and the spatial diffusion of four different kinds of particles were measured. The RANS-based CFD employed an inflow boundary condition based on measurements of $\bar{u}, k$ and $\varepsilon$ at $60 \mathrm{~mm}$ downstream of the grid. The CFD predictions of the evolution and decay of these turbulent flow properties are compared to the experimental data in Fig. 4.1, and the agreement can be regarded as excellent. The experimental and predicted lateral dispersions of four different particles released in this flow are shown in Fig. 4.2a. As noted in the caption, the particle inertias can be characterized in terms of a response time scale $\left(\tau_{p}\right)$ defined as 


$$
\tau_{d}=\frac{\rho_{d} d^{2}}{18 \mu_{g}}
$$
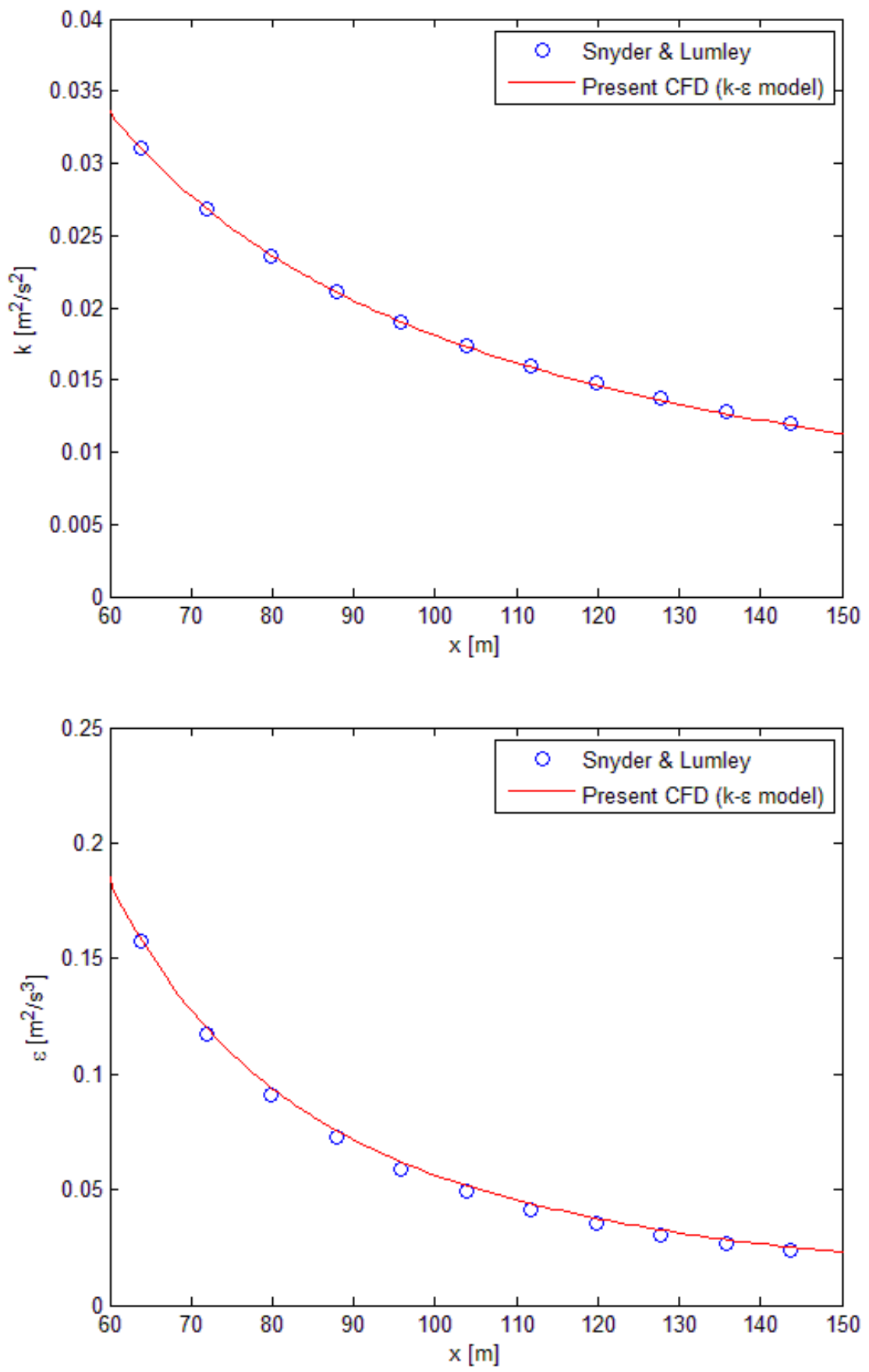

Fig. 4.1 Comparison of numerical results to the experiment of Snyder and Lumley ${ }^{[19]}$ for a) turbulent kinetic energy and b) dissipation rate 

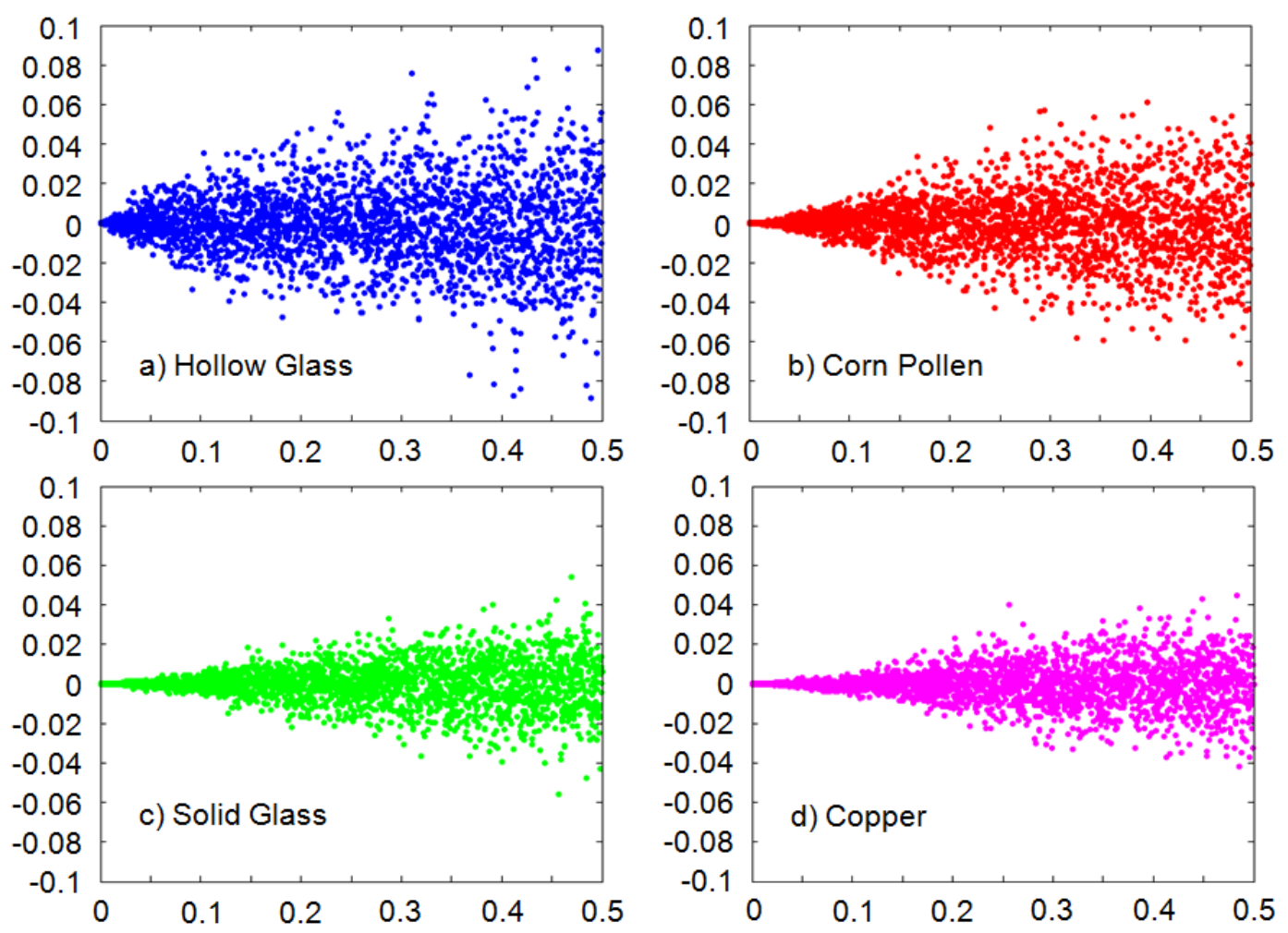

Fig. 4.2 Numerical results of dispersion of four kinds of particles (with increasing particle response time): a) hollow glass $(0.00174 \mathrm{sec})$, b) corn pollen $(0.0223 \mathrm{sec})$, c) solid glass $(0.0557 \mathrm{sec})$ and d) copper $(0.0594 \mathrm{sec})$ 


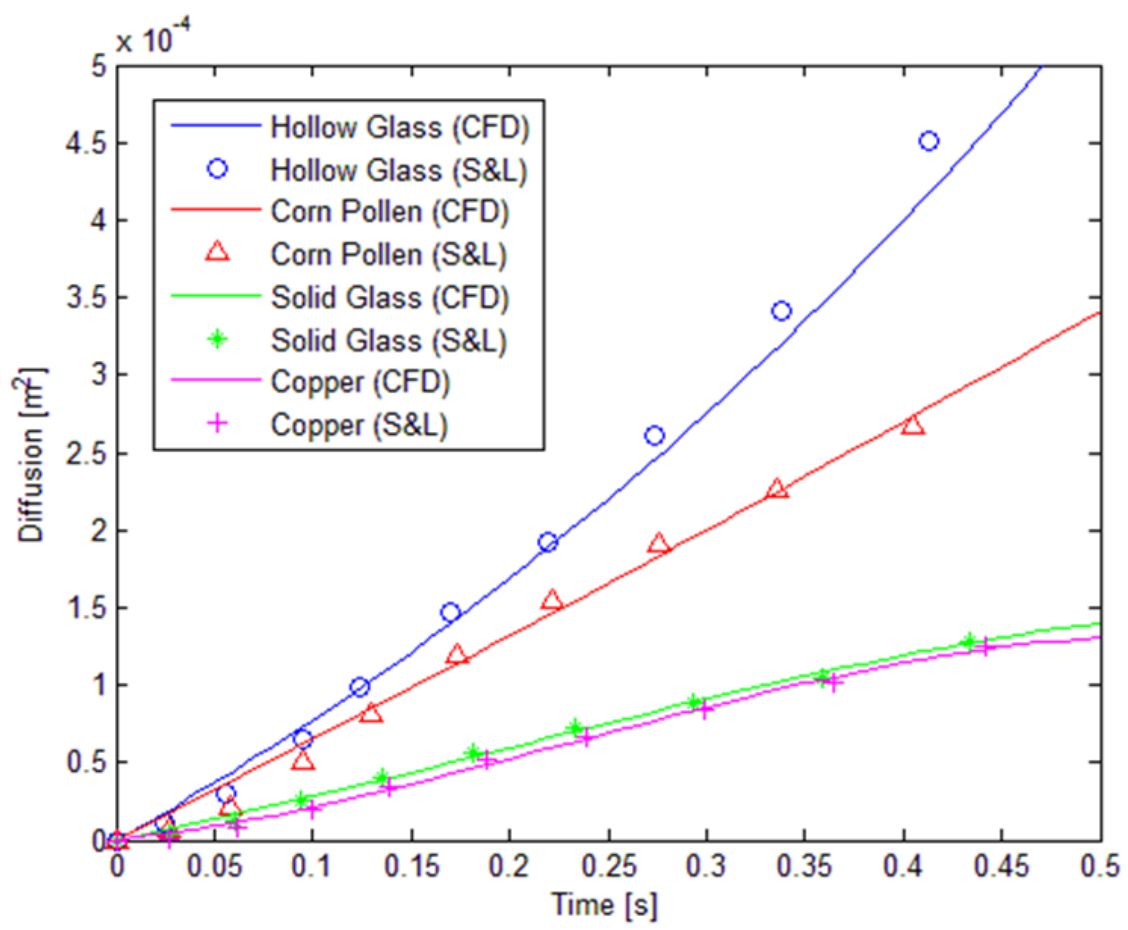

Fig. 4.3 Diffusion comparison of numerical results to the experiment of Snyder and Lumley ${ }^{[19]}$

It can be seen in Figs. 4.2 and 4.3 that radial diffusion increases as response time (and inertia) decreases. The present DRW-based CFD results indicated good agreement for short-time and long-time turbulent particle diffusion. Based on the above, this validated numerical approach is utilized below to predict pressure-swirl liquid spray structure and droplet dispersion. 


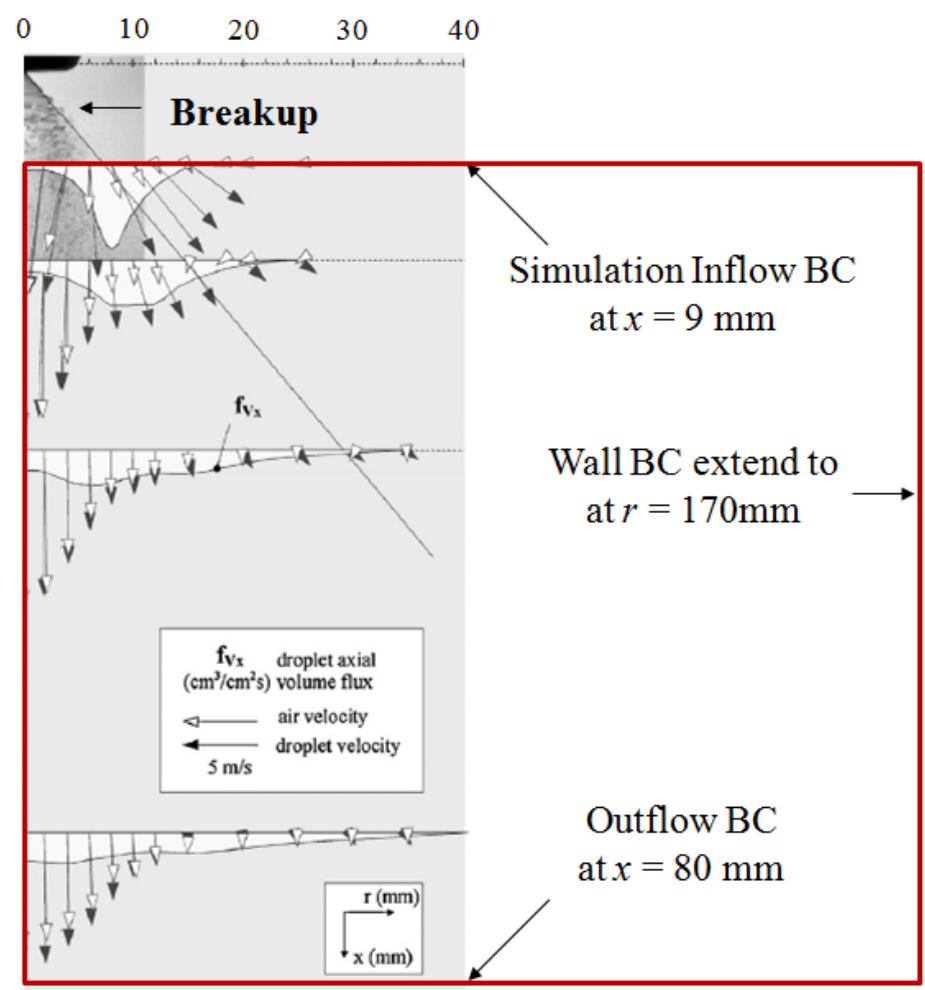

Axisymmetric Simulation Zone

Fig. 4.4 Sketch of simulation domain based on figure from Santolaya et al. ${ }^{[8]}$

\subsection{Axisymmetric Simulation}

To simulate structure of liquid spray after the break-up region, an axisymmetric zone is defined based on the experimental flow field ${ }^{[8]}$ as shown in Fig. 4.4. A cylindrical coordinate system is chosen with the nozzle orifice as the origin with $r$ for radial and $x$ for axial distance. Inflow and outflow boundary conditions are set at $x=9$ $\mathrm{mm}$ and $x=80 \mathrm{~mm}$ respectively, while a wall boundary is set at $r=170 \mathrm{~mm}$. Note that in the experiment, the spray was operating in a square cross-section tank of $340 \mathrm{~mm}$ by 340 $\mathrm{mm}$ cross-section rather than cylinder, but this geometry difference may be reasonable neglected because the spray structure is generally contained to be within $r=40 \mathrm{~mm}$ as shown in Fig. 4.4. In the simulation, radial spatial distributions of both the continuous 
and disperse phase flow properties are introduced at inflow boundary $(x=9 \mathrm{~mm})$, including velocity and turbulence of the gas as well as mass flux, velocity, and turbulence of the drops. Details of these distributions are described below.

For the continuous phase, the experimental gas axial velocity at $x=9 \mathrm{~mm}$ was fitted with a hyperbolic sech function as shown in Fig. 4.5a, and the gas radial velocity was similarly fitted with an exponential bounded quintic polynomial. These velocity profiles satisfied a $0.15-\mathrm{m} / \mathrm{s}$ gas co-flow velocity, consistent with the experimental test conditions. The profiles of gas turbulent kinetic energy and dissipation rate are calculated based on turbulent jet curve fits of experimental data ${ }^{[21]}$ with a form:

$$
\begin{aligned}
& p(\eta)=\left(C_{0}+C_{2} \eta^{2}+C_{4} \eta^{4}+C_{6} \eta^{6}\right) e^{-A \eta^{2}} \\
& \eta=\frac{r-r_{0}}{x-x_{0}}
\end{aligned}
$$

In this expression, $\eta$ is a normalized position, and $r_{0}$ and $x_{0}$ are orifice radius and location respectively. It was found that the gas mean axial velocity of the pressure swirl nozzle at $x=9 \mathrm{~mm}$ was nearly identical to that for the turbulent jet flow of Ref. 21 at $x=$ $32 \mathrm{~mm}$. Thus, the profiles of turbulent kinetic energy and dissipation rate at $32 \mathrm{~mm}$ downstream of the jet in Ref. 21 were employed as inflow boundary condition at $x=9$ mm downstream of the pressure swirl nozzle. This was accomplished by using the $C_{i}$ and $A$ listed in Ref. 21.

For the disperse phase, three-size (small, medium, and large) spherical droplets are chosen to represent the polydisperse distribution. For each size class, a single diameter is used in the simulations: small at $7.5 \mu \mathrm{m}$, medium at $25 \mu \mathrm{m}$, and large at 55 
$\mu \mathrm{m}$. This preserves a 30.6- $\mu \mathrm{m}$ Sauter mean diameter (SMD), consistent with the measured SMD at $x=9 \mathrm{~mm}$. The droplet mean axial velocity profiles for the CFD for all three classes are obtained by fitting the experimental data at $x=9 \mathrm{~mm}$ as shown in Fig. 4.5a. However, it was noted that the flow simulations yielded higher fidelity if a random velocity perturbation was also included, especially for large droplets, to reflect initial turbulence. Because the droplet velocity fluctuations were not measured, it was assumed that droplet velocity fluctuation are equal to that of gas velocity at $x=9 \mathrm{~mm}$, yielding the instantaneous velocities as shown in Fig. 4.5b.

To specify the radial variation of the droplet mass flow rate at this computational inflow plane, an assumption was needed regarding the distribution among the three sizes. In the measurements, the mass flux based on the sum of the three classes at $x=9 \mathrm{~mm}$ was $0.509 \mathrm{~cm}^{3} / \mathrm{s}$, which is a $34.4 \%$ deficit of unaccounted flux relative to the total injected liquid volumetric flow of $0.77 \mathrm{~cm}^{3} / \mathrm{s}$. Therefore, it is herein assumed that the relative flow rate percentages of three classes are the same as measured (small class at $2 \%$, medium class at 57\%, and large class at $41 \%$ ), and the mass fluxes for each case were then scaled so that the total flow rate was $0.77 \mathrm{~cm}^{3} / \mathrm{s}$, consistent with the experimental total volumetric flow. Based on particle velocity profile, liquid flow rate, the radial distribution of a particle mass flow rate at inflow boundary is shown as Fig. 4.6. Thus, all particles are injected at $9 \mathrm{~mm}$ with a specified droplet velocity profile (Figs. 4.5) and radial mass flow rate distribution (Fig. 4.6). 

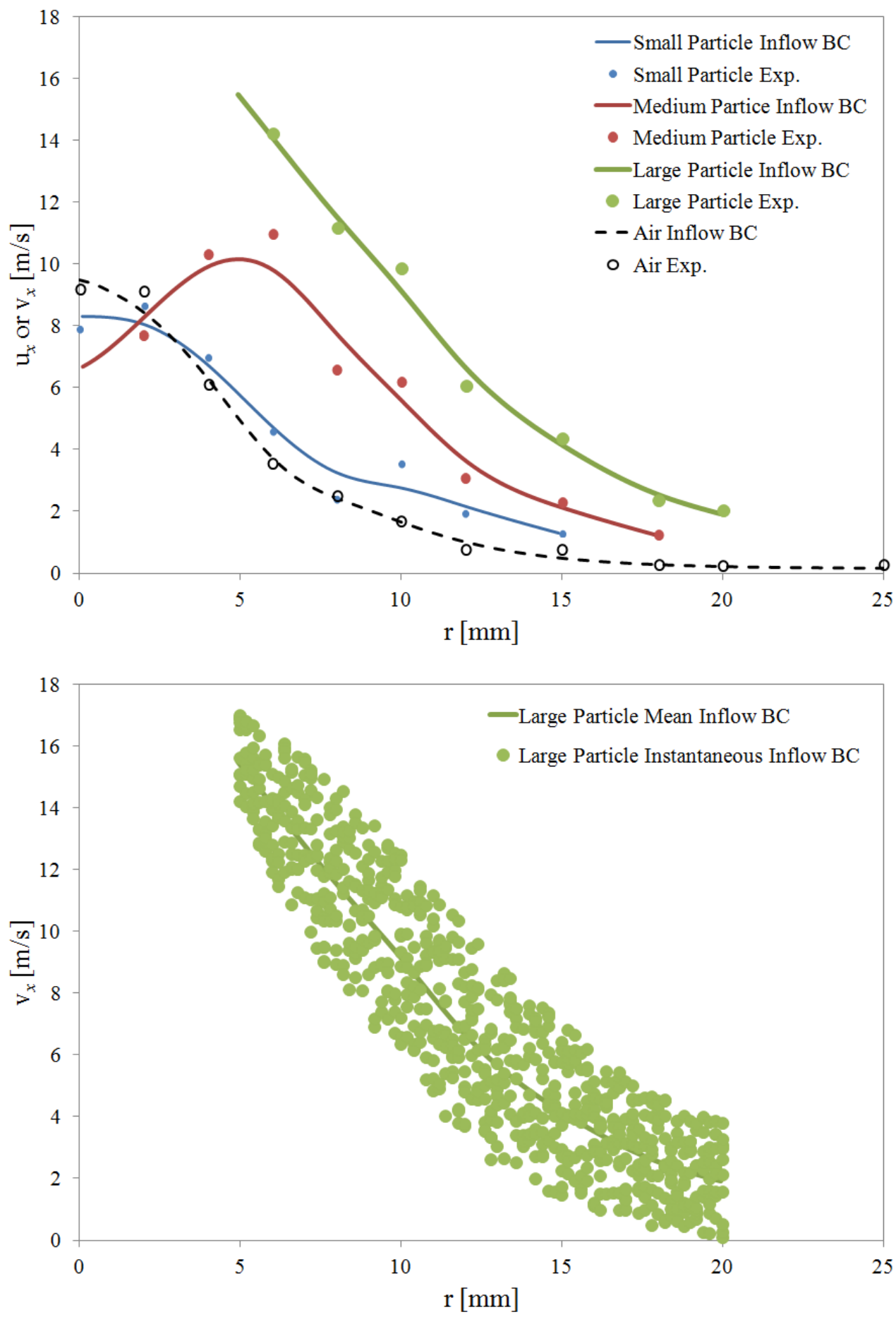

Fig. 4.5 Axial velocity input at $x=9 \mathrm{~mm}$ : a) mean velocity for both gas and particles, b) mean and fluctuating velocities for large particles 


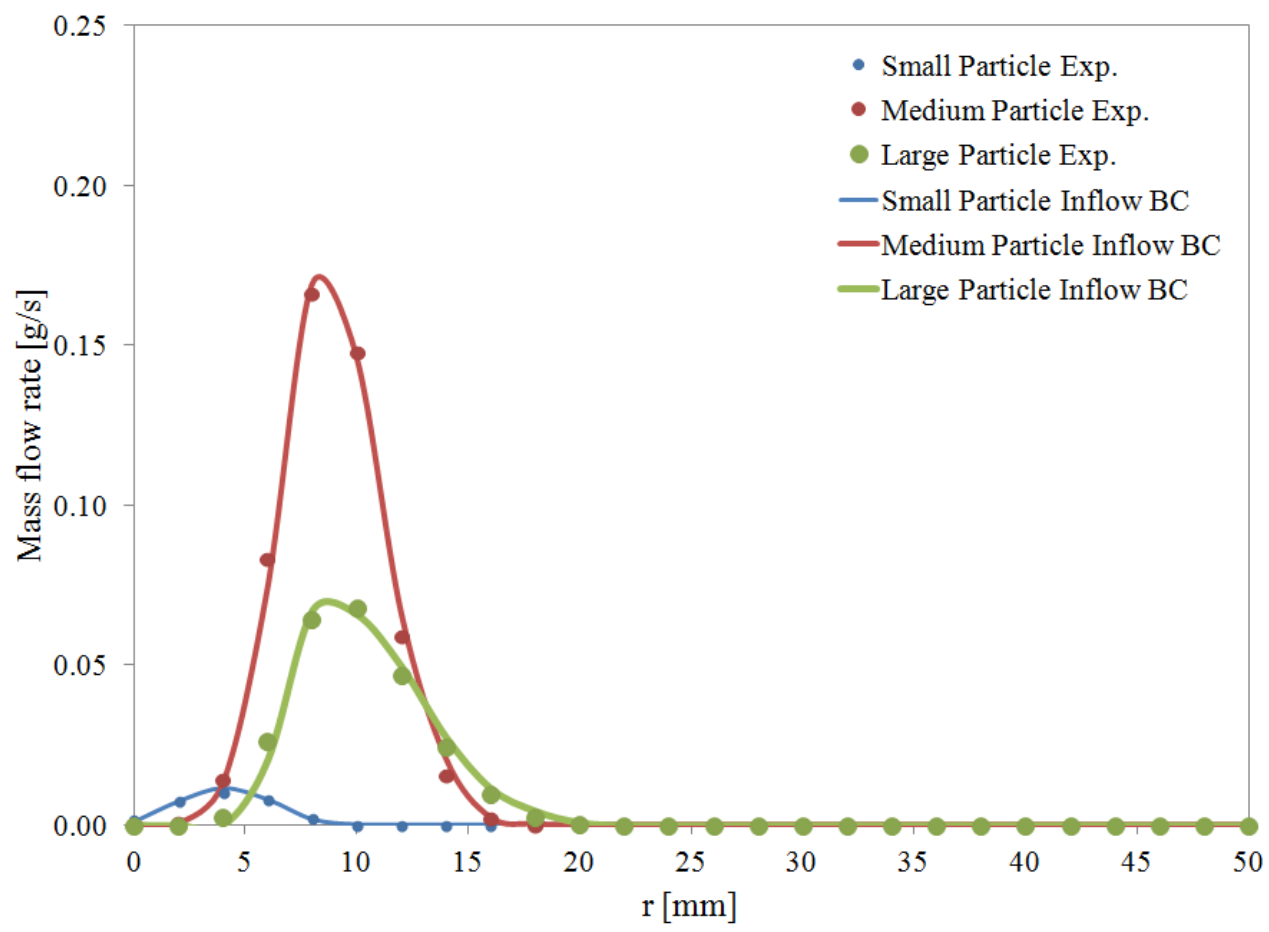

Fig. 4.6 Definition of droplet mass flux inflow boundary conditions as a function of drop size

In CFD simulation, mesh sizes of $\Delta x=\Delta r=10^{-3} \mathrm{~m}$ and time step of $10^{-3} \mathrm{~s}$ are chosen based on grid and temporal resolution studies. In each time step, 2410 parcels (groups of droplets that move at the same speed) are injected into simulation domain. These conditions are chose such that a two-fold improvement in grid, timestep and parcel resolution resulted in less than $1 \%$ variation in downstream gas or droplet mean velocity. The computational resources required for this approach are therefore rather modest while ensuring accuracy and computational efficiency. In order to predict the mean spray structure, the droplet trajectories were time-averaged (after the initial transient phase for which the first droplets exited the computational domain) using a volume integral of particle concentration. To achieve statistically stationary results, this sampling was 
averaged over 1000 time steps, i.e. one second. The gas flow solution had also converged at that time to a steady-state coupled flow.

\subsection{Results}

Predictions of the axisymmetric gas flow after convergence are shown in terms of the velocity field (Fig. 4.7a) and turbulent kinetic energy field (Fig. 4.7b). The velocity field of continuous phase indicates a high speed core in the center, where gas is accelerated by injected particles, and this velocity decays in both the downstream and outward (radial) directions as expected due turbulent diffusion of a jet. In Fig. 4.7a, it also can be seen difference between cases with and without droplet injection is small. Figure $7 \mathrm{~b}$ shows that the turbulent kinetic energy is high in the core region due to high shear rates and interphase coupling, since the particle concentration is relatively high in this region. Thus, it is reasonable to specify inflow conditions at 9-mm downstream avoiding simulation of break-up.

Figure 4.8a shows the particle axial velocities at $x=18 \mathrm{~mm}$. In general, the particle velocities are similar to the gas velocities, but the larger particle velocities tend to have the highest velocities. This is due to the higher inertia and response times of these droplets that prevents them from slowing down as quickly as that of the smaller drops and that of the gas. The medium and smaller droplets tend to more closely follow the gas velocity, especially at the far downstream locations. There is a good agreement between CFD results and experimental data sets for droplet velocity. To investigate the lateral diffusion of the drops, the mass flow rates are shown in terms or radial distribution at different downstream locations (Fig. 4.8b). In general, the small droplets tend to stay closer to the centerline while the largest droplets tend to be more spread out. Their 
increased radial can be attributed to their initial radial velocity and their high inertias coupled with turbulent diffusion.
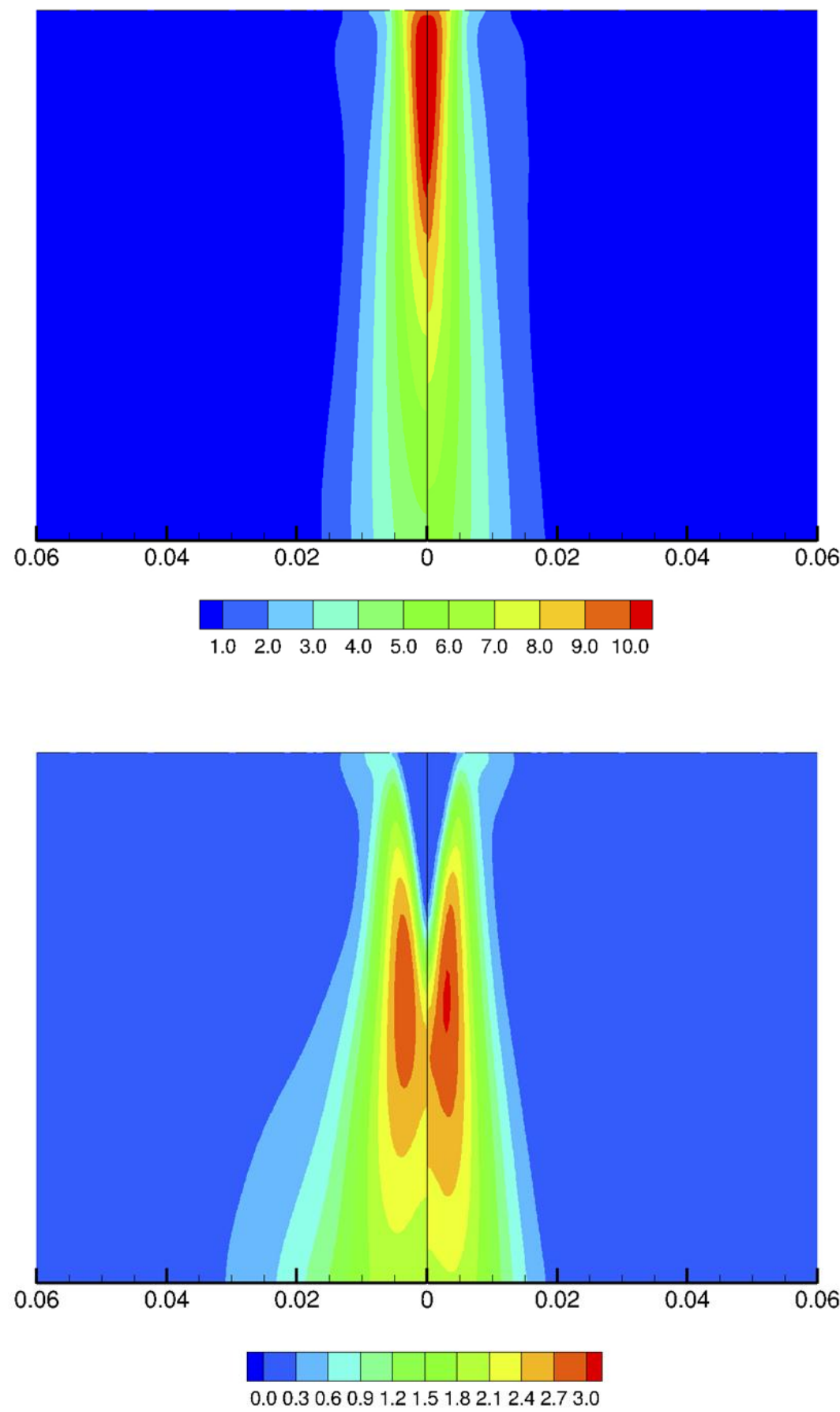

Fig. 4.7 Simulation result (left: without particle; right: with particles): a) contours of gas velocity magnitude $(\mathrm{m} / \mathrm{s})$, and b) contours of turbulent kinetic energy $\left(\mathrm{m}^{2} / \mathrm{s}^{2}\right)$ 

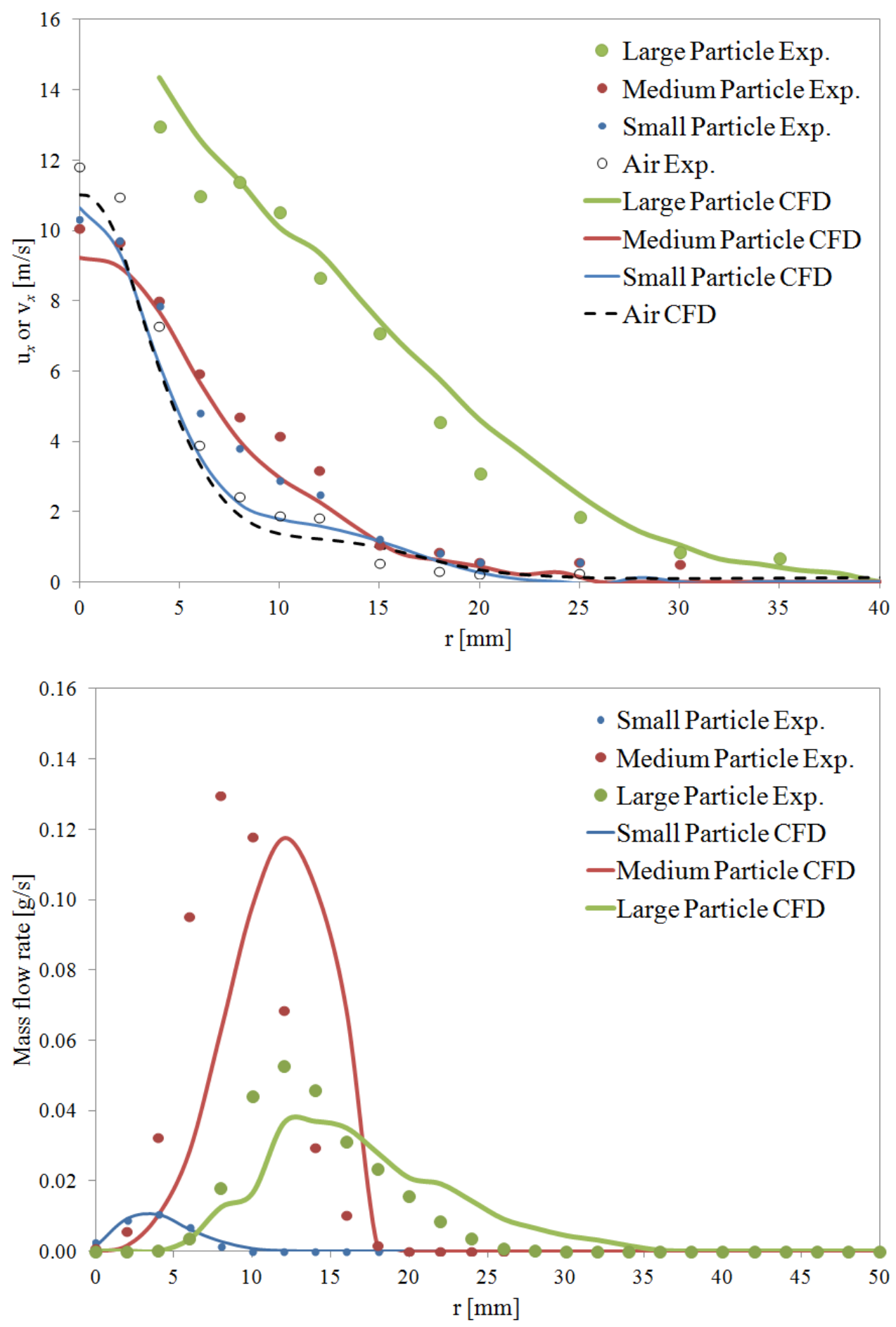

Fig. 4.8 Comparison between CFD and experiment at $\mathrm{x}=18 \mathrm{~mm}$ for: a) droplet and gas axial velocity, b) droplet mass flux rate 
In Fig. 4.9a, all axial velocities get close to each other, except for large droplets. The simulations predict the velocities similarly to the experiments. In Fig. 4.9b, it can be seen a smooth peak shaped flux distribution is formed for medium particles, indicating a well developed spray sub-structure for medium size. For large particles, the sub-structure is still developing and shows an unsmooth radial flux distribution.

When it comes to Fig. 4.10a, axial velocity predictions are smaller than experiment data. Based on conservation of momentum, radial velocities increase. Looking at Fig. 4.10b, all particles sizes show a smooth peak-shaped flux radial distribution, indicating that the spray is well-developed. In general, the small droplets tend to stay closer to the centerline while the largest droplets tend to be more spread out. Their increased radial can be attributed to their initial radial velocity and their high inertias coupled with turbulent diffusion. The predicted mass flow radial distributions are similar shape to the experiments but tend to over-predict the radial spread of the droplets. This again suggests that the simulations are over-predicting the turbulent diffusion of the gas. This is primarily attributed to the lack of collision physics in the CFD prediction. In particular, smaller droplets which collide near the centerline to make larger droplets will tend to increase the mass flux of larger droplets in this region. A secondary effect which may have caused this difference is the lack of inter-phase coupling in the gas turbulence evolutionary equations (Eqs. 4-1 and 4-2) since it is known that droplets much smaller than the integral eddy sizes (as is the case here) tend to dampen turbulence as discussed by Sundaram \& Collins ${ }^{[22]}$. Unfortunately, such coupling is not well understood ${ }^{[23]}$ so that there is no robust model for this for CFD and thus is typically ignored ${ }^{[15]}$. Such 
turbulence coupling is an outstanding challenge for these flows that should motivate further studies.
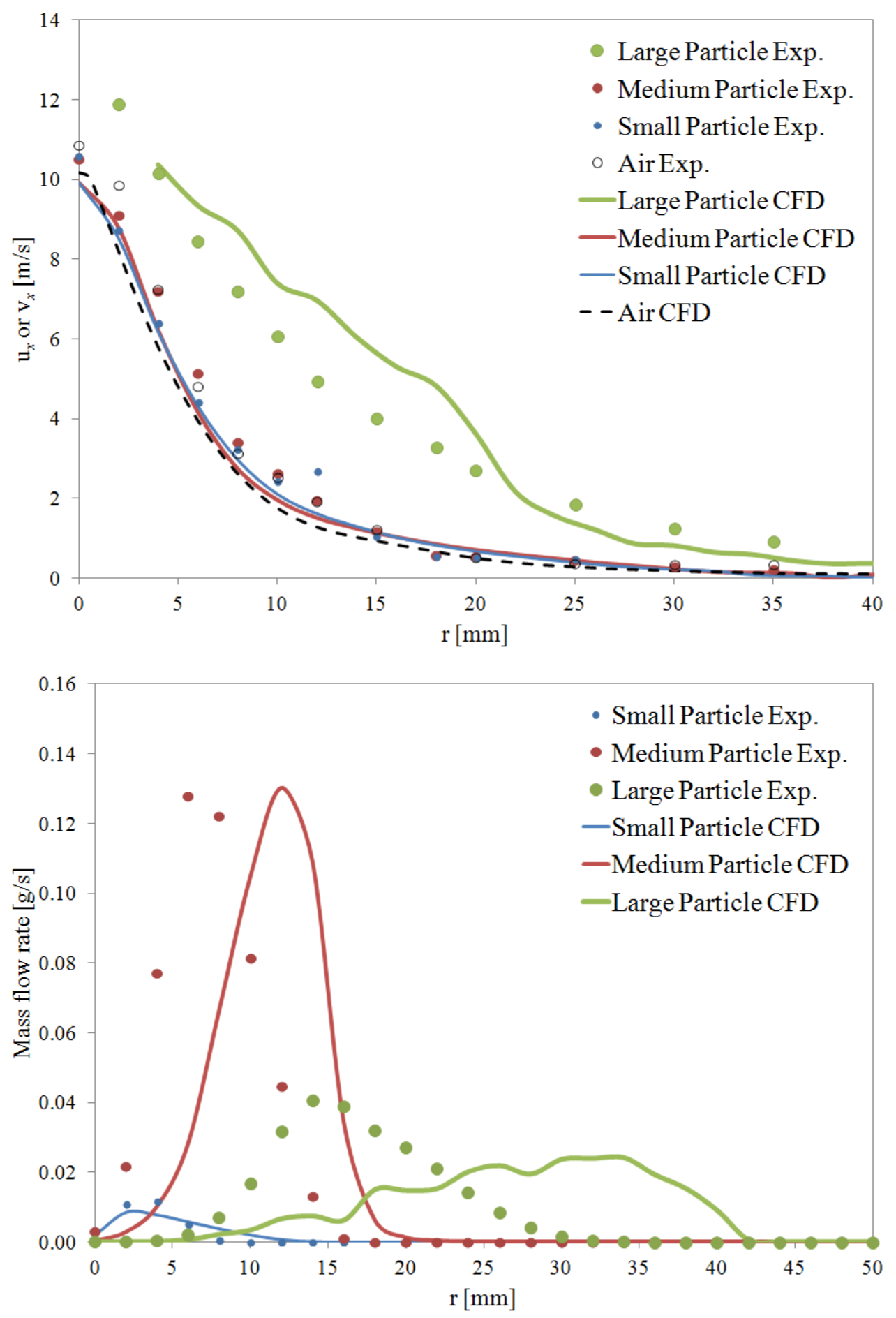

Fig. 4.9 Same caption as Fig. 4.8, except for $x=36 \mathrm{~mm}$ 

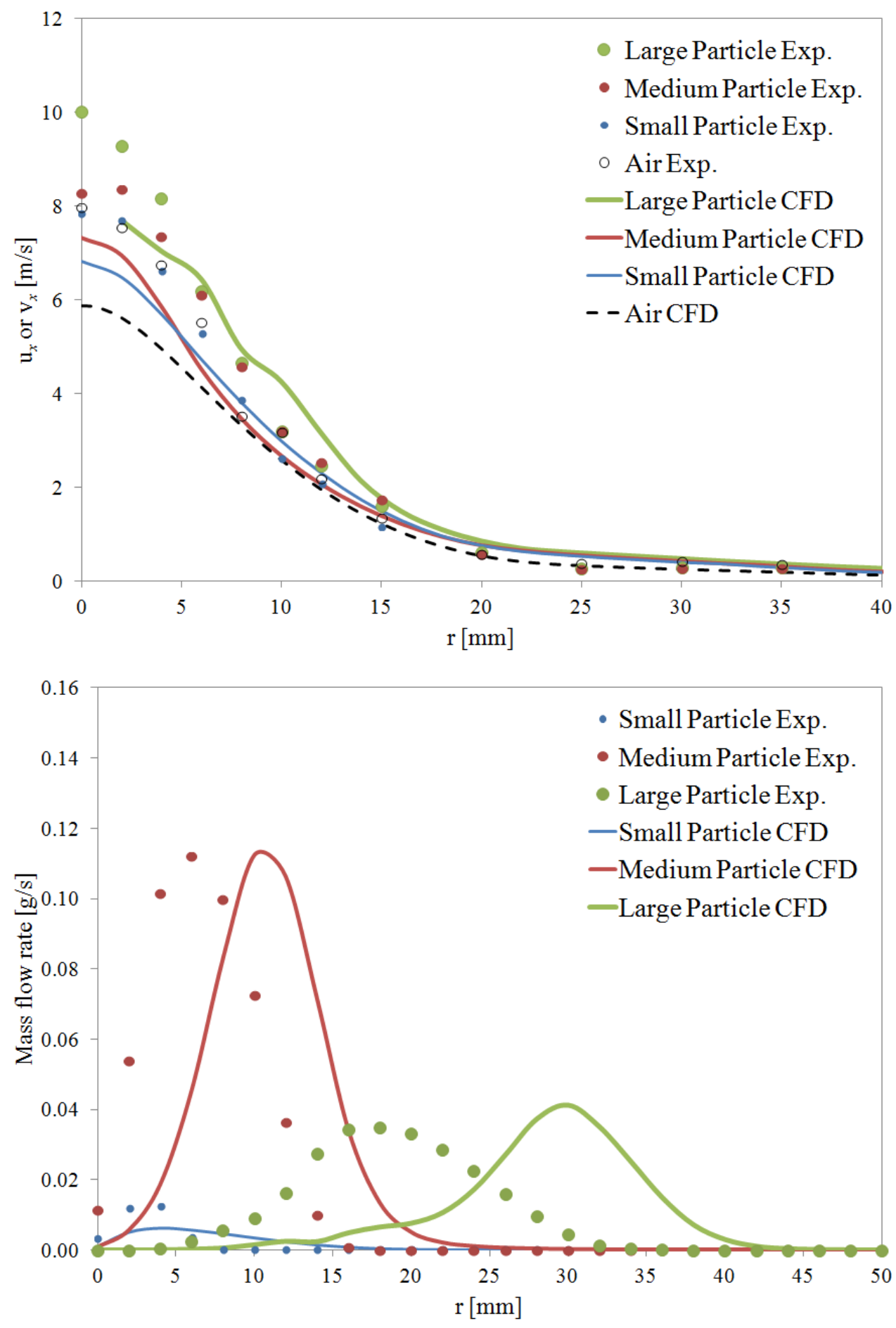

Fig. 4.10 Same caption as Fig. 4.8, except for $x=72 \mathrm{~mm}$ 
Finally, the droplet kinetic energy was investigated to gain insight into the controlling physics. Since no experimental results were available, the CFD predictions of droplet turbulence were compared to modeled results which employ a combination of particle kinetic theory and the predicted gas flow turbulence. In order to describe lateral velocity fluctuation of the droplets, the particle radial velocity fluctuation is defined as

$$
v_{r, r m s}^{\prime}=\left(v_{r}-\overline{v_{r}}\right)_{r m s}
$$

where $v_{r}$ indicates particle radial velocity, ( )' indicates a fluctuation from the mean value and ( ) rms indicates the root-mean-square value. This radial particle kinetic energy can be compared to the gas turbulent kinetic energy based on theory in Ref. 20 which employs the integral-scale particle Stokes number, which is the ratio of the particle inertia (Eq. 4-10) to turbulent integral time-scale (Eq. 4-8c):

$$
\begin{aligned}
& \frac{v_{r, r m s}^{\prime}}{u_{r, r m s}^{\prime}}=\frac{1}{\sqrt{1+\mathrm{St}_{\Lambda}}} \\
& \mathrm{St}_{\Lambda}=\frac{\tau_{d}}{\tau_{\Lambda}}
\end{aligned}
$$

The ratio of particle to gas velocity fluctuations (Eq. 4-13a) tends to unity when the Stokes number trends to zero, i.e. the particle turbulence will tend to the gas turbulence as the droplet size and inertia becomes very small so that it simply follows and mimics the gas motion. As shown in Fig. 11, the simulations of the droplet velocity fluctuations are consistent with theory and the predicted gas velocity fluctuations. This indicates that the particle turbulent dispersion model provide reasonable results. The results also show that the small droplets are close to equilibrium with the gas at this far 
downstream location while the large drops have substantially reduced fluctuations (relative to the gas) in the core region but tend to equilibrium at large radial locations.

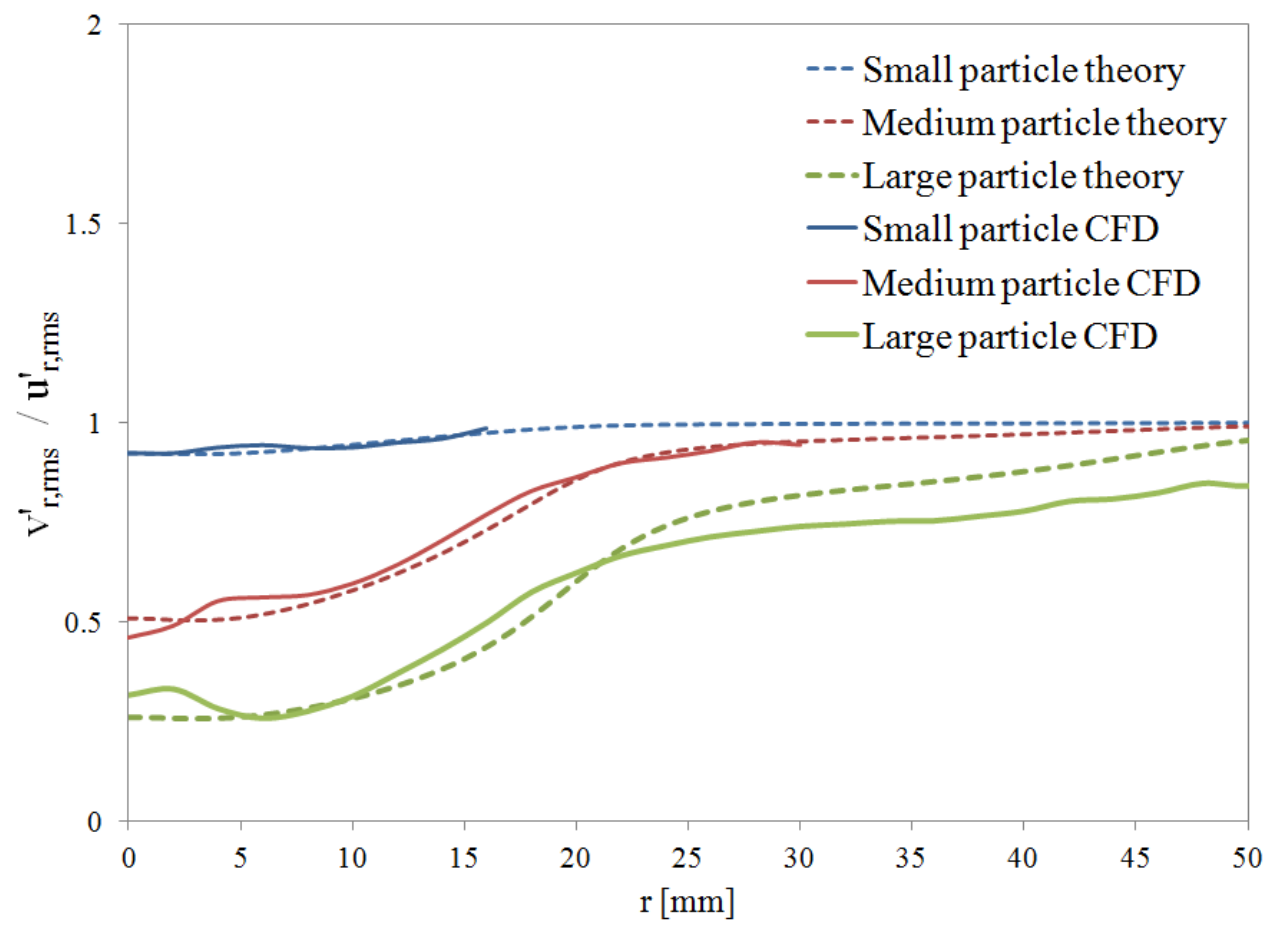

Fig. 4.11 Droplet radial velocity fluctuations at $x=72 \mathrm{~mm}$ 


\section{Chapter 4 References}

[1] Lefebvre AH. Atomization and Sprays. Hemisphere, New York, 1989.

[2] Rizk NK, Lefebvre AH. Spray Characteristics of Simplex Swirl Atomizers. Progress in astronautics and aeronautics (AIAA), Vol. 95, 1984, pp. 563-580.

[3] Wang XF, Lefebvre AH. Mean Drop Sizes from Pressure-Swirl Nozzles. Journal of Propulsion, Vol. 3, No. 1, 1987, pp. 11-18.

[4] Ortman J, Lefebvre AH. Fuel Distributions from Pressure-Swirl Atomizers. Journal of Propulsion, Vol. 1, No. 1, 1985, pp. 11-15.

[5] Chen SK, Lefebvre AH, Rollbuhler J. Factors Influencing the Effective Spray Cone Angle of Pressure Swirl Atomizers. Journal of Engineering for Gas Turbines and Power, Vol. 114, 1992, pp. 97-103.

[6] Chin JS, Nickolaus D, Lefebvre AH. Influence of Downstream Distance on the Spray Characteristics of Pressure-Swirl Atomizers. Journal of Engineering for Gas Turbines and Power, Vol. 108, 1986, pp. 219-224.

[7] Zhao YH, Li WM, Chin JS. Experimental and Analytical Investigation on the Variation of Spray Characteristics Along Radial Distance Downstream of a Pressure Swirl Atomizer. Journal of Engineering for Gas Turbines and Power, Vol. 108, 1986, pp. 473-478.

[8] Santolaya JL, Aisa LA, Calvo E, Garcia I, Garcia JA. Analysis by Droplet Size Classes of the Liquid Flow Structure in a Pressure Swirl Hollow Cone Spray. Chemical Engineering and Processing, Vol. 49, 2010, 125-131.

[9] Jeng SM, Jog MA, Benjamin MA. Computational and Experimental Study of Liquid Sheet Emanating from Simplex Fuel Nozzle. AIAA Journal, Vol. 36, No. 2, 1998, pp. 201-207.

[10] Datta A, Som SK. Numerical Prediction of Air Core Diameter, Coefficient of Discharge and Spray Cone Angle of a Swirl Spray Pressure Nozzle. International Journal of Heat and Fluid Flow, Vol. 21, 2000, pp. 412-419.

[11] Dash SK, Halder MR, Peric M, Som SK. Formation of Air Core in Nozzles with Tangential Entry. Journal of Fluids Engineering, Vol.123, 2001, pp. 829-835.

[12] Yeh C. Numerical Simulation of a Turbulent Liquid Jet Emanating from a PlainOrifice Atomizer and a Pressure-Swirl Atomizer. Numerical Heat Transfer, Part A, Vol. 51, 2007, pp. 1187-1212. 
[13] Belhadef A, Vallet A, Amielh M, Anselmet F. Pressure-Swirl Atomization: Modeling and Experimental Approaches. International Journal of Multiphase Flow, Vol. 39, 2012, pp. 13-20.

[14] Launder BE, Spalding DB. Lectures in Mathematical Models of Turbulence. Academic Press, London, England, 1972.

[15] Huang LX, Kumar K, Mujumdar AS. A Comparative Study of a Spray Dryer with Rotary Disc Atomizer and Pressure Nozzle Using Computational Fluid Dynamic Simulations. Chemical Engineering and Processing, Vol. 45, 2006, pp. 461-470.

[16] Kersey J, Loth E, Lankford D. Effects of Evaporating Droplet on Shock Waves. AIAA Journal, Vol. 48, 2010, pp. 1975-1985.

[17] Morsi SA, Alexander AJ. An Investigation of Particle Trajectories in Two-Phase Flow Systems. Journal of Fluid Mechanics, Vol. 55, No. 2, 1972, pp. 193-208.

[18] Bocksell TL, Loth E. Random Walk Models for Particle Diffusion in Free-Shear Flows. AIAA Journal, Vol. 39, No. 6, 2001, 1086-1096.

[19] Snyder WH, Lumley JL. Some Measurements of Particle Velocity Autocorrelation Functions in a Turbulent Flow. Journal of Fluid Mechanics, Vol. 48, 1971, pp. 41-71.

[20] Hinze JO. Turbulence. McGraw-Hill, New York 1975.

[21] Hussein HJ, Capp SP, George WK. Velocity Measurements in a High-ReynoldsNumber, Momentum-Conserving, Axisymmetric, Turbulent Jet. Journal of Fluid Mechanics, Vol. 258, 1994, pp. 31-75.

[22] Sundaram S, Collins LR. Numerical Considerations in Simulating a Turbulent Suspension of Finite-Volume Particles. Journal of Computational Physics, Vol. 124, 1996. pp. 337-350.

[23] Eaton JK. Local Distortion of Turbulence by Dispersed Particles. AIAA Fluid Dynamics Conference, Norfolk, VA, AIAA-99-3643, 1999 June. 


\section{Chapter 5 Potential Benefits of Hydraulic-Electric Generators}

\subsection{Introduction}

Wind turbine sizes have been steadily increasing over the last few decades due to advances in system design and component technology. The next generation of wind turbines to be employed in 2020 and beyond are likely to include extreme-scale systems (10 MW or more) for which the larger rotors have the potential to capture more energy per swept area due to higher wind speeds available at higher altitudes ${ }^{[1]}$. For extremescale systems, off-shore wind turbines hold promise of being an important source of energy due to a number of advantages ${ }^{[2-4]}$. Firstly, off-shore sitting allows high wind speed (which typically increases when distance from the shore goes further) and low turbulence and low wind shear (which can help to reduce fatigue failure and increase energy capture). Secondly, off-shore sitting may allow larger turbines and larger wind farms in uninhabited sea areas, where noise and visual impact to humans and wildlife can be minimized. However, off-shore locations must include expensive marine support structures and undersea electric transmission wires with coastal substations. In addition, variable weather conditions result in increased time for transportation and installation that add to overall capital costs. Furthermore, it is more complicated to integrate off-shore wind farms into electrical grids because the unsteady power output (as wind speed varies throughout the day) is difficult to make energy output uniform in time since it is impractical to include co-located off-shore gas turbines to provide offset power during periods of low wind. As a result, the cost of energy for off-shore wind energy is higher than many conventional energy sources and on-shore wind energy. 


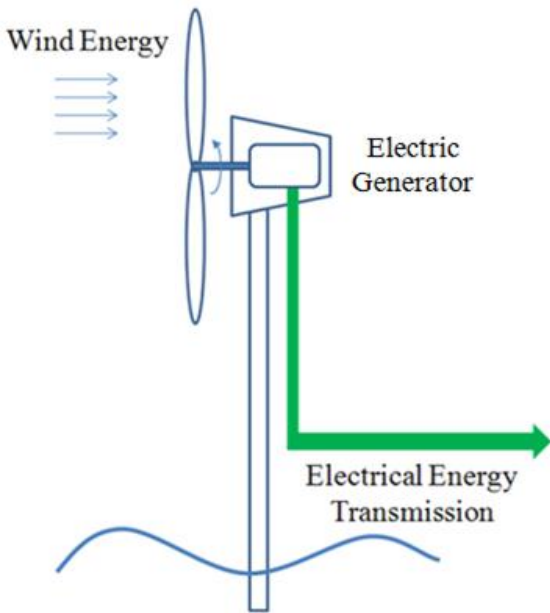

a)
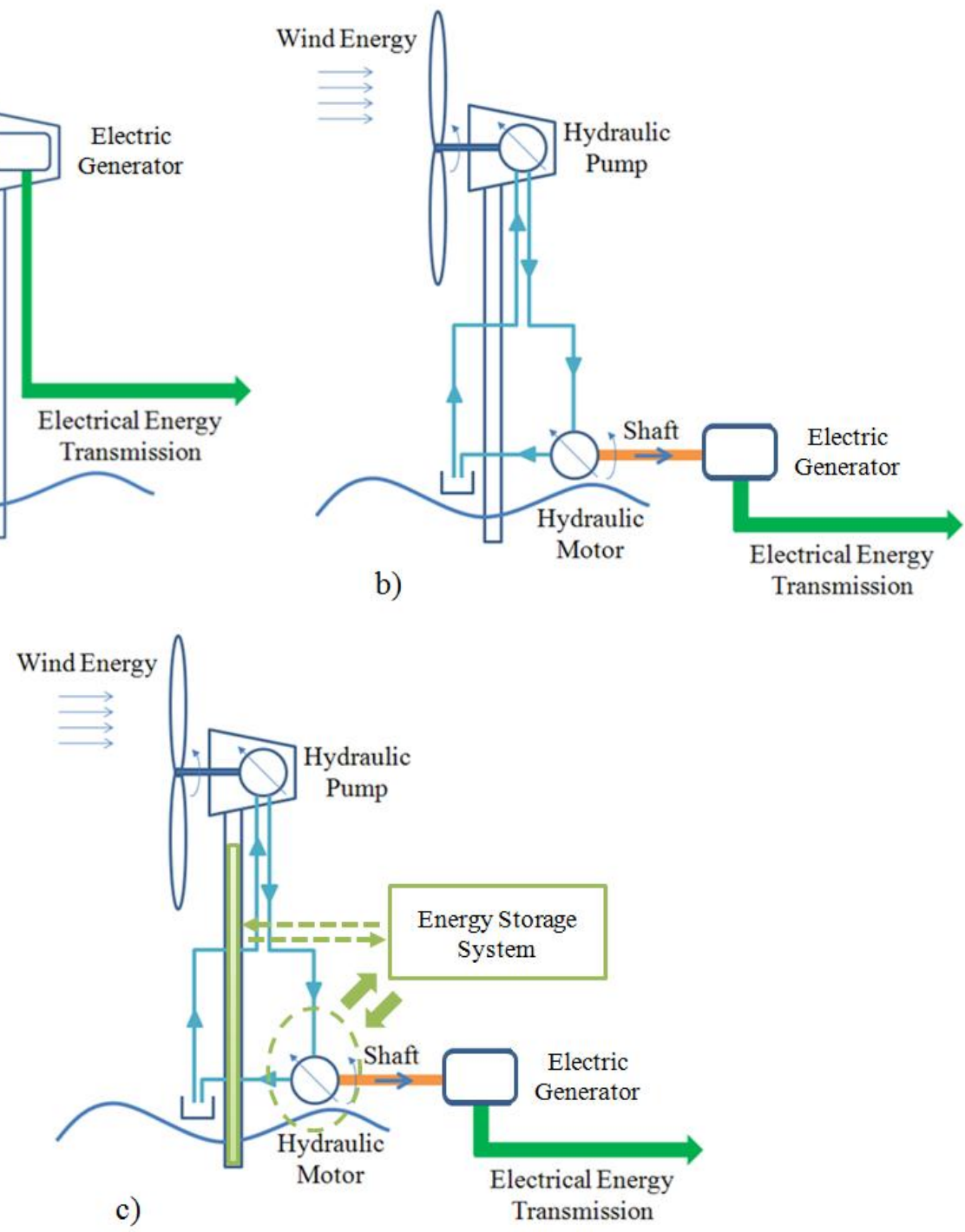

Fig. 5.1 Sketch of off-shore wind turbine system: a) conventional with electrical generator in nacelle on top, b) hydraulic-electric with electrical generator at base, and c) hydraulic-electric generator system with energy storage in tower

To reduce the cost of energy for extreme-scale wind turbines, especially those offshore, a modification of the power generation is proposed as shown in Fig. 5.1. In the conventional concept (Fig. 5.1a), the rotor shaft is converted to electrical power using an electrical generator. A gearbox is generally used to convert the low-speed rotor rpm to 
the higher rpm required for the electric generator to operate most efficiently. For extreme-scale systems, the rotor rpm is reduced even further because of increased blade radius combined with a limit on blade tip speed (ca. $80-100 \mathrm{~m} / \mathrm{s}$ ). The increased gear ratio may require multiple gearbox stages ${ }^{[1,2]}$ that increase complexity and probability of failure or may require more expensive and heavier direct-drive generators to eliminate the gearbox entirely (Ref. 5).

In the proposed power generator approach, shown in Fig. 5.1b, the electric generator is moved down to ground/sea level so that the shaft work from the rotor is instead fed into a hydraulic pump in the nacelle linked to a hydraulic motor at the tower base which can driven the electric generator. This modification reduces the mass and complexity of the nacelle drive components, and can eliminate the gearbox ${ }^{[6]}$. Also, the electric generator can be located at ground/sea level for ease of maintenance and can be driven at a high uniform rpm to help eliminate the complexity and cost of variable-speed generator components. Perhaps most importantly, the relocation of the electric generator allows the tower head weight to be substantially reduced, allowing the structural mass of the tower and its foundations to also be reduced.

The above hybrid hydraulic-electric generator concept can also facilitate the use of an energy storage system in the tower volume as shown in Fig. 5.1c. In particular, a Compressed Air Energy Storage (CAES) system as described in Ref. 7 is highly compatible with such a hybrid generator. Energy storage can allow a more levelized power input to the electrical generator so that the generator rating need not be sized on the rate rotor power (e.g. $5 \mathrm{MW}$ for the baseline configuration used herein) but the generation can instead be sized based on the average annualized rotor power (e.g. $2 \mathrm{MW}$ 
for a typical rotor subjected to wind variability). This reduction in the generator rating also can reduce the capacity of transmission lines ${ }^{[7]}$ To determine whether such a CAES concept is cost-effective in the tower, a quantitative analysis is needed to examine the amount of energy which can be safely stored in a turbine tower of conventional geometry (fixed diameter, thickness and height).

The objectives of this work are to quantify the head mass and tower mass savings that may be possible with this hydraulic-electric generator concept. These savings will be computed under the assumption that the frequency response of the tower is maintained to be constant so as to avoid problems with structural resonance and vibration caused by blade rotation. In addition, this study will investigate the feasibility of using this hybrid generator concept in conjunction with compressed fluid energy storage inside the tower and quantify the time of energy storage. This is the first study to quantify the head and tower mass savings associated with introduction of hybrid hydraulic-electric power generation. In addition, this is the first study to examine the characteristics of a compressed air energy system located within the tower volume and to examine the impact on tower design and quantify the potential storage capacity.

\subsection{Tower Structural Model}

To quantify the benefits of the proposed approach using a well-documented system, the National Renewable Energy Laboratory (NREL) off-shore $5 \mathrm{MW}^{[8]}$ was employed as the baseline configuration and its main components and dimensions are listed in Tab. 5.1. Wind turbine towers are structurally designed so that they are not easily resonated by blade rotation; therefore the natural frequencies are important characteristics of tower design and safety. Therefore, NREL employed finite-element 
codes called BModes and $\mathrm{ADAMS}^{[9]}$ to determine modes of this baseline wind turbine tower with a fixed connection at the base for two cases: 1) without head mass, and 2) with head mass (including rotor and all nacelle contents). In BModes, 50 finite elements are used, and in ADAMS, 99 tower parts are used. The resulting natural frequencies for various modes are listed in Tab. 5.2 where FA and SS indicate fore-aft modes and sideto-side modes respectively. Also listed are frequencies from GH Bladed, an industrial software package for wind turbine analysis, that were computed as part of the present investigation. Both ADAMs and GH Bladed have been demonstrated to provide high fidelity for predicting mode frequencies ${ }^{[10]}$ Compared to NREL's models, it can be found that Bladed performs well, despite that many inputs of Bladed were set to the default values, because some values of the baseline design were not available.

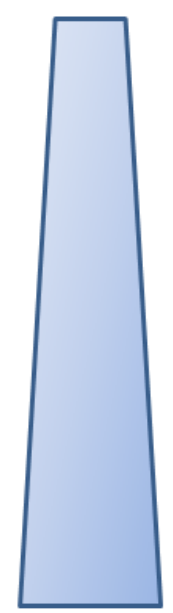

a)

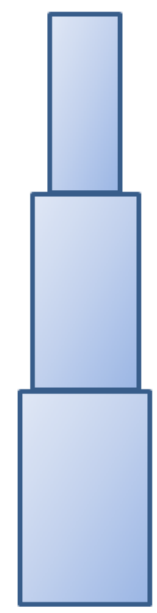

b)

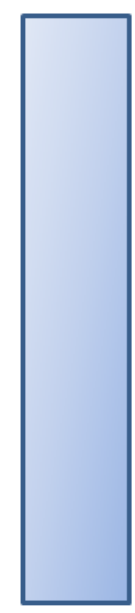

c)

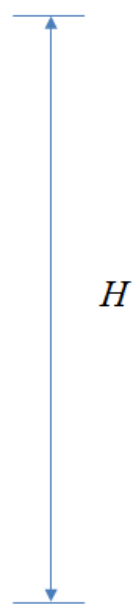

$H$
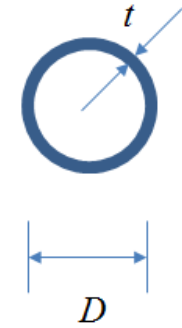

d)

Fig. 5.2 Tower shapes and dimensions: height $H$, diameter $d$, and wall thickness $t$

It is generally advantageous to employ a theoretical model for such analyses as this allows fundamental and direct correlations of input variables to performance 
characteristics. However, theoretical results require some system simplification and thus should be investigated to determine whether they are reasonable for the conditions of interest. The structural dynamics of a flexible wind turbine tower with a solid ground base can theoretically modeled as cantilever beam with a top mass. In Fig. 5.2, three shapes of wind turbine towers with the same height $H$ are shown: a) tapered shape which is the most realistic, b) stepped sections with discrete diameters which is used in most analytic codes, and c) constant diameter shape which is most used in theoretical analysis. In general, the tower cross section is circular as shown in Fig. 5.2d, with an outer diameter, $D$, and wall thickness, $t$. Theoretically, the first natural frequency for the latter shape can be calculated for this model without head mass:

$$
f_{1}=\sqrt{\frac{k_{f, e f f}}{M_{T}}}
$$

In this expression, $M_{T}$ is mass of tower and $k_{f, e f f}$ is the effective flexural stiffness with the form of

$$
\begin{aligned}
& k_{f, e f f}=\frac{2^{n_{1}} 3^{n_{2}} E I}{H^{3}} \\
& I \approx \frac{\pi D^{3} t}{8}
\end{aligned}
$$

In Eq. 5-2a , $E$ is Young's modulus of the tower material, $I$ is cross-section moment of inertia of the tower geometry, while $n_{1}$ and $n_{2}$ are two non-negative integers whose values are depended on load type. For example, $n_{1}=0$ and $n_{2}=1$ when bendingforce loaded, and $n_{1}=3$ and $n_{2}=0$ when distributed-weight loaded ${ }^{[11,12]}$. The theoretical 
expression of Eq. 5-1 is modified when a head mass $M_{H}$ is added on the top of the tower, so that the natural frequency becomes:

$$
\begin{gathered}
f_{1}=\sqrt{\frac{c k_{f, e f f}}{M_{T}+M_{H}}} \\
c=\frac{3 M_{T}}{3 M_{T}+8 M_{H}}
\end{gathered}
$$

The coefficient $c$ is based on relative force contribution and the head mass connection to the tower ${ }^{[11,12]}$.

Tab. 5.1 Characteristics and properties of the NREL $5 \mathrm{MW}$ off-shore wind turbine

\begin{tabular}{cc}
\hline Parameter & Value and units \\
Rating & $5 \mathrm{MW}$ \\
Rotor diameter & $126 \mathrm{~m}$ \\
Drivetrain & High speed multi-stage gearbox \\
Rotor mass & $110,000 \mathrm{~kg}$ \\
Nacelle mass & $240,000 \mathrm{~kg}$ \\
Tower height & $87.6 \mathrm{~m}$ \\
Tower diameter & base: $6 \mathrm{~m}, \mathrm{top}: 3.87 \mathrm{~m}$ \\
Tower thickness & base: $0.027 \mathrm{~m}, \mathrm{top}: 0.019 \mathrm{~m}$ \\
Tower mass & $347,460 \mathrm{~kg}$ \\
Tower Young's modulus & $210 \mathrm{GPa}$ \\
Tower shear modulus & $80.0 \mathrm{GPa}$ \\
\hline
\end{tabular}

The theoretical analysis of the NREL 5 MW system obtained herein assumes a uniform tubular shape in the theory using average tower diameter and thickness in Tab. 5.1 and can only be used to calculate the first modes. In addition, the fore-aft and side-to- 
side are the same when there is a head mass addition because the theory assumes homogeneous bending. The theory was validated with NREL's first mode results without head mass to determine the two natural numbers in Eq. 5-2a, as $n_{1}=5$ and $n_{2}=0$. The comparison with the computational models is shown in Tab. 5.2 for the case with no head mass, and the theory is found to be quite accurate in terms of the first modes. Using the same $n_{1}$ and $n_{2}$ values with Eq. 5-3 to consider the case with head mass, the theory shows remarkably reasonable agreement with other codes as shown again in Tab. 5.2, yielding about $3 \%$ differences. Since the primary objective is to provide a generalized model to predict tower mass saving (rather than tower mode details), this theoretical model is employed for the remainder of this study. The theoretical model also allows the results to be scaled up from $5 \mathrm{MW}$ to $20 \mathrm{MW}$, where the geometry is fixed but the size and all associated dimensions are increased.

Tab. 5.2 Primary mode tower frequencies (in $\mathrm{Hz}$ ) as computed by computational methods and by theoretical method for $1^{\text {st }}$ mode $\left(f_{1}\right)$ and $2^{\text {nd }}$ mode $\left(f_{2}\right)$

\begin{tabular}{|c|c|c|c|c|c|c|c|c|}
\hline \multirow{2}{*}{$\begin{array}{l}\text { Mode } \\
\text { type }\end{array}$} & \multicolumn{4}{|c|}{ Zero head mass } & \multicolumn{4}{|c|}{ Full head mass } \\
\hline & BModes & ADAMS & Bladed & Theory & BModes & ADAMS & Bladed & Theory \\
\hline $1^{\text {st }} \mathrm{SS}$ & 0.8913 & 0.8904 & 0.893 & 0.8789 & 0.3291 & 0.3188 & 0.327 & 0.3207 \\
\hline $1^{\text {st }} \mathrm{FA}$ & 0.8913 & 0.8904 & 0.893 & 0.8789 & 0.3324 & 0.3218 & 0.332 & 0.3207 \\
\hline $2^{\text {nd }} \mathrm{SS}$ & 4.3743 & 4.3437 & 4.128 & N/A & 1.8805 & 1.8820 & 1.795 & N/A \\
\hline $2^{\text {nd }} F A$ & 4.3743 & 4.3435 & 4.128 & N/A & 2.2432 & 2.2391 & 2.165 & N/A \\
\hline
\end{tabular}

\subsection{Impact of Hydraulics on Head and Tower Mass}

To calculate tower mass saving, the NREL 5-MW baseline is modified to the hydraulic-electric design as shown in Fig. 5.1b. Based on analysis in Chapter 3, the head weight could be reduced by replacing the electrical generator with a hydraulic pump and 
by reducing or eliminating the gear box. As shown in Tab. 5.3, the quantitative mass reductions are listed which include a 33\% or $50 \%$ head mass reduction depending on whether the gearbox is simplified or eliminated. Note that use of a direct drive system with single stage gearbox ${ }^{[5]}$ for the conventional system, would result in an even larger generator mass so that the next combination of the gearbox and generator mass is not expected to change significantly from the multi-stage gearbox defined by NREL (and as listed in Tab. 5.1).

Tab. 5.3 Head weight comparison between conventional (electrical generator) and hybrid (hydraulic-electrical generators) systems

\begin{tabular}{|c|c|c|}
\hline $5 \mathrm{MW}$ wind turbine & Conventional & Hydraulic-Electric \\
\hline Hydraulic motor weight & $\mathbf{0}$ & $6,000 \mathrm{~kg}$ \\
\hline Gearbox weight & $120,000 \mathrm{~kg}$ & $0 \mathrm{~kg}-60,000 \mathrm{~kg} *$ \\
\hline Generator weight (in nacelle) & $60,000 \mathrm{~kg}$ & $\mathbf{0}$ \\
\hline $\begin{array}{l}\text { Other nacelle/drive } \\
\text { components }\end{array}$ & $60,000 \mathrm{~kg}$ & $60,000 \mathrm{~kg}$ \\
\hline Rotor weight & $110,000 \mathrm{~kg}$ & $110,000 \mathrm{~kg}$ \\
\hline Total head weight & $350,000 \mathrm{~kg}$ & $176,000 \mathrm{~kg}-236,000 \mathrm{~kg}$ \\
\hline
\end{tabular}

* $0 \mathrm{~kg}$ assumes gearbox is eliminated entirely with a direct drive concept.

Figure 5.3 shows the potential tower mass savings for the NREL 5 MW rotor. The baseline tower condition (at zero tower mass savings) represents a design whose natural first mode frequency $\left(f_{1}\right)$ is $0.32 \mathrm{~Hz}$ (shown by solid black line). This frequency is 1.4 times larger than the rated rotor rotation frequency $f_{R}$ of $0.23 \mathrm{~Hz}$ (shown by dashed black line) calculated based on rotor diameter $D_{\text {rotor }}$, tip speed ratio $\lambda=7.5$, and average wind speed $v=12 \mathrm{~m} / \mathrm{s}$. The difference between these two frequencies represents a safety 
factor of 1.4 since lower wind speeds will result in lower rotor rotation frequencies, while rotor rpm is kept fixed for higher wind speeds (up until cut-out conditions). Maintaining this safety factor can avoids problems of the proximity of a natural frequency to a rotor frequency can lead to tower resonance.

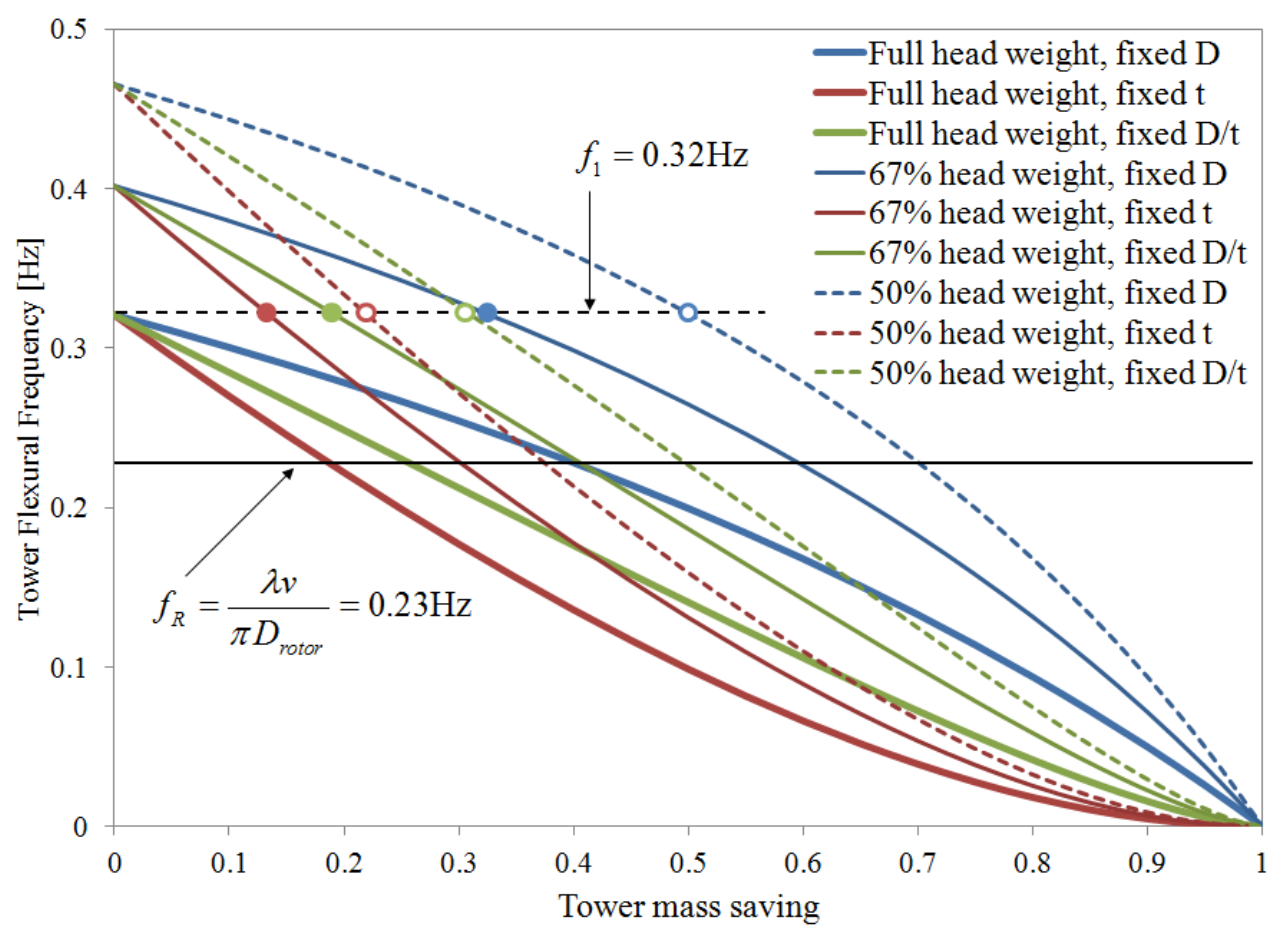

Fig. 5.3 The impact of tower mass savings (by reducing tower thickness and/or diameter) on tower frequency for three different head weights: i) baseline head weight described by thick solid lines, ii) head weight with $33 \%$ reduction described by thin solid lines and iii)

head weight with $50 \%$ reduction described by dashed lines. The solid and hollow symbols indicate the tower mass savings when maintaining the baseline tower natural frequency coupled with a 1.4 safety factor $\left(1.4 f_{1 \mathrm{p}}\right)$ for respective head weight reductions of $33 \%$ and $50 \%$. Note that the rotor rated frequency $\left(f_{R}\right)$ is given by black solid line

If one considering the new hydraulic-electric design with a head weight equal to $50 \%$ or $67 \%$ of the conventional head weight, the first mode frequency $\left(f_{1}\right)$ at zero tower mass savings is even higher at $0.40 \mathrm{~Hz}$ and $0.47 \mathrm{~Hz}$ respectively. This increase in 
frequency indicates that the baseline tower is over designed once the head mass is reduced, which provides a possibility to reduce the tower structure. This reduction can be achieved in various approaches: I) by reducing $D$ with a fixed $t$, II) by reducing $t$ with a fixed $D$, or III) reducing $D$ and $t$ with a fixed $D / t$. By making the low head mass tower have the same tower frequencies as the original case, the rotational frequency of the rotor and its third multiple are unchanged and so tower safety design is retained. As shown in Fig. 5.3, all three approaches show that an increased savings in tower mass (generally desirable) results in a reduction of tower frequency (generally undesirable), and the degree of sensitivity depends on which approach is employed. To determine the degree of mass savings that is reasonable to employ, one may assume that the new tower design should maintain the same 1.4 safety factor, i.e. should recover an $f_{1}$ of $0.32 \mathrm{~Hz}$ to be consistent with the original design structural constraints. Figure 5.3 shows that all three approaches yield a tower mass savings at this safety constraint (as shown by the hollow and solid symbols for a head weight of $50 \%$ or $67 \%$ of the conventional head weight). Also, it can be seen that fixing $D$ and reducing $t$ is the most effective approach to reducing tower mass while maintaining the 1.4 safety factor. If the head weight can be reduced by $50 \%$, this case leads to a tower mass savings of also about $50 \%$, which is very substantial. This same methodology is used to determine tower mass savings for extreme-scale turbine of up to $20 \mathrm{MW}$. It shows that there exists same potential for tower mass saving using the new hydraulic-electric design for extreme-scale systems. That means the cost savings costs for tower manufacture and transportation based on using the hydraulic-electric design can be dramatic for large wind turbines. 


\subsection{Impact of Tower Design on Tower Storage}

To calculate the potential energy storage capability in the tower volume of a wind turbine, the NREL 5-MW baseline is modified to include the hydraulic-electric design integrated with CAES as shown in Fig. 5.1c. For the hydraulic-electric/CAES combination, the open accumulator concept has been shown to be compatible for this combination $^{[13]}$, and its compressed air energy density $\left(E_{\eta}\right)$ can be computed as

$$
E_{\eta}=p_{\text {comp }}\left(\ln \frac{p_{\text {comp }}}{p_{a}}-1\right)+p_{a}
$$

In this expression, $p_{a}$ is the atmosphere pressure and $p_{\text {comp }}$ is the stored pressure. The above results show that energy density increases with storage pressure. However, increasing pressure also requires increased tower thickness since the inner volume now serves as a pressurized vessel. To determine the most efficient usage of tower thickness for energy storage requires consideration of safety limits for both fatigue and bursting. The fatigue and bursting pressure can be computed as

$$
\begin{aligned}
& p_{\text {fatigue }}=\frac{4 S_{u t}\left(t_{n}-t_{0}\right)}{D} \\
& p_{\text {bursting }}=\frac{4 S_{u t} t_{n}}{7 D}
\end{aligned}
$$

In this expression, $S_{u t}$ is the ultimate tensile strength, and subscript $n$ and 0 indicates the increased wall thickness $(t)$ and the original. For the $5 \mathrm{MW}$ baseline tower, these two safety constraints are shown as a function of storage pressure in Fig. 5.4 for three types of tower material: conventional steel (with an ultimate tensile strength of 636 
$\mathrm{MPa}$ ), carbon fiber E-Glass (with an ultimate tensile strength of $1770 \mathrm{MPa}$ ) and carbon fiber S-glass (with an ultimate tensile strength of $2358 \mathrm{MPa}$ ). It can be seen that low pressure systems are dominated by the fatigue constraint. However, very high pressures are dominated by the bursting constraint. The condition where these two constraints are equal is referred to as the crossover pressure and has been shown by previous studies of hydrogen storage in wind turbine towers to be the optimal in terms of cost benefits ${ }^{[14]}$ since it is the highest pressure for which the thickness requirements do not increase considerably. Therefore, the crossover pressure was also chosen as the design storage pressure for the present study as it requires only a moderate reinforcement of tower wall to store compressed air.

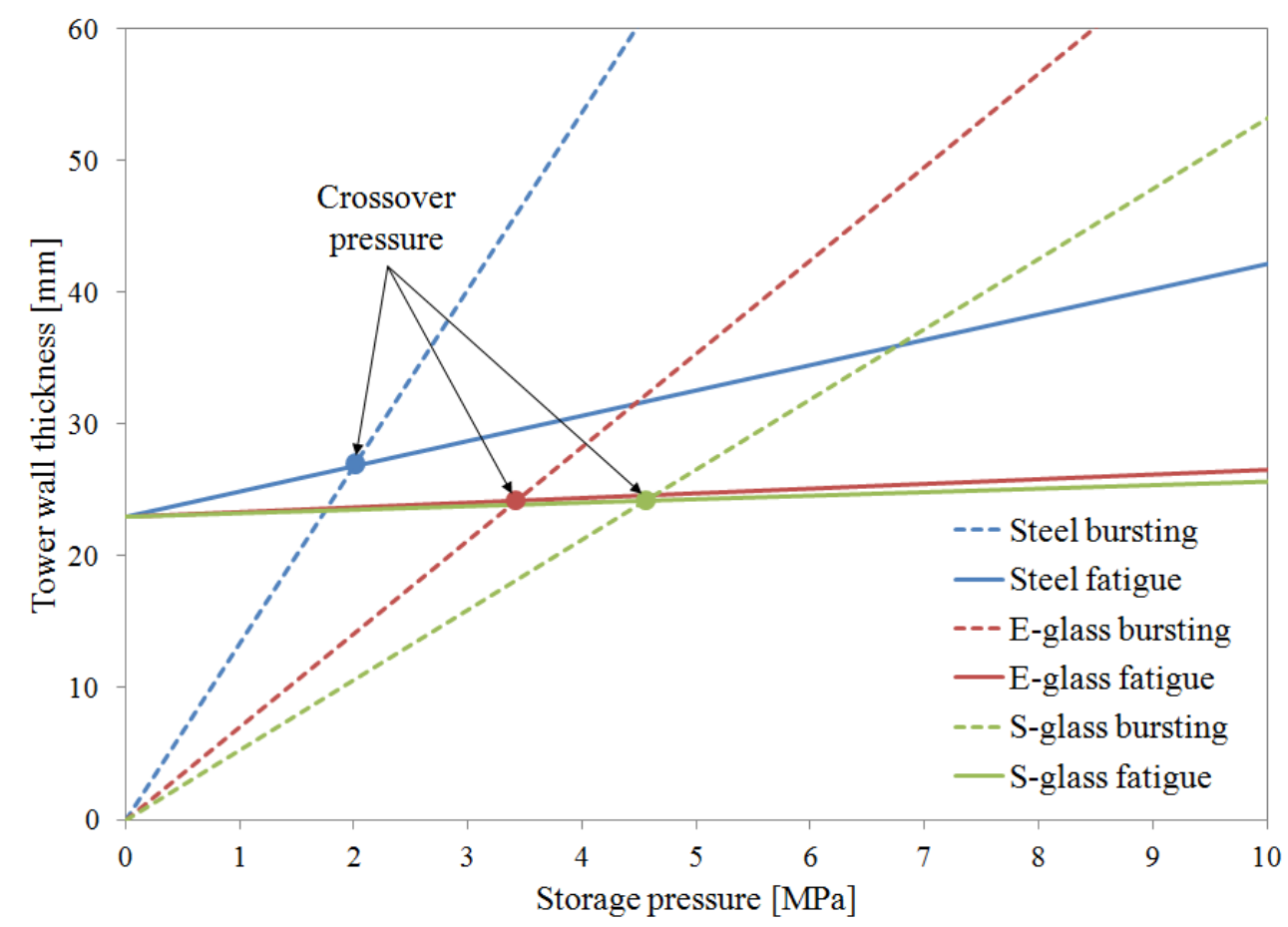

Fig. 5.4 Tower safety requirement if compressed air is stored inside a $5 \mathrm{MW}$ system 
Based on this assumption, the associated wall thickness for crossover is shown by a solid symbol and ranges from $36 \mathrm{~mm}$ for steel to $24 \mathrm{~mm}$ for S-Glass. It can be seen that S-Glass allows a significantly larger storage pressure than steel, without any increase in tower thickness. It also important to note that there is an approximate limitation in wall thickness based on to current manufacturing technology, whereby the maximum thickness of steel cannot exceed $4 \mathrm{~cm}$ while that for epoxy fiber cannot exceed $2 \mathrm{~cm}$. As shown by Fig. 5.4, these limitations do not pose a severe constraint for a $5 \mathrm{MW}$ system.

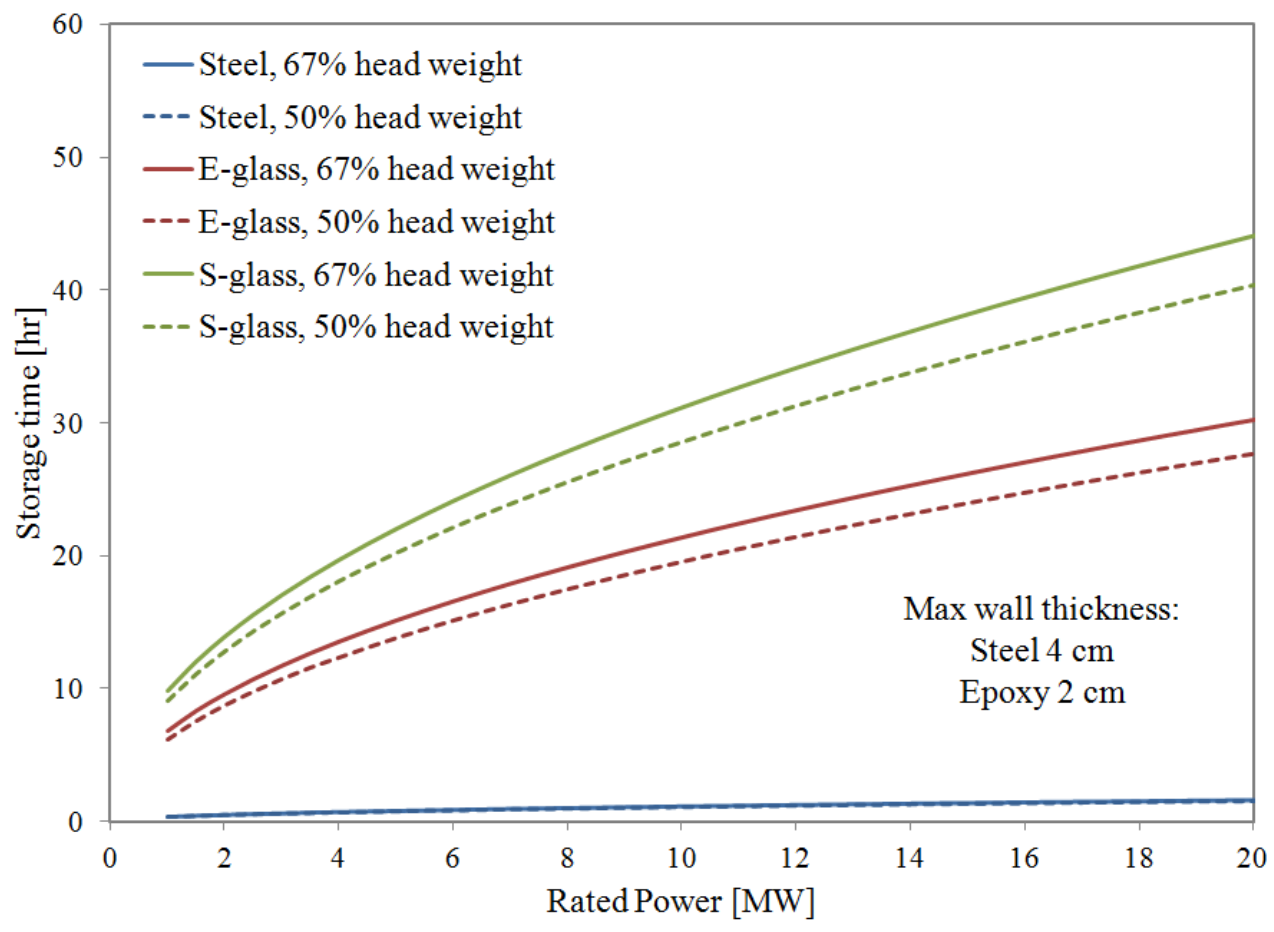

Fig. 5.5 Storage time for different rating power

Based on the crossover pressure criterion, the storage hours can be calculated for a specified wind turbine in terms of the above compressed air energy density. In 
particular, the potential storage time $(T)$ in the tower inner volume ( $V_{\text {inner }}$, based on inner tower diameter and height) can be expressed as

$$
T=\frac{E_{\eta} \cdot V_{\text {inner }}}{3600 P_{W T} \cdot C F}
$$

In this expression, $V_{\text {inner }}$ is tower inner volume based on inner diameter and height, $P_{W T}$ is wind turbine rated power, and $C F$ is capacity factor of average annual electrical power produced relative to rotor rated power. The capacity factor is assumed to be 0.3 consistent with previous studies ${ }^{[2]}$. The factor of 3600 is included in the denominator to convert the storage time from SI units of seconds to units of hours. Figure 5.5 shows the storage hours as a function of turbine power ratings based on Eqs. 5-4 and 5-6 for the three tower materials and for both the for a head weight of $50 \%$ or $67 \%$ of the conventional head weight. It is seen that the head weight mass does not impact the storage considerably, and that the storage time for the steel tower is negligible. This indicates that the compressed air energy storage within the tower volume for a steel fabricated tower is not practical. However, Fig. 5.5 shows that extreme-scale turbines with E-glass and S-glass can provide substantial energy storage times, on the order of 24 hours. This large increase in storage hours is partially due to the higher storage pressure but it is primarily due to the fiber glass tower (with a thickness of $2 \mathrm{~cm}$ ) having a diameter which more than doubled compared to the steel tower (with a thickness of $4 \mathrm{~cm}$ ). This change in diameter allowed the same structural frequency of the tower for both the steel and fiberglass new designs per safety constraints of the original tower. This storage capacity generally is enough to provide a smooth and constant electrical power supply ${ }^{[15]}$. This is a significant finding as it has been proposed that the carbon fiber may represent 
the next generation of tower material. Therefore, the tower volume should be considered as a storage vessel if a hydraulic-electric generator concept is employed. However, as with the results in Section 5.3, a much more detailed engineering and cost analysis is required to determine whether these concepts represent technologies can lead to a significant cost-of-energy savings. 


\section{Chapter 5 References}

[1] Musial W, Ram B. Large-Scale Offshore Wind Power in the United States: Assessment of Opportunities and Barriers. Technical Report NREL/TP-500-40745; 2010

[2] Henderson AR, Morgan C, Smith B, Sørensen HC, Barthelmie RJ, Bosemans B. Offshore Wind Energy in European - A Review of the State-of-the-Art. Wind Energy 2003; 6: 35-52

[3] Esteban MD, Diez JJ, López JS, Negro V. Why Offshore Wind Energy? Renewable Energy 2011; 36: 444-450

[4] Green R, Vasilakos N. The Economics of Offshore Wind. Energy Policy 2011; 39: 496-502

[5] Polinder H, van der Pijl FFA, de Vilder GJ, Tavner PJ. Comparison of Direct-Drive and Geared Generator Concepts for Wind Turbines. IEEE Transactions on Energy Conversion; 2006; 21(3): 725-733

[6] Polinder H, Bang D, van Rooij RPJOM, McDonald AS, Mueller MA. 10 MW Wind Turbine Direct-Drive Generator Design with Pitch or Active Speed Stall Control. Electric Machines \& Drives Conference, 2007. IEMDC '07. IEEE International (Vol.2); 2007

[7] Qin C, Loth E, Li P, Simon T, Van de Ven J, Crane SE, Pourmousa A. Spray-Cooling Concept for Wind-Based Compressed Air Energy Storage. 11th International Energy Conversation Engineering Conference (IECEC) 2013, San Jose, CA

[8] Jonkman J, Butterfield S, Musial W, Scott G. Definition of a 5-MW Reference Wind Turbine for Offshore System Development. Technical Report NREL/TP-500-38060; 2009

[9] Bir G, Jonkman J. Modal Dynamics of Large Wind Turbines with Different Support Structures. ASME $27^{\text {th }}$ International Conference on Offshore Mechanics and Arctic Engineering. NREL/CP-500-43045: Portugal; 2008

[10] Jeong MS, Cha MC, Kim SW, Lee I, Kim T. Effects of torsional degree of freedom, geometric nonlinearity, and gravity on aeroelastic behavior of large-scale horizontal axis wind turbine blades under varying wind speed conditions. Journal of Renewable and Sustainable Energy 2014; 6

[11] Warburton GB. The Dynamical Behaviour of Structures. New York: Pergamon Press; 1964 
[12] Rivin EI. Stiffness and Damping in Mechanical Design. New York: Marcel Dekker, Inc.; 1999

[13] Li P, Van de Ven J, Sancken C. Open Accumulator Concept for Compact Fluid Power Energy Storage. ASME 2007 International Mechanical Engineering Congress and R\&D Exposition (IMECE2007-42580); 2007

[14] Kottenstette R, Cotrell J. Hydrogen Storage in Wind Turbine Tower. Technical Report NREL/TP-500-34656; 2003

[15] Saadat M, Li PY. Modeling and Control of a Novel Compressed Air Energy Storage System for Offshore Wind Turbine. 2012 American Control Conference; 2012, Montreal, Canada 


\section{Chapter 6 Simulation of Spray Direct Injection Compression}

\subsection{Introduction}

Compressed Air Energy Storage (CAES) can bring a number of advantages when integrated to a power supply system with a variable and unsteady energy source. Taking wind energy as an example, off-peak or excess electricity can be used to drive compressors to pressurize ambient air, which is then stored in an accumulator. When user's electricity demands are high but the wind is not strong, the compressed air is released. The stored energy is extracted by burning the compressed air with fuel and expanding through a turbine to produce shaft work which can be converted to electrical energy through a generator. Thus, a smooth electrical output can be supplied to the grid despite the variability of the wind energy. However, this wind-turbine/gas-turbine CAES system can be efficient during adiabatic compression, because the built-up of thermal energy during this process is later dissipated to the surrounding atmosphere before it can be utilized due to long-time storage of several hours ${ }^{[1]}$. Additionally, this windturbine/gas-turbine CAES system becomes problematic for off-shore wind turbines, because these gas turbine and fuel are difficult to locate and operate off-shore. Considering the trend to off-shore wind turbines in recent wind energy development, a new type of CAES technology is needed to gain the advantages of energy storage. In Ref. 2, a novel approach to compressed air energy storage was proposed for off-shore wind turbines based on a liquid piston for a compressor and expander combined with an open accumulator for storage. Unlike traditional piston, a liquid piston uses a liquid column on top of the piston surface to compress gas and this allows a shaped chamber for the portion above the mechanical piston stroke. In particular, the top part of the cylinder need not be 
a cylinder but can be a tapered cone to help minimize dead volume, decrease seal leakage

and increase overall pressure ratio. Based on this liquid piston concept, Qin and Loth ${ }^{[3]}$ modeled a spray enhanced liquid piston compression to allow near isothermal compression (to avoid wasteful adiabatic compression). This is achieved through droplet heat transfer with a large total surface area and this conceptual study showed that a highefficiency compression process could be established with a mass loading of unity and small droplets of about 30 microns in diameter. In another study, the proposed CAES system with spray-cooled liquid pistons and multiple stages for compression was simulated for a 5-MW off-shore wind turbine baseline ${ }^{[4]}$. These one-dimensional simulation results showed that spray cooling may effectively improve the overall performance of energy storage system, which can bring benefits to off-shore wind turbine systems.

However, the above investigations were limited to one-dimensional laminar flow simulations, and it was recommended that two- or three-dimensional effects with turbulence should be considered and included in simulations. These effects, for example turbulent kinetic energy, cylinder vortices, and radial gradients of temperature and droplet concentration can affect the net heat transfer and degree of compression efficiency and overall performance. In the present chapter, multi-phase computational fluid dynamic (CFD) are implemented in an axisymmetric domain with turbulent flow predictions. These simulations can help understand the impact of droplet dispersion and spray cooling in liquid piston compression and allow an understanding of the predictive performance of the previous one-dimensional simulation results. This understanding can 
provide guidance in preliminary piston design or linear control system design, when simply using 1D model within acceptable tolerance.

The objective of this work is to employ an axisymmetric Reynolds-Averaged Navier-Stokes approach to simulate the compression portion of a single cycle for a liquid piston with spray cooling. The geometry and flow field associated with intake and exhaust valves were not included since they would require a three-dimensional approach due to their off-center location at the chamber head. Similarly, a single spray nozzle was placed asymmetrically to also avoid need for three-dimensional approach associated with side-wall spray injection. To the author's knowledge, the present chapter is the first to simulate spray-enhanced liquid piston compression two-dimensionally and first to investigate effects of flow turbulence, vortex formation and radial non-uniformity.

\subsection{Methodology}

\subsubsection{Numerical methods}

In the present chapter, an Eulerian numerical approach is chosen for the gas phase and the Lagrangian approach for the liquid phase. To ensure both numerical stability and low computational cost, the continuous phase (ambient gas) is solved by Reynoldsaveraged Navier-Stokes (RANS) equations, using the standard k- $\varepsilon$ model, while the dispersed phase (droplet) is solved by trajectory discretization with a discontinuous random walk (DRW) model to produce synthetic turbulent fluctuations.

The gas-phase is treated with a standard k- $\varepsilon$ model ${ }^{[5]}$ whereby the turbulent kinetic energy $(k)$ and its dissipation rate $(\varepsilon)$ are solved in two transport equations: 


$$
\begin{aligned}
& \frac{\partial}{\partial t}\left(\rho_{g} k\right)+\frac{\partial}{\partial x_{i}}\left(\rho_{g} k u_{i}\right)=\frac{\partial}{\partial x_{j}}\left[\left(\mu_{g}+\frac{\mu_{t}}{\sigma_{k}}\right) \frac{\partial k}{\partial x_{j}}\right]+G_{k}+G_{b}-\rho_{g} \varepsilon-Y_{M} \\
& \frac{\partial}{\partial t}\left(\rho_{g} \varepsilon\right)+\frac{\partial}{\partial x_{i}}\left(\rho_{g} \varepsilon u_{i}\right)=\frac{\partial}{\partial x_{j}}\left[\left(\mu_{g}+\frac{\mu_{t}}{\sigma_{k}}\right) \frac{\partial \varepsilon}{\partial x_{j}}\right]+C_{1 \varepsilon} \frac{\varepsilon}{k}\left(G_{k}+C_{3 \varepsilon} G_{b}\right)-C_{2 \varepsilon} \rho_{g} \frac{\varepsilon^{2}}{k}
\end{aligned}
$$

In equation (6-1) and (6-2), $u_{i}$ refers to gas velocity in tensor notation, $x_{i}$ refers to the Eulerian coordinates in tensor notation, $\rho$ is density, and the subscript $g$ indicates a gas-phase property. Herein, the constants and non-dimensional numbers have the following values: $C_{1 \varepsilon}=1.44, C_{2 \varepsilon}=1.92, C_{\mu}=0.09, \sigma_{k}=1.0, \sigma_{\varepsilon}=1.3$. In the above expressions, turbulent viscosity $\left(\mu_{t}\right)$ is calculated by:

$$
\mu_{t}=C_{\mu} \rho_{g} \frac{k^{2}}{\varepsilon}
$$

The turbulent kinetic energy generation and distraction terms $\left(G_{k}, G_{b}\right.$, and $\left.Y_{M}\right)$ are described in Ref. 5. In this model, turbulent two-way coupling is not considered so that none of these terms are related to the presence of the droplets. Instead the two-way coupling between continuous phase and discrete phase is included through interphase interaction forces as a sink term for the mean gas momentum and interphase heat transfer as a sink term for the mean gas temperature.

To describe the trajectory of a discrete phase particle (droplet), the equation of motion for droplets with centroid velocity $v_{i}$ is expressed in a Lagrangian reference frame [6]. 


$$
\frac{d v_{i}}{d t}=F_{D}\left(u_{i}-v_{i}\right)+\frac{\left(\rho_{d}-\rho_{g}\right)}{\rho_{d}} g_{i}
$$

In this expression, subscript $d$ indicates a droplet property and the forces applied to the droplet are assumed to be dominated by drag and gravity forces (which is reasonable given the high ratio of droplet density to gas density. The first term on the right hand side is drag force per particle mass per relative velocity and $F_{D}$ is defined as

$$
F_{D}=\frac{3 \mu_{g} C_{D} \operatorname{Re}}{4 \rho_{d} d^{2}}
$$

In this expression, $\mathrm{Re}$ is the drop aerodynamic Reynolds number defined along with the magnitude of relative velocity $(w)$ and the component of relative velocity in the $i$ direction $\left(w_{i}\right)$ as.

$$
\begin{aligned}
& \operatorname{Re} \equiv \frac{\rho_{g} d w}{\mu_{g}} \\
& w=\sqrt{w_{i} w_{i}} \\
& w_{i}=v_{i}-u_{i}
\end{aligned}
$$

A spherical droplet assumption is reasonable for Weber number less than unity ${ }^{[6]}$, and this criterion is appropriate for the droplets considered herein based on their size and relative. Thus, the drag coefficient $C_{D}$ for spheres is used and taken from Morsi and Alexander ${ }^{[7]}$.

The gas velocity in Eqs. 6-4 and 6-6 (needed for droplet instantaneous trajectories) can be expressed in terms of mean and fluctuation gas velocity values: 


$$
u_{i}=\overline{u_{i}}+u_{i}^{\prime}
$$

The mean value (first term on RHS) is obtained from RANS solution. The fluctuation velocity is also important to include to properly capturing turbulent diffusion. To obtain this term synthetically from the gas turbulent kinetic energy and dissipation, the discrete random walk (DRW) model of Ref. 8 is employed. The DRW model is achieved with a random number $(\sigma)$, and an average fluctuation strength $\left(u_{r m s}^{\prime}\right)$. In particular, an instantaneous gas velocity fluctuation is held constant over an interaction time and based on random sampling for each directional component such that the net variation over many samples is consistent with the gas turbulent kinetic energy

$$
\begin{aligned}
& u_{i}^{\prime}=u_{r m s}^{\prime} \sigma_{i} \\
& u_{r m s}^{\prime}=\left(\frac{2}{3} k\right)^{1 / 2}
\end{aligned}
$$

In this expression, $\sigma$ represents three independent Gaussian random numbers whose mean value is zero but whose rms value is unity. A new random number is generated along the droplet path when the integration time along the path exceeds the droplet-eddy interaction time $\left(\tau_{\text {int }}\right)$. This interaction time is based on the minimum of the overall eddy lifetime $\left(\tau_{\Lambda}\right)$ and the eddy traversal time $\left(\tau_{\text {tra }}\right)$ which are defined below as

$$
\begin{gathered}
\tau_{\Lambda}=C_{\tau} \frac{k}{\varepsilon} \\
\tau_{t r a, i}=\frac{\Lambda_{i}}{w}
\end{gathered}
$$


In these expressions, $C_{\tau}$ is the eddy timescale coefficient (whose calibrated value is 0.21 ) and $\Lambda$ is turbulence integral length scale (which is related to $k$ and $\varepsilon)^{[8]}$.

This present Eulerian-Lagrangian approach has been carefully modeled and validated with experiments and the results of mean axial velocity of both gas and droplets and droplet radial mass flux distribution indicated a good agreement in previous chapters.

For convective heat transfer between continuous and disperse phases, heat balance of the droplet can be expressed to relate particle temperature to surrounding gas temperature:

$$
\frac{\pi}{6} \rho_{d} d^{3} c_{d} \frac{d\left(T_{d}\right)}{d t}=\mathrm{Nu} \cdot k_{g} \pi d\left(T_{g}-T_{d}\right)
$$

where $c_{d}$ is the specific heat capacity of the particle, $k_{g}$ is the thermal conductivity of the gas, $T_{g}$ and $T_{d}$ are the local gas and particle temperatures respectively, and $\mathrm{Nu}$ is the Nusselt number. The Nusselt number can be obtained from the well validated RanzMarshall relationship as function of the Prandtl number $(\mathrm{Pr})$ and Reynolds number:

$$
\mathrm{Nu}=2+0.6 \operatorname{Re}^{1 / 2} \operatorname{Pr}^{1 / 3}
$$

The Prandtl number can be written in terms of the specific heat capacity of the gas at constant pressure $\left(c_{p}\right)$ and the gas viscosity and conductivity as

$$
\operatorname{Pr}=\frac{c_{p} \mu_{g}}{k_{g}}
$$

This heat transfer formulation was recently validated with experimental data for spray-cooling in a channel flow ${ }^{[3]}$. For the present chapter, droplet mass transfer is 
neglected since the large amount of liquid discharging in the cylinder establishes a near $100 \%$ humidity condition inside compression chamber.

Based on above description, the numerical approach can be employed to simulate spray-enhanced liquid piston compression with direct injection (via nozzle spraying within the cylinder). In the next section, several performance parameters are described which can quantify the effectiveness of the compression process.

\subsubsection{Performance parameters}

The amount of water injected can be expressed non-dimensionally in terms of the injected mass loading, defined as the ratio of water spray mass discharged $\left(m_{\text {spray }}\right)$ to the mass of dry air drawn into the piston chamber $\left(m_{a}\right)$, i.e.

$$
M L=\frac{m_{\text {spray }}}{m_{a}}
$$

The injected mass loading (ML) is calculated by using total discharged spray mass in a compression cycle. Injected mass loading is based on the liquid flow rate of a given nozzle, the injection period, and the compressed gas mass stemming from the intake pressure and initial compression chamber volume. In addition, one may define the transient mass loading $\left(\mathrm{ML}_{\mathrm{t}}\right)$ based on the instantaneous spray mass aloft in gas, which is therefore a function of time and during the compression cycle. Transient mass loading takes into account droplet losses caused by hitting the chamber wall or piston surface (and thus $\mathrm{ML}_{\mathrm{t}}<\mathrm{ML}$ for any droplet losses). These droplet losses may be counteracted by internal vortices that can help keep the droplets aloft in the gas for a longer time. 
During compression, one may defined the polytrophic index $(n)$ as the relationship between pressure increase and volume decrease

$$
\frac{p_{2}}{p_{1}}=\left(\frac{V_{1}}{V_{2}}\right)^{n}
$$

In the ideal case of isothermal compression (where work is used exclusively to increase pressure and no work is lost to internal energy rise), the temperature is constant. As a result, the pressure is linearly proportional to density (and inversely proportional to volume) during compression so that this index is unity. This ideal condition will result if there is high heat transfer between the droplets and the gas. In the non-ideal case of adiabatic compression, this index will equal the gas specific heat ratio $\left(\gamma_{g}\right)$, whose value for air is 1.4. This condition will result in the least amount of work directed at pressure rise since and the largest temperature rise.

While a perfectly ideal case of isothermal compression is impossible, one may consider the limiting case of infinitely small drops so that the heat transfer is infinitely fast. In this limit, the polytrophic index can be expressed in terms of the on liquid spray mass loading as

$$
n=\gamma_{g} \frac{1+M L \cdot\left(c_{d} / c_{p}\right)}{1+\gamma_{g} M L \cdot\left(c_{d} / c_{p}\right)}
$$

This expression for the polytrophic index represents the smallest possible value for a given droplet mass loading only ${ }^{[9]}$. When the mass loading becomes infinite, the polytrophic index trends to one, which indicates isothermal compression. Thus, 
theoretically, discharging large amount of liquid in small droplets (to promote fast heat transfer) helps to achieve near-isothermal compression.

If pressure ratio and volume ratio are known for a certain compression process, the average polytrophic index can be calculated, which represents the overall performance of the compression. However, the polytrophic index for the actual compression process will vary with time depending on the instantaneous heat transfer rate and the transient mass loading. The entire compression process can be discredited into a number of sub-processes, where polytrophic indexes can be assumed as constants.

To further quantify the performance of a spray-cooling during the compression, "compression efficiency" is defined, by first considering the work required to compress the gas and the energy obtained for later expansion. This compression is based on pressure work and can be written in either integral form or (approximately) in discrete summation form as

$$
W=\int\left(p-p_{a t m}\right) d V \approx \sum\left(p-p_{a t m}\right) \Delta V
$$

The right-hand-side expression assuming a constant gas pressure between each interval (taken here as the average) with $\Delta \mathrm{V}$ is the volume change in a time interval. If the time-step is sufficiently small, this discrete form will converge to the integral definition. Within liquid piston compression process, air is compressed to a desired pressure, and then the compressed air exhausts out of the chamber. Note that this compression work includes not only that for compressing the air to the desired pressure but also that for pushing out the compressed gas during the exhaust process. So the 
volume changes in the discrete and integral form varies from the maximum (the intake volume at bottom dead center) to zero (needed to include the exhaust process).

Ideally, the air temperature is held constant due to droplet heat transfer so that the compression is isothermal. However, the overall compression will always be less than ideal due to many practical limitations. The compression efficiency of a given process is defined based on the difference to isothermal compression, where gas is compressed from the same initial pressure to the same final pressure of the given process:

$$
\eta=\frac{W_{i s o}}{W} \times 100 \%
$$

This result shows that isothermal compression (assuming an ideal gas) is the most efficient process as it yields an efficiency of 100\%. For spray-enhanced liquid piston compression, the objective is to reach as high of efficiency as possible.

\subsection{Test Conditions and Computational Domain}

The test conditions investigated are consistent for a single liquid piston chamber with geometry typical of conventional piston cylinders. In particular, a cylinder compression chamber with an inner diameter of $15 \mathrm{~cm}$, a length of $30 \mathrm{~cm}$, and a pressure ratio of 10 is employed. A multi-megawatt wind turbine would employ hundreds of these chambers for multi-stage compression.

For the present chapter, a single pressure-swirl nozzle orifice is located in the center of piston top wall. For spray discharge, pressure-swirl nozzle was found to be

most effective for liquid piston compression ${ }^{[4]}$. Based on previous studies to simulate this type of nozzle in Chapter 4, three droplet diameters were used (small, medium, and 
large) to represent the polydisperse size distribution. Each of the three diameters represents a bin so spherical drops where the small diameter is $7.5 \mu \mathrm{m}$, medium diameter is $25 \mu \mathrm{m}$, and large diameter is $55 \mu \mathrm{m}$. The injected mass fraction associated with the three bins for this type of spray nozzle was measured to be $2 \%$ for the small diameter, $57 \%$ for the medium diameter and $41 \%$ for the large diameter.

Herein, three different cases are considered for operational performance based on varying the intake/initial pressure and the spray mass injected. Case 1 is a first stage cylinder with an initial air pressure of one atmosphere and the same nozzle conditions as Ref. 3 which corresponds to a total injected mass loading of 0.12 . Case 2 is also in a first stage cylinder but the spray flow rate is adjusted to achieve the maximum experimental flow rate which allows droplets with the same mean diameter as Case $1^{[10]}$, which resulted in an injected mass loading of 1.6. Case 3 uses the same spray conditions as Case 2 but for a second stage cylinder with an initial air pressure of 9.6 atmospheres, which resulted in an injected mass loading of 0.185 (due to higher density and mass of air in the cylinder). Therefore, comparing Case 1 and 2 allows the effect of mass loading to be understood for a fixed initial pressure, while comparing Cases 2 and 3 allows the effect of initial pressure to be understood for a fixed injected liquid mass. In addition, comparing Cases 1 and 3 allows the effect of initial pressure to be understood for a similar mass loading.

In present chapter, CFD simulations are implemented in an axisymmetric domain as shown in Fig. 6.1a. Droplets are injected at $9 \mathrm{~mm}$ downstream of the nozzle orifice with specified velocity and mass flow rate based on experimentally obtained profiles. For all the cases, injected droplet temperature is $300 \mathrm{~K}$ and injected droplet Sauter mean 
diameter (SMD) is $30.6 \mu \mathrm{m}$. In first stage cylinder, initial air temperature is $300 \mathrm{~K}$ and initial air pressure is $101.325 \mathrm{kPa}$. In second stage cylinder, initial air temperature and pressure are based on the final temperature and pressure of case $2\left(1^{\text {st }}\right.$ stage outlet $)$ but with $10 \%$ decrease. The sidewall and liquid piston boundaries are assumed to be adiabatic and when a droplet impacts these walls, the droplet is removed the domain.

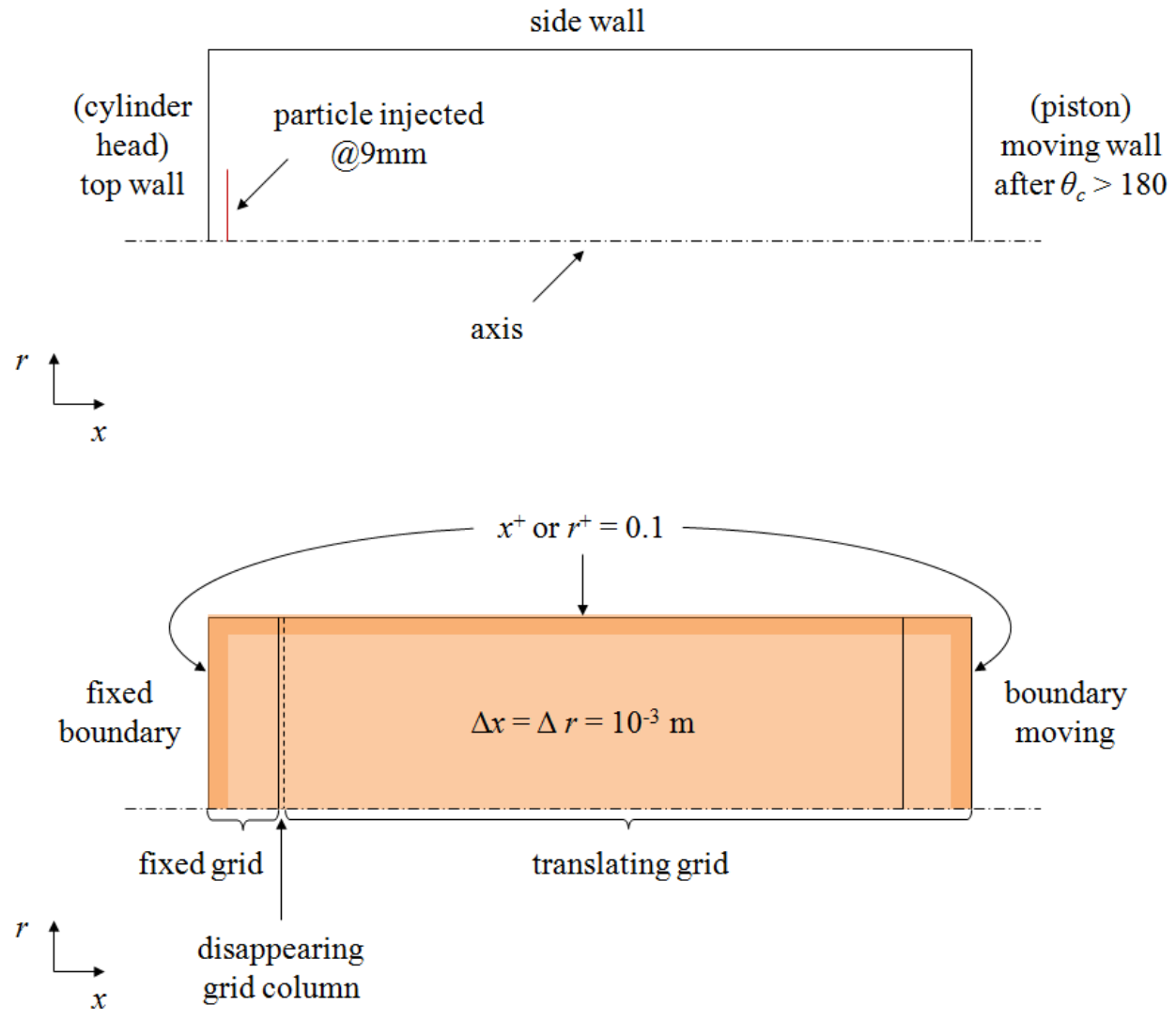

Fig. 6.1 Sketch of a) axisymmetric simulation domain, and b) grid resolution and dynamic mesh

To describe the dynamics of the piston surface a full compression cycle is considered, where the crank angle goes from 0 to 360 degrees. For the first half of the cycle, there is no expansion stroke (so that the complexities of intake valves can be 
ignored) and instead the piston is simply kept fixed at the bottom dead center. Droplet injection begins at 0 -degrees crank angle and continues to 180 degrees to allow spray to enter the chamber before compression. At 180-degree, the piston starts to moving upward to compress the air in the cylinder. Herein, the liquid piston surface can be described for the compression period using crank angle $\left(\theta_{c}\right)$ as:

$$
x=L+\frac{A}{2}\left(1-\cos \theta_{c}\right)-\sqrt{L^{2}-\frac{A^{2}}{4} \sin ^{2} \theta_{c}} \quad \text { for } \theta_{c}>180^{\circ}
$$

where $A$ is piston stroke, and $L$ is connecting rod length. For simplicity a cylindrical shape is assumed with $A=30 \mathrm{~cm}$ and $L=50 \mathrm{~cm}$. Similarly, the inner volume of chamber can be described as a function of crank angle. The piston surface for compression is driven by a crank operating at $60 \mathrm{rpm}$, which yields a cycle frequency of $1 \mathrm{~Hz}$ or a time of $0.5 \mathrm{sec}$ for $\theta_{c}$ to go from $180^{\circ}$ to $360^{\circ}$. This slow frequency is used to avoid liquid interface instabilities ${ }^{[3]}$ but as a side benefit it allows significant time for heat transfer to occur during compression. When the mass-averaged absolute pressure reaches the prescribed value (of ten times the initial pressure), the simulation stops. The rest of cycle is not simulated to avoid complexity associated with the outlet valve and exhaust process which would require a three-dimensional grid.

Based on this information, the half-stroke Reynolds number of the flow (based on cylinder diameter as well as gas density and piston surface velocity at $270^{\circ}$ ) is about $1.8 \times 10^{4}$, so the flow is generally turbulent. To satisfy both numerical accuracy and computational low cost, the structured grid during the inflow period $\left(0^{\circ}<\theta_{c}<180^{\circ}\right)$ was static and included a resolution of $10^{-3} \mathrm{~m}$ in the interior and a smaller grid spacing near wall the wall based on inner units $\left(x^{+}\right.$or $\left.r^{+}=0.1\right)$. For time integration a crank angle step 
of 0.1 degrees was chosen. The piston surface movement for the compression period $\left(180^{\circ}<\theta_{c}<360^{\circ}\right)$ must be handled computationally with care. As shown in Fig. 6.1b, a dynamic mesh model is included as piston surface moves to the left. The structured grid is adjusted as a function of time by translating the interior grid points in the axial direction with uniform size, except for the all the left-most column of cells labeled as the "disappearing grid column" in Fig. 6.1b). This column of interior grid points will be fixed on the left side but will translate on the right side yielding continuous compression until this difference between these two edged becomes less than $5 \%$ of its original grid size. At this point, the cell is merged with the cell on the right.

For comparison, one-dimensional laminar flow simulations based on the previous methodology ${ }^{[3]}$ were completed for the three cases, using the same mass loadings and a uniform droplet diameter of $30 \mu \mathrm{m}$ coupled with an injection velocity of $2 \mathrm{~m} / \mathrm{s}$. Otherwise, the initial pressure and temperature conditions are set to as same as the towdimensional simulations described above.

\subsection{Results and Discussions}

The present 2D simulations, the results can help to understand how droplet injection affects the performance of liquid piston compression. To study dynamics of different sizes of particles, Fig. 6.2 shows instantaneous positions of different size droplets. Small and medium sized droplets tend to follow the vortex, while large droplets trend to move along their trajectories with strong radial trajectories. However the combinations of the turbulent fluctuations and vortex cause the small and medium sized

particles hit the sidewall first. In the first stage, particles are generally fill the chamber by fulfill the chamber. But in the second stage, particles cannot reach the piston surface 
because of high density of gas. Thus, turbulence is important to understand droplet spatial distribution and small droplet losses, especially at the side wall.
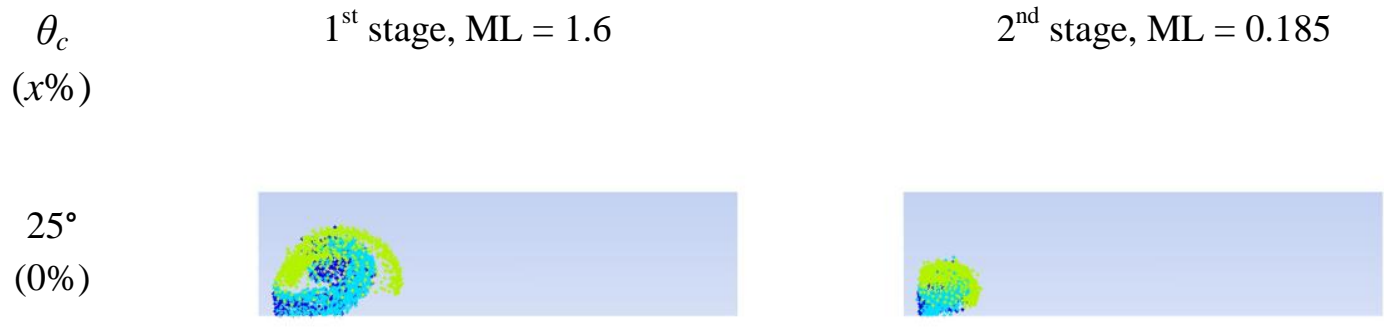

$45^{\circ}$

$(0 \%)$
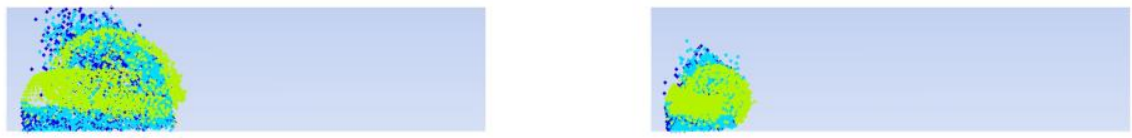

$90^{\circ}$

$(0 \%)$
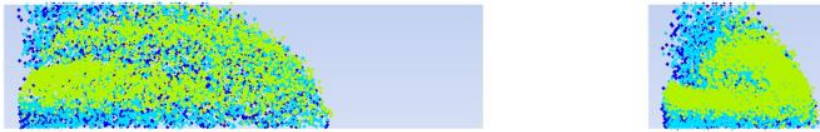

$180^{\circ}$

$(0 \%)$
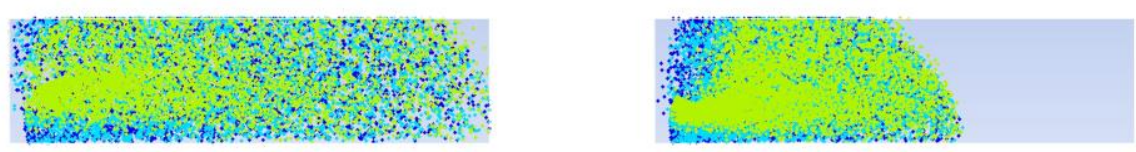

$270^{\circ}$

$(42 \%)$
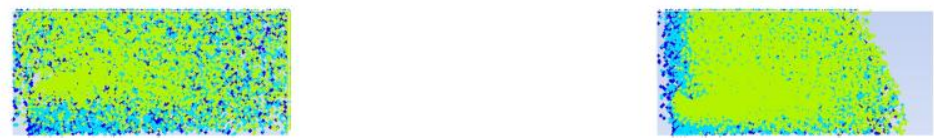

$320^{\circ}$

$(85 \%)$
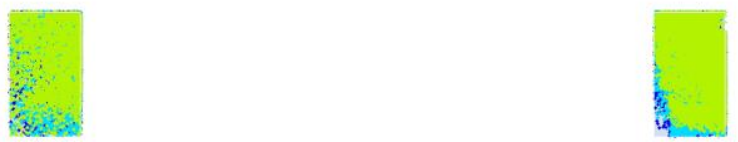

Fig. 6.2 Droplet position and size [large • ; medium • ; small • ] 


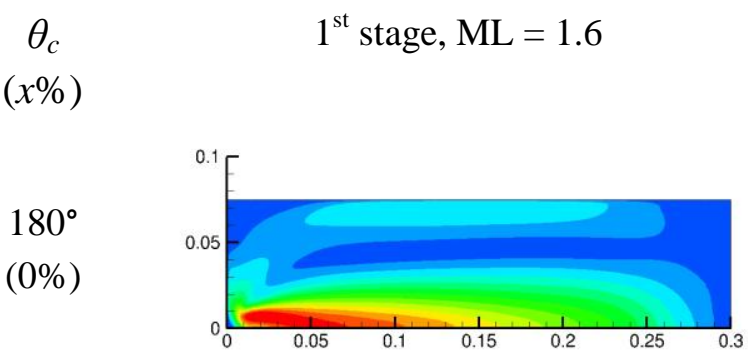

$$
2^{\text {nd }} \text { stage, } \mathrm{ML}=0.185
$$

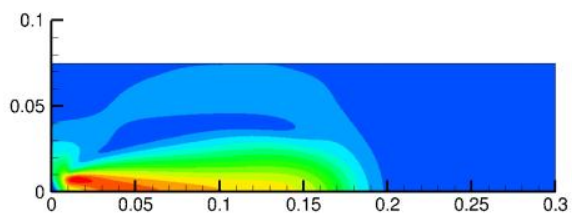

$220^{\circ}$
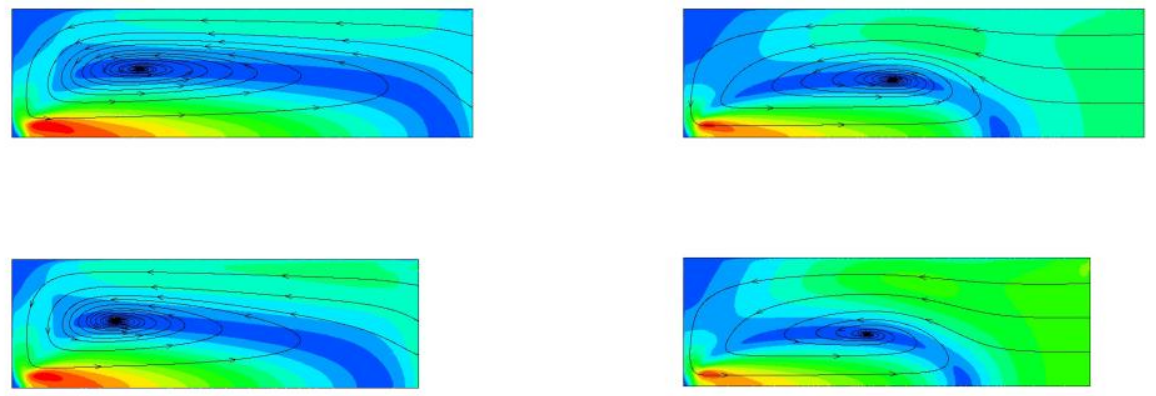

$270^{\circ}$
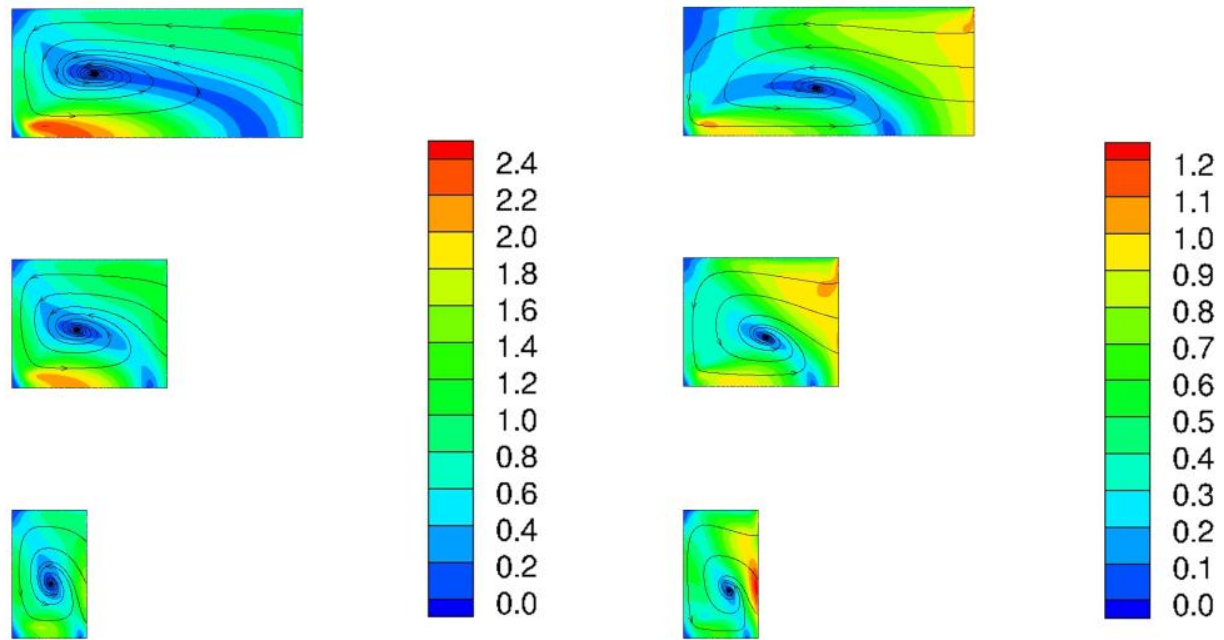

2.0

$300^{\circ}$

$(69 \%)$

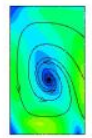

0.0

Fig. 6.3 Contours of gas velocity magnitude with streamlines [m/s] for equal total injected mass but different initial gas pressure

In Fig. 6.3, contours of gas velocity and streamlines are shown for the compression portions of the first-stage (Case 2) and the second-stage (Case 3). Before compression starts, gas is accelerated along the centerline by injected droplets. A large vortex is formed in the closed cylinder, which represent a toroidal vortex when 
considered in three-dimensions. In order to satisfy mass conservation, gas is moving and cycling in the chamber. In both cases, the same nozzle with same liquid flow rate is used. In the second stage, the gas velocity is smaller due to a denser gas.

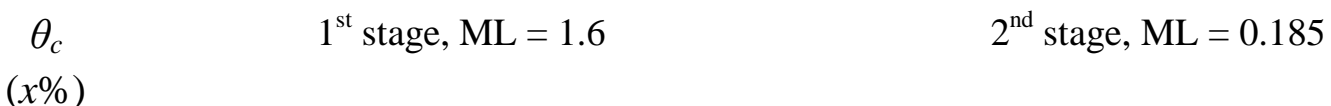

$180^{\circ}$

$(0 \%)$
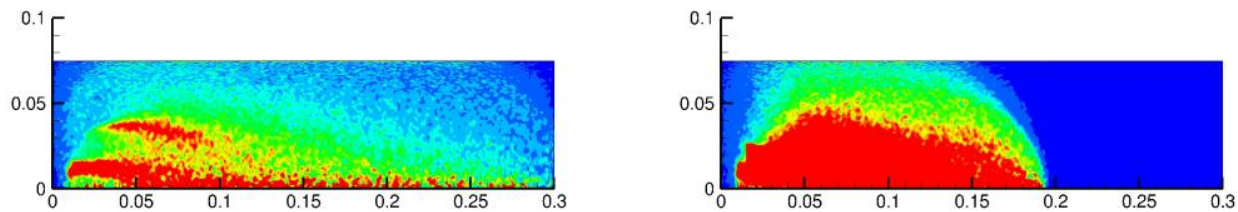

$220^{\circ}$

$(9 \%)$
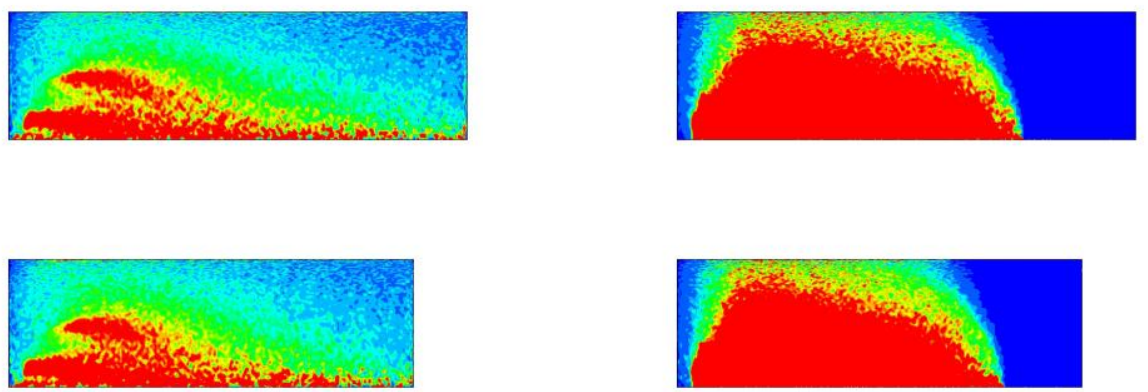

$270^{\circ}$
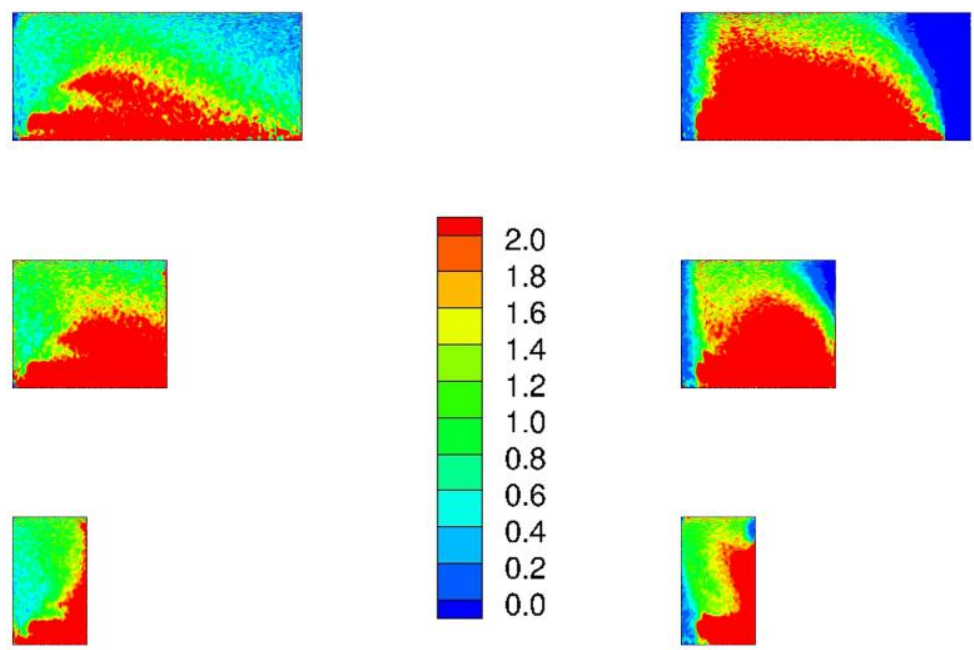

Fig. 6.4 Contours of droplet transient mass loading 


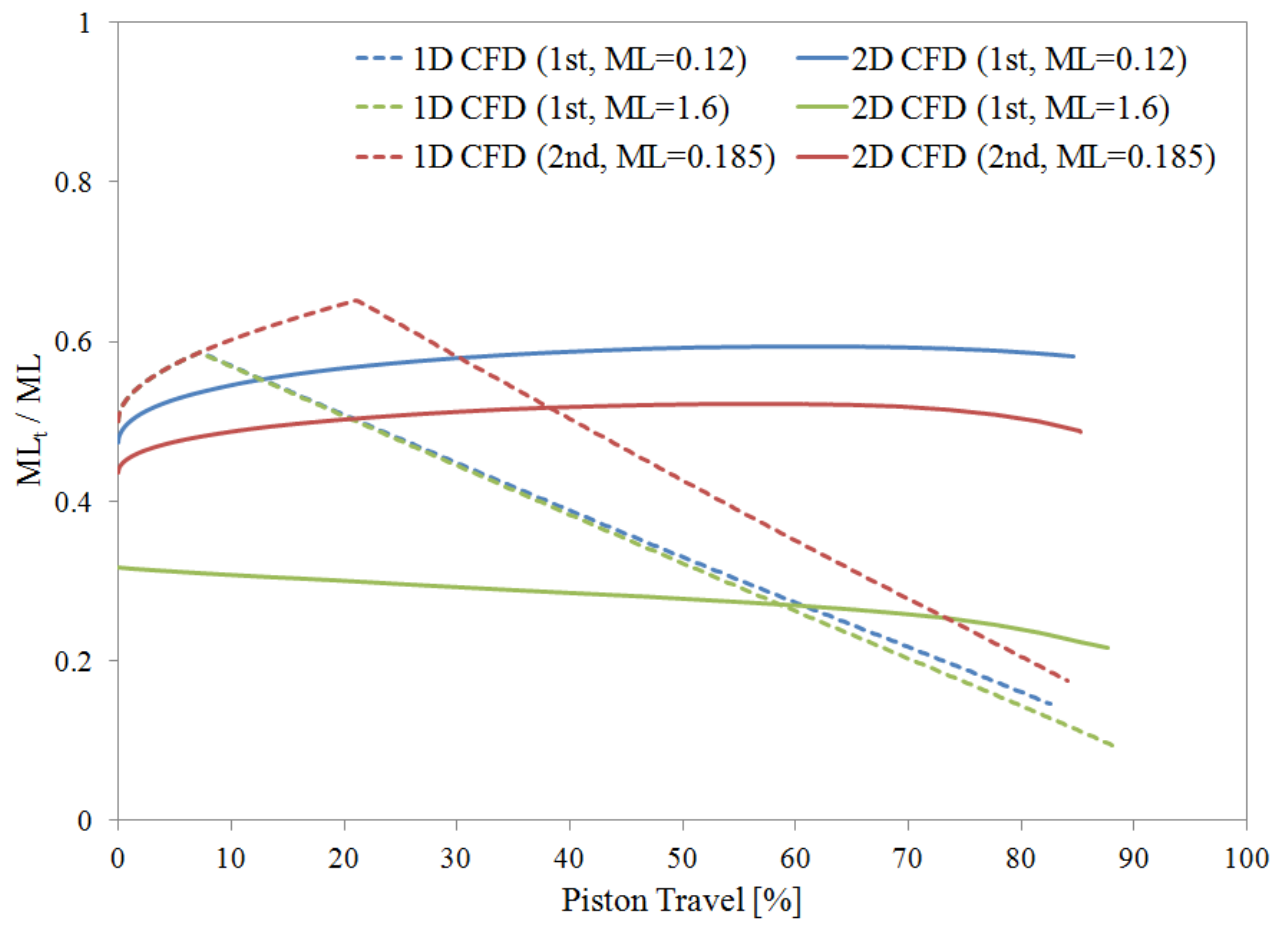

Fig. 6.5 Transient mass loading normalized by total injected mass loading as a function of the piston travel (normalized \% by chamber length) during compression (where $0 \%$ corresponds to a crank angle of $180^{\circ}$ )

The effect of initial pressure on the distribution of droplet mass loadings is shown in Fig. 6.4 for Cases 2 and 3 and it can be found that droplet distribution is not spatial uniform. In the first-stage, the droplets have propagated all the way to the piston wall by $\theta_{c}=180^{\circ}$, whereas the second stage, droplets decelerate very soon because in denser gas large drag forces act on droplets. Thus, droplets in the second stage cannot reach the piston surface which causes zero-mass-loading zone (blue colored) near the piston surface. Meanwhile, a uniform zone of relative high mass loading (red colored) occurs in the second stage due to the increased drag of the drops at higher gas density. On the contrary, droplets are dispersed well in both radial and axial directions in the first stage chamber relative mass loading to the injected mass loading is smaller due to droplet 
losses on the piston and sidewalls. To get both high mass loading and uniform distribution, one approach is to employ multiple nozzles mounted on the side walls. But this multi-nozzle configuration is no longer axisymmetric and hence requires a threedimensional simulation.

The total injected mass loading indicates how much liquid is injected into the compression cylinder during the whole cycle. However, due to droplet loss effects, the amount of droplets aloft in the chamber instantaneously will be less than the injected mass loading. Herein, the transient mass loading normalized by the total injected mass loading is plotted in Fig. 6.5 for all cases. Typically, there are three possible surfaces for droplet losses: the piston surface, the sidewall and the cylinder head. In the onedimensional (1D) simulations, only the first loss (piston surface) is modeled and since the droplet do not fall fast enough to hit the piston before $\theta_{c}=180^{\circ}$, there is no loss before this crank angle. Since half the spray has been discharged for this half-cycle time, the normalized mass loading at $\theta_{c}=180^{\circ}$ is 0.5 . As shown in Fig. 6.4, the $1 \mathrm{D}$ mass loadings continue to increase due to spray injection but as droplets begin to hit the piston surface, there is a discontinuous change in slope (e.g. at $8 \%$ piston travel for the first-stage systems) as the mass loading begins to decrease. The onset of this decrease is delayed in the second stage to about $22 \%$ since momentum of droplets is absorbed by the denser gas, so time of droplets take longer to reaching the piston surface. For the two-dimensional cases, large injected mass loading leads to droplet losses before the compression starts so that the transient mass loading is already below 0.5 by $\theta_{c}=180^{\circ}$. This is a result of the higher gas velocity along the centerline (caused by two-way coupling) as shown in Fig. 6.3 that causes the droplets to move faster in the axial direction thereby impacting the 
piston but also leads to sidewall impact due to finite radial velocities (which were neglected in the one-dimensional simulations). This effect is particularly significant for the $\mathrm{ML}=1.6$ case which is consistent with the high centerline gas velocities associated with this case. However, at longer times the 2D results show a reduced amount of droplet losses compared to the $1 \mathrm{D}$ cases. This can be attributed to the cylinder vortex which causes the droplets to recirculate within the chamber and stay aloft droplets longer. Thus, this vortex has the advantage of improving heat transfer by allowing a higher mass loading at later portions of the piston stroke.

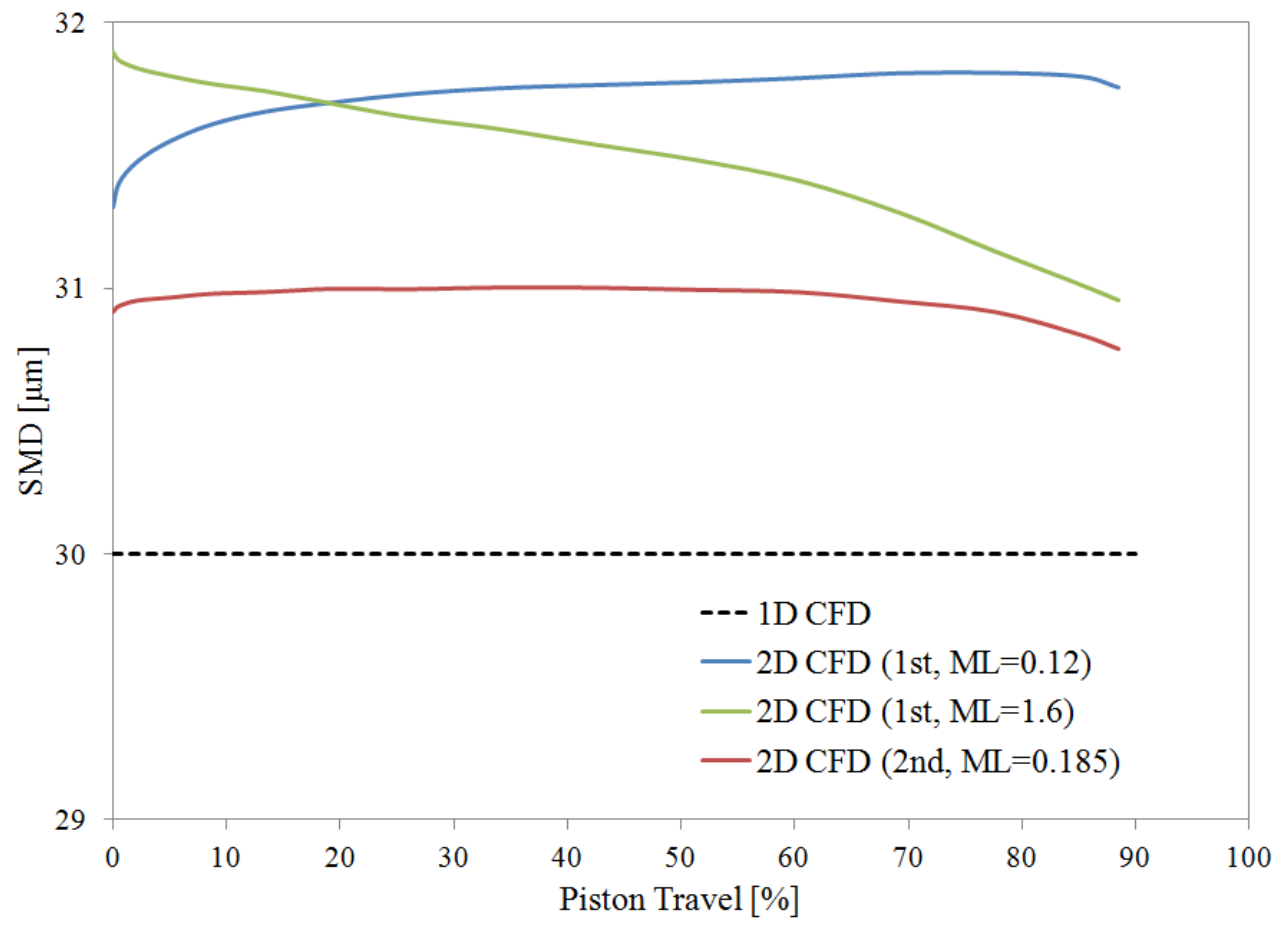

Fig. 6.6 Mean droplet size in the compression process

While small particles tend to follow the flow, the velocity of large particles does not undergo substantial changes because of their large momentum. In Fig. 6.6, droplet mean size is shown during the compression. If the mean size goes up, that means that 
small droplets are lost; conversely, if the mean size goes down, that means that large droplets are lost. For the case with $\mathrm{ML}=1.6$, large droplets move fast and they are lost by hitting walls. However, for the case with $\mathrm{ML}=0.12$, more small droplets are lost. Usually small droplets are decelerated quickly compared to large droplets, so small droplets cannot reach wall earlier than large droplets. The explanation of small droplet losses is that small droplets are affected by turbulent fluctuations and these small droplets laterally disperse and hit the sidewall. Thus, large droplet losses mainly are caused by their large momentum; small droplet losses mainly are caused by turbulent diffusion. In Fig. 6.4, the case with $\mathrm{ML}=1.6$ is dominated by the former, the case with $\mathrm{ML}=0.12$ is dominated by the latter, and the case with $\mathrm{ML}=0.185$ is resulted from both. Thus, the turbulent effect is more important to small droplets losses. However, the effects on droplet size are generally weak as the mean diameter change by less than $10 \%$ for all three cases.

Based on the mass loading and droplet size, the total inject area can be determined and is shown in Fig. 6.7. Despite a small mass of liquid injected ( 1 gram), droplets can provide a huge surface area $\left(\sim 1 \mathrm{~m}^{3}\right)$ for heat transfer! The results also indicate that the 1D model over-estimates the injected area at the initial times, because the key droplet loss mechanisms are not included. Interestingly, the net area is approximately the same as the $2 \mathrm{D}$ by the end of the compression cycle. Thus, the 1D underestimation of losses in the initial part of the cycle is balanced by an overestimation of losses in the latter part of the cycle. 


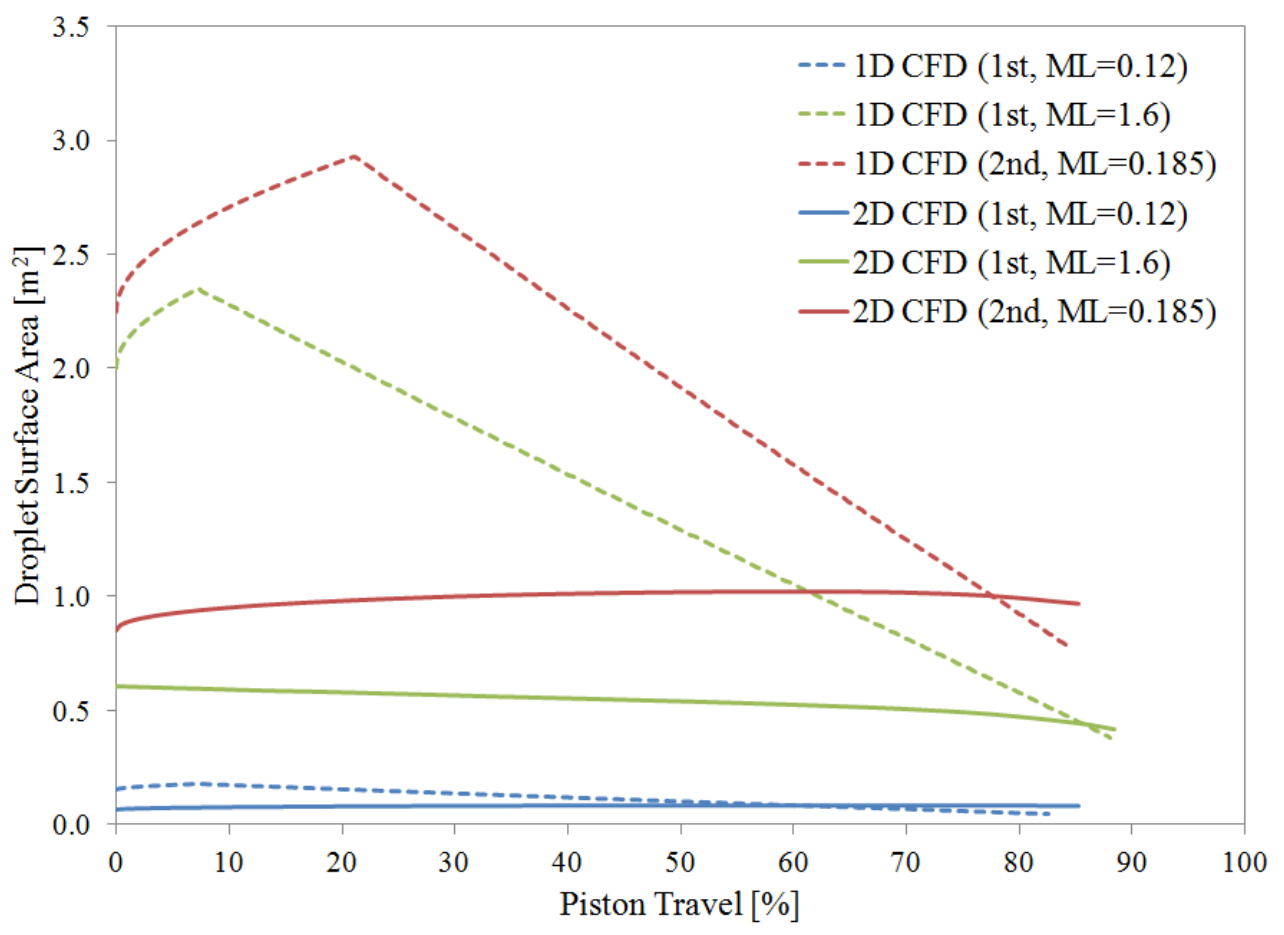

Fig. 6.7 Total droplet surface area aloft at a given time

In an ideal condition, droplets are injected uniformly during the compassion and heat transfer occurs consistently throughout the chamber space at the same rate. In this case, the gas temperature increase can be controlled uniformly and no temperature gradient appears. In reality, it is difficult to achieve a uniform gas temperature distribution as shown in Fig. 6.8. In particular, regions of high mass loading have high heat transfer and small temperature increases, stronger temperature gradients arise at the latter stages as the mass loading has reduced and is the droplet concentration is more skewed spatially. This is particularly evident in second stage compression where a large temperature differential of $100 \mathrm{~K}$ occurs due to small mass loading with uneven distribution. Thus, regions of small mass loading are important to avoid as high heat transfer is desired. 


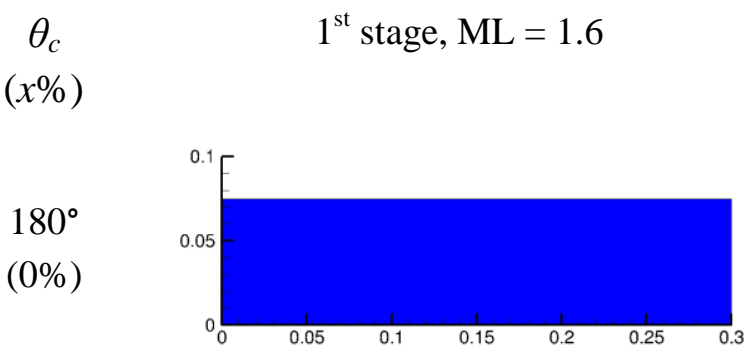

$$
2^{\text {nd }} \text { stage, } \mathrm{ML}=0.185
$$

$220^{\circ}$

(9\%)
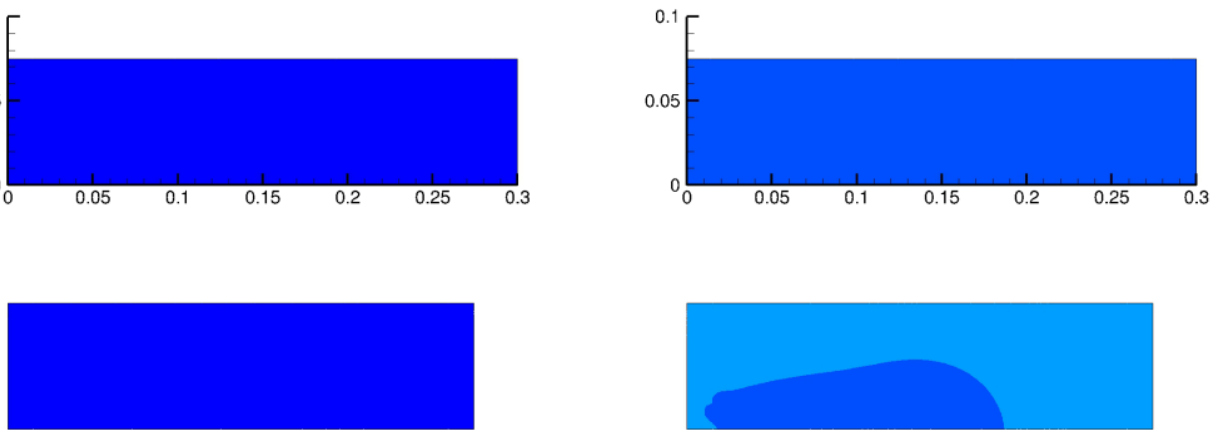

$240^{\circ}$

(19\%)
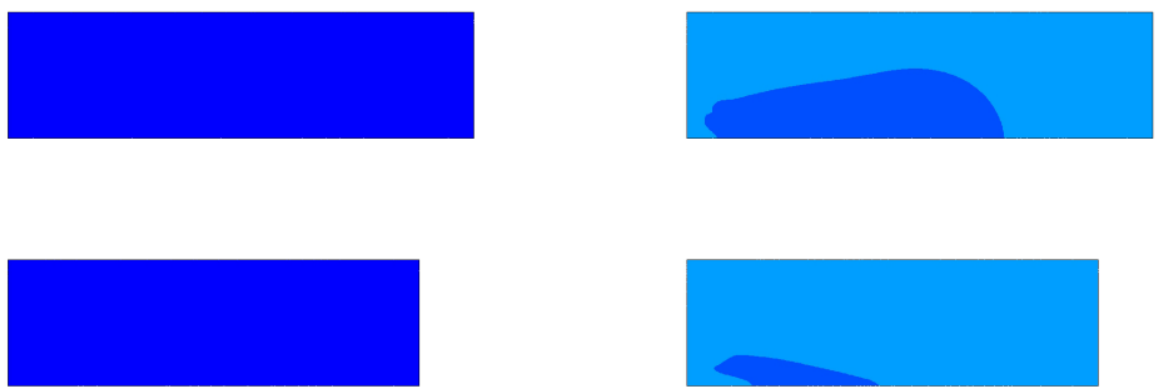

$270^{\circ}$

(42\%)
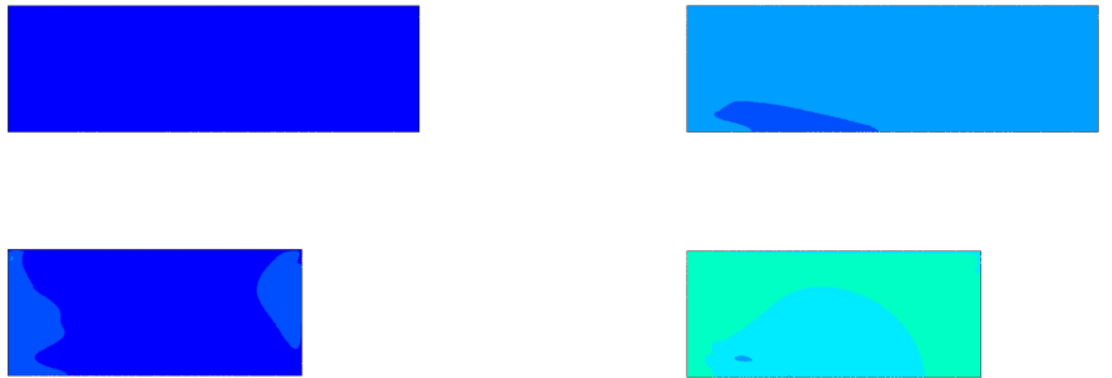

$300^{\circ}$

(69\%)
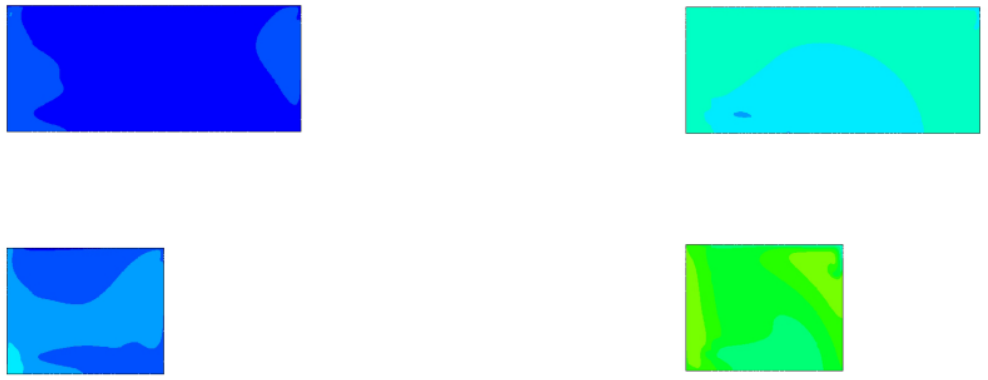

$320^{\circ}$

(85\%)
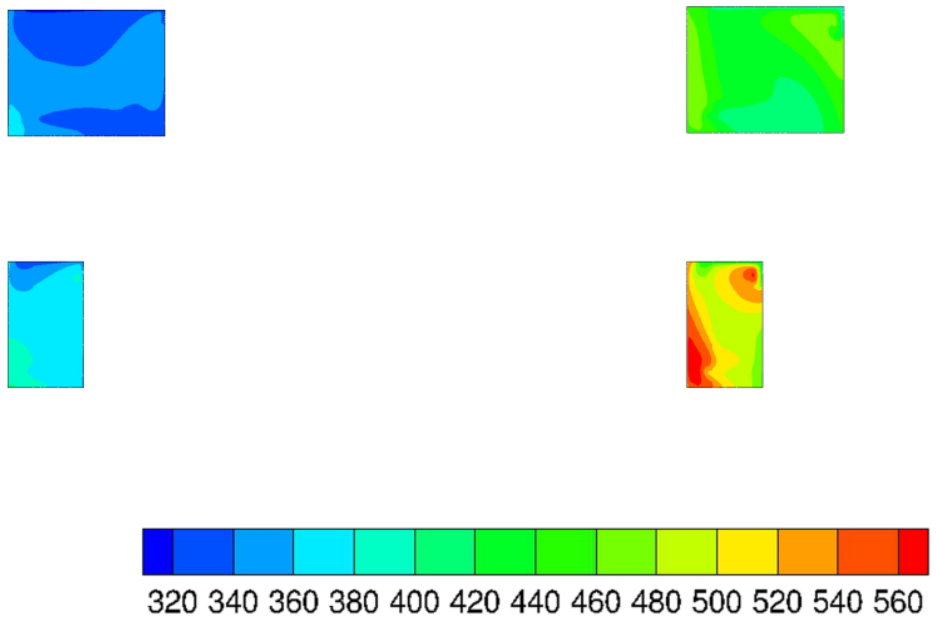

Fig. 6.8 Contours of gas temperature [K]

As a result of the droplet heat transfer, the mass-average air temperature is reasonably controlled in compression process as shown in Fig. 6.9. It is important to note 
that, when the pressure becomes getting close to the desired pressure, the droplet heat transfer is critical. This is because, at that time, a small piston travel causes a large increase in both pressure and temperature and large generated heat needs transfer to droplets. Recalling the results of Fig. 6.7, the 1D cases have large heat transfer area initially as compared to the $2 \mathrm{D}$ cases. Thus, to achieve effective droplet heat transfer, it is critical to having large heat transfer area when pressure and temperature increase rapidly.

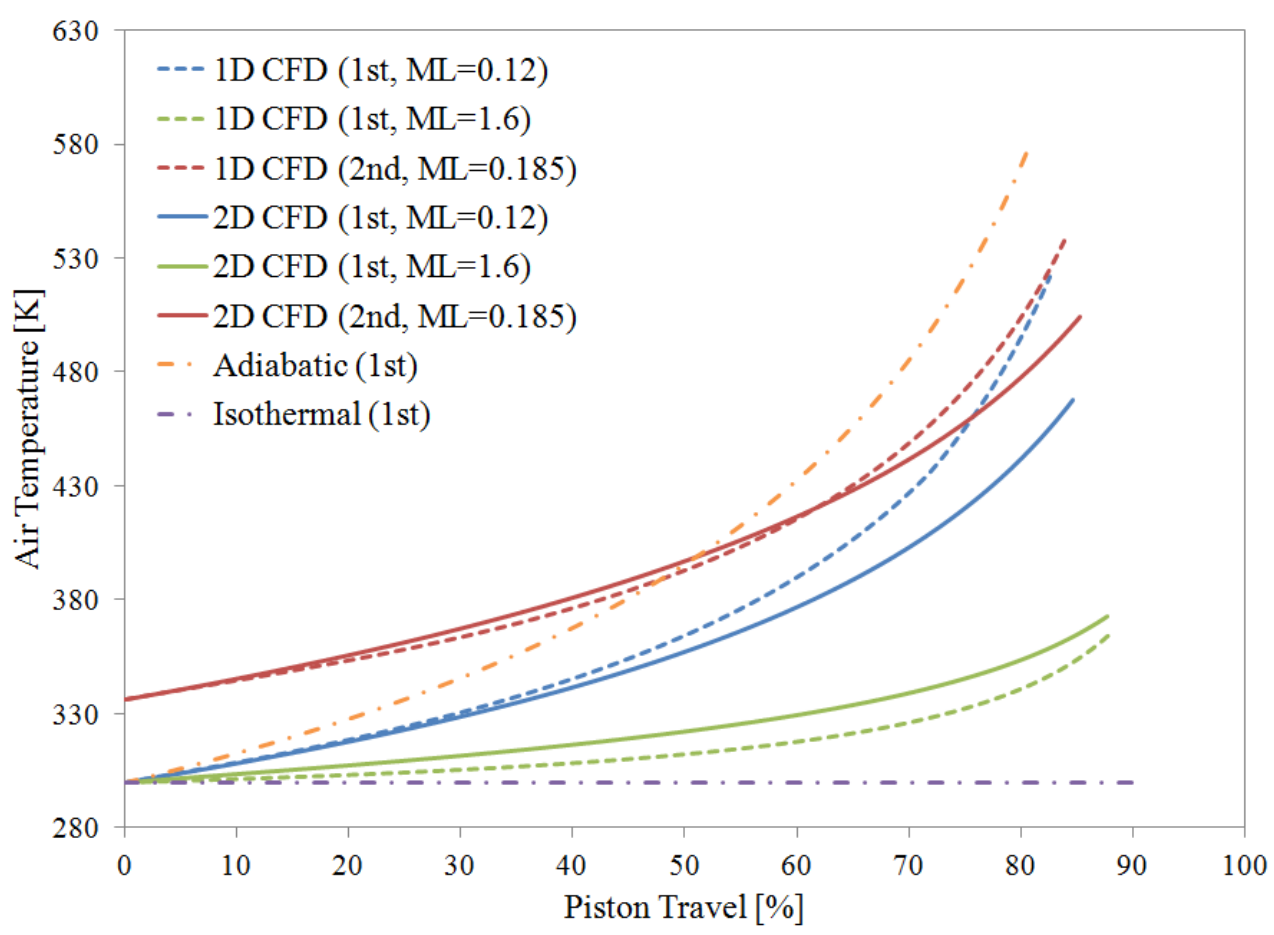

Fig. 6.9 Air temperature increase caused by compression

To describe compression thermodynamics, pressure-volume curves are shown in Fig. 6.10. In order to make the first and second stages comparable, a pressure ratio is used for the vertical axis. The results show that injected mass loading primarily determines the performance of compression for both $1 \mathrm{D}$ and $2 \mathrm{D}$ simulations. This is 
evidenced by comparing cases with similar mass loading but different spray conditions, whose p-v curves are close to each other. For large mass loading, both 1D and 2D models show a substantial improvement closer to isothermal conditions.

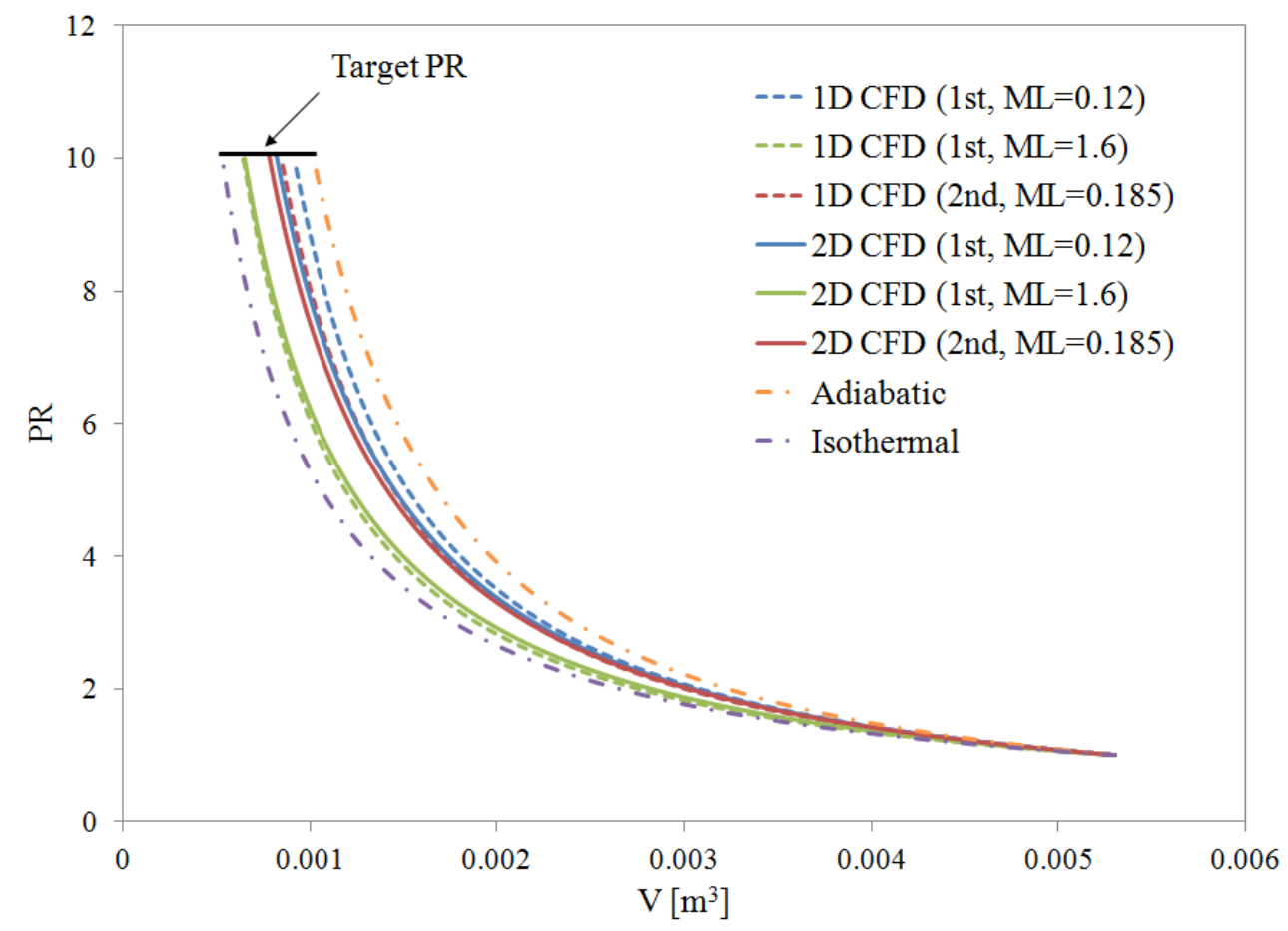

Fig. 6.10 Pressure ratio as a function of piston volume during compression

In Fig. 6.11, the above compression thermodynamics can be used to obtain the polytrophic index as a function of piston travel. Comparison with Fig. 6.5 indicates that this polytrophic index is strongly related to mass loading and droplet losses and that variation of this index is small especially for large mass loading cases. Integrating these curves over time, an average polytrophic index can be employed to determine the overall compression performance. 


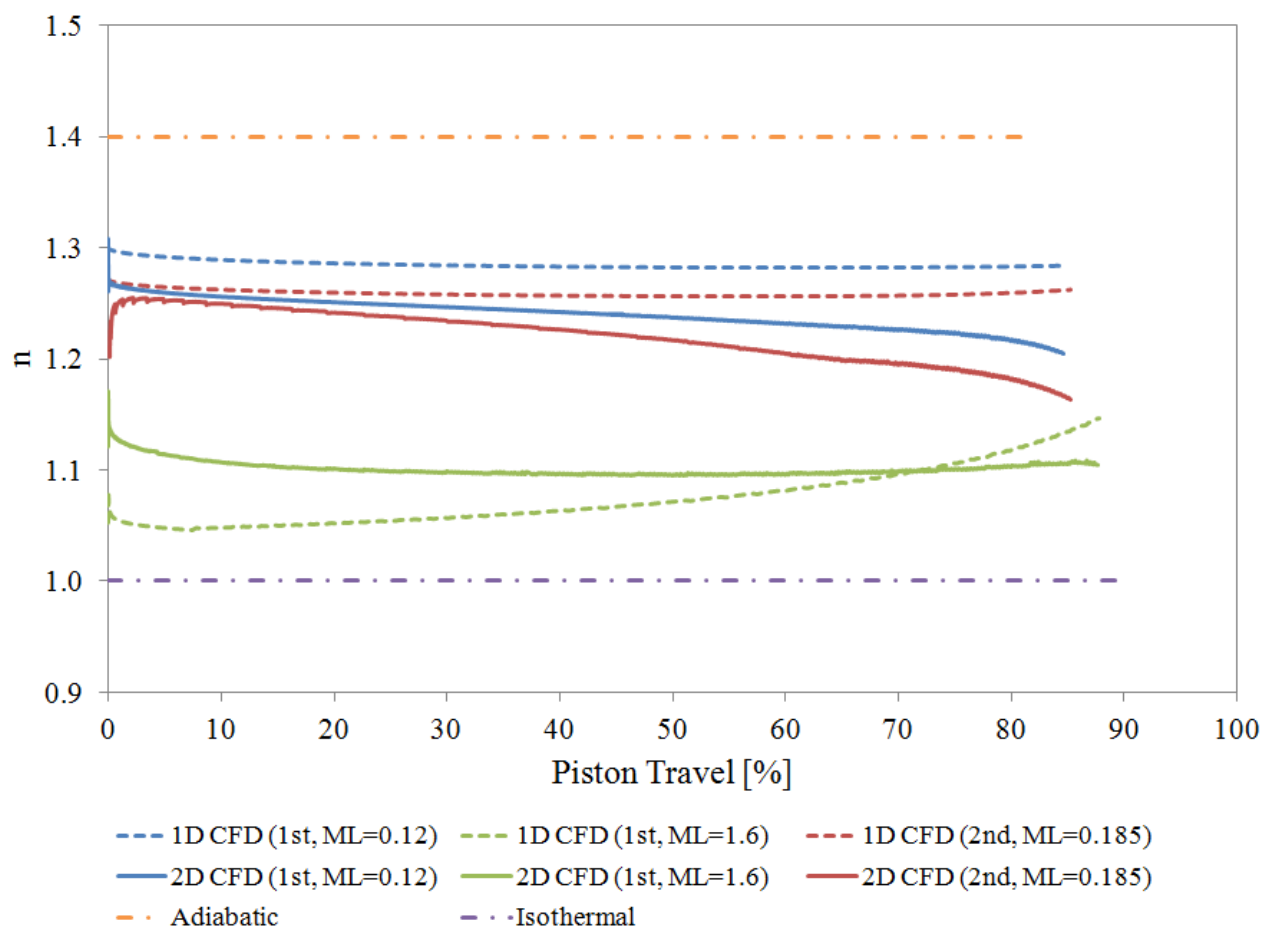

Fig. 6.11 Polytrophic index of compression process

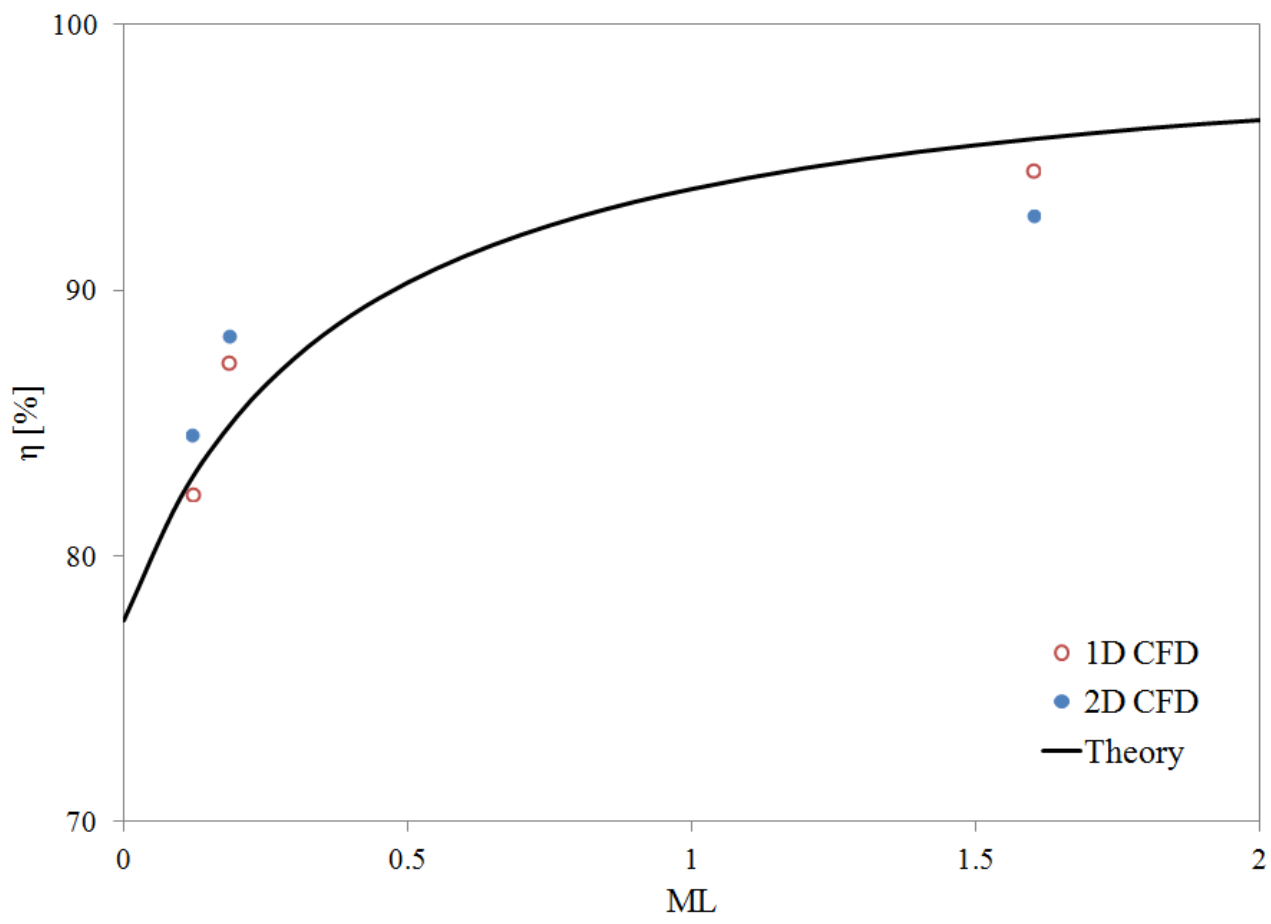

Fig. 6.12 Compression efficiency for different injected mass loading 
These overall compression efficiencies are estimated in Fig. 6.12 for all of the cases. With large injected mass loading, the efficiency is significantly higher than $90 \%$ for both $1 \mathrm{D}$ and $2 \mathrm{D}$ results, and not far away from the theoretical result based on total injected mass loading and droplet losses due only to droplets moving at terminal velocity when hitting the piston surface. In some cases, the simulations actually give higher efficiency than theory. The 2D cases with small mass loading indicate higher efficiency than theory because of the vortex formed in the chamber which tends to prevent droplet losses. Another exception is the 1D case of the second stage, where the condition is different to the condition used in theory. Despite these differences between 1D and 2D simulations as well theory, it is interesting to note that the compression efficiency can be reasonably estimated to be primarily a function of mass loading for present conditions. 


\section{Chapter 6 References}

[1] Greenblatt JB, Succar S, Denkenberger DC, Williams RH, Socolow RH. "Baseload Wind Energy: Modeling the Competition Between Gas Turbines and Compressed Air Energy Storage for Supplemental Generation". Energy Policy 2007; 35: 1474-1492.

[2] Li PY, Loth E, Simon TW, Van de Ven JD, Crane SE. "Compressed Air Energy Storage for Offshore Wind Turbines". 2011 International Fluid Power Exhibition (IFPE), Las Vegas, NV, March, 2011.

[3] Qin C, Loth E. "Liquid Piston Compression with Droplet Heat Transfer", Applied Energy 2014; 114: 539-550.

[4] Qin C, Loth E, Li P, Simon T, Van de Ven J, Crane SE, Pourmousa A. Spray-Cooling Concept for Wind-Based Compressed Air Energy Storage. 11th International Energy Conversation Engineering Conference (IECEC) 2013, San Jose, CA.

[5] Launder BE, Spalding DB, "Lectures in Mathematical Models of Turbulence", London, England: Academic Press; 1972.

[6] Loth E. "Quasi-steady shape and drag of deformable bubbles and drops", International Journal of Multiphase Flow 2008; 34(6): 523-546.

[7] Morsi SA, Alexander AJ. "An Investigation of Particle Trajectories in Two-Phase Flow Systems", Journal of Fluid Mechanics 1972; 55(2): 193-208.

[8] Bocksell TL, Loth E. "Random Walk Models for Particle Diffusion in Free-Shear Flows", AIAA Journal 2001; 39(6): 1086-1096.

[9] Hinze JO, Turbulence, McGraw-Hill, New York 1975.

[10] Wang XF, Lefebvre AH. "Mean Drop Sizes from Pressure-Swirl Nozzles", Journal of Propulsion 1987; 3(1): 11-18. 


\section{Chapter 7 Simulation of Spray in a Premixed Feeder Tube}

\subsection{Introduction}

Compressed air energy storage has become recently of interest for a number of applications including use in off-shore wind turbines ${ }^{[2]}$. To achieve high efficiency

needed for energy storage and recovery, Qin and Loth ${ }^{[3]}$ showed that a large number of small droplets directly injected into the chamber can help achieve a nearly isothermal process during compression. However another method is to inject droplets by pre-mixed injection, for example as demonstrated in Fig. 7.1. For this pre-mixed injection, water spray is injected in a feeder tube and mixed with inlet gas upstream. Then the mixture of droplets and gas goes though the intake valve to fill the entire chamber with droplets at the start of the compression process. In addition, a switch valve can be employed to allow a feeder tube to feed a couple of compression cylinders. This valve allows compression of air in piston \#2 as it undergoes an upward piston stroke while the feeder tube is used to supply drops and air to piston \#1 as it undergoes a downward piston stroke. As the two pistons switch the direction of motion at a later time, so does the switch valve.

In the present paper, multi-phase computational fluid dynamic (CFD) simulations are implemented in a domain of a feeder tube (shown as a dashed box in Fig 7.1). This simulation is helpful to understand impact of a feeder tube and its geometry, in order to achieve a uniform spatial droplet feed inside a compression chamber. Based on authors' knowledge, this study is the first to simulate the axisymmetric mixture process between the gas and spray in a feeder tube. It is also the first to examine the impact of feeder tube 
diameter and length with respect cross-sectional performance as measured the degree of radial uniformity of droplet mass flux and the degree of droplet mass loss due to collisions with sidewalls. Understanding the impact of such performance can help to determine future designs of both the feeder tube and intake valve coupled with piston geometries.

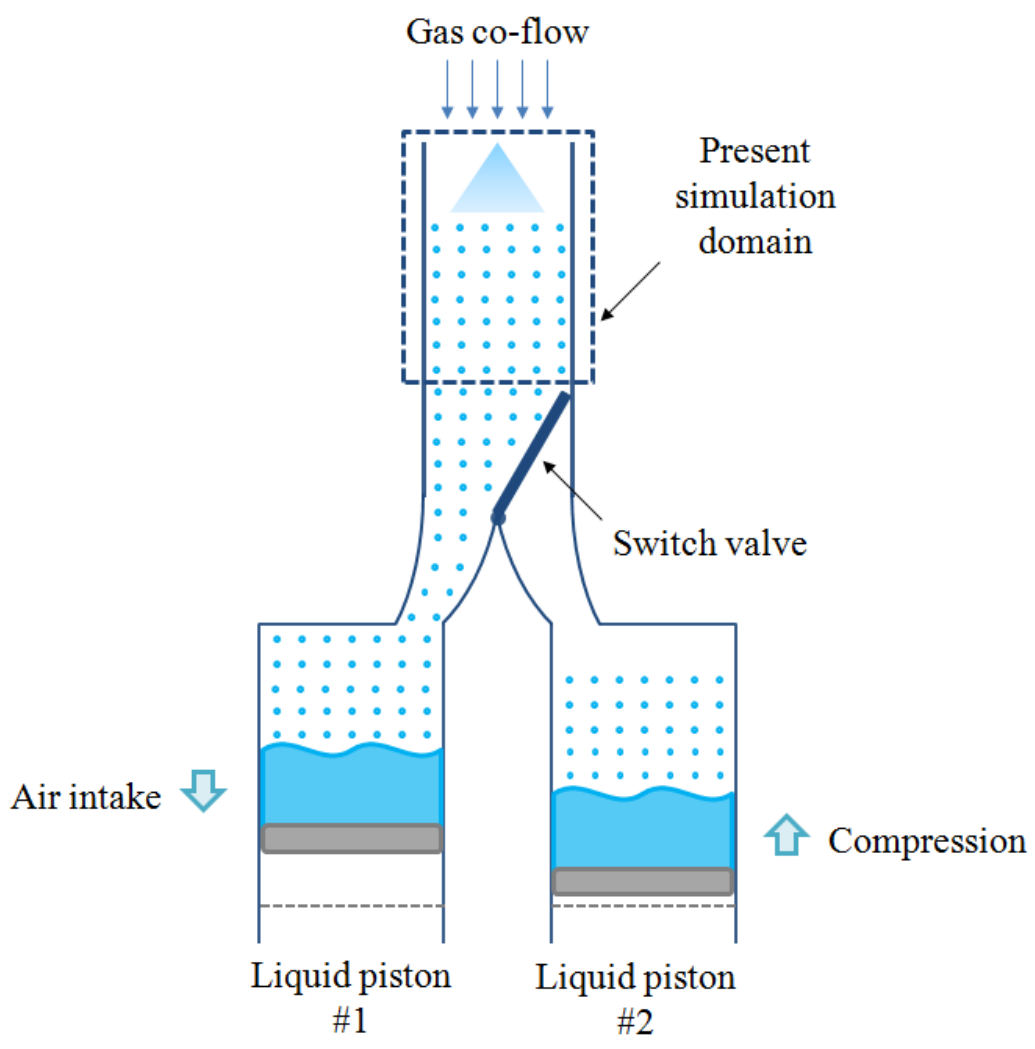

Fig. 7.1 Sketch of pre-mixed injection

\subsection{Methodology}

\subsubsection{Numerical Methods}

In the present chapter, an Eulerian numerical approach is chosen for the gas phase and the Lagrangian approach for the liquid phase. To ensure both numerical stability and 
low computational cost, the continuous phase (ambient gas) is solved by Reynoldsaveraged Navier-Stokes (RANS) equations, using the standard k- $\varepsilon$ model, while the dispersed phase (droplet) is solved by trajectory discretization with a discontinuous random walk (DRW) model to produce synthetic turbulent fluctuations.

The gas-phase is treated with a standard k- $\varepsilon$ model ${ }^{[5]}$ whereby the turbulent kinetic energy $(k)$ and its dissipation rate $(\varepsilon)$ are solved in two transport equations:

$$
\begin{aligned}
& \frac{\partial}{\partial t}\left(\rho_{g} k\right)+\frac{\partial}{\partial x_{i}}\left(\rho_{g} k u_{i}\right)=\frac{\partial}{\partial x_{j}}\left[\left(\mu_{g}+\frac{\mu_{t}}{\sigma_{k}}\right) \frac{\partial k}{\partial x_{j}}\right]+G_{k}+G_{b}-\rho_{g} \varepsilon-Y_{M} \\
& \frac{\partial}{\partial t}\left(\rho_{g} \varepsilon\right)+\frac{\partial}{\partial x_{i}}\left(\rho_{g} \varepsilon u_{i}\right)=\frac{\partial}{\partial x_{j}}\left[\left(\mu_{g}+\frac{\mu_{t}}{\sigma_{k}}\right) \frac{\partial \varepsilon}{\partial x_{j}}\right]+C_{1 \varepsilon} \frac{\varepsilon}{k}\left(G_{k}+C_{3 \varepsilon} G_{b}\right)-C_{2 \varepsilon} \rho_{g} \frac{\varepsilon^{2}}{k}
\end{aligned}
$$

In equation (7-1) and (7-2), $u_{i}$ refers to gas velocity in tensor notation, $x_{i}$ refers to the Eulerian coordinates in tensor notation, $\rho$ is density, and the subscript $g$ indicates a gas-phase property. Herein, the constants and non-dimensional numbers have the following values: $C_{1 \varepsilon}=1.44, C_{2 \varepsilon}=1.92, C_{\mu}=0.09, \sigma_{k}=1.0, \sigma_{\varepsilon}=1.3$. In the above expressions, turbulent viscosity $\left(\mu_{t}\right)$ is calculated by:

$$
\mu_{t}=C_{\mu} \rho_{g} \frac{k^{2}}{\varepsilon}
$$

The turbulent kinetic energy generation and distraction terms $\left(G_{k}, G_{b}\right.$, and $\left.Y_{M}\right)$ are described in Ref. 5. In this model, turbulent two-way coupling is not considered so that none of these terms are related to the presence of the droplets. Instead the two-way coupling between continuous phase and discrete phase is included through interphase 
interaction forces as a sink term for the mean gas momentum and interphase heat transfer as a sink term for the mean gas temperature.

To describe the trajectory of a discrete phase particle (droplet), the equation of motion for droplets with centroid velocity $v_{i}$ is expressed in a Lagrangian reference frame [6]:

$$
\frac{d v_{i}}{d t}=F_{D}\left(u_{i}-v_{i}\right)+\frac{\left(\rho_{d}-\rho_{g}\right)}{\rho_{d}} g_{i}
$$

In this expression, subscript $d$ indicates a droplet property and the forces applied to the droplet are assumed to be dominated by drag and gravity forces (which is reasonable given the high ratio of droplet density to gas density. The first term on the right hand side is drag force per particle mass per relative velocity and $F_{D}$ is defined as

$$
F_{D}=\frac{3 \mu_{g} C_{D} \operatorname{Re}}{4 \rho_{d} d^{2}}
$$

In this expression, $\mathrm{Re}$ is the drop aerodynamic Reynolds number defined along with the magnitude of relative velocity $(w)$ and the component of relative velocity in the $i$ direction $\left(w_{i}\right)$ as.

$$
\begin{aligned}
& \operatorname{Re} \equiv \frac{\rho_{g} d w}{\mu_{g}} \\
& w=\sqrt{w_{i} w_{i}} \\
& w_{i}=v_{i}-u_{i}
\end{aligned}
$$


A spherical droplet assumption is reasonable for Weber number less than unity ${ }^{[6]}$, and this criterion is appropriate for the droplets considered herein based on their size and relative. Thus, the drag coefficient $C_{D}$ for spheres is used and taken from Morsi and Alexander ${ }^{[7]}$.

The gas velocity in Eqs. 7-4 and 7-6 (needed for droplet instantaneous trajectories) can be expressed in terms of mean and fluctuation gas velocity values:

$$
u_{i}=\overline{u_{i}}+u_{i}^{\prime}
$$

The mean value (first term on RHS) is obtained from RANS solution. The fluctuation velocity is also important to include to properly capturing turbulent diffusion. To obtain this term synthetically from the gas turbulent kinetic energy and dissipation, the discrete random walk (DRW) model of Ref. 8 is employed. The DRW model is achieved with a random number $(\sigma)$, and an average fluctuation strength $\left(u_{r m s}^{\prime}\right)$. In particular, an instantaneous gas velocity fluctuation is held constant over an interaction time and based on random sampling for each directional component such that the net variation over many samples is consistent with the gas turbulent kinetic energy

$$
\begin{aligned}
& u_{i}^{\prime}=u_{r m s}^{\prime} \sigma_{i} \\
& u_{r m s}^{\prime}=\left(\frac{2}{3} k\right)^{1 / 2}
\end{aligned}
$$

In this expression, $\sigma$ represents three independent Gaussian random numbers whose mean value is zero but whose rms value is unity. A new random number is generated along the droplet path when the integration time along the path exceeds the 
droplet-eddy interaction time $\left(\tau_{\text {int }}\right)$. This interaction time is based on the minimum of the overall eddy lifetime $\left(\tau_{\Lambda}\right)$ and the eddy traversal time $\left(\tau_{\text {tra }}\right)$ which are defined below as

$$
\begin{gathered}
\tau_{\Lambda}=C_{\tau} \frac{k}{\varepsilon} \\
\tau_{t r a, i}=\frac{\Lambda_{i}}{w}
\end{gathered}
$$

In these expressions, $C_{\tau}$ is the eddy timescale coefficient (whose calibrated value is 0.21 ) and $\Lambda$ is turbulence integral length scale (which is related to $k$ and $\varepsilon)^{[8]}$.

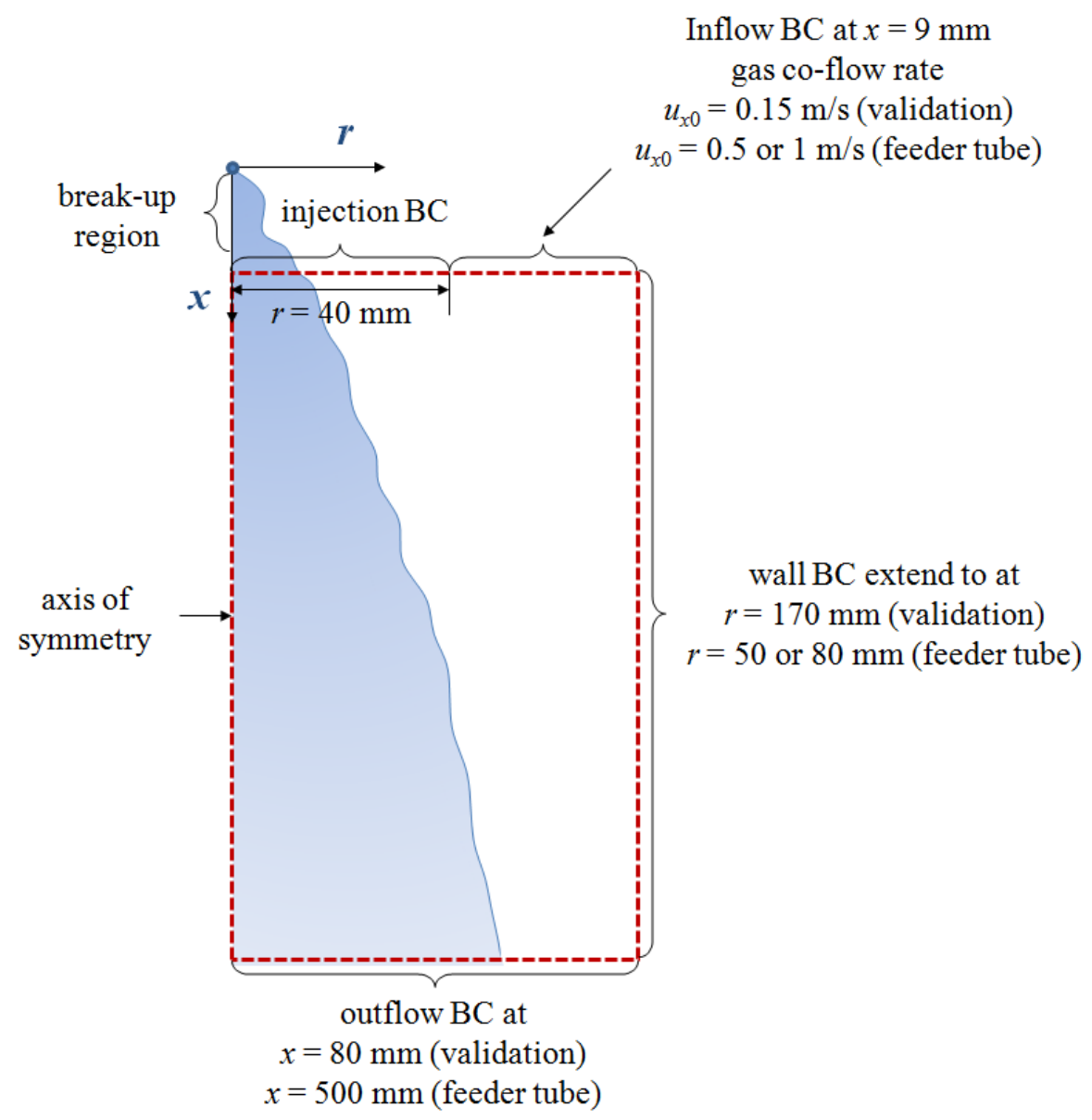

Fig. 7.2 Axisymmetric computational domain shown by red dashed box with inflow, wall and outflow boundary conditions for both validation case (based on Santolaya et al. geometry) and feeder tube cases 


\subsubsection{Validations with Santolaya et al. Data}

This present Eulerian-Lagrangian approach has been validated with experiments with similar test conditions as experienced by the feeder tube by examining comparisons of predicted and measured mean axial velocity of both gas and droplets and droplet radial mass flux distribution. The results indicated good agreement in Chapter 4 but it is also important to accurately predict the droplet radial velocities as well, because this the main characteristic that controls droplet wall loss in the feeder tube case. Thereof, the present study sought to evaluate the fidelity of the above approach with respect to droplet radial velocities for different size droplets. The computational domain for this validation case is based on the specific test condition in experiments done by Santolaya et al. ${ }^{[10]}$ as shown in Fig. 7.2, The domain is based on a cylindrical coordinate system referenced to the nozzle orifice as the origin for radial distance $(r)$ for axial distance $(x)$. Inflow and outflow boundary conditions are set at $x=9 \mathrm{~mm}$ and $x=80 \mathrm{~mm}$ respectively, while a wall boundary is set at $r=170 \mathrm{~mm}$. The gas mean velocity profile for the inflow boundary condition is described in Ref. 9. For the disperse phase, three bin sizes (small, medium, and large) spherical droplets are chosen to represent the polydisperse distribution. For each bin size, a single diameter is used: small at $7.5 \mu \mathrm{m}$, medium at 25 $\mu \mathrm{m}$, and large at $55 \mu \mathrm{m}$. The relative volumetric flow rates for the three sizes are: $2 \%$ for the small drops, $57 \%$ for the medium drops and $41 \%$ for the large drops. For the simulation, a uniform grid resolution of $\Delta x=\Delta r=1 \mathrm{~mm}$ was used along with a time step of $1 \mathrm{~ms}$. The values were found to be small enough to ensure grid and temporal independence while allowing reasonably computational efficiency. In each time step releases 2,410 particle parcels distributed radially over the injection plane at $x=9 \mathrm{~mm}$. 
Each parcel contains a sufficient number of particles to ensure that the injected droplet mass flux is maintained along with the proper radial distribution as described in previous chapters. The total number of parcels injected over a time of 5 seconds was found to be large enough to provide statistically convergent results.

Figure 7.3 shows the comparison between CFD (computational fluid dynamics) and experiment for different axial positions. At $x=18 \mathrm{~mm}$, the predicted both gas and small droplet radial velocities are relatively small, while the strong outward velocities are noted for the large droplets. The gas-phase and large droplet results are in reasonable agreement with experiment but the measured small and medium size droplets have a higher large radial velocity at outer radial positions, a region where few droplets are predicted to occur by the CFD. It is expected that these droplets are from break-up and collision of large droplets, because these droplets have similar velocity to large droplets in this same areas. It should be noted that droplet break-up and collision is not modeled in the CFD and this may be a significant effect for the small droplets. However, these droplets only represent $2 \%$ of the total mass flux and so this difference is not deemed critical.

\subsection{Feeder Tube Test Conditions}

The geometry of the computational domain for the feeder tube is based on this nozzle domain for the above validation (with an inflow at $9 \mathrm{~mm}$ downstream of nozzle orifice) and also based on the design described in Fig. 7.2. The feeder tube length was set at $500 \mathrm{~mm}$, but two different feeder tube radii are considered: $5 \mathrm{~cm}$ and $8 \mathrm{~cm}$. For droplet injection, a single concentrically-located pressure-swirl spray nozzle is employed. However, a higher droplet mass flux was employed than in the validation case in order to 
achieve a mass flux consistent with. This flux can be quantified in terms of mass loading (ML) defined at a given axial position of a feeder tube as:

$$
M L=\frac{\dot{m}_{d, i n j}}{\dot{m}_{g}}
$$

In this expression, $\dot{m}_{d}$ is the mass flux of droplet per unit time and unit crosssectional area and the subscript of inj indicates injection property. For the feeder tube condition, the maximum liquid mass flow rate experimentally obtained for a pressureswirl nozzle ${ }^{[11]}$ was used to provide the most realistic physically realizable condition for a compressed air energy storage system For compressed air energy storage, a mass loading of unity can provide compression efficiencies of about $87 \%$ for a pressure ratio of $10^{[3]}$. Based on a ML=1 and the gas flow velocity profile in the inner region $(r=40 \mathrm{~mm})$, the feeder tube gas co-flow rate at the outer edges was set as $0.5 \mathrm{~m} / \mathrm{s}$ for the $5 \mathrm{~cm}$ radius tube and at $1.0 \mathrm{~m} / \mathrm{s}$ for the $8 \mathrm{~cm}$ radius tube. 

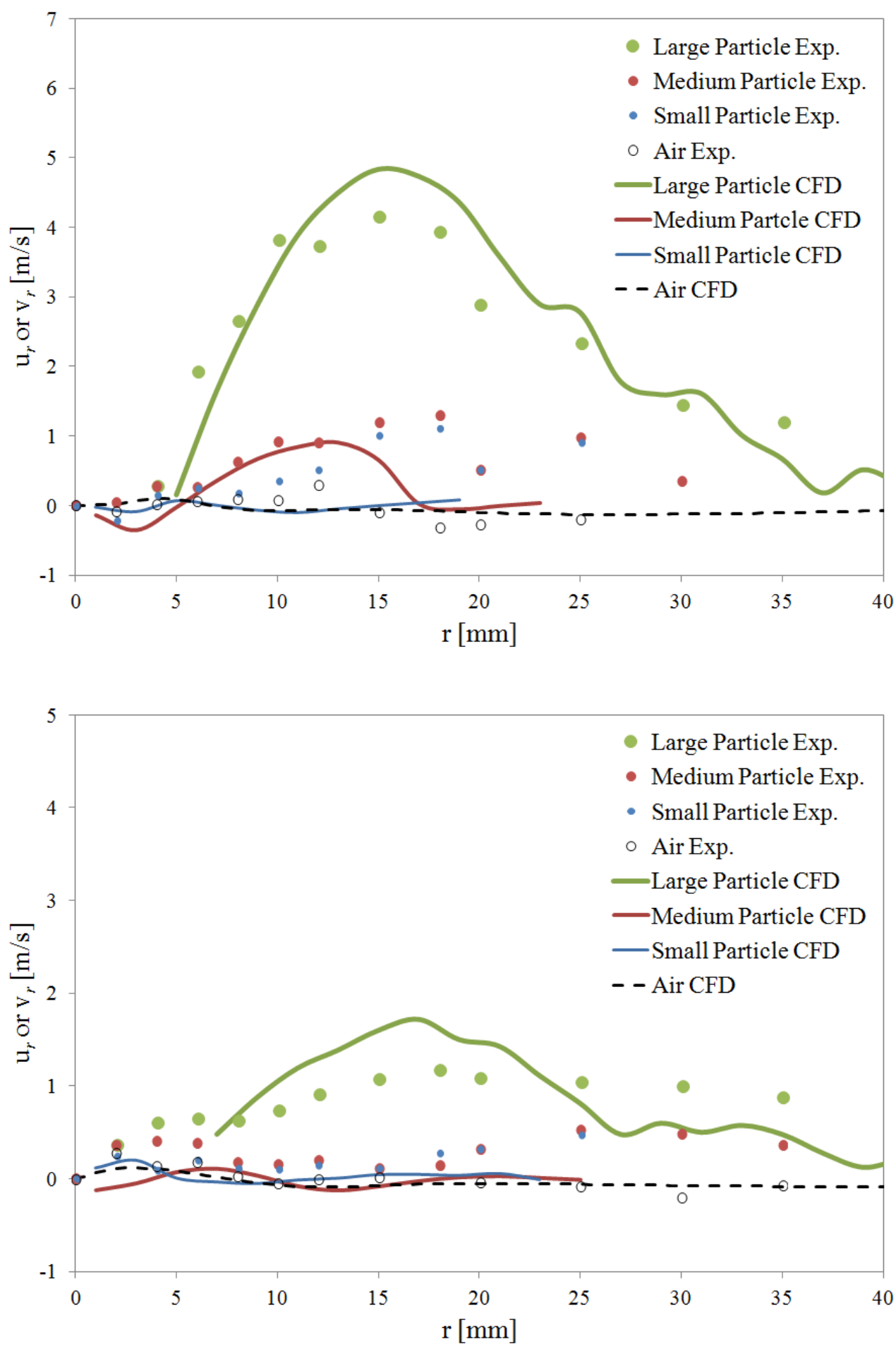

Fig. 7.3 Mean radial velocity comparison between CFD and experiment: a) at $x=18 \mathrm{~mm}$, b) at $x=36 \mathrm{~mm}$ 


\subsection{Results and Discussions}

In Fig. 7.4, mean gas velocity is shown in a 5-cm feeder tube. It can be seen that the flow field is similar to a jet flow with a high central gas velocity. When the axial distance increases, the central gas velocity decreases and a fully-developed velocity profile is reached. Thus, the length of the feeder tube cannot be short, when a flat gas velocity profile is necessary.
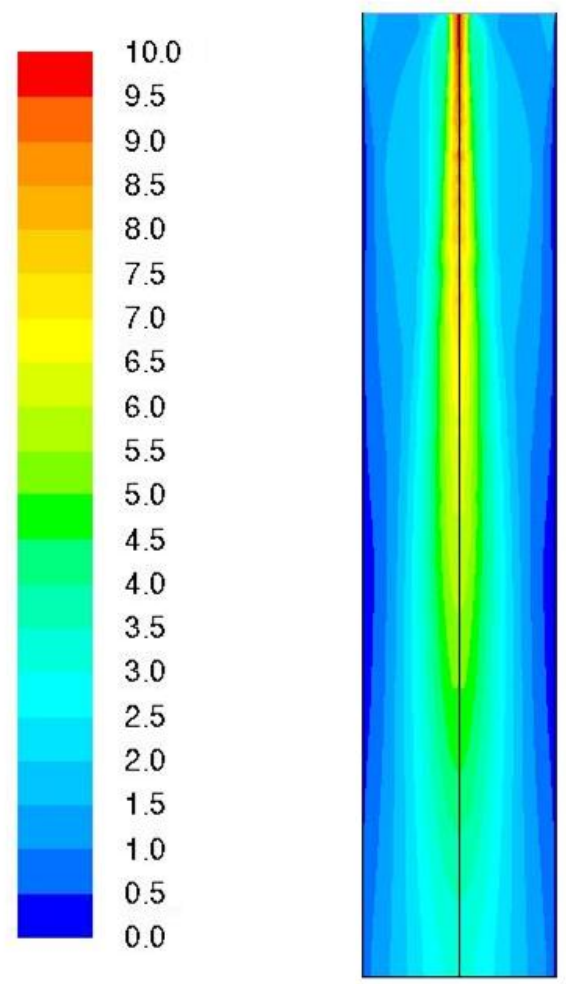

Fig. 7.4 Contours of mean gas velocity magnitude $[\mathrm{m} / \mathrm{s}]$

To demonstrate gas fluctuating velocity, turbulent kinetic energy is shown in Fig. 7.5 with a $5-\mathrm{cm}$ feeder tube. At beginning, the perturbation only happens in the center of the feeder tube. When the axial distance increase, due to coupling between gas and droplet phases, the perturbation spreads widely. If the axial distance goes further, 
turbulence begins to decay. As known, turbulence can help droplets disperse in radial direction, but lateral dispersion also causes droplet loss. Thus, in a feed tube, it is necessary to have a zone of uniform turbulence distribution to help droplets mix with gas, but this zone cannot to be too large, in order to avoid droplet loss.
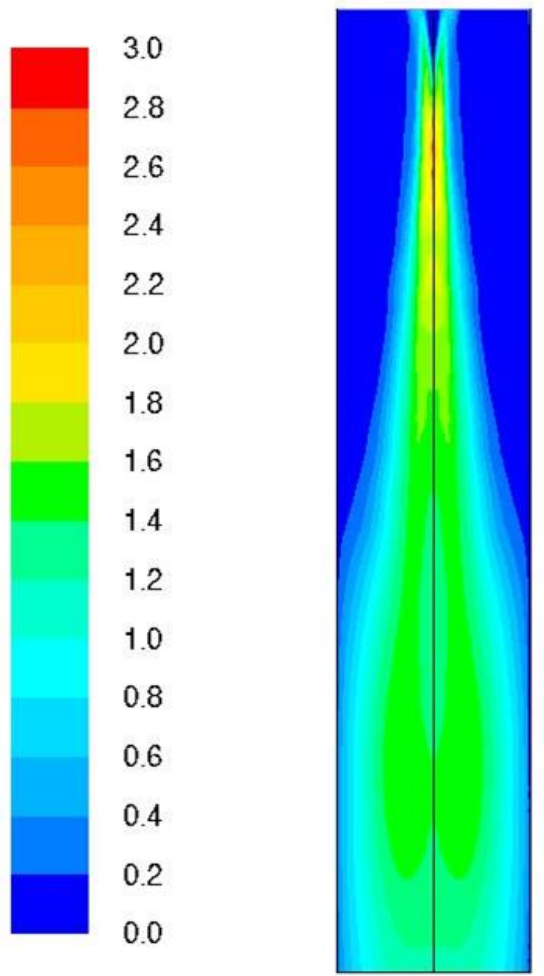

Fig. 7.5 Contours of gas turbulent kinetic energy $\left[\mathrm{m}^{2} / \mathrm{s}^{2}\right]$ 


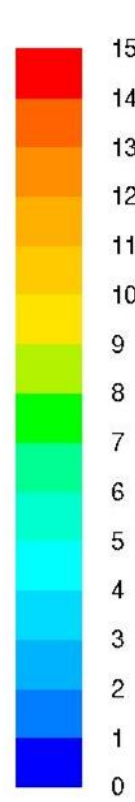

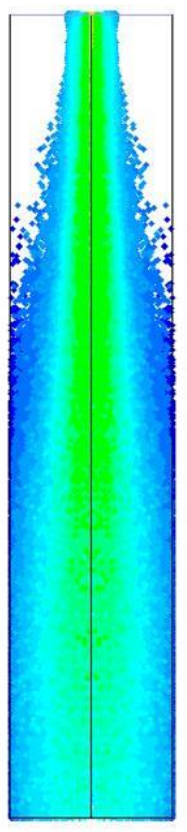

Small

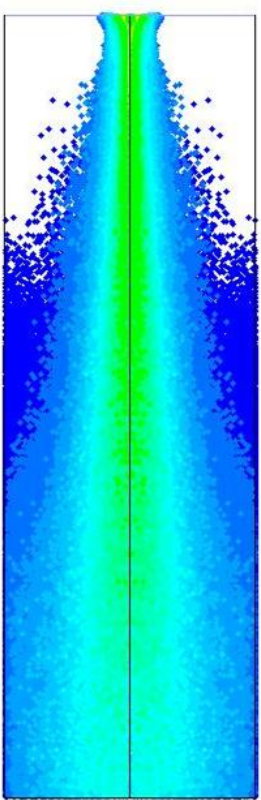

Medium

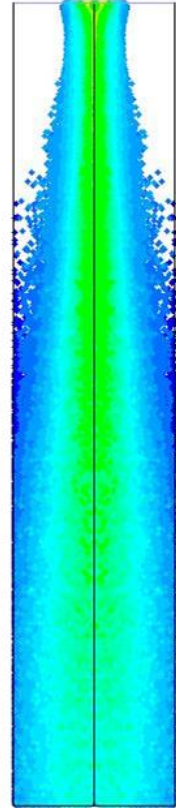

Medium

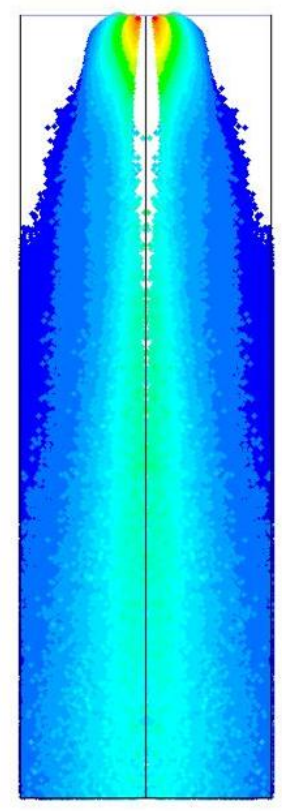

Large

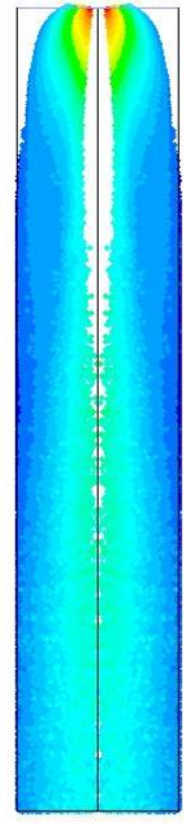

Large

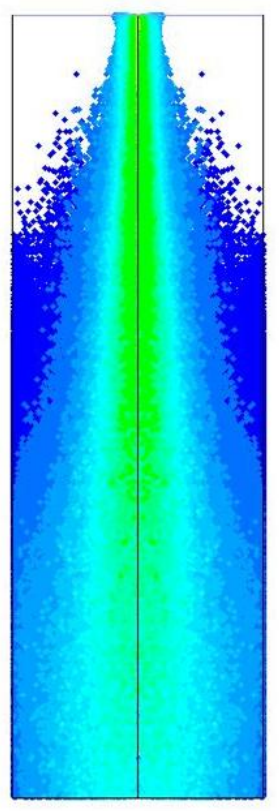

Small

Fig. 7.6 Particle positions colored by velocity magnitude $[\mathrm{m} / \mathrm{s}]$ : a) $5-\mathrm{cm} \mathrm{b)} 8-\mathrm{cm}$ diameter

Figure 7.6 shows droplet positions and mean velocities for different droplet sizes in both feeder tube geometries. First, it can be seen that small and medium droplets are 
similar to each other. When the axial distance increases, droplets spread in radial direction. Secondly, because large droplets have large momentum, droplet deceleration takes longer time compared to small and medium droplets. Also, it is worth noting that large droplets hit the side wall earlier than other sized droplets, and this droplet loss mainly caused by large radial velocities. In the third place, it can be found that the $5-\mathrm{cm}$ case is just like a cropped version of the 8 -cm case. In the 8 -cm case, near the side wall many small velocity droplets (colored in dark blue) are there. But in 5-cm case, these droplets are lost by hitting the wall. This also indicates, changing the diameter from $8 \mathrm{~cm}$ to $5 \mathrm{~cm}$, the effect of side walls on inner droplets is limited.

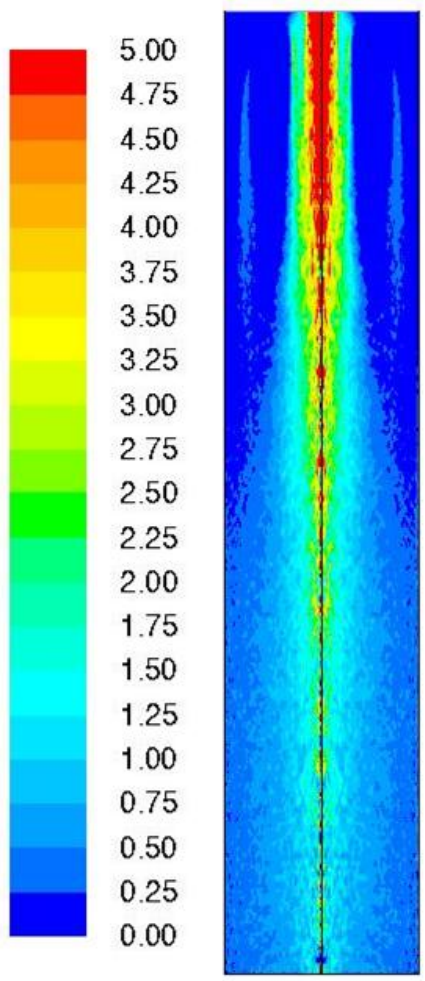

Fig. 7.7 Contours of particle concentration $\left[\mathrm{kg} / \mathrm{m}^{3}\right]$ 
In order to check the spatial mass distribution of droplet, droplet mass concentration, which is droplet mass in unit volume, is shown in Fig. 7.7. At axial position near downstream of nozzle, droplets are highly concentrated in the center, indicating a red colored area. When the axial position increases, droplets disperse in radial direction and a uniform droplet radial distribution is formed gradually, indicating a uniform light-blue colored area, which begins to show up at half length of the feeder tube. Thus, in order to achieve a good droplet mix, the feeder tube length should not less than $250 \mathrm{~mm}$.

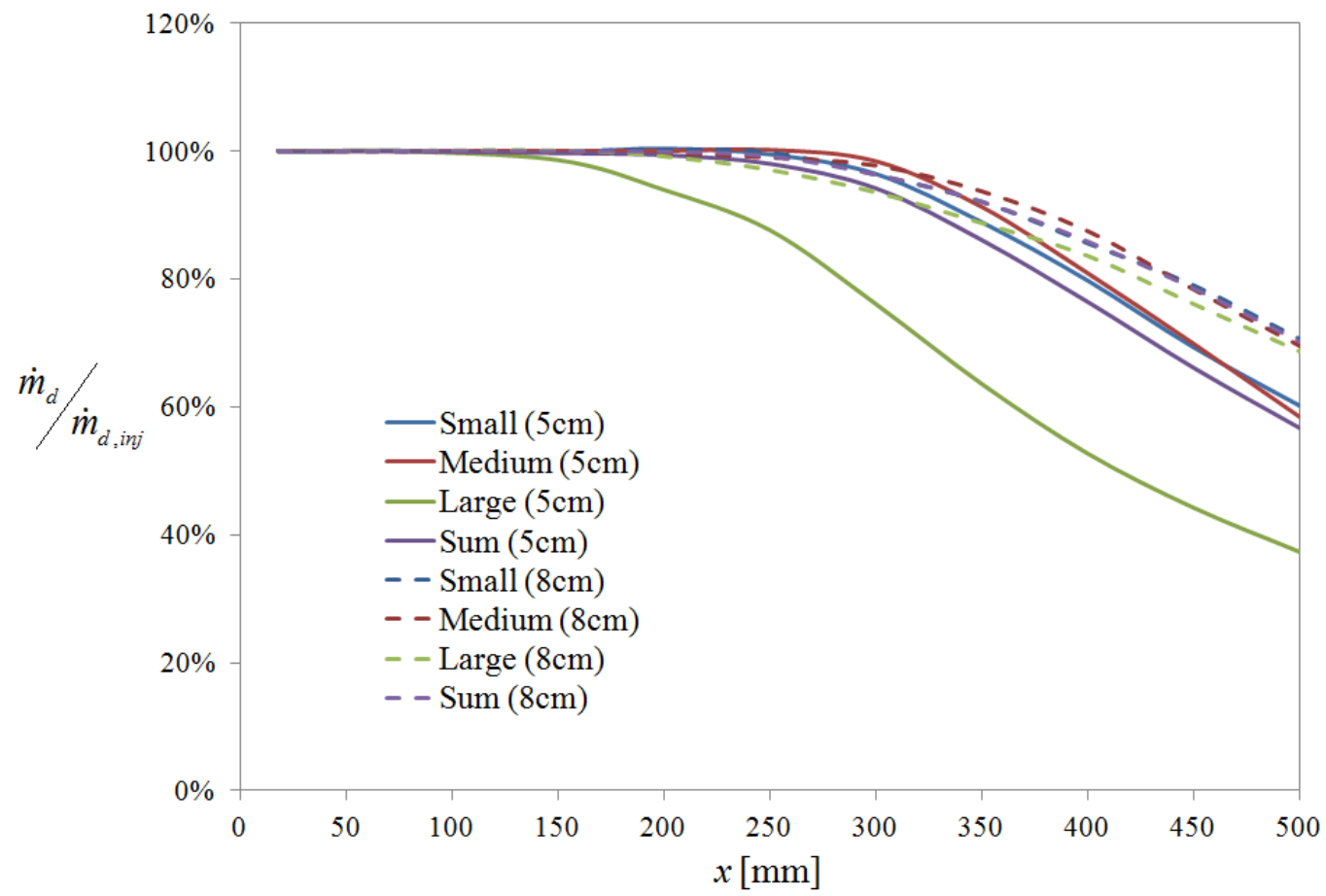

Fig. 7.8 Normalized droplet flow rate as a function of feeder tube axial position

Furthermore, the feeder tube length should be checked with droplet loss rate (DLR) defined at a given axial position of a feeder tube as 


$$
D L R=1-\frac{\dot{m}_{d}}{\dot{m}_{d, i n j}}
$$

In Fig. 7.8, normalized droplet flow rate is employed to show droplet loss rate. Herein, $100 \%$ normalized droplet flow rate indicates a zero droplet loss rate based Eq. 711. It can be seen from this figure that all normalized droplet flow rates are similarly decreasing along axial position. Taking large droplets in 5-cm tube as example (green solid line), the droplet loss can be separated into 3 portions. In the first region $(x<120$ $\mathrm{mm}$ ), droplets do not impact the side wall and the turbulence is not yet fully developed, so the droplet loss is zero. In the second region $(120 \mathrm{~mm}<x<250 \mathrm{~mm})$, droplets begin hitting the wall and droplets are lost, so the droplet flow rate decreases with a slow slope. In the third region $(x>250 \mathrm{~mm})$, the loss of droplets becomes more rapid due to increased turbulence and drop concentration near the wall. Other droplet sizes have the same three-region trend, but the axial ranges are shifted. For example, the small and medium droplets do not have any significant impact until $\mathrm{x}=150 \mathrm{~mm}$. When comparing the two different feeder tube sizes, the $8-\mathrm{cm}$ case has a more reduced droplet rate indicating overall better performance. If an objective is to restrict droplet loss rate to within $10 \%$ while maximizing radial uniformity, the $8-\mathrm{cm}$ feeder tube at the axial position of $300 \mathrm{~mm}$ provides good performance, where less than $5 \%$ of the droplets are lost and where the radial uniformity is checked by contours of droplet mass concentration (Fig. 7.7). Thus, the feeder tube with a diameter of $8 \mathrm{~cm}$ and a length of $300 \mathrm{~mm}$ is a good candidate for future investigation when integrating with the piston geometry. 


\section{Chapter 7 References}

[1] Greenblatt JB, Succar S, Denkenberger DC, Williams RH, Socolow RH. "Baseload Wind Energy: Modeling the Competition Between Gas Turbines and Compressed Air Energy Storage for Supplemental Generation". Energy Policy 2007; 35: 1474-1492.

[2] Li PY, Loth E, Simon TW, Van de Ven JD, Crane SE. "Compressed Air Energy Storage for Offshore Wind Turbines". 2011 International Fluid Power Exhibition (IFPE), Las Vegas, NV, March, 2011.

[3] Qin C, Loth E. "Liquid Piston Compression with Droplet Heat Transfer", Applied Energy 2014; 114: 539-550.

[4] Qin C, Loth E, Li P, Simon T, Van de Ven J. "Spray-Cooling Concept for Wind-Based Compressed Air Energy Storage". Journal of Renewable and Sustainable Energy (Submitted).

[5] Launder BE, Spalding DB, "Lectures in Mathematical Models of Turbulence", London, England: Academic Press; 1972.

[6] Loth E. "Quasi-steady shape and drag of deformable bubbles and drops", International Journal of Multiphase Flow 2008; 34(6): 523-546.

[7] Morsi SA, Alexander AJ. "An Investigation of Particle Trajectories in Two-Phase Flow Systems", Journal of Fluid Mechanics 1972; 55(2): 193-208.

[8] Bocksell TL, Loth E. "Random Walk Models for Particle Diffusion in Free-Shear Flows", AIAA Journal 2001; 39(6): 1086-1096.

[9] Santolaya JL, Aisa LA, Calvo E, Garcia I, Garcia JA. "Analysis by Droplet Size Classes of the Liquid Flow Structure in a Pressure Swirl Hollow Cone Spray", Chemical Engineering and Processing, 2010; 49: 125-131.

[10] Wang XF, Lefebvre AH. "Mean Drop Sizes from Pressure-Swirl Nozzles", Journal of Propulsion 1987; 3(1): 11-18. 


\section{Chapter 8 Summary and Recommendations}

In Chapter 2, it shows the potential advantages of combining spray-based heat transfer with a liquid piston concept for off-shore wind energy storage as compared to traditional ground-based CAES. The injected drops can create a nearly isothermal compression with high compression efficiency. By varying droplet mass loading and diameter, the total surface area of droplet injected can be controlled. The impact of the spray-based heat transfer was considered for a first-stage compressor which may be relevant to a storage system for a wind-turbine. The methodology was developed based on detailed multiphase physics, which were validated with comparison to a set of spraycooling experiments as discussed in the Appendix. This validation used well-know empirical correlations and included discretized differential equations to characterize the air density, water vapor mass fraction, droplet diameter, droplet velocity, air temperature, and droplet temperature.

The quantitative results indicated that a large spray discharge (as measured in terms of the mass loading) provides a more efficient compression cycle. When mass loading is fixed, reducing droplet size allows additional benefits since it allows for a larger total surface for heat transfer. However, the benefits of smaller droplet size tend to be limited to droplet diameters of 20-micron for the current configurations, since this corresponds to conditions where the droplet thermal response time becomes negligible compared to the compression cycle. Two fundamental methods of spray injection were considered: pre-mixed injection upstream of the intake valve and direct injection within the compression chamber. Direct injection yielded superior performance to pre-mixed 
injection because many more droplets were aloft during the critical portion of the compression cycle.

For the baseline condition of 20 micron droplets at a mass loading of unity, direct injection with a compression ratio of ten improved the compression efficiency from about $71 \%$ for the adiabatic conditions to more than $95 \%$ for the spray-cooled condition. With an increase in mass loading to a value of five, the overall efficiency was further increased to $98 \%$. At higher compression ratios, the efficiency for spray-based cooling showed modest reductions. However, two- and three-dimensional flow details coupled with effects of droplet collisions, nozzle and valve characteristics, and multi-stage characteristics should be considered to evaluate the challenges and benefits of this energy storage concept.

The work of Chapter 3 proposes and analyzes a concept for a nearly isothermal multi-stage compressed air energy storage system for wind turbines. In particular, a three-stage $35 \mathrm{MPa}$ storage system with an open accumulator and direct injection spraycooling is designed. The spray-cooling increases efficiency dramatically as compared to an adiabatic compression/expansion system. For example, spray injection with a mass loading of unity and 30 micron droplets yields a compressive efficiency of $89.0 \%$, much larger than the (no-spray) adiabatic efficiency of $26.7 \%$. This high spray-cooling efficiency is based on spray atomization with pressure swirl nozzles (which require no gas injection) to provide the necessary large liquid flow rates and small droplet sizes.

As shown in this chapter, this spray-based cooling concept for compressed-air energy storage would have a small but finite reduction in the wind turbine capacity factor. 
However, this may be offset by a large increase in the Levelization factor (introduced herein as the ratio of average power generated to peak generator rating). This factor is a key performance parameter that can be used to quantify wind energy storage systems and the present analysis shows that spray-enhanced open accumulator air storage can increase the levelization factor of a traditional wind turbine from $35 \%$ to $88 \%$. This increase dramatically reduces the size and capacity of the electrical generator and that of the power transmission lines, which can results in direct cost savings. As a side benefit, the electrical generator is moved from the top of the turbine tower (conventional design) to the tower base (energy storage design). This reduces the mass supported by the tower (which reduces tower structural stiffness requirements) and simplifies generator design and maintenance. While the proposed concept shows many advantages, more detailed analysis, design and experiments are required to determine whether such a system would provide a practical and comprehensive solution to reduce the cost and solve the energy mismatch issues associated with multi-MW offshore wind turbines.

The investigation in Chapter 4 demonstrates a numerical approach to predict droplets and gas flow distributions for a pressure-swirl nozzle, by employing initial conditions just after the liquid sheet break-up and droplet formation. This approach can save both mesh resolution and CPU time by avoiding simulation details of the complicated break-up region. In the present approach, inputs of both continuous and disperse phases (including velocity, flow rate, and turbulence) are introduced at the inflow boundary $(x=9 \mathrm{~mm})$ of an axisymmetric domain. The spherical droplets were simulated by using three discrete diameter bins (small at $7.5 \mu \mathrm{m}$, medium at $25 \mu \mathrm{m}$, and large at $55 \mu \mathrm{m}$ ) to represent the polydisperse size distribution. The time-averaged 
simulation results show good agreement of the axial velocity of both the gas and the droplets with the experimental data in terms of both radial and streamwise spatial distributions. The development of the average spray structure is reasonably predicted, with all droplet sizes showing a smooth radial distribution of mass flux with a peak flux away from the centerline. The trends of increasing radial penetration for the larger droplets relative to the smaller ones was well predicted by the experiments, but there was an over-prediction of the radial peak location for the largest size drops. This is attributed to a lack of collisions accounted for by the simulations, which would tend to increase the flux of larger particles near the centerline as smaller particles are absorbed. Finally, the predicted particle turbulent kinetic energy agrees with theory, yielding that particle velocity fluctuations are governed by their local Stokes numbers and the underlying gas flow turbulence.

In Chapter 5, based on validated theoretical analysis, the mass of large wind turbine tower can be reduced when using hydraulic-electric design while maintaining a consistent safety factor for the tower natural frequency relative to the rated rotor frequency. For instance, half of tower mass can be saved for a 10-MW rated wind turbines. Furthermore, compressed air energy storage system can be integrated to this new design. Employing the inner volume of tower as storage accumulator, there indicates a large energy storage capacity for one day. All of them show benefits of hydraulic-electric off-shore wind turbines, especially for 20-MW wind turbine. Of course, further study is necessary to investigate detailed information about tower mass saving using analysis codes, which can deal with higher modal frequencies. 
In Chapter 6, multi-phase CFD simulations are implemented in an axisymmetric domain to investigate compression in a cylinder using a single nozzle to discharge spray. With an injected mass loading of 1.6 and an initial pressure of one atmosphere, the compression efficiency was found to be as high as $93 \%$ for a one-dimensional simulation. In general, the inclusion of two-dimensional and turbulent flow effects investigated in the axisymmetric simulations yielded similar results to the one-dimensional results, but led to either an increase or a decrease in compressive efficiency depending on the detailed flow physics. One key difference noted in the axisymmetric simulations is the vortex formed in the closed cylinder. This vortex helped keep small aloft droplets for longer periods, which promoted heat transfer. However, small droplets were also found to be strongly affected by turbulent fluctuations and the radial dispersion causing significant droplet losses due to impact at the side wall and the piston surface. This led to a transient mass loading which never reaches the injected mass loading. Additionally, it is shown that the droplet mass distribution is not spatial uniform. For future work, it is suggested that multi-nozzle configurations could be considered with side-wall injection to increase mass loading and reduce. This would require a three-dimensional flow approach, as would the inclusion of intake and exhaust valves, which would allow a better understanding of the full cycle performance.

In Chapter 7, two feeder tubes with different diameter are investigated in CFD simulation. Employing a single pressure swirl nozzle working at maximum experimental liquid flow rate, both of the feeder tube can achieve a unity mass loading (with different gas co-flow rate). In both cases, for gas phase, at the axial position near downstream of the injection, mean velocity and fluctuating velocity is higher in the center. When the 
axial position increases, the radial distribution of both gas mean velocity and its fluctuation trend to become uniform. Thus, in order to get this flat radial distribution, length of the feeder tube cannot be short. For droplets, small and medium droplets perform similarly to each other in the feeder tube. When the axial distance increases, droplets spread in radial direction thanks to turbulence. For large droplets, because of the large momentum, they first hit the side-wall and get lost. Thus, based on droplet dynamics, in order to achieve a good droplet mix, the feeder tube length should not less than $250 \mathrm{~mm}$. Furthermore, droplet loss can be separated into three portions: zero loss, loss caused by hitting walls, and loss caused by turbulent diffusion. Along the axial position, the droplet loss rate increases. It is also found that within same axial distance, turbulence causes more droplet loss than droplet hitting the wall. Finally, considering overall feeder tube performance, the feeder tube with a diameter of $8 \mathrm{~cm}$ and a length of $30 \mathrm{~cm}$ is chosen within acceptable droplet loss rate less than $5 \%$.

Copyright 2014 Chao Qin

All Rights Reserved 